\title{
Laboratory investigation of electrostatic ion waves modified by parallel -ion -velocity shear
}

\author{
Catalin Teodorescu \\ West Virginia University
}

Follow this and additional works at: https://researchrepository.wvu.edu/etd

\section{Recommended Citation}

Teodorescu, Catalin, "Laboratory investigation of electrostatic ion waves modified by parallel -ion -velocity shear" (2003). Graduate Theses, Dissertations, and Problem Reports. 1841.

https://researchrepository.wvu.edu/etd/1841

This Dissertation is protected by copyright and/or related rights. It has been brought to you by the The Research Repository @ WVU with permission from the rights-holder(s). You are free to use this Dissertation in any way that is permitted by the copyright and related rights legislation that applies to your use. For other uses you must obtain permission from the rights-holder(s) directly, unless additional rights are indicated by a Creative Commons license in the record and/ or on the work itself. This Dissertation has been accepted for inclusion in WVU Graduate Theses, Dissertations, and Problem Reports collection by an authorized administrator of The Research Repository @ WVU.

For more information, please contact researchrepository@mail.wvu.edu. 


\title{
Laboratory Investigation of Electrostatic Ion Waves Modified by Parallel-Ion-Velocity Shear
}

\author{
Catalin Teodorescu \\ Dissertation submitted to the Eberly College of Arts and Sciences \\ at West Virginia University in partial fulfillment \\ of the requirements for the degree of \\ Doctor of Philosophy \\ in \\ Physics
}

Mark E. Koepke, Ph.D., Chair

Earl E. Scime, Ph.D.

John E. Littleton, Ph.D.

James Smith, Ph.D.

Gurudas Ganguli, Ph.D.

Department of Physics

Morgantown, West Virginia

2003

Keywords: Velocity Shear, Inverse Ion-Cyclotron Damping, Ion-Cyclotron Wave, Ion Acoustic Wave. 


\author{
ABSTRACT \\ Laboratory Investigation of Electrostatic Ion Waves \\ Modified by Parallel-Ion-Velocity Shear \\ Catalin Teodorescu
}

The objective of this work is the experimental study of the effect of parallel-ionvelocity shear on the destabilization and propagation of electrostatic ion waves. Shear in the magnetic-field-aligned (parallel) ion drift velocity of a single-drifting Maxwellian ion velocity distribution is created in the cylindrical barium plasma column produced in a $\mathrm{Q}$ machine. Standard, emissive, and single-sided Langmuir probes are used to measure plasma parameters and their fluctuations. Direct, noninvasive, measurements of the ion velocity distribution (yielding ion drift velocity and ion temperature) are performed using the laser-induced fluorescence technique. The use of laser-induced fluorescence to determine the radial profile of parallel ion drift velocity permits the measurement of the ion parallel-velocity shear without the weighting by the ion density contributions that are inherent in particle-fluxaveraged measurements. Using an almost isotropic-temperature plasma, multi-harmonic ioncyclotron waves are identified, characterized, and compared with theoretical predictions. Experimental evidence is presented that ion cyclotron damping can become inverted to result in net growth for sufficiently small values of the wavevector components ratio, $k_{/ /} / k_{\perp}$, in the presence of shear in the parallel ion flow. Cases where, for larger values of $k_{/ /} / k_{\perp}$, the damping reduces without changing sign are presented. For other experimental conditions, lowfrequency ion acoustic waves are identified, characterized and compared with theory. Their growth and propagation are measured in anisotropic plasma. Evidence is presented that shear in the parallel ion flow increases the wave frequency, that this increase is responsible for reducing ion Landau damping, and that increasing ion-temperature anisotropy both increases the growth rate and decreases the preferred propagation angle of these ion-acoustic waves. These experimental results serve to benchmark a theoretical model that predicts both shearmodified ion cyclotron and shear-modified ion acoustic waves. In the experiments reported here, the value of parallel-ion-velocity shear ranges from $-0.65 \omega_{c i}$ to $0.24 \omega_{c i}$, the value of ion-temperature anisotropy ranges from 1.2 to 2.5 , the electron-to-ion (parallel) temperature ratio ranges from 1.4 to 2.9 , the perpendicular wavevector component $k_{\perp} \rho_{i}$ ranges from 0.03 
to 1.25 , the parallel wavevector component $k_{||} \rho_{i}$ ranges from $6 \times 10^{-3}$ to $2 \times 10^{-1}$ and the growth rate ranges from $2.2 \times 10^{-3} \omega_{c i}$ to $3.9 \times 10^{-3} \omega_{c i}$. The results of this work provide experimental support for the inhomogeneous-parallel-ion-flow-model interpretation of multi-harmonic electrostatic ion -cyclotron waves and ion-acoustic waves observed in the auroral region. The results also broaden fundamental plasma physics concepts such as ion-cyclotron damping to include the possibility of inverse ion-cyclotron damping. 


\section{Acknowledgements}

It is my pleasure to acknowledge the constant and fruitful support I received during my research activity from Prof. Mark Koepke. I am indebted to him for all aspects of my work in plasma physics and nonlinear dynamics. I also benefited from numerous stimulating discussions with Prof. Earl Scime, Dr. Valeriy Gavrishchaka and Dr. Gurudas Ganguli, who helped me understand theoretical aspects of the work presented in this dissertation. The use of and help with the computer code that solves the analytical dispersion relation from Dr. Gavrishchaka and Dr. Ganguli is gratefully acknowledged.

The initial stage of the work was also made possible through the help I received from Dr. Michael Zintl and Dr. Guiding Wang. I deeply appreciate fellow graduate student Eric Reynolds for his the direct help in setting-up experiments, sharing some of his data and using some of his computer programs for data analysis. The stimulating discussions with Dr. John Kline and Robert Spangler are also acknowledged.

I thank Prof. Joel Fajans and Dr. Eli Sarid for the support I received during my work at University of California, Berkeley. The experience I gained working with Prof. Fajans' group has broadened my knowledge of non-neutral plasmas.

The encouragement of my family is the foundation of success in all my endeavors. 


\section{Contents}

Abstract......................................................................................

Acknowledgements.................................................................

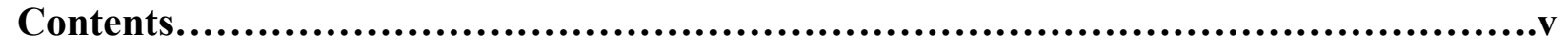

List of Tables........................................................................vii

List of Figures................................................................... viiii

Chapter I. Introduction......................................................1

I.A Inhomogeneous magnetic-field-aligned (parallel) ion flow..................................

I.B Basic plasma physics of electrostatic ion waves ...............................................

I.C Electrostatic ion cyclotron and ion acoustic waves in the presence of parallel ion

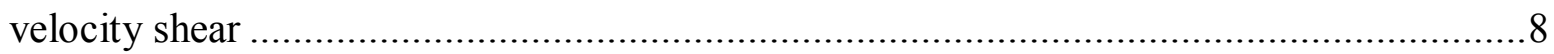

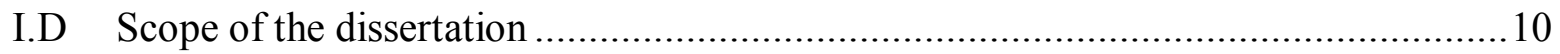

Chapter II. Theory..........................................................12

II.A Electrostatic ion cyclotron waves modified by shear in the parallel-ion-velocity ......12

II.B Electrostatic ion acoustic waves modified by shear in the parallel-ion-velocity ........25

Chapter III. Experimental device...............................................31

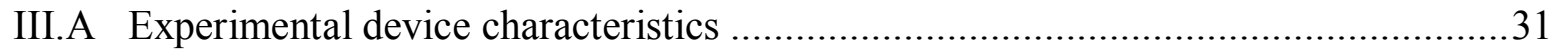

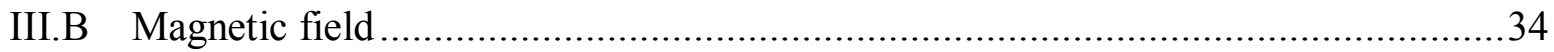

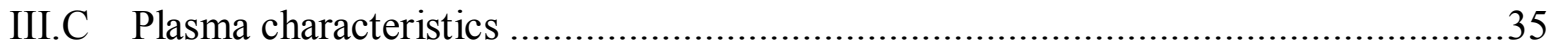

Chapter IV. Diagnostics..............................................................36

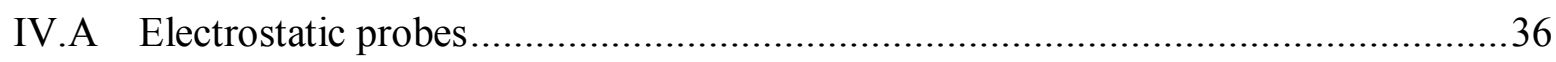

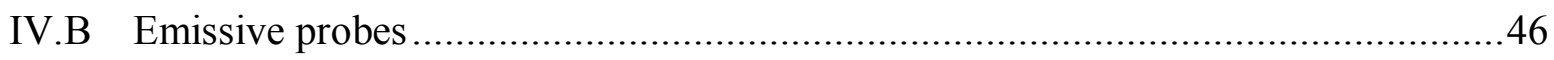

IV.C Laser induced fluorescence diagnostics .....................................................47

IV.C.1 Laser induced fluorescence apparatus ......................................................49

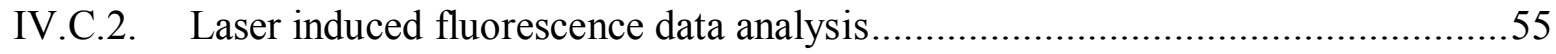

Chapter V. Experimental results .............................................63

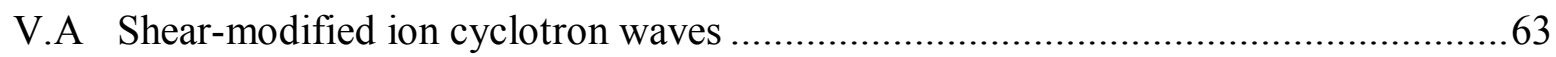

V.A.1 Control of the ion parallel drift velocity radial profile ........................................63

V.A.2Characteristics of the shear-modified multi-harmonic ion cyclotron instability........65

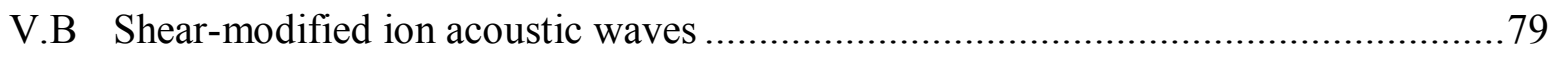

V.B.1 Control of the ion-parallel-velocity drift radial profile...................................... 79

V.B.2Characteristics of the shear-modified ion acoustic instability ............................... 83 


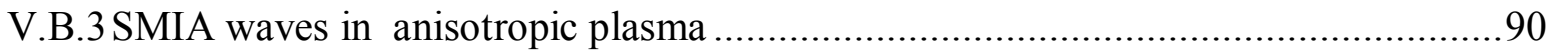

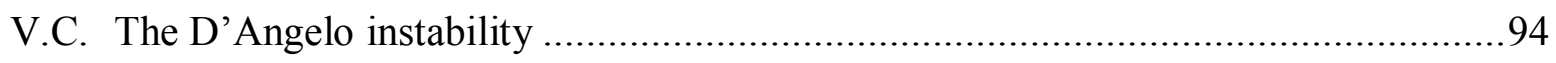

Chapter VI. Discussions and Conclusions................................................97

References........................................................................108

Tables...........................................................................115

Figures................................................................................120

Appendix 1..........................................................................196

Appendix 2 ......................................................................198

Appendix 3............................................................................200

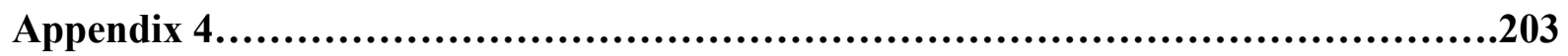

Appendix 5......................................................................206

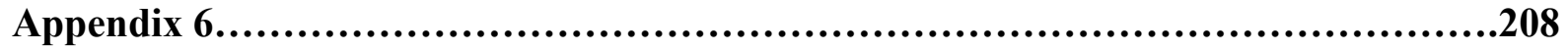




\section{List of Tables}

Table 1 WVU Q machine barium-plasma parameters.

Table 2 Device settings used for controlling the parallel ion drift velocity radial profile in the Single Ionizer Single Electrode configuration of the Q machine.

Table 3 Values of the parallel-velocity shear and of the multi-harmonic EIC wave parameters in the case of inverse ion cyclotron damping. Here, $\mathrm{n}$ is the harmonic number.

Table 4 Device settings used for controlling the parallel ion drift velocity radial profile in the Double Ionizer No Electrode configuration of the Q machine. Magnetic field direction is southwards. Shear magnitude increases with increasing $\left|V_{N}-V_{S}\right|$. Shear sign switches from positive when $\mathrm{V}_{\mathrm{N}}<\mathrm{V}_{\mathrm{S}}$ to negative when $\mathrm{V}_{\mathrm{N}}>\mathrm{V}_{\mathrm{S}}$, if both atomic ovens are on.

Table 5 Control of the SMIA waves propagation direction adjusting the bias voltages applied to the ionizers.

Table 6 Control of the SMIA waves propagation direction adjusting the emitted power at the ionizers.

Table 7 Plasma and wave parameters associated with the graphs in Fig. 66 and Fig. 68. 


\section{List of Figures}

Fig. 1 WVU Q machine (not-to-scale schematic) in the Single Ionizer Single Electrode configuration: here, neither ions nor electrons are produced by the south plasma source. Magnetic field direction can be reversed. $z=0$ is at the ionizer from which the magnetic field points out.

Fig. 2 WVU Q machine magnetic field strength along the Q machine center axis.

Fig. 3 Typical current-voltage characteristic (measured at $z=69 \mathrm{~cm}$ from the north ionizer) of a Langmuir probe immersed in barium plasma created in the WVU Q machine. Here, $B=0.14 \mathrm{~T}, n=3.2 \times 10^{9} \mathrm{~cm}^{-3}, T_{e}=0.29 \mathrm{eV}$ and $T_{i}=0.23 \mathrm{eV}$. Ionizers are both biased at $-1 \mathrm{~V}$.

Fig. 4 Barium plasma density radial profile at $z=69 \mathrm{~cm}$ from the north ionizer. $(B=0.14 \mathrm{~T}$ and $V_{\text {probe }}=-7 \mathrm{~V}$ ). Radial position $r=0$ corresponds to the cylindrical axis of the vacuum chamber.

Fig. 5 Plasma density fluctuation in the presence of the D'Angelo instability (measured at $z=69 \mathrm{~cm}$ from the north ionizer) when $B=0.11 \mathrm{~T}, V_{\text {probe }}=-5 \mathrm{~V}$ and $n_{0}=2.6 \times 10^{9} \mathrm{~cm}^{-3}$.

Fig. 6 (a) Two-tip probe (double Langmuir probe) and (b) two-tip probe rotation with respect to the magnetic field lines.

Fig. 7 Dependence of relative phase difference between probe-array tips on the orientation of the probe array with respect to the magnetic field. ( $B=0.2 \mathrm{~T}$ southward and $z=46 \mathrm{~cm}$ from the north ionzier). The solid black line is the best sinusoidal fit yielding $k_{z}=$ $-0.038 \pm 0.005 \mathrm{~cm}^{-1}$ and $k_{y}=-3.221 \pm 0.041 \mathrm{~cm}^{-1}$; the dashed line is the same sinusoid with $k_{z}=0$.

Fig. 8 Perpendicular wavevector component $k_{y}$ spectrum $(B=0.2 \mathrm{~T})$. Probe tip separation is $3.5 \mathrm{~mm}$ yielding $k_{y l}=0.69 \mathrm{~cm}^{-1}$ and $k_{y 2}=0.72 \mathrm{~cm}^{-1}$, respectively, for the two largest components shown.

Fig. 9 SMIA wave relative phase between one probe tip at $x=1.5 \mathrm{~cm}, y=1.5 \mathrm{~cm}, z=138 \mathrm{~cm}$ and another probe tip at $-2<x<2 \mathrm{~cm},-2<y<2 \mathrm{~cm}$ and $z=161 \mathrm{~cm}$. The approximately $180^{\circ}$ phase difference between the wave phase measured in the points located at $x=-1$ $\mathrm{cm}, y=2 \mathrm{~cm}$ and $x=2 \mathrm{~cm}, y=-2 \mathrm{~cm}$, respectively, indicates an azimuthal mode number $m=1$. 
Fig. 10 Parallel electron drift radial profile inferred from single-sided probe measurements. $\left(B=0.15 \mathrm{~T}, z=46 \mathrm{~cm}, v_{\mathrm{ti}}=520 \mathrm{~m} / \mathrm{s}\right)$. Electron drift is parallel to $B$ here. Here, $\zeta=\left|v_{\mathrm{de}} / v_{\mathrm{te}}\right|$.

Fig. 11 Schematic diagram of LIF apparatus corresponding to the perpendicular-velocity measurement.

Fig. 12 Schematic of the LIF parallel injection optics mounted on the vacuum chamber (not to scale). Angle $\alpha$ is adjustable by rotating the goniometer that holds the fiber-optic cable.

Fig. 13 Perpendicular injection optics and collection optics mounted on the vacuum chamber.

Fig. 14 Grotrian diagram for BaII transitions relevant to the WVU Q plasma machine LIF. Under each energy-level diagram are shown the polarizations of the respective transitions: $\pi\left(\Delta m_{J}=0\right)$ or $\sigma\left(\Delta m_{J}= \pm 1\right)$; the corresponding spectral intensity (upper indices); the corresponding Lande factors $g_{L}$ (lower indices). Fluorescence transitions are represented in (a); optical pumping is achieved through the transitions shown in (b).

Fig. 15 Laser-induced fluorescence spectrum of barium ions observed when using the parallel injection configuration for the LIF optics.

Fig. 16 Laser-induced fluorescence spectrum of barium ions observed when using the perpendicular injection configuration for the LIF optics.

Fig. 17 Parallel LIF-measured plasma temperature as a function of injection angle $\alpha$.

Fig. 18 Fluorescence spectrum ( $\pi$ lines) of cooled-barium plasma using parallel-injection LIF. Here, $T_{i z}=0.034 \mathrm{eV}$.

Fig. 19 (a) Parallel-LIF spectrum (dots) and the best fitted lineshape; (b) the corresponding single drifting Maxwellian distribution obtained from the fitted lineshape.

Fig. 20 (a) Perpendicular-LIF spectrum (dots) and the best fitted lineshape ; (b) the corresponding single drifting-Maxwellian distribution obtained from the fitted lineshape.

Fig. 21 (a) Parallel LIF-measured barium spectrum (dots) and the best fitted lineshape; (b)the corresponding two drifting-Maxwellian distributions obtained from the fitted lineshape and their sum (line).

Fig. 22 (a) Parallel-LIF-measured barium spectrum (dots) and the best fitted lineshape in 
the Q machine set up in SISE configuration. (b) The corresponding single drifting Maxwellian velocity distribution.

Fig. 23 Inhomogeneous parallel ion drift velocity radial profile obtained using the annular electrode biased at $V_{A}=35 \mathrm{~V}$ and the termination plate biased at $V_{T}=-5 \mathrm{~V}$.

Fig. 24 Laser-induced fluorescence measurement of the radial profile of barium-ion parallel drift velocity, representative of (a) negligible shear using either annular or button electrode (here, button with $V_{0}=20 \mathrm{~V}, V_{\mathrm{T}}=0,1.4 \mathrm{kG}$ ), (b) nonzero shear using annulus $\left(V_{0}=35 \mathrm{~V}, V_{\mathrm{T}}=0,2 \mathrm{kG}\right)$, and (c) nonzero shear using button $\left(V_{0}=35 \mathrm{~V}, V_{\mathrm{T}}=0,2 \mathrm{kG}\right)$. The long error bar at the right of (a) represents the ion thermal speed for all data (i.e., $T_{\mathrm{i}}$ is uniform).

Fig. 25 Parallel-ion-drift-velocity shear as a function of radial position associated with velocity profiles (a) and (b) in Fig. 24. Multi-point average value of shear is shown by the two black lines.

Fig. 26 (a) LIF-measured barium spectrum spectrum (dots) and the best fitted lineshape.

(b)The corresponding two drifting-Maxwellian ion velocity.

Fig. 27 (a) Parallel ion drift velocity radial profiles of the direct (parallel) population and (b) of the counter-streaming (anti-parallel) population when two Maxwellian populations are present. Parallel-propagating population is 6 times denser than the anti-parallel-propagating population.

Fig. 28 Plasma density radial profile in the SISE configuration of the Q machine, inferred from ion saturation current measurements.

Fig. 29 Plasma potential radial profile in the SISE configuration. A biased $\left(V_{A}=35 \mathrm{~V}\right)$ annular electrode ( $3.6 \mathrm{~cm}$ outer diameter, $1.75 \mathrm{~cm}$ inner diameter) is located on the cylindrical axis with the surface normal parallel to the magnetic field.

Fig. 30 Magnetic field strength dependence of the multi-harmonic ion cyclotron waves in the presence of inverse ion cyclotron damping. Straight lines are harmonics of the ion gyrofrequency.

Fig. 31 Spectra of ion saturation current fluctuations collected by (a) the annular electrode (negligible-shear case, $V_{0}=15 \mathrm{~V}, V_{\mathrm{T}}=0,2 \mathrm{kG}$ ), (bi) the annular electrode (nonzero-shear case, same as Fig. 24b), (bii) the Langmuir probe (same as previous case (bi)), and (c) the button electrode (nonzero-shear case, same as Fig. 24c).

Fig. 32 Probe-tip phase difference as a function of probe orientation and the best fitted 
sinusoid (black) yielding $k_{y}=-2.120 \mathrm{~cm}^{-1}, k_{z}=0.024 \mathrm{~cm}^{-1}$. The red dashed line is a sinusoid with $k_{y}=-2.080 \mathrm{~cm}^{-1}, k_{z}=0.019 \mathrm{~cm}^{-1}$, the blue dashed one has $k_{y}=-2.160 \mathrm{~cm}^{-1}$, $k_{z}=0.029 \mathrm{~cm}^{-}$, representing the mean value plus/minus the standard deviation.

Fig. 33 The circle method applied to data shown in Fig. 32. Wavevector components are $k_{z}=(0.024 \pm 0.003) \mathrm{cm}^{-1}$ and $k_{y}=(-2.120 \pm 0.020) \mathrm{cm}^{-1}$.

Fig. 34 Intersection of gyroframe-SMIC-wave parallel phase velocity with the parallel-ion -velocity distribution. Dots indicate the local values of the ion parallel drift velocity. The $x$ spacing between the velocity distributions is $0.2 \mathrm{~cm}$.

Fig. 35 Parallel electron drift velocity radial profile in the presence of paralell-velocity shear and multi-harmonic SMIC waves. No structure in the parallel velocity profile is observed to correspond to the radial position of the electrode. Here, $v_{t i}=544 \mathrm{~m} / \mathrm{s}$.

Fig. 36 SMIC waves regimes for fixed shear $d v / d x=0.14 \omega_{c i}$. The three points represent experimental data points. The straight line is the locus of points for which the ion cyclotron damping factor is equal to zero.

Fig. 37 Multi-harmonic SMIC waves fundamental frequency dependency on shear magnitude.

Fig. 38 Multi-harmonic SMIC waves normalized frequency as a function of harmonic number (filled circles). Linear-theory-predicted wave frequency (open circles) for shear-driven multi-harmonic EIC waves (from Gavrishchaka et al., [2000]).

Fig. 39 Multi-harmonic SMIC waves waveform (thick line) in the presence of reduced ion cyclotron damping factor $(=0.38)$ and single-feature ion cyclotron wave waveform (thin line) when the ion cyclotron damping is equal to unity.

Fig. 40 (a) Space-reported measurements of parallel electric field time-domain fluctuations associated with the presence of multi-harmonic electrostatic ion cyclotron waves. [Ergun et al., 1998]. (b) Computer simulations, based on the model of Gavrishchaka et al., [2000], of the parallel electric field time-fluctuations associated with SMIC waves.

Fig. 41 SMIC waves $k_{z}$ spectrum. The double probe is oriented antiparallel to $B$, thus $k_{z}=-0.026 \mathrm{~cm}^{-1}$.

Fig. 42 SMIC waves $k_{y}$ spectrum with the double probe oriented at $90^{\circ}$ with respect to $B$, thus $k_{y}=-2.21 \mathrm{~cm}^{-1}$ 
Fig. 43 SMIC waves amplitude radial profile (squares, right scale) peaks within the shear layer. Measurements are based on the ion saturation current fluctuations recorded by a Langmuir probe. Circles represent the values of the parallel ion drift velocity when using the button electrode (open circles) and the annular electrode (filled circles), respectively.

Fig. 44 Radial profile of the parallel-LIF barium spectrum;(a) both barium ovens on or (b) only the north oven on. Height is proportional to the ion-metastable-state relative density. The signal is double-peaked due to the large Zeeman split. The frequency shift of the signal around $x=0.4 \mathrm{~cm}$ indicates shear in the parallel-ion-drift velocity. The hollow cathode lamp signal has a wavelength $\lambda_{0}=585.5302 \mathrm{~nm}$, indicating small ion drift at $0.4<x<3 \mathrm{~cm}$ and large ion drift at $-2<x<0.4 \mathrm{~cm}$.

Fig. 45 (a) Sample of parallel LIF fitting and (b) the corresponding best fitted singleMaxwellian ion distribution after the Zeeman-split removal.

Fig. 46 Typical parallel ion drift velocity radial profile when SMIA waves are destabilized.

Fig. 47 Parallel electron drift velocity radial profile in the presence of SMIA waves $\left(v_{t i}=480\right.$ $\mathrm{m} / \mathrm{s})$.

Fig. 48 (a)Current-voltage characteristic of plasma in the presence of SMIA waves; (b)current derivative (line) and the best Maxwellian fit (dots).

Fig. 49 (a) Plasma density radial profile inferred from ion saturation current measurements (b) Ion metastable states density radial profile inferred from LIF measurements.

Fig. 50 Plasma-potential radial profile (lower curve, left scale) and the corresponding radial electric field (upper curve, right scale) in the DINE configuration.

Fig 51 SMIA wave spectrum in the lab frame: wave propagating parallel to the ion flow (a); wave propagating anti-parallel to the ion flow (b). Here, $f_{\mathrm{ci}}=13 \mathrm{kHz}$. Doppler shifts are indicated in the text.

Fig. 52 SMIA waves frequency dependency on magnetic field strength.

Fig. 53 Probe-tip phase difference as a function of probe orientation angle. The fitted sinusoid yields $k_{z}=-0.507 \mathrm{~cm}^{-1}$ and $k_{y}=-0.331 \mathrm{~cm}^{-1}$.

Fig. 54 SMIA waves $k_{y}$ wavevector spectrum with the two-tip probe oriented at $90^{\circ}$ with respect to $B . k_{y}=0.09 \pm 0.02 \mathrm{~cm}^{-1}$ corresponds to the maximum amplitude feature.

Fig. 55 Comparison between the measured and the predicted SMIA wave azimuthal wavevector for two cases of propagation mode number $m$. 
Fig. 56 (a) LIF-measured ion parallel-velocity distribution (circles) and the ion sound speed, measured parallel wave phase velocity and calculated wave phase velocity shift.

(b) Derivative of solid line in Fig.56(a). The derivative is assumed proportional to $\gamma_{i L d}$.

Fig. 57 Comparison between parallel (filled circles) and perpendicular (open circles) ion drift velocity profile (left scale), parallel electron drift velocity (line, right scale) in the presence of SMIA waves.

Fig. 58 Comparison between parallel (filled circles) and perpendicular (open circles) ion drift velocity shear in the presence of SMIA waves based on the profiles shown in Fig.57. Measurements are taken at $z=46$ from the north ionizer. Here, $V_{S}=V_{N}=0$ (magnetic field $B=0.13 \mathrm{~T}$ is southward).

Fig. 59 Parallel ion velocity drift radial profile (upper curve, left scale) for positive shear (lower curve, right scale) and no waves destabilized. Here $V_{N}=V_{S}=0, B=0.13 \mathrm{~T}$.

Fig. 60 Correlation between shear, SMIA waves growth rate (filled symbols, left scale) and SMIA wave frequency (open symbols, right scale). Symbols are associated with the values of $u=0.7$ (square), 1.4 (circle) and 1.9 (triangle), respectively. Normalization constant is the ion cyclotron frequency $\omega_{\mathrm{ci}}$.

Fig. 61 (a) Wave amplitude temporal growth after applying the stepped ionizer bias (up). Wave amplitude and the pulse-removing fitting line (down).

Fig. 61 (b) Pulse-extracted wave amplitude (up) and the least square fit of an exponential through the peaks (symbols) of the envelope (down).

Fig. 62 Control over SMIA waves propagation direction using the ionizers' relative bias.

Fig. 63 Control over SMIA waves propagation direction using the ionizers' emitting power.

Fig. 64 LIF measured parallel ion temperature (filled circles) and perpendicular ion temperature (open circles) at the axial position $z=46 \mathrm{~cm}$.

Fig. 65 Experimentally determined SMIA waves propagation angle in anisotropic ion-temperature plasma (filled circles)and optimum-growth predicted propagation angle in isotropic plasma (open circles) as functions of shear magnitude. Filled squares and open squares are the measured and calculated values of $\sigma$. Parameter values are listed in Table 7. 
Fig. 66 SMIA waves growth rate (filled triangle, diamond and square, respectively) and frequency dependency (filled circles) on ion temperature anisotropy. Theoreticallypredicted optimum growth rates (open triangle, diamond and square) in isotropic plasma. Shear values are $-0.45 \omega_{\mathrm{ci}}$ (triangle), $-0.54 \omega_{\mathrm{ci}}$ (diamond) and $-0.62 \omega_{\mathrm{ci}}$ (square), respectively.

Fig. 67 (a) Parallel (filled circles) and perpendicular (open circles) LIF spectra and their corresponding fitted Maxwellian velocity distributions (parallel distribution solid line; perpendicular distribution - dashed line). (b) Derivatives of parallel (solid line) and perpendicular (dashed line) velocity distributions and the measured wave phase velocities, respectively.

Fig. 68 SMIA waves propagation angle in anisotropic ion-temperature plasma.

Fig. 69 Comparison between experimentally-measured SMIA growth rate (circles) in anisotropic barium-plasma and theory predicted (squares) growth rates for analogous plasma parameters.

Fig. 70 Experimentally determined SMIA waves growth rate (filled symbols) in anisotropic ion-temperature barium plasma for $u=0.7$ (triangle), 1.4 (diamond), 1.9 (square) and theoretically predicted values (open symbols) in isotropic barium plasma for identical propagation angles.

Fig. 71 (a) Parallel ion drift velocity radial profile and (b) the shear-destabilized D'Angelo instability amplitude radial profile (inferred from ion saturation current fluctuations on a Langmuir probe). Here $B=0.11 \mathrm{~T}$ (southward), $V_{N}=-4 \mathrm{~V}$, and $V_{S}=10 \mathrm{~V}$.

Fig. 72 D'Angelo instability normalized spectrum.

Fig. 73 D'Angelo instability relative phase difference between probe tips as a function of probe orientation. The fitted sinusoid yields $k_{z}=-0.07 \mathrm{~cm}^{-1}$ and $k_{y}=-0.75 \mathrm{~cm}^{-1}$.

Fig. 74 Parallel electron drift velocity radial profile in the presence D'Angelo instability.

Fig. 75 Low-frequency waves regimes in the presence of parallel-velocity shear: first and third quadrants - D'Angelo instability, second and fourth quadrants - SMIA waves. Circles represent experimental data. 


\section{Chapter I. Introduction}

In this chapter, the definition of ion parallel-velocity shear and some ways of producing it in a laboratory plasma, as previously cited in the literature, are presented. The concept of electrostatic ion-cyclotron and ion-acoustic instabilities modified by shear in the magnetic-field-aligned (parallel) ion flow in magnetized plasma is introduced. The scope of the present laboratory investigation is presented to convey the specific accomplishments of the dissertation.

\section{I.A Inhomogeneous magnetic-field-aligned (parallel) ion flow}

The charged particles (ions, electrons) that form a weakly coupled, quasineutral, plasma have individual kinetic energy that greatly exceeds the individual potential energy. The electric potential created by each charged particle is shielded from the majority of the other charged particles by compensation provided by neighboring charges. The motion of the particles (and thus the plasma evolution) is studied statistically (because of their large number) using the particle distribution function $f(t, \boldsymbol{x}, \boldsymbol{v})$, a statistical quantity that is the particle-number density in six-dimensional coordinate-velocity phase space. The evolution of the distribution function in phase space is described by the Boltzmann equation

$$
\frac{\partial f}{\partial t}+\mathbf{v} \cdot \frac{\partial f}{\partial \mathbf{x}}+\mathbf{a} \cdot \frac{\partial f}{\partial \mathbf{v}}=\left.\frac{\partial f}{\partial t}\right|_{c}
$$

which is a form of the Klimontovich-Dupree equation in which the correlations between the particles, via collisions, are accounted for, but the correlations between the fields (electric, magnetic) are neglected. In many cases, such as in the experiments described here, the number of collisions per unit time is negligibly small, meaning that only very few plasma particles experience collisions. Therefore, the word "collisionless" can be used for the plasma, justifying the approximation

$$
\left.\frac{\partial f}{\partial t}\right|_{c}=0
$$


The presence of an externally applied magnetic field $\boldsymbol{B}$ to the plasma disrupts the overall isotropy of the plasma. For a uniform magnetic field, the electrons and ions can drift with respect to each other along the magnetic field lines. The motion perpendicular to the magnetic field is predominantly influenced by the Lorentz force, hence the electrons and ions can both gyrate and drift perpendicular to the magnetic field lines. The Boltzmann equation becomes

$$
\frac{\partial f}{\partial t}+\mathbf{v} \cdot \frac{\partial f}{\partial \mathbf{x}}+\frac{q}{m}(\mathbf{E}+\mathbf{v} \times \mathbf{B}) \cdot \frac{\partial f}{\partial \mathbf{v}}=0
$$

in collisionless plasmas, also named the Vlasov equation. In the absence of collisions, the particle-number density remains constant. Practically, this behavior is described by Liouville's theorem.

The measured distribution function is used for finding physical quantities related to the particle-number density. These following macroscopic quantities are defined as the velocity moments of the distribution:

$$
\begin{array}{ll}
\text { density (the zero-order moment): } & n(t, \mathbf{x})=\int f(t, \mathbf{x}, \mathbf{v}) d^{3} v \\
\text { drift velocity (the first-order moment): } & \mathbf{v}_{d}(\mathrm{t}, \mathbf{x})=\frac{1}{\mathrm{n}} \int \mathbf{v} f(t, \mathbf{x}, \mathbf{v}) d^{3} v \\
\text { pressure (the second-order moment): } & \mathbf{P}(t, \mathbf{x})=m \int\left(\mathbf{v}-\mathbf{v}_{\mathrm{d}}\right) \circ\left(\mathbf{v}-\mathbf{v}_{d}\right) f(t, \mathbf{x}, \mathbf{v}) d^{3} v
\end{array}
$$

where "o" denotes the tensor product. The temperature $T$ of the plasma species is defined based on the species distribution function. This statistical scalar quantity comes from the diagonal terms of the pressure tensor, being defined as $T=\frac{m}{3 k_{B} n} \int\left(\mathbf{v}-\mathbf{v}_{\mathrm{d}}\right) \cdot\left(\mathbf{v}-\mathbf{v}_{\mathrm{d}}\right) f d^{3} v$.

Sometimes, the equilibrium velocity distribution function of a collisionless plasma is the Maxwellian velocity distribution, in which case the plasma is in thermal equilibrium. The particle velocities are distributed according to a Gaussian distribution around the average velocity, which is zero for a plasma at rest.

In the case that the plasma is not at rest the drifting-Maxwellian distribution is sometimes applicable, $f(\mathbf{v})=n\left(\frac{m}{2 \pi k_{B} T}\right)^{3 / 2} \exp \left[-\frac{m\left(\mathbf{v}-\mathbf{v}_{d}\right)^{2}}{2 k_{B} T}\right]$. Plasmas at rest have $\boldsymbol{v}_{\boldsymbol{d}}=0$. 
Anisotropic plasmas are described using distributions that take into account the fact that plasma properties are different along different directions. An important case is that of gyrating particles, when the velocity distribution does not depend on the gyrophase. In this case, the distribution depends only on the velocity components parallel to and perpendicular to the magnetic field, respectively. Because these components are independent, the equilibrium distribution can be modeled as the sum of two Maxwellian distributions, the resulting distribution function being called a bi-Maxwellian distribution.

Sometimes, for analysis of plasma stability or approximate characterization of observations, the drift velocity is idealized to be homogeneous. Inhomogeneity in the drift velocity can arise if the flow is sheared. Previous dissertations from the WVU Plasma Physics Laboratory addressed aspects of shear in the perpendicular component of electron and ion velocities $d v_{E x B} / d x$ [Amatucci, 1994; Gavrishchaka, 1996; Carroll, 1997]. In this dissertation, aspects of shear in the parallel component of ion velocity $d v_{d i} / d x$ are addressed. Here, $z$ is the direction of the magnetic field, $x$ is the inhomogeneity direction and $y$ is the third coordinate of the Cartesian system. In the cylindrical geometry, the coordinates are $z, r$ and $\theta$, respectively.

For drifting-Maxwellian populations, each population is characterized by its own velocity shear, which can be positive, negative or zero. When two populations occupy the same physical space a flux-averaged description of multiple populations introduces a density weighting of each population's contribution to the average. Shear in the flux-averaged velocity is, in general, different from the shear in an individual population velocity. This difference can be especially dramatic in the case of counter-streaming populations.

In the fluid model describing the inhomogeneous magnetized plasma stability, the relevant equations are the continuity equation

$$
\frac{\partial n}{\partial t}+\nabla \cdot(n \mathbf{v})=0
$$

and the equation of motion

$$
m n \frac{d \mathbf{v}}{d t}=-\nabla p+e n \mathbf{v} \times \mathbf{B}_{0}
$$

where $n$ is particle-number density, $\boldsymbol{v}$ is the fluid-element velocity, $m$ is the particle mass, $p$ is the particle pressure, $e$ is the electric charge unit and $\boldsymbol{B}_{\boldsymbol{0}}$ is the magnetic field strength. No externally applied electric field is included in this equation. 
Sheared parallel flow creates a diamagnetic drift and introduces nonzero off-diagonal elements in the pressure tensor. For the case of shear in the parallel ion velocity, using a kinetic treatment, Smith and von Goeler [1968] obtained the following expression:

$$
p_{0 i}=n_{0} k_{B} T_{i}\left(\begin{array}{lll}
1 & 0 & 0 \\
0 & 1 & \gamma \\
0 & \gamma & 1+\gamma^{2}
\end{array}\right)
$$

where $\gamma=\frac{d v_{d i}}{d x}\left(\omega_{c i}\right)^{-1}, \omega_{c i}$ is ion gyrofrequency, $n_{0}$ is plasma density, $k_{B}$ is Boltzmann's constant and $T_{\mathrm{i}}$ is ion temperature.

Mikhailovskii [1992] used a hydrodynamic model to obtain the equilibrium values of the off-diagonal terms of the pressure tensor in an isotropic, collisionless plasma with no density gradient.

$$
p_{z y}=p_{y z}=\frac{n_{0} k_{B} T_{i}}{\omega_{c i}} \frac{d v_{d i}}{d x}
$$

In the case when small perturbations to the equilibrium values of the parallel ion velocity are allowed, $v_{1 d i} \neq 0$, the off-diagonal terms of the perturbed-pressure tensor become

$$
\begin{aligned}
& p_{1 x z}=-i \frac{p_{0}}{\omega_{c i}} k_{y} v_{1 d i}=p_{1 z x} \\
& p_{1 y z}=\frac{p_{0}}{\omega_{c i}}\left[\frac{\partial v_{1 d i}}{\partial x}+\frac{p_{1 i}}{p_{0}} \frac{d v_{d i}}{d x}\right]=p_{1 z y}
\end{aligned}
$$

Here, $p_{0}=n_{0} k_{B} T_{i}$ and $p_{1 i}=n_{1} k_{B} T_{i}$ where $n_{1}$ is the perturbed part of the density $n_{0}$. Mikhailovskii's treatment can be applied to plasmas with very low or close-to-unity values of $\beta=\left(8 \pi n_{0} T\right) / B_{0}^{2}$ (here, $B_{0}$ is the strength of the magnetic field).

The shear-induced diamagnetic drift velocity can be determined from (4) and (5) with the knowledge that $v_{d i}(x)=\left.v_{d i}\right|_{x=0}+\left(d v_{d i} / d x\right) x$, so that

$$
\mathbf{v}_{D}=\frac{v_{\perp t i}^{2}}{\omega_{c i} v_{0 z}} \frac{d v_{d i}}{d x} \hat{\mathbf{y}}
$$

where $v_{\perp t i}{ }^{2}=\left(k_{B} T_{\perp i} / m_{i}\right)$ is the square of the ion thermal speed perpendicular component. 
The case of nonzero shear-induced diamagnetic drift velocity is described in this dissertation. More precisely, the effect of shear in the parallel ion velocity on the destabilization and propagation of ion cyclotron and ion acoustic waves is investigated.

\section{I.A.1 Inhomogeneous parallel ion flow in laboratory devices: production and measurements}

The effort to produce controllable inhomogeneous parallel ion flow in laboratory devices dates back to D'Angelo and von Goeler [1966] who used a set of two blocking electrodes in a so called "ring and disk" configuration in a double-ended Q-machine. "Double ended" refers to the use of plasma sources at both ends of the plasma column. Plasma is produced on hot $(2000 \mathrm{~K})$ circular, refractory, metal plates by thermionic emission of electrons and by contact-ionization of alkali metals. A solenoidal magnetic field maps the plasma from one plasma source to the other, forming the column. The blocking electrodes, the normal of which is aligned with the cylindrical axis, are separated by a large fraction of the plasma column. The ring electrode admits only plasma at vector radii less than its inner diameter while the disk electrode admits plasma at radii greater than its outer diameter. Since the inner and outer diameters mentioned here are identical, coaxial plasmas are formed, each originating from a different source and making contact at a cylindrical-shell interface. Measurements of parallel ion flux were used to estimate the radial dimension of the cylindrical shell interface. Energy arguments were used to estimate the parallel ion drift speed in the inner and outer plasmas. The D'Angelo instability [D'Angelo, 1965], which is destabilized by shear in the parallel ion velocity, was studied using this configuration [D'Angelo and von Goeler, 1966; Willig et al., 1997; Merlino et al, 1997]. Willig et al. [1997] and Merlino et al. [1997] used a pair of single-sided Langmuir probes joined back to back to obtain separate but simultaneous values of the upstream and the downstream flux at specific points in the plasma column. Their flux measurements were interpreted as resulting from two counter-streaming ion beams, both of which are detected at every point across the plasma. Their velocities are not referenced to the direction of the magnetic field, preventing a determination of the sign of the fluid parallel-velocity shear from their report. 
Agrimson et al. [2001] proposed another way of producing sheared parallel ion flow in a double-ended Q machine. Using a Q machine in which the "magnetic-field lines diverged from the west hot plate to the east hot plate" [Agrimson et al., 2001], they mapped the plasma produced at the larger-magnetic-field end to a ring electrode surrounding the ionizer at the low-magnetic-field end. The plasma produced at the smaller-magnetic-field end mapped to the inner position of the hot plate at the larger-magnetic-field end. By adjusting the ionizers' heating power and by applying positive voltage to the electrode, they established zero-ionflow conditions in the outer plasma, as determined with the aid of two single-sided Langmuir probes. At smaller radii the net parallel ion flux was directed away from the hot plate with the larger heating power.

By segmenting concentrically the hot plate, using it exclusively for producing ions and accelerating the ions in an electron-free region between the hot plate and a negatively biased mesh a centimeter or so away, Odaka et al. [2000] demonstrated experimental control of the radial profile of the ion's parallel energy distribution function in a double-ended Q-machine. Using a different bias voltage on inner and outer segments of one ionizer, they produced concentric ion beams, as measured with a directional electrostatic energy analyzer probe. Neither ion beam showed variation in the beam energy as a function of radius, but for both beams, the beam density dropped to a negligible value within an ion gyroradius of the edge of the producing segment. For beam velocities exhibiting no shear, radial profiles of the beam density could be generated by fixing the energy of analyzer's repelling or retarding voltage and plotting the collector current as a function of radius.

Laser-induced fluorescence was first used by Skiff and Fasoli [1993] to document parallel-ion-velocity shear in a barium plasma. Shear was induced by introducing a massive inert gas (xenon) into the vacuum chamber. When the neutral pressure exceeded $10^{-4}$ Torr, the parallel flow became sheared. Variations in the parallel-ion-velocity of the order of the sound speed were reported, with $d v_{d i} / d x \sim 1(\mathrm{~ms})^{-1}$. When using low-atomic-mass gases, the parallel flow remained spatially uniform irrespective of the neutrals pressure used.

Koepke et al. [2000] used laser-induced fluorescence to measure the parallel ion velocity in the "ring and disk" configuration of a double-ended Q-machine. The parallel ion velocity distribution revealed two cold, counter-streaming ion populations, neither of which exhibited shear in the parallel drift velocity. Analyzing the two populations as a single drifting-Maxwellian distribution in flux-averaged fashion yielded flux-averaged drift velocity 
and parallel-velocity shear that were drastically different from those values for either of the two populations themselves [Koepke et al., 2002]. The flux-averaged velocity profile underestimated the actual drift speeds and overestimated the actual shear.

\section{I.A.2 Inhomogeneous ion flow in space}

Evidence for shear in parallel ion flow in space was reported decades ago based on OGO satellite observations [D'Angelo, 1973], HEOS satellite observations [D'Angelo et al.,1974], AE-C satellite observations [Potemra et al. 1978] and DE-2 satellite observations [Heelis et al., 1984, Loranc et al.,1991]. More recent evidence of sheared parallel ion flow comes from the FAST satellite [McFadden et al, 1998]. When crossing an auroral arc, the FAST satellite observed a single drifting-ion population with a drift speed and beam density that varied by a large fraction over length scales as short as $5 \mathrm{~km}$ (10-100 ion gyroradii, depending on the species, $\mathrm{H}^{+}, \mathrm{He}^{+}$or $\mathrm{O}^{+}$) perpendicular to the magnetic field [McFadden et $a l ., 1998]$. In dimensionless terms, the parallel-velocity shear $\left(\omega_{\mathrm{ci}}{ }^{-1} d v_{d i} / d x\right)$ for the typical keV ion beam could be as large as $\omega_{\mathrm{ii}}^{-1} \Delta v / \Delta x=\omega_{\mathrm{i}}^{-1}\left(60 v_{\mathrm{ti}}\right) /\left(10-100 \rho_{i}\right)=(0.6-6)$, with the highest values of the normalized shear corresponding to the $\mathrm{O}^{+}$normalization [Amatucci, 1999], lending substantial merit to the idea that shear-modified ion acoustic or shear-modified ion cyclotron waves can exist in the Earth's auroral zone.

\section{I.B Basic plasma physics of electrostatic ion waves}

Modern plasma physics began in the late 1920's with the work of Langmuir and coworkers [Tonks and Langmuir, 1929] on plasma sheaths and instabilities. Innumerable breakthrough discoveries have led to increasingly sophisticated experiments and theoretical models. Plasma physics is important in understanding planetary magnetosphere, stellar worlds and outer space, and has been the cornerstone of the effort toward harnessing fusion energy in reactors. In all cases, instabilities can arise spontaneously, sometimes having a significant effect on the plasma. 
Oscillations that grow (decay) exponentially in space or time are considered unstable (damped). Various mechanisms leading to plasma instability have been identified over the decades, each one being associated with a particular source or combination of sources of free energy that overcomes any inherent dissipation in the plasma. Damping is associated with dissipation, such as collisions, or with collisionless wave-phase particle resonance, such as phenomena involving primarily particles moving at the phase velocity of the oscillations.

Coherent, periodic oscillations of the plasma parameters are referred to as plasma waves. The Fourier representation of a plasma wave identifies with it a specific value of $\omega$ and $\boldsymbol{k}$ and assumes the wave amplitude extends over all space and time. The concept of a wave packet is applied to the case in which the wave-amplitude profile has a finite extent in space or time. An example of a plasma wave is the traveling acoustic oscillations known as the ion acoustic wave, when ions and electrons oscillate along the magnetic field lines.

At low frequency are ion-related instabilities in which the ions participate dynamically. At high frequency are the electron instabilities in which large ion inertia prevents the positive background charge distribution from being perturbed.

\section{I.C Electrostatic ion cyclotron and ion acoustic waves in the presence of parallel ion velocity shear}

D'Angelo [1965] showed that a low frequency, non-resonant instability arises when the parallel ion drift velocity varies in the perpendicular (to the magnetic field) direction. This instability was initially named by D'Angelo “Kelvin-Helmholtz instability". Later, Ganguli et al. [1996] showed that the instability has a different nature than the Kelvin-Helmholtz instability, and proposed the name the "D'Angelo instability". Petviashvili [1967] and Mikhailovskii [1974] found results on the ion-acoustic branch of the instability similar to D'Angelo's. Mikhailovskii [1992] also showed that depending on the sign of ratio between the perpendicular and the parallel wavevectors, two types of "slow magnetoacoustic KelvinHelmholtz instabilities" [Mikhailovskii, 1992] can be excited. One is referred to as the "ionacoustic Kelvin-Helmholtz instability" and the other one is its electromagnetic branch, differing by the magnitude of the growth rates. The existence of very-low-frequency 
instabilities $\left(\omega<<k_{z} v_{t i}\right)$ corresponding to the (purely growing) D'Angelo instability was also predicted. The effects of density and temperature gradients are also analyzed.

A general kinetic dispersion relation including the effects of velocity inhomogeneity both in the parallel and perpendicular directions was derived by Ganguli et al. [1994]. This dispersion was applied by Gavrishchaka, et al. [1998, 2000] to show that ion cyclotron or ion acoustic waves could be destabilized in the presence of inhomogeneous ion flow. They showed that the presence of sheared parallel ion flow could be a source of free energy for these waves, so that the waves could grow spontaneously in inhomogeneous-parallel-ion-flow plasmas with or without relative parallel electron drift. Moreover, in the case of shearmodified ion cyclotron (SMIC) waves, the ubiquitous ion cyclotron damping is theoretically shown to be inverted, i.e., wave destabilizing instead of wave stabilizing [Ganguli et al., 2002]. Shear-modified ion acoustic (SMIA) waves are the low-frequency $(n=0)$ manifestation of the shear-modified ion cyclotron waves. The model predicts a much smaller electron drift necessary for SMIA waves destabilization, compared to the electron drift that destabilizes ionacoustic waves in homogeneous-flow plasmas. For both type of waves, the model does not require electron drift for wave excitation in the presence of large shear.

Experimental confirmation of the role of parallel-velocity shear in destabilizing ion acoustic waves and experimental investigations of the role of parallel-velocity shear in destabilizing multi-harmonic ion cyclotron waves, respectively, were initially reported by Agrimson et al. [2001, 2002]. More detailed experimental confirmation of SMIA waves growing spontaneously in inhomogeneous parallel ion flow was presented by Teodorescu et al. [2002a]. The effect of ion temperature anisotropy in the growth and propagation of SMIA waves was experimentally studied by Teodorescu et al. [2003]. In addition, inverse ion cyclotron damping and multi-harmonic ion cyclotron waves destabilization in sheared parallel ion flow plasmas were experimentally documented by Teodorescu et al. [2002b].

The effect of ion and electron temperature anisotropy in the SMIA wave growth and propagation was first addressed theoretically [Spangler, 2001; Spangler et al., 2002]. The trend of increasing growth rate, decreasing propagation angle, and increasing wave frequency in the case of increasing ion temperature anisotropy $T_{i y} / T_{i z}>1$ was found. The model showed that in the case that larger anisotropies are achieved by decreasing ion parallel temperature (while keeping $T_{i y}$ and electron parallel temperature $T_{e z}$ fixed) the increase in wave growth rate and wave frequency, along with the decrease in the propagation angle, are rather small (of 
the order of 50-70\%). On the other hand, the model showed that if larger anisotropies are achieved by increasing both $T_{i y}$ and $T_{e z}$ (while keeping the ion parallel temperature fixed), the increase in the wave growth rate and frequency, along with the decrease in the wave propagation angle, are dramatic (around 500\% for the same increase in ion temperature anisotropy).

\section{I.D Scope of the dissertation}

The work presented in this dissertation represents an experimental study of parallel and perpendicular ion and electron flow in a $\mathrm{Q}$ machine. The initial effort was directed towards creating shear in the parallel velocity of a single, drifting-Maxwellian ion population produced in a $\mathrm{Q}$ machine. The data document two ways shear in the parallel ion flow can be produced, controlled and non-invasively measured. The $\mathrm{Q}$ machine had been already configured to be used either in the double-ended or in the single-ended configuration. Several technological improvements were made to the plasma-sources. A complex laser-induced fluorescence apparatus had also been previously set up to allow for measurements of both parallel and perpendicular ion velocity distributions [Koepke et al., 1998; Koepke et al., 2000]. Computer programs were created in order to analyze the laser-induced fluorescence measurements data. New probes and data-analyzing programs were developed and used in studying the plasma and wave properties.

Electrostatic ion cyclotron waves, which spontaneously arose when parallel electron drift and parallel-ion-velocity shear were combined, are characterized. Parallel-shear-modified ion cyclotron waves are identified based on agreement with the model. Evidence for reducing and inverting ion cyclotron damping by applying parallel-ion-velocity shear, observing the spontaneously excited waves and comparing the results to a model [Gavrishchaka et al, 2000; Ganguli et al., 2002] is presented. Multi-harmonic ion cyclotron waves with frequencies as large as the lower-hybrid frequency are measured.

Ion acoustic waves that spontaneously arose when parallel electron drift and parallelion-velocity shear were combined are characterized. Parallel-shear-modified ion acoustic waves are identified and the model [Gavrishchaka et al, 1998, 1999] is benchmarked. Wave propagation characteristics are fully determined. The effect of ion-temperature anisotropy on 
the growth and propagation of shear-modified ion-acoustic waves is experimentally described and shown to be consistent with the generalized model that includes anisotropy [Spangler et al., 2002].

The results presented in this work improve the interpretation of phenomena observed in the ionosphere, where broadband, low frequency waves are known to exist. But the results also broaden fundamental plasma physics concepts such as ion-cyclotron damping to include the possibility of inverse ion-cyclotron damping. 


\section{Chapter II. Theory}

This chapter presents the fundamental concepts and the experimentally-relevant properties of shear-modified ion cyclotron (SMIC) waves and shear-modified ion acoustic (SMIA) instability waves described by Ganguli et al. [1994], Gavrishchaka et al. [1998, 1999, 2000] and Ganguli et al. [2002]. The derivation of the dispersion relation is presented using isotropic, magnetized-plasma, kinetic theory. This treatment follows Spangler's [2001] derivation for anisotropic plasma, but in the isotropic limit. The effect of ion temperature anisotropy on the propagation and growth of SMIA waves is discussed.

\section{II.A Electrostatic ion cyclotron waves modified by shear in the parallel-ion-velocity}

Destabilization of ion cyclotron waves in plasma containing inhomogeneous parallel ion flow has been studied theoretically for the isotropic [Gavrishchaka et al., 2000; Ganguli et al., 2002] and anisotropic [Spangler, 2001; Spangler et al., 2002] cases. The dispersion relation was found using the linearized Vlasov equation and Poisson's equation.

The ion velocity distribution is constructed of the constants of motion in the isotropic plasma limit $\left(T_{s||}=T_{s \perp}, s=\right.$ species). The basic kinetic theory equations relevant to the SMIC waves are the linearized Vlasov equation

$$
\frac{\partial f}{\partial t}+\mathbf{v} \cdot \nabla f+\frac{q}{m}(\mathbf{E}+\mathbf{v} \times \mathbf{B}) \cdot \nabla_{\mathbf{v}} f=0
$$

and the Poisson equation

$$
\nabla \cdot \mathbf{E}_{1}=4 \pi \sum_{s} \rho_{1 s}
$$

Linearization allows separating the terms in time-independent and time-dependent parts, respectively. Any quantity $A(\mathbf{x}, t)$ is thus written

$$
A(\mathbf{x}, t)=A_{0}(\mathbf{x})+A_{1}(\mathbf{x}, t)
$$


where all perturbation quantities $A_{1}(\boldsymbol{x}, t)$ are Fourier decomposed as

$$
A_{1}(\mathbf{x}, t)=\sum_{k} A_{k} \exp (i \mathbf{k} \cdot \mathbf{x}-i \omega t)
$$

where $A_{k}$ is the amplitude corresponding to the wavevector $\boldsymbol{k}$ and $\boldsymbol{\omega}(\boldsymbol{k})$ is the frequency of the plane wave.

The zeroth-order Vlasov equation is

$$
\frac{\partial f_{0}}{\partial t}+\mathbf{v} \cdot \nabla f_{0}+\frac{q}{m}\left(\mathbf{E}_{0}+\mathbf{v} \times \mathbf{B}_{0}\right) \cdot \nabla_{\mathbf{v}} f_{0}=0
$$

and the first-order Vlasov equation is given by

$$
\frac{\partial f_{1}}{\partial t}+\mathbf{v} \cdot \nabla f_{1}+\frac{q}{m}\left(\mathbf{E}_{0}+\mathbf{v} \times \mathbf{B}_{0}\right) \cdot \nabla_{\mathbf{v}} f_{1}+\frac{q}{m}\left(\mathbf{E}_{1}+\mathbf{v} \times \mathbf{B}_{1}\right) \cdot \nabla_{\mathbf{v}} f_{0}=0 .
$$

The instabilities discussed here are electrostatic, $\mathbf{B}_{1}=0$, and there is no external electric field applied, $\boldsymbol{E}_{\boldsymbol{0}}=0$, thus all first order interactions are due to the interaction between the charge $q$ and the first order perturbed electric field $\boldsymbol{E}_{\boldsymbol{l}}$.

From (15) it follows that

$$
f_{1}(\mathbf{v}, t)=-\frac{q}{m} \int_{-\infty}^{t} \mathbf{E}_{1} \cdot \nabla_{\mathbf{v}} f_{0} d t^{\prime}
$$

Equation (16) is always satisfied if the zeroth order distribution function $f_{0}(v)$ is a function of the constants of motion. The constants of motion are

$$
\begin{aligned}
& m=\text { mass of particle; } \omega_{c s}=\frac{q_{s} B}{m_{s}} \text { species angular gyrofrequency } \\
& x_{g}=\frac{v_{y}}{\omega_{c s}}+x \quad(x \text { is the guiding center position })
\end{aligned}
$$


$y_{g}=\frac{v_{x}}{\omega_{c s}}-y \quad(y$ is the guiding center position $)$

$v_{z}$ (species parallel velocity)

$\frac{1}{2} k_{B} T_{z}=\frac{1}{2} m v_{z}^{2} \quad$ (species parallel kinetic energy)

$\sqrt{v_{x}^{2}+v_{y}^{2}}=v_{\perp}$ (species perpendicular velocity)

$k_{B} T_{\perp}=\frac{1}{2} m\left(v_{x}^{2}+v_{y}^{2}\right)$ (species perpendicular kinetic energy).

Here, $s=i$,e denotes the species (ion or electron, respectively). Using these constants of motion, the unperturbed velocity-distribution function $f_{0}$ is

$$
f_{0}\left(h_{\|}, h_{\perp}, x_{g}\right)=C \exp \left[-2 \alpha\left(h_{\|}\left(x_{g}\right)+h_{\perp}\right)\right]
$$

where

$$
\begin{aligned}
& h_{\|}\left(x_{g}\right)=\frac{\left(v_{d}-v_{d 0}\left(x_{g}\right)\right)^{2}}{2}, \\
& h_{\perp}=\frac{v_{x}^{2}+v_{y}^{2}}{2} \equiv \frac{v_{\perp}^{2}}{2}, \\
& \alpha=\frac{1}{2 v_{t}^{2}}, v_{t}^{2}=\frac{k_{B} T}{m},\left.v_{\mathrm{d} 0}\left(x_{\mathrm{g}}\right) \equiv v_{\mathrm{d}}\left(x_{\mathrm{g}}\right)\right|_{x=0} \text { and } \\
& C=\frac{n_{0}}{\pi^{3 / 2}} \alpha^{3 / 2} .
\end{aligned}
$$

Here, $n_{0}$ is the unperturbed part of the radially-uniform plasma density. The parallel flow is inhomogeneous in the perpendicular $x$ direction, i.e.,

$$
v_{d}\left(x_{g}\right)=v_{d 0}\left(x_{g}\right)+\frac{d v_{d}\left(x_{g}\right)}{d x} \Delta x,
$$

where $v_{d}$ is the parallel drift velocity of the species. Thus, 


$$
\begin{aligned}
\nabla_{\mathbf{v}} f_{0} & =\frac{\partial f_{0}}{\partial h_{\|}} \frac{\partial h_{\|}}{\partial \mathbf{v}}+\frac{\partial f_{0}}{\partial h_{\perp}} \frac{\partial h_{\perp}}{\partial \mathbf{v}}+\frac{\partial f_{0}}{\partial x_{g}} \frac{\partial x_{g}}{\partial \mathbf{v}}= \\
& =-2 f_{0}\left[\alpha \mathbf{v}-\alpha \mathbf{v}_{d}+\alpha\left(v_{d}-v_{d 0}\left(x_{g}\right)\right)\left(\hat{z}-\frac{d v_{d}\left(x_{g}\right)}{\omega_{c s} d x} \hat{y}\right)\right]
\end{aligned}
$$

The electrostatic limit of the SMIC instability is considered, $\boldsymbol{B}_{1}=0$, and so $\mathbf{E}_{1}=-\nabla \phi_{1}$ where $\phi_{1}=\sum_{k} \phi_{1 k} \exp (i \mathbf{k} \cdot \mathbf{x}-i \omega t)$.

Expressing $f_{1}(\mathbf{v}, t)=\sum_{k} f_{1 k} \exp (i \mathbf{k} \cdot \mathbf{x}-i \omega t)$ from (16) it follows that

$$
\begin{aligned}
f_{1 k}(\mathbf{v})=- & 2 f_{0} \frac{q}{m}\left\{\left.\alpha \phi_{1 k} \exp \left(\mathbf{k} \cdot\left(\mathbf{r}^{\prime}-\mathbf{r}\right)-\omega\left(t^{\prime}-t\right)\right) i\right|_{-\infty} ^{t}+\right. \\
& +\int_{-\infty}^{t}\left[i\left(\alpha\left(\omega-k_{z} v_{d}\right)+\alpha\left(v_{d}-v_{d}\left(x_{g}\right)\right)\left(k_{z}-k_{y} \frac{d v_{d}}{\omega_{c s} d x}\right)\right) \times\right. \\
& \left.\times \phi_{1 k} \exp \left(\left(\mathbf{k} \cdot\left(\mathbf{r}^{\prime}-\mathbf{r}\right)-\omega\left(t^{\prime}-t\right)\right) i\right] d t^{\prime}\right\} .
\end{aligned}
$$

Denoting the particle's angular position at time $t$ with $\varphi=\omega_{c s} t+\varphi_{0}$ and using $\tau=t-t^{\prime}$, (22) can be rewritten using the solution of the unperturbed orbits.

Knowing that the uniform magnetic field is $\boldsymbol{B}=B_{0} \boldsymbol{z}$, the unperturbed orbit for a charged particle is

$$
\begin{aligned}
& x(t)=x\left(t^{\prime}\right)+\frac{v_{\perp}}{\omega_{c s}}\left[\sin \left(\omega_{c s} \tau-\varphi\right)+\sin \varphi\right], \\
& y(t)=y\left(t^{\prime}\right)+\frac{v_{\perp}}{\omega_{c s}}\left[\cos \left(\omega_{c s} \tau-\varphi\right)-\cos \varphi\right], \text { and } \\
& z(t)=z\left(t^{\prime}\right)+v_{d} \tau
\end{aligned}
$$

and

$$
\begin{aligned}
& v_{x}(t)=v_{\perp} \cos \left(\omega_{c s} \tau-\varphi\right), \\
& v_{y}(t)=-v_{\perp} \sin \left(\omega_{c s} \tau-\varphi\right) \quad, \text { and } \\
& v_{z}(t) \equiv v_{d}(t)=v_{d 0}+\frac{d v_{d}}{d x} \Delta x .
\end{aligned}
$$

Now, it follows that 


$$
\begin{aligned}
i \mathbf{k} \cdot\left(\mathbf{r}^{\prime}-\mathbf{r}\right) & =i \frac{k_{\perp} v_{\perp}}{\omega_{c s}}\left[\sin \beta\left(\sin \left(\omega_{c s} \tau-\varphi\right)-\sin \varphi\right)+\cos \beta\left(\cos \left(\omega_{c s} \tau-\varphi\right)-\cos (\varphi)\right)\right]- \\
& -i k_{z} v_{d}=i \frac{k_{\perp} v_{\perp}}{\omega_{c s}}\left[\sin \left(\omega_{c s} \tau-\varphi+\beta\right)-\sin (\varphi+\beta)\right]-i k_{z} v_{d} \tau
\end{aligned}
$$

where $k_{x}=k_{\perp} \cos \beta$ and $k_{y}=k_{\perp} \sin \beta$ is used. Inserting into (22), the expression for the perturbed distribution becomes

$$
\begin{aligned}
f_{1 k}\left(v_{d}, v_{\perp}\right)= & -2 f_{0} \frac{q}{m}\left[\alpha \phi_{1 k}-i\left(\alpha\left(\omega-k_{z} v_{d}\right)+\alpha\left(v_{d}-v_{d 0}\left(x_{g}\right)\right)\left(k_{z}-k_{y} \frac{d v_{d}}{\omega_{c s} d x}\right)\right) \times\right. \\
& \left.\times \int_{0}^{2 \pi} d \varphi \int_{-\infty}^{0} \exp \left[\frac{k_{\perp} v_{\perp}}{\omega_{c s}}\left(\sin \left(\omega_{c s} \tau-\varphi+\beta\right)-\sin (\varphi+\beta)\right)+\left(\omega-k_{z} v_{d}\right) \tau\right] i d \tau\right]
\end{aligned}
$$

where $v_{\perp}=\sqrt{v_{x}^{2}+v_{y}^{2}}$ and $k_{\perp}=\sqrt{k_{x}^{2}+k_{y}^{2}}$.

Using the generating function for the Bessel functions $J_{n}(b)$

$$
e^{i b \sin \theta}=\sum_{n=-\infty}^{\infty} e^{i n \theta} J_{n}(b)
$$

the integration over $\varphi$ and $\tau$ in (26) can be done by first integrating over $\tau$ and then over $\varphi$.

$$
\begin{aligned}
& \int_{-\infty}^{0} e^{i \frac{k_{\perp} v_{\perp}}{\omega_{c s}}\left(\sin \left(\omega_{c i} \tau-\varphi-\beta\right)-\sin (\varphi+\beta)\right)+\left(\omega-k_{z} v_{d}\right) \tau} d \tau=\int_{-\infty}^{0} e^{i \frac{k_{\perp} v_{\perp}}{\omega_{c s}} \sin \left(\varphi+\beta+\frac{\pi}{2}\right) \sin \omega_{c s} \tau} \times \\
& \times e^{-i \frac{k_{\perp} v_{\perp}}{\omega_{c s}} \sin (\varphi+\beta) \sin \left(\omega_{c i} \tau+\frac{\pi}{2}\right)} e^{i \frac{k_{\perp} v_{\perp}}{\omega_{c s}}\left(\omega-k_{z} v_{d}\right) \tau} d \tau= \\
& =\sum_{n=-\infty}^{\infty} e^{i n\left(\varphi+\beta+\frac{\pi}{2}\right)} e^{-i n(\varphi+\beta)} J_{n}^{2}\left(\frac{k_{\perp} v_{\perp}}{\omega_{c s}}\right) \int_{-\infty}^{0} e^{i n \omega_{c s} \tau} e^{-i n\left(\omega_{c s} \tau+\frac{\pi}{2}\right)} e^{i\left(\omega-k_{z} v_{d}\right) \tau} d \tau= \\
& =\sum_{n} J_{n}^{2}\left(\frac{k_{\perp} v_{\perp}}{\omega_{c s}}\right) \frac{1}{\omega-n \omega_{c s}-k_{z} v_{d}}
\end{aligned}
$$

and so,

$$
\int_{0}^{2 \pi} d \varphi \int_{-\infty}^{0} \exp \left[\frac{k_{\perp} v_{\perp}}{\omega_{c s}}\left(\sin \left(\omega_{c s} \tau-\varphi+\beta\right)-\sin (\varphi+\beta)\right)+\left(\omega-k_{z} v_{d}\right) \tau\right] i d \tau=
$$


$=\sum_{n} J_{n}^{2}\left(\frac{k_{\perp} v_{\perp}}{\omega_{c s}}\right) \frac{2 \pi}{\omega-n \omega_{c s}-k_{z} v_{d}}$.

Thus, the perturbed distribution becomes:

$$
\begin{aligned}
f_{1 k}\left(v_{d}, v_{\perp}\right) & =-\frac{4 \pi q f_{0}}{m} \alpha \phi_{1 k}\left[1-\sum_{n=-\infty}^{\infty}\left(J_{n}\left(\frac{k_{\perp} v_{\perp}}{\omega_{c s}}\right)\right)^{2} \times\right. \\
& \left.\times\left(\frac{\left(\omega-k_{z} v_{d}+\left(v_{d}-v_{d o}\left(x_{g}\right)\right)\left(k_{z}-k_{y} \frac{d v_{d}}{\omega_{c s} d x}\right)\right.}{\omega-n \omega_{c s}-k_{z} v_{d}}\right)\right] .
\end{aligned}
$$

This expression of the perturbed part of the velocity distribution, in which the unperturbed distribution $f_{0}$ is given by (14), is used to determine the perturbed density $n_{l}$ where

$$
n_{1 k}=\int_{-\infty}^{\infty} \int_{0}^{\infty} f_{1 k}\left(v_{d}, v_{\perp}\right) d v_{d} d v_{\perp} .
$$

The integration over the perpendicular velocity $v_{\perp}$ is done in the "local approximation" limit: the shear layer $L$ is much larger than the ion gyroradius $\rho_{i} \equiv v_{t i} / \omega_{c i}$. It is necessary to use this method since there are no analytic methods to integrate expressions like

$$
\int_{0}^{\infty} e^{-\infty v_{\perp}^{2}} v_{\perp} d v_{\perp}
$$

if the dependence on $v_{y}$ is kept in the expression of the guinding center $x_{g}$, which in turn is a parameter of the parallel drift velocity $v_{\mathrm{d}}$. Therefore, the drift velocity is expressed as a Taylor series

$$
v_{d}\left(x_{g}\right)=v_{d}\left(x+x_{g}-x\right)=v_{d}\left(x+\rho_{i}\right)=v_{d}(x)+\mathrm{O}\left(\rho_{i} / L\right)+\ldots
$$

and all terms of order $\left(\rho_{i} / L\right)$ are neglected so that $v_{\mathrm{d}}\left(x_{\mathrm{g}}\right) \approx v_{\mathrm{d}}(x)$. In the experiments described in this work, the shear layer was measured and compared with the ion gyroradius yielding in most of the cases $0.2<\rho_{i} / L<0.6$; thus this approximation is considered valid.

Then

$$
\int_{0}^{\infty} e^{-\alpha \nu_{\perp}^{2}} v_{\perp} d v_{\perp}=\frac{1}{2 \alpha}
$$


and

$$
\int_{0}^{\infty}\left(J_{n}\left(\frac{k_{\perp} v_{\perp}}{\omega_{c s}}\right)\right)^{2} e^{-\alpha v_{\perp}^{2}} v_{\perp} d v_{\perp}=\frac{1}{2 \alpha} \Gamma_{n}(b),
$$

where $b=k_{\perp}^{2}\left(2 \alpha \omega_{c s}^{2}\right)^{-1} \equiv\left(k_{\perp} \rho_{s}\right)^{2}, \Gamma_{n}(b)=I_{n}(b) e^{-b}$, and $I_{n}(b)$ is the modified Bessel function [Wu and Zmuidzinas, 1969].

The integration over the parallel velocity $v_{d}$ of the parallel-velocity-dependent perturbed distribution $f_{1 k}$,

$$
\begin{aligned}
f_{1 k}\left(v_{d}\right) & =-\frac{2 q n_{0} \alpha^{3 / 2}}{\sqrt{\pi} m} \varphi_{1 k} e^{-2 \alpha\left(v_{d}-v_{d 0}\right)^{2}}\left[1-\sum_{n=-\infty}^{\infty} \Gamma_{n}(b) \times\right. \\
& \left.\times\left(\frac{\left(\omega-k_{z} v_{d}\right)+\left(v_{d}-v_{d 0}\right)\left(k_{z}-k_{y} \frac{d v_{d}}{\omega_{c s} d x}\right)}{\omega-n \omega_{c s}-k_{z} v_{d}}\right)\right],
\end{aligned}
$$

is done in two parts and the following results are obtained.

$$
\int_{-\infty}^{\infty} e^{-\alpha\left(v_{d}-v_{d 0}\right)^{2}} d v_{d}=\sqrt{\frac{\pi}{\alpha}}
$$

and

$$
\begin{gathered}
\int_{-\infty}^{\infty}\left(\frac{\left(\omega-k_{z} v_{d}\right)+\left(v_{d}-v_{d 0}\right)\left(k_{z}-k_{y} \frac{d v_{d}}{\omega_{c s} d x}\right)}{\omega-n \omega_{c s}-k_{z} v_{d}}\right) e^{-\alpha\left(v_{d}-v_{d 0}\right)^{2}} d v_{d}= \\
=-\frac{1}{\sqrt{\alpha}} \int_{-\infty}^{\infty} \frac{\frac{\sqrt{\alpha}}{k_{z}}\left(\omega-k_{z} v_{d}\right)-a \frac{k_{y}}{k_{z}} \frac{d v_{d}}{\omega_{c s} d x}}{a-\frac{\sqrt{\alpha}}{k_{z}}\left(\omega-n \omega_{c s}-k_{z} v_{d}\right)} e^{-a^{2}} d a
\end{gathered}
$$

where the substitution $a=\alpha^{1 / 2}\left(v_{d}-v_{d 0}\right)$ is used. The latter form of the integral is expressed using the plasma dispersion function

$$
Z\left(\xi_{n}\right)=\frac{1}{\pi^{1 / 2}} \int_{-\infty}^{\infty} \frac{e^{-a^{2}}}{a-\xi_{n}} d a
$$

with

$$
\xi_{n}=\frac{\omega-k_{z} v_{d}(x)-n \omega_{c s}}{\sqrt{2} k_{z} v_{t s}}
$$


Thus,

$$
-\frac{1}{\sqrt{\alpha}} \int_{-\infty}^{\infty} \frac{\frac{\sqrt{\alpha}}{k_{z}}\left(\omega-k_{z} v_{d}\right)-a \frac{k_{y}}{k_{z}} \frac{d v_{d}}{\omega_{c s} d x}}{a-\frac{\sqrt{\alpha}}{k_{z}}\left(\omega-n \omega_{c s}-k_{z} v_{d}\right)} e^{-a^{2}} d a \equiv-\sqrt{\frac{\pi}{\alpha}}\left\{\xi_{0} Z\left(\xi_{n}\right)-\frac{k_{y}}{k_{z}} \frac{d v_{d}}{\omega_{c s} d x}\left(1+\xi_{n} Z\left(\xi_{n}\right)\right)\right\} .
$$

After integrating over the parallel velocity $v_{d}$, the perturbed density $n_{l k}$ is found from

$$
\begin{aligned}
n_{1 k} & =\int_{-\infty 0}^{\infty} \int_{1 k}^{\infty} f_{1 k}\left(v_{d}, v_{\perp}\right) d v_{d} d v_{\perp}= \\
& =-\frac{2 n_{0} \alpha}{m} \varphi_{1 k}\left\{1+\sum_{n=-\infty}^{\infty} \Gamma_{n}(b)\left[\xi_{0} Z\left(\xi_{n}\right)-\frac{k_{y}}{k_{z} \omega_{c s}} \frac{d v_{d}}{d x}\left(1+\xi_{n} Z\left(\xi_{n}\right)\right]\right\} .\right.
\end{aligned}
$$

Both the ion and the electron density perturbation terms, $n_{1 \mathrm{i}}$ and $n_{1 \mathrm{e}}$, respectively, are found using this treatment. They are then used in the Poisson's equation

$$
\begin{gathered}
\nabla \cdot \mathbf{E}_{1}=4 \pi e\left(n_{1 i}-n_{1 e}\right), \\
\frac{k^{2} \varphi_{1 k}}{4 \pi e}=-\frac{2 e n_{0}}{\varepsilon_{0}} \varphi_{1 k}\left\{\frac{1}{2 v_{t i}^{2}}+\frac{1}{2 v_{t i}^{2}} \sum_{n=-\infty}^{\infty} \Gamma_{n}\left(b_{i}\right)\left[\xi_{0 i} Z\left(\xi_{n i}\right)-\frac{k_{y}}{\omega_{c i} k_{z}} \frac{d v_{d i}}{d x}\left(1+\xi_{n i} Z\left(\xi_{n i}\right)\right)\right]-\right. \\
\left.-\frac{1}{2 v_{t e}^{2}}-\frac{1}{2 v_{t e}^{2}} \sum_{n=-\infty}^{\infty} \Gamma_{n}\left(b_{e}\right)\left[\xi_{0 e} Z\left(\xi_{n e}\right)-\frac{k_{y}}{\omega_{c e} k_{z}} \frac{d v_{d e}}{d x}\left(1+\xi_{n e} Z\left(\xi_{n e}\right)\right)\right]\right\} .
\end{gathered}
$$

Here, $v_{\mathrm{di}}$ and $v_{\mathrm{de}}$ are the ion and electron drift velocities, respectively, in the laboratory frame.

In the limit $\omega<<\omega_{c e}$, where $\omega$ is the wave angular frequency in the laboratory frame, $n=0$ for electrons and, transforming to the ion frame, the dispersion relation in an isotropic plasma is found to be

$$
\sum_{n=-\infty}^{\infty} \Gamma_{n}(b) F_{n i}+\frac{T_{i}}{T_{e}}\left(1+F_{0 e}\right)+k^{2} \lambda_{d i}^{2}+1=0
$$

where

$$
\begin{aligned}
& \omega_{\mathrm{1}}=\omega-k_{\mathrm{z}} v_{\mathrm{di}} \\
& F_{n, i}=\frac{\omega_{1}}{\sqrt{2}\left|k_{z}\right| v_{t z, i}} Z\left(\frac{\omega_{1}-n \omega_{c i}}{\sqrt{2}\left|k_{z}\right| v_{t z, i}}\right)-\frac{d v_{d i}}{u \omega_{c i} d x}\left(1+\frac{\omega_{1}-n \omega_{c i}}{\sqrt{2}\left|k_{z}\right| v_{t z, i}} Z\left(\frac{\omega_{1}-n \omega_{c i}}{\sqrt{2}\left|k_{z}\right| v_{t z, i}}\right)\right) \\
& \lambda_{d i}^{2}=\frac{m_{i} v_{t i}^{2}}{4 \pi n_{0} e^{2}} \text { is the ion Debye length, } v_{\mathrm{d}}=v_{\mathrm{de}}-v_{\mathrm{di}}, u=k_{\mathrm{z}} / k_{\mathrm{y}} \text { and }
\end{aligned}
$$




$$
\begin{aligned}
& F_{0, e}=\frac{1}{\sqrt{2}\left|k_{z}\right| v_{t z, i}}\left(\omega_{1}-k_{z} v_{d}(x)\right) Z\left(\frac{\omega_{1}-k_{z} v_{d}(x)}{\sqrt{2}\left|k_{z}\right| v_{t z, e}}\right)+\frac{d v_{d e}}{d x} \frac{1}{u \omega_{c e}} \times \\
& \times\left(1+\frac{\omega_{1}-k_{z} v_{d}(x)}{\sqrt{2}\left|k_{z}\right| v_{t z, e}} Z\left(\frac{\omega_{1}-k_{z} v_{d}(x)}{\sqrt{2}\left|k_{z}\right| v_{t z, e}}\right)\right) .
\end{aligned}
$$

This is the multi-harmonic electrostatic ion cyclotron waves (in the presence of parallelvelocity shear) dispersion relation of Gavrishchaka et al. [2000].

Of particular importance here are the wave growth conditions. The analysis of the imaginary part of the dispersion relation (44) shows that the presence of the shear term influences the wave growth. Setting the imaginary part of (44) equal to zero and using the marginal stability condition $(\gamma=0)$, it follows that

$D_{i} \equiv \sum_{n} \Gamma_{n}(b)\left[\xi_{0 i}-\xi_{n i} \frac{d v_{d i} / d x}{u \omega_{c i}}\right] \operatorname{Im}\left(Z\left(\xi_{n i}\right)\right)+\frac{T_{i}}{T_{e}}\left(1+\frac{d v_{d e} / d x}{u \omega_{c e}}\right) \xi_{e} \operatorname{Im}\left(Z\left(\xi_{e}\right)\right)=0$.

In the $\xi_{n i}>1$ and $\xi_{e}<<1$ limits (appropriate if $v_{d}<<v_{t e}$ ), (46) becomes

$$
\sum_{n} \Gamma_{n}(b)\left[1-\left(1-\frac{n \omega_{c i}}{\omega_{1 r}}\right) \frac{d v_{d i} / d x}{u \omega_{c i}}\right] e^{-\frac{\left(\omega_{1 r}-n \omega_{c i}\right)^{2}}{2 k_{z}^{2} v_{t i}}}+\frac{\left(T_{i} / T_{e}\right)^{3 / 2}}{\mu^{1 / 2}}\left(1+\frac{d v_{d e} / d x}{u \omega_{c e}}\right)\left(1-\frac{k_{z} v_{d}}{\omega_{1 r}}\right)=0
$$

Because usually $\frac{d v_{d e} / d x}{u \omega_{c e}}<<1$, meaning that the shear in the electron parallel flow is much less critical at the marginal stability than the shear in the ion parallel flow, the expression of the critical electron drift necessary to destabilize EIC waves in inhomogeneous ion parallel flow plasmas is

$$
v_{d}^{c}=\frac{\omega_{1 r}}{k_{z}}\left\{1+\Gamma_{n}(b) \frac{\mu^{1 / 2}}{\left(T_{i} / T_{e}\right)^{3 / 2}}\left[1-\frac{d v_{d i} / d x}{u \omega_{c i}}\left(1-\frac{n \omega_{c i}}{\omega_{1 r}}\right)\right] e^{-\frac{\left(\omega_{1 r}-n \omega_{c i}\right)^{2}}{2 k_{z}^{2} v_{i i}^{2}}}\right\} .
$$

This is the expression of the critical electron drift of Gavrishchaka et al. [2000] and Ganguli et al. [2002]. In the zero-shear case, $d v_{d i} / d x=0$, the expression of the critical electron drift found by Drummond and Rosenbluth [1962] and by Kindel and Kennel [1971] is retrieved: 


$$
\left(v_{d}^{c}\right)_{0}=\frac{\omega_{1 r}}{k_{z}}\left\{1+\Gamma_{n}(b) \frac{\mu^{1 / 2}}{\left(T_{i} / T_{e}\right)^{3 / 2}} e^{-\frac{\left(\omega-n \omega_{c i}\right)^{2}}{2 k_{z}^{2} v_{t i}^{2}}}\right\} .
$$

The condition $\frac{d v_{d e} / d x}{u \omega_{c e}}<<1$ of the analysis above considers the case when the shear in the electron parallel flow is of the order of the shear in the ion parallel flow, $d v_{d e} / d x \sim d v_{d i} / d x$ or smaller. It is though possible that the shear in the electron flow to be very large, case in which the electron shear term will influence the wave instability. In this dissertation, the electron parallel-velocity shear is considered negligible and the assumption $\frac{d v_{d e} / d x}{u \omega_{c e}} \ll 1$ always applies.

The effect of sheared ion parallel flow on the wave-particle interaction can be seen from the wave growth-rate expression. Its most prominent feature is the inversion of ion cyclotron damping, a process by which waves gain energy from their interaction with the ions. This effect is not obtainable in homogeneous-flow, isotropic, plasma, where ion cyclotron damping prevails.

The simplified dispersion relation is determined from $D_{r}(k, \omega)=0$, where $D_{r}(k, \omega)$ is the real part of (44). Using the approximation $v_{t i}<v_{d}<<v_{t e}$,

$$
\xi_{e}=\frac{\omega_{1 r}-k_{z} v_{d}(x)}{\sqrt{2}\left|k_{z}\right| v_{t e}}<<1 \text { and } \xi_{i}=\frac{\omega_{1 r}-\omega_{c i}}{\sqrt{2}\left|k_{z}\right| v_{t i}}>1
$$

the real part of $Z\left(\xi_{e}\right)$ is expanded in a power series and the real part of $Z\left(\xi_{i}\right)$ is expanded asymptotically,

$$
\begin{aligned}
& \operatorname{Re}\left(Z\left(\xi_{e}\right)\right)=-2 \xi_{e}+\frac{4}{3} \xi_{e}^{2}-\ldots, \text { and } \\
& \operatorname{Re}\left(Z\left(\xi_{i}\right)\right)=-\frac{1}{\xi_{i}}-\frac{1}{2 \xi_{i}^{3}}-\ldots .
\end{aligned}
$$

An expansion of the electron dispersion function and of the ion dispersion function in which the terms containing the shear are retained yields:

$$
D_{r}(\omega, k) \equiv 1-\sum_{n} \Gamma_{n}(b)\left[\frac{\omega_{1 r}}{\omega_{1 r}-n \omega_{c i}}\left[1+\left(\frac{k_{z} v_{t i}}{\omega_{1 r}-n \omega_{c i}}\right)^{2}\right]-\left(\frac{k_{z} v_{t i}}{\omega_{1 r}-n \omega_{c i}}\right)^{2} \frac{d v_{d i} / d x}{u \omega_{c i}}\right]+
$$




$$
\begin{aligned}
& +\left(T_{i} / T_{e}\right)\left[1-\left(\frac{\omega_{1 r}-k_{z} v_{d}}{2^{1 / 2} k_{z} v_{t e}}\right)^{2}\right] \approx \\
& \approx-\sum_{n} \Gamma_{n}(b) \frac{\omega_{1 r}}{\omega_{1 r}-n \omega_{c i}}\left[1-\frac{\omega_{1 r}-n \omega_{c i}}{\omega_{1 r}} \frac{k_{y}}{k_{z}} \frac{d v_{d i}}{\omega_{c i} d x}\right]\left(\frac{k_{z} v_{t i}}{\omega_{1 r}-n \omega_{c i}}\right)^{2}+T_{i} / T_{e} .
\end{aligned}
$$

From $D_{r}(\omega, k)=0$ it follows that for each $n$ term the following applies:

$$
\frac{\omega_{1 r}}{\omega_{1 r}-n \omega_{c i}} \sigma_{n}^{2} \Gamma_{n}(b)=\frac{T_{i}}{T_{e}} \frac{\left(\omega_{1 r}-n \omega_{c i}\right)^{2}}{k_{z}^{2}} \frac{m_{i}}{k_{B} T_{i}} \equiv\left(\frac{\omega_{1 r}-n \omega_{c i}}{k_{z} c_{s}}\right)^{2},
$$

where

$$
\sigma_{n}^{2}=1-\frac{k_{y}}{\omega_{c i} k_{z}} \frac{d v_{d i}}{d x}\left(1-\frac{n \omega_{c i}}{\omega_{1 r}}\right)
$$

is the ion-cyclotron-damping factor and $c_{s}{ }^{2}=k_{B} T_{e} / m_{i}$ is the ion sound speed in the limit $T_{\mathrm{e}}>T_{\mathrm{i}}$. With this notation, the simplified dispersion relation for multi-harmonic ion cyclotron waves in inhomogeneous parallel-ion-flow plasmas is

$$
\omega_{1 r} \approx n \omega_{c i}+\left[n \omega_{c i} k_{z}^{2} c_{s}^{2} \sigma_{c}^{2} \Gamma_{n}\left(k_{y}^{2} \rho_{i}^{2}\right)\right]^{1 / 3} .
$$

A more detailed derivation is presented in Appendix 5.

This relation shows that the departure from the ion gyrofrequency (or its integer multiples) is small because for EIC waves $\omega_{\mathrm{i}}>>k_{\mathrm{z}} c_{\mathrm{s}}$, and both $\sigma_{\mathrm{c}}$ and $\Gamma_{\mathrm{n}}$ are of the order of unity or smaller. Therefore, one can use $\omega_{\mathrm{lr}} \approx n \omega_{c i}$. Also, since the sign of $\sigma_{\mathrm{c}}{ }^{2}$ can be either positive or negative, it means that SMIC wave frequency can be either $\omega_{1 \mathrm{r}}<n \omega_{c i}$ or $\omega_{\mathrm{lr}}>n \omega_{c i}$.

Homogeneous plasma theory [Drummond and Rosenbluth, 1962; Kindel and Kennel, 1971] and experiments [D'Angelo and Motley, 1962; Rassmusen and Schritwieser, 1991] showed that the current-driven EIC wave frequency is slightly above the ion cyclotron frequency,

$$
\omega_{1 r}^{2}=\omega_{c i}^{2}+k_{y}^{2} c_{s}^{2}
$$

Because usually $k_{\mathrm{y}} c_{\mathrm{s}}<<\omega_{c i}$, it follows that $\omega_{1 \mathrm{r}} \approx \omega_{\mathrm{i}}$.

The SMIC wave growth rate was obtained by Ganguli et al. [2002]: 


$$
\gamma \approx \omega_{c i}\left\{\frac{\tau^{3 / 2}}{\mu^{1 / 2}}\left(\frac{k_{z} v_{d}}{\omega_{1 r}}-1\right)-\sum_{n} \Gamma_{n}\left[1-\frac{d v_{d i} / d x}{u \omega_{c i}}\left(1-\frac{n \omega_{c i}}{\omega_{1 r}}\right)\right] e^{-\frac{\left(\omega_{1 r}-n \omega_{c i}\right)^{2}}{2 k_{z}^{2} v_{t i}}}\right\}
$$

The first parentheses, which is positive as long as $k_{z} v_{d}>\omega_{1 r}$, represents the balance between inverse electron Landau damping and electron Landau damping. The bracket represents the ion cyclotron damping term. In order for the wave to grow exponentially, the balance between inverse electron Landau damping and electron Landau damping must overcome ion cyclotron damping.

Several aspects can be highlighted now. First, the growth rate contains the ioncyclotron damping factor (54) that is always unity in the absence of shear. In the presence of shear, the factor can become smaller than unity

$$
0<1-\frac{d v_{d i} / d x}{u \omega_{c i}}\left(1-\frac{n \omega_{c i}}{\omega_{1 r}}\right)<1
$$

which means that ion cyclotron damping can be reduced by the presence of shear. Given the same balance between inverse electron Landau damping and electron Landau damping in the case of reduced ion cyclotron damping, the net wave growth will be enhanced. Furthermore, the shear factor can even switch sign and become negative,

$$
1-\frac{d v_{d i} / d x}{u \omega_{c i}}\left(1-\frac{n \omega_{c i}}{\omega_{1 r}}\right)<0
$$

meaning that ion cyclotron damping becomes inverse ion cyclotron damping, which supports the wave growth rather than damps it. This new growth mechanism, derived in the local limit, does not involve resonant ions, i.e. ions with drift velocity in resonance with waves' parallel phase velocity.

Another significant predicted feature of this mechanism is that ion cyclotron waves can be excited even in the absence of electron drift, $v_{d}=0$. If the factor is negative and large enough (in absolute value) to overcome electron Landau damping, shear-driven ion cyclotron waves can grow spontaneously in inhomogeneous plasmas. Equation (59) expresses the necessary condition for this way of destabilization of ion cyclotron waves.

Higher order harmonics of ion cyclotron waves are also destabilized by the presence of shear in the ion parallel flow. The critical shear necessary to destabilize the $n$-th harmonic is found by setting $v_{d}=0$ in (48) in the strong shear limit, 


$$
\frac{d v_{d i} / d x}{u \omega_{c i}}\left(1-\frac{n \omega_{c i}}{\omega_{1 r}}\right)>>1,
$$

the critical shear is given by

$$
\frac{\left(d v_{d i} / d x\right)_{c r i t}}{\omega_{c i}}=\frac{\left(T_{i} / T_{e}\right)^{3 / 2}}{\mu^{1 / 2}} \frac{u}{\Gamma_{n}(b)} \frac{\exp \left[-\left(\frac{\omega_{1 r}-n \omega_{i}}{2^{1 / 2} k_{z} v_{t i}}\right)^{2}\right]}{1-\frac{n \omega_{c i}}{\omega_{1 r}}} .
$$

Considering that $\omega_{\mathrm{r} r} \sim n \omega_{c i}$ and that the specific harmonic term dominates, using

$$
\begin{aligned}
& D_{r}(\omega, k) \equiv 1-\sum_{n} \Gamma_{n}(b)\left[\frac{\omega_{1 r}}{\omega_{1 r}-n \omega_{c i}}\left[1+\left(\frac{k_{z} v_{t i}}{\omega_{1 r}}\right)^{2}\right]-\left(\frac{k_{z} v_{t i}}{\omega_{1 r}-n \omega_{c i}}\right)^{2} \frac{d v_{d i} / d x}{u \omega_{c i}}\right]+ \\
& +\frac{T_{i}}{T_{e}}\left[1-\left(\frac{\omega_{1 r}-k_{z} v_{d}}{2^{1 / 2} k_{z} v_{t e}}\right)^{2}\right] \approx 1+\frac{T_{i}}{T_{e}}-\Gamma_{0}(b)-2 \sum_{n=1}^{\infty} \Gamma_{n} \frac{\omega_{1 r}{ }^{2}}{\omega_{1 r}{ }^{2}-n^{2} \omega_{c i}^{2}}
\end{aligned}
$$

and setting

$$
D_{r}(\omega k)=0
$$

it follows that

$$
2 \frac{\Gamma_{n}(b) \omega_{1 r}^{2}}{\omega_{1 r}{ }^{2}-n^{2} \omega_{c i}^{2}}=1+T_{i} / T_{e}-\Gamma_{0}
$$

or

$$
\frac{2}{\left(1-\frac{n \omega_{c i}}{\omega_{1 r}}\right)\left(1+\frac{n \omega_{c i}}{\omega_{1 r}}\right)} \approx \frac{1}{1-\frac{n \omega_{c i}}{\omega_{1 r}}}=\frac{1+T_{i} / T_{e}-\Gamma_{0}}{\Gamma_{n}(b)} .
$$

Thus, the critical shear is

$$
\frac{\left(d v_{d i} / d x\right)_{c r i t}}{\omega_{c i}}=\frac{\left(T_{i} / T_{e}\right)^{3 / 2}}{\mu^{1 / 2}} \frac{1+T_{i} / T_{e}-\Gamma_{0}(b)}{\Gamma_{n}(b)} \frac{k_{z}}{k_{y}} .
$$

For $b>>1, \Gamma_{n}(b) \sim(2 \pi b)^{-1 / 2}$ and to the leading order the critical shear is independent of the harmonic number. A large number of harmonics can be then generated by the same shear 
that is enough for destabilizing the fundamental. Computer simulations by Gavrishchaka et al. [2000] and Ganguli et al. [2002] show good agreement with their prediction above. Experimental results presented later on this work document quantitatively the agreement between theory and experiment. This result is in contrast to the homogeneous plasma case, where the critical electron drift, given in (48), required for destabilizing the $n$-th harmonic is much larger than the drift required for destabilizing the fundamental [Drummond and Rosenbluth, 1962; Kindel and Kennel, 1971].

\section{II.B Electrostatic ion acoustic waves modified by shear in the parallel-ion-velocity}

This section of the chapter presents the modifications to the CDEIA magnetized waves dispersion relation due to the presence of shear in the parallel ion flow. The formalism has been developed by Ganguli et al. [1994] and applied by Gavrishchaka et al. [1998, 1999]. The case of temperature-anisotropic plasma $\left(T_{s \perp} / T_{s / /} \neq 1, s=\right.$ species $)$ was discussed theoretically by Spangler [2001] and Spangler et al. [2002].

The dispersion relation of shear-modified ion-acoustic waves is derived in the same manner presented in the section presenting the ion cyclotron waves in the presence of parallelion-velocity shear. But in the case of ion acoustic waves only the $n=0$ terms for electrons and ions are kept since $\omega<<\omega_{c i}<<\omega_{c e}$. This means that the SMIA waves dispersion relation is simply a limiting case of the more general SMIC waves dispersion relation.

The dispersion relation is thus:

$$
\Gamma_{0}(b) F_{i}+\frac{T_{i}}{T_{e}}\left(1+F_{e}\right)+k^{2} \lambda_{d i}^{2}+1=0
$$

where

$$
\begin{aligned}
& F_{i}=\frac{\omega_{1}}{\sqrt{2}\left|k_{z}\right| v_{t z, i}} Z\left(\frac{\omega_{1}}{\sqrt{2}\left|k_{z}\right| v_{t z, i}}\right)\left(1-\frac{k_{y}}{\omega_{c i} k_{z}} \frac{d v_{d i}}{d x}\right)-\frac{k_{y}}{\omega_{c i} k_{z}} \frac{d v_{d i}}{d x} \quad \text { and } \\
& F_{e}=\frac{\left(\omega_{1}-k_{z} v_{d}(x)\right)}{\sqrt{2}\left|k_{z}\right| v_{t z, i}} Z\left(\frac{\omega_{1}-k_{z} v_{d}(x)}{\sqrt{2}\left|k_{z}\right| v_{t z, e}}\right)\left(1+\frac{k_{y}}{k_{z} \omega_{c e}} \frac{d v_{d e}}{d x}\right)+\frac{d v_{d e}}{d x} \frac{k_{y}}{k_{z} \omega_{c e}} .
\end{aligned}
$$


This is the dispersion relation of Gavrishchaka et al [1998]. The anisotropic generalization was derived by Spangler [2001]:

$$
\sum_{n} \Gamma_{n}(b) F_{n i}+\frac{T_{\perp i}}{T_{\| e}}\left(1+F_{o e}\right)+\frac{T_{\perp i}}{T_{\| i}}+k^{2} \lambda_{D i \perp}^{2}=0 .
$$

From (67) a simplified version of the dispersion relation for SMIA waves can be found. The isotropic limit is discussed here. In the long wavelength limit $k_{\perp} \lambda_{d i}<<1$ and denoting

$$
\xi_{e}=\frac{\omega_{1 r}-k_{z} v_{d}(x)}{\sqrt{2}\left|k_{z}\right| v_{t e}} \text { and } \xi_{i}=\frac{\omega_{1 r}}{\sqrt{2}\left|k_{z}\right| v_{t i}},
$$

since $\xi_{e}<1$ and $\xi_{i}>1$, the real part of $Z_{e}$ is expanded in a power series and the real part of $Z_{i}$ is expanded asymptotically,

$$
\begin{aligned}
& \operatorname{Re}\left(Z_{e}\right)=-2 \xi_{e}+\frac{4}{3} \xi_{e}^{2}-\ldots, \text { and } \\
& \operatorname{Re}\left(Z_{i}\right)=-\frac{1}{\xi_{i}}-\frac{1}{2 \xi_{i}^{3}}-\ldots .
\end{aligned}
$$

Substituting these expressions in (70) and keeping only the second order terms in $\xi_{e}$, it follows that

$$
\begin{aligned}
& \Gamma_{0}(b)\left[\left(-1-\frac{1}{2 \xi_{i}^{2}}\right)-\frac{d v_{d i}}{d x} \frac{1}{u \omega_{c i}}\left(1+\left(-1-\frac{1}{2 \xi_{i}^{2}}\right)\right)\right]+ \\
& +\frac{T_{i}}{T_{e}}\left[1-2 \xi_{e}^{2}+\frac{d v_{d e}}{d x} \frac{1}{u \omega_{c e}}\left(1-2 \xi_{e}^{2}\right)\right]+1=0 .
\end{aligned}
$$

In limit of $b=\left(k_{y} \rho_{i}\right)^{2}<<1, \Gamma_{0}(b) \approx 1$. For $v_{t i}<v_{d}<<v_{t e}$, (73) becomes,

$$
\begin{gathered}
\left(\frac{k_{z} v_{t i}}{\omega_{1 r}}\right)^{2}-\frac{d v_{d i}}{d x} \frac{1}{u \omega_{c i}}\left(\frac{k_{z} v_{t i}}{\omega_{1 r}}\right)^{2}-\frac{T_{i}}{T_{e}}\left[1-\left(\frac{k_{z} v_{t e}}{\omega_{1 r}}\right)^{-2}+\right. \\
\left.+\frac{1}{u \omega_{c e}} \frac{d v_{d e}}{d x}\left(1-\left(\frac{k_{z} v_{t e}}{\omega_{1 r}}\right)^{-2}\right)\right]=0 \text { and } \\
\left(k_{z} v_{t i}\right)^{2}\left(1-\frac{d v_{d i}}{d x} \frac{1}{u \omega_{c i}}\right)-\omega_{1 r}{ }^{2}\left(1+\frac{d v_{d e}}{d x} \frac{1}{u \omega_{c e}}\right) \frac{T_{i}}{T_{e}}+\left(\frac{\omega_{1 r}{ }^{2}}{k_{z} v_{t e}}\right)^{2}\left(1+\frac{d v_{d e}}{d x} \frac{1}{u \omega_{c e}}\right) \frac{T_{i}}{T_{e}}=0 .
\end{gathered}
$$


Since the last term in (75) is very small compared with the other two,

$$
\omega_{1 r} \approx k_{z} \sqrt{\frac{k_{B} T_{e}}{m_{i}}} \frac{\sqrt{1-\frac{d v_{d i}}{d x} \frac{1}{u \omega_{c i}}}}{\sqrt{1+\frac{d v_{d e}}{d x} \frac{1}{u \omega_{c e}}}}
$$

Because usually $d v_{\mathrm{de}} / d x\left(u \omega_{\mathrm{ee}}\right)^{-1}<<1,1+\frac{d v_{d e}}{d x} \frac{1}{u \omega_{c i} \mu} \cong 1$, and (76) becomes

$$
\omega_{1 r} \approx k_{z} \sqrt{\frac{k_{B} T_{e}}{m_{i}}} \sqrt{1-\frac{d v_{d i}}{d x} \frac{1}{u \omega_{c i}}}
$$

Equation (77) is the simplified dispersion relation for SMIA waves found by Gavrishchaka et al. [1998]. In the anisotropic case, the form found by Spangler et al. [2002] is

$$
\omega_{1 r} \approx k_{z} \sqrt{\frac{k_{B} T_{e z}}{m_{i}}} \sqrt{1-\frac{d v_{d i}}{d x} \frac{1}{u \omega_{c i}}}
$$

In the case of homogeneous ion parallel flow, $d v_{d i} / d x=0, \sigma=\sqrt{1-\left(u \omega_{c i}\right)^{-1} d v_{d i} / d x} \equiv 1$, which leads to $\omega_{1 r}=k_{z} c_{s}$ which is the ion acoustic waves dispersion relation in homogeneousion-flow plasma derived by Fried and Gould [1961].

Equation (78) shows that for $\left(\omega_{c i} u\right)^{-1} d v_{d i} / d x<0$, a mode with frequency proportional to $k_{z} c_{s}$ can exist. This is the SMIA mode. On the other hand, for $\left(\omega_{c i} u\right)^{-1} d v_{d i} / d x>0$, then $\omega_{\mathrm{lr}}=0$, meaning that a purely growing instability is destabilized in this case. This is the D'Angelo instability [D'Angelo, 1965] which grows in the ion frame and propagates in the laboratory frame with the ion drift velocity $v_{d i}$. D'Angelo [1965] showed that the real and the imaginary parts, respectively, of the wave angular frequency are given by

$$
\omega_{1 r}=0 \text { and } \gamma=k_{z} c_{s}\left(1-\omega_{c i}^{-1} \frac{k_{y}}{k_{z}} \frac{d v_{d i}}{d x}\right)^{1 / 2}
$$


The physical mechanism that allows SMIA waves to grow even in plasmas with equal ion and electron temperatures is the following: the wave parallel phase velocity is upshifted from $c_{s}$ to $\sigma c_{s}(\sigma>1)$ due to the presence of the shear. Thus, for large $\sigma$ it is placed in a smallslope region of the ion velocity distribution and ion Landau damping is greatly reduced. This effect will have ramifications on the wave propagation and wave growth rate.

The factor

$$
\sigma^{2}=1-\frac{k_{y}}{\omega_{c i} k_{z}} \frac{d v_{d i}}{d x}
$$

is simply the $n=0$ approximation of the previously-defined ion-cyclotron-damping factor

$$
\sigma_{n}^{2}=1-\left(1-\frac{n \omega_{c i}}{\omega_{1 r}}\right) \frac{k_{y}}{\omega_{c i} k_{z}} \frac{d v_{d i}}{d x}
$$

another piece of evidence that SMIA waves are just a lower-frequency manifestation of the general (higher-frequency) destabilization mechanism of SMIC waves. There is also another argument supporting this idea: taking the $n=0$ approximation of the SMIC wave simplified dispersion relation (55), in the limit $b<<1, \Gamma_{0}(b)=1$, one obtains

$$
\omega_{1 r}=k_{z} c_{s}\left(1-\frac{k_{y}}{\omega_{c i} k_{z}} \frac{d v_{d i}}{d x}\right)^{1 / 2},
$$

which is the SMIA wave simplified dispersion relation (77).

From the generalized dispersion relation, using the imaginary parts of the plasma dispersion functions for electrons and ions, the SMIA waves growth rate in the presence of electron current can be derived. Defining now

$$
\xi_{i}=\frac{\omega_{1 r}}{\sqrt{2}\left|k_{z}\right| v_{t i}} \quad \text { and } \quad \xi_{e}=\frac{\omega_{1 r}-k_{z} v_{d}}{\sqrt{2}\left|k_{z}\right| v_{t e}}
$$

expressing the imaginary parts of both $Z_{e}$ and $Z_{i}$ as

$$
\operatorname{Im}\left(Z_{i, e}\right)=i \pi^{1 / 2} e^{-\xi^{2}}
$$

after substituting in (81), one obtains the imaginary part of the dielectric tensor 


$$
\begin{aligned}
& D_{i}(\omega, k)=\pi^{1 / 2} \frac{\omega_{1 r}}{\sqrt{2}\left|k_{z}\right| v_{t i}} e^{-\left(\frac{\omega_{1 r}}{\sqrt{2} k_{z} v_{t i}}\right)^{2}}\left(1-\frac{d v_{d i}}{d x} \frac{1}{u \omega_{c i}}\right)+ \\
& +\frac{T_{i}}{T_{e}} \pi^{1 / 2} \frac{\omega_{1 r}-k_{z} v_{d}}{\sqrt{2}\left|k_{z}\right| v_{t e}}\left(1+\frac{d v_{d e}}{d x} \frac{1}{u \omega_{c e}}\right)
\end{aligned}
$$

The real part of the dielectric tensor $D_{r}$ is

$$
\begin{aligned}
D_{r}(\omega, k) & =1+\left[-1-\left(\frac{\omega_{1 r}}{k_{z} v_{t i}}\right)^{-2}\right]\left(1-\frac{d v_{d i}}{d x} \frac{1}{u \omega_{c i}}\right)+ \\
& +\frac{T_{i}}{T_{e}}\left(1+\frac{d v_{d e}}{d x} \frac{1}{u \omega_{c e}}\right)\left[1-2\left(\frac{\omega_{1 r}-k_{z} v_{d}}{\sqrt{2} k_{z} v_{t e}}\right)^{2}\right]
\end{aligned}
$$

and

$$
\frac{\partial D_{r}}{\partial \omega_{1 r}}=2 \frac{\left(k_{z} v_{t i}\right)^{2}}{\omega_{1 r}^{3}} \sigma-2 \frac{T_{i}}{T_{e}} \frac{\omega_{1 r}-k_{z} v_{d}}{\left(k_{z} v_{t e}\right)^{2}}\left(1+\frac{d v_{d e}}{d x} \frac{1}{u \omega_{c e}}\right)
$$

Then, the wave growth rate $\gamma$ can be determined in the approximation $T_{i} / T_{e}<1$

$$
\gamma=-\frac{D_{i}}{\partial D_{r} / \partial \omega_{1 r}} \approx\left(\frac{\pi}{8}\right)^{1 / 2} \frac{\omega_{1 r}}{\sigma^{2}}\left(\frac{\omega_{1 r}}{\left|k_{z}\right| v_{t i}}\right)^{3}\left[\frac{\left(T_{i} / T_{e}\right)^{3 / 2}}{\mu^{1 / 2}}\left(\frac{k_{z} v_{d}}{\omega}-1\right)-\sigma^{2} e^{-\left(\frac{\omega_{1 r}}{\sqrt{2} k_{z} v_{t i}}\right)^{2}}\right]
$$

This is the growth rate obtained by Gavrishchaka et al. [1998]. There are two major remarks based on this growth rate expression:

1. The critical electron- $\mathrm{drift} v_{d}^{c}$ (found by setting $\gamma=0$ ) is

$$
v_{d}^{c}=v_{t i}\left(\frac{\sigma}{\left(T_{i} / T_{e}\right)^{1 / 2}}+\frac{\sigma^{3}}{\left(T_{i} / T_{e}\right)^{2}} \mu^{1 / 2} e^{-\frac{\sigma^{2}}{2\left(T_{i} / T_{e}\right)}}\right),
$$

If $\sigma=1$, the expression of the critical electron current necessary to destabilize ion acoustic waves in homogeneous-ion-flow plasmas [Kindel and Kennel, 1971] is retrieved,

$$
\left(v_{d}^{c}\right)_{C D E I A}=v_{t i}\left(\frac{1}{\left(T_{i} / T_{e}\right)^{1 / 2}}+\frac{\mu^{1 / 2}}{\left(T_{i} / T_{e}\right)^{2}} e^{-\frac{1}{2\left(T_{i} / T_{e}\right)}}\right)
$$


Gavrishchaka et al. [1998] showed that by minimizing $v_{\mathrm{d}}{ }^{\mathrm{c}}$ with respect to $\sigma$ in order to obtain $\sigma_{\mathrm{m}}$ which satisfies

$$
1+\frac{\mu^{1 / 2} \sigma_{m}^{2}}{\left(T_{i} / T_{e}\right)^{3 / 2}} \exp \left(-\frac{\sigma_{m}^{2}}{2 T_{i} / T_{e}}\right)\left(3-\frac{T_{e} \sigma_{m}^{2}}{T_{i}}\right)=0,
$$

and then by substituting $\sigma_{\mathrm{m}}$ into (89), the minimum critical electron drift in the presence of shear is found, and it is less than that for the homogeneous case [Gavrishchaka et al., 1998]. This behavior is due to the increase in the $\sigma_{\mathrm{m}}>1$ with increasing $T_{\mathrm{i}} / T_{\mathrm{e}}$.

2. For large enough ion parallel-velocity shear $d v_{d i} / d x$, it is possible that $\gamma>0$ even if $v_{d}=0$. This means that wave destabilization is possible even in the case that no electron current is present. The full analysis of this "shear-driven ion acoustic mode" is presented by Gavrishchaka et al. [1999]. Taking into account the $n= \pm 1$ terms in the full dispersion relation (44), they predicted that the critical shear $\left(\omega_{c i} u\right)^{-1} d v_{d i} / d x$ is linearly dependent on $T_{\mathrm{i}} / T_{\mathrm{e}}$ and that shear-driven ion acoustic waves would have a spectrum range of $0.5<\omega / \omega_{c i}<1$ and very large propagation angles $u \rightarrow 0$. 


\section{Chapter III. Experimental device}

This chapter describes the operation of the experimental device. Particular aspects of the device and of the plasma produced and contained in it, directly relevant to the experiments presented in this work, are highlighted.

\section{III.A Experimental device characteristics}

The experiments are performed in the West Virginia University Q machine (WVU-Q). The machine, described in detail in the dissertation of Carroll [1997], was used to benchmark the experimental verification of the Inhomogeneous Energy Density-Driven Instability (IEDDI) mechanism. It is a significant upgrade of the original WVU Q machine $\left(\mathrm{Q}_{0}\right)$, described in the thesis of Cerkovnik [1989] and in the dissertation of Amatucci [1994]. The work of the latter documented the operation of the segmented disk electrode and described the first experimental evidence of the IEDDI mechanism [Ganguli et al., 1989].

The main components of the Q-machine, shown in Fig. 1, are the vacuum chamber including its pumps, the plasma source including the power supplies, and the magnetic field power supply and its set of electromagnets. The diagnostic equipment and instrumentation are described in chapter IV.

\section{III.A.1 Vacuum chamber}

The purpose of the vacuum chamber is to contain the plasma in a reduced-pressure environment (thus minimizing the effect of collisions between plasma particles and neutral background particles). The chamber is a $25-\mathrm{cm}$ outer diameter stainless steel vessel $4 \mathrm{~m}$ in length. Of the 56 ports mounted in groups of four at 14 axial positions (spaced $26.5 \mathrm{~cm}$ apart and mounted at $90^{\circ}$ azimuthal increments), 48 are accessible for plasma diagnostics. The six outermost available bottom ports at each end of the chamber connect it to the pumping station via a six-arm vacuum station manifold.

The main pumping stations each consist of a Pfeiffer TMU 520 turbomolecular pump which evacuates the chamber and a Pfeiffer MD 4T diaphragm pump which serves both as a fore pump for the TMU 520 and a roughing pump for the vacuum chamber. A Pfeiffer TCP 
380 controller provides push-button operation of each turbo pump and diaphragm pump pair during the starting, regular and shut-down phases. Two secondary pumping stations are each mounted via an L-shaped tube to the hot plate assembly at the end of the vacuum chamber. The secondary pumps evacuate the hot plate assembly and inhibit arc-discharges from occurring during elevated-neutral-pressure operations and during the process of warming-up the hot plate. These stations each consist of a Pfeiffer TMU 170 turbomolecular pump and a Pfeiffer MD 3T diaphragm pump, controlled via a Pfeiffer TCP 350 controller.

The heat radiated by the plasma sources is dissipated by a cryopanel at each plasma source. The dissipation keeps the chamber walls cool enough to absorb neutrals (not confined by the magnetic field) and thus maintain a low neutral-pressure. In the cryopanel cavity formed by the two cylindrical metal sheets $(25-\mathrm{cm}$ long, $20-\mathrm{cm}$ outer-shell diameter and 19$\mathrm{cm}$ inner-shell diameter, respectively) circulates a mixture of water and glycol from the FTS chilled-water recirculator. The mixture, introduced through 4 inlet tubes, flows along a pattern of walls (welded between the two sheets) forming a grooved assembly which assures a steady, unidirectional flow, and exits through 4 outlet tubes.

\section{III.A.2 Plasma source}

The plasma is produced on the surface of the hot plate by thermionic emission of electrons and by contact ionization of an alkali metal atomic beam. Two thoriated tungsten filaments, each supported by the same two tungsten rods, are located within the hot plate cavity and are used to heat the molybdenum cathode, also located within the hot plate cavity, via electron bombardment. Electron emission from the heated cathode is used to heat the hot plate via a second stage of electron bombardment. The plasma-source power is provided by a Spellman DC filament supply $(75 \mathrm{~A}, 15 \mathrm{~V})$ typically operated at $28 \mathrm{~A}$ and $12 \mathrm{~V}$. The filaments are biased at $-1.1 \mathrm{kV}$ with respect to the cathode using a Spellman high-voltage power supply $(2 \mathrm{~A}, 1.5 \mathrm{kV})$. During the starting of the ionizer, a weak magnetic field $(0.5 \mathrm{kG})$ is applied in order to direct the electrons accelerated from the filaments to the cathode. A major advantage of this design is that the filaments, the components most vulnerable to failure, are only operated at much smaller magnetic fields for a small fraction of the Qmachine run (typically 20-30 minutes) instead of the entire run (between 7-10 hours). This 
duty cycle minimizes the $\boldsymbol{J} \times \boldsymbol{B}$ forces acting on the filament that can considerably shorten its lifetime.

The disk-cathode is electron-beam welded to a tantalum support tube, that is in turn spot-welded to a stainless steel mounting collar. The grooved face of the cathode is filled with a lanthanum hexaboride paste which is a very good electron emitter. Thus, the electron flux towards the hot plate is enhanced. The cathode begins to emit electrons around $1700^{\circ} \mathrm{C}$. This temperature is achieved by the $400 \mathrm{~W}$ electron-beam emitted by the filaments $(0.4 \mathrm{~A}$ at 1.0 $\mathrm{kV})$. The cathode bias with respect to the hot plate is gradually set to $-1.5 \mathrm{kV}$ using a Spellman SR6 high-voltage power supply (total power $6 \mathrm{~kW}$ ) as the electron emission between the cathode and the hot plate increases. Self-sustained operation mode is attained when the radiated power from the hot plate is sufficient to keep the cathode hot and emissive, which typically occurs when $1.5 \mathrm{~kW}(1 \mathrm{~A}$ at $1.5 \mathrm{kV})$ is provided to the hot plate by the cathode. At this point, the filament-cathode power supplies can be turned off. Normally, a (2.5-3) $\mathrm{kW}$ hot plate radiated power is maintained throughout the Q-machine run.

The hot plate uniform temperature profile is achieved by using a disk-cathode with $4.75 \mathrm{~cm}$ diameter while the hot plate diameter is $6.4 \mathrm{~cm}$. The hot plate is electrically insulated from the chamber by a ceramic ring and ceramic washers that serve to maintain the partial vacuum seal. The BOS/S 100-4 bipolar power supply (one for each plasma source) biases the hot plate at voltages in the range of from $-100 \mathrm{~V}$ to $+100 \mathrm{~V}$ for currents up to $4 \mathrm{~A}$ (the usual cathode-hot plate discharge current is 1.8-2.5 A).

Rhenium is electro-deposited on the tungsten surface of the hot plate to increase the ionization of alkali metal, here barium, used in these experiments. Some 100 monolayers were coated, and several textual patches are visible on the rhenium coating.

Another part of the plasma source is represented by the two atomic beam ovens (one at each plasma source). The ovens, inserted completely within the vacuum chamber to prevent heat radiative losses at atmospheric pressure, consist of an annular effuser, two bodies and a base. The effuser is heated by the hot plate because it is mounted cylindrically symmetric around it at 1-cm distance. To ensure effusers' uniform heating, the effusers are fitted with heat shields that extend axially to partly cover the hot plate.

The bodies (15-cm long, 2-cm diameter tubes) of the ovens are wrapped with 2.0-mm diameter Thermocoax through which a current providing 120-140 W (3-4A at 30-35 V, from a Sorensen dc power supply) heats the solid barium chips inside the bodies. The tubes are 
mounted at $30^{\circ}$ downwards so that as the barium sublimates and the vapors reach the effuser, the molten material remains pooled away from the effuser.

\section{III.B Magnetic field}

Plasma radial confinement is achieved with a uniform axial magnetic field produced by 41 magnets $(33 \mathrm{~cm}$ inner diameter) grouped in sets of three (except the end groups which have four magnets each). The 11 groups inboard have two magnets slidable around a central fixed one. The two groups outboard have two magnets on the outside of the fixed magnet to compensate the end effects of the finite-length solenoid. Magnetic field strength is currentcontrolled using the current-control dial of a Walker Power power-supply (1000A at $250 \mathrm{~V})$. The highest attainable magnetic field is $\mathrm{B}=3 \mathrm{kG}(=0.3 \mathrm{~T})$ for the $0.8 \Omega$ magnetic set. Its direction can be pointing either toward the north plasma source or toward the south plasma source. The direction change is made by switching the leads at the output of the Walker Power power-supply that go to the magnetic set.

Throughout the experiments presented here, all magnets are arranged in the compact configuration, the sliding magnets being pressed against the fixed magnets. This configuration is used to enable easy access to the diagnostic ports, especially to those where the laser induced fluorescence apparatus is mounted. Fig. 2 shows the axial magnetic field for the compact configuration. The maximum ripple (peak-to-peak magnetic field strength variation) is of the order of $\delta B / B \sim 6.5 \%$ over an axial distance $\delta z=26.5 \mathrm{~cm}$. The effect of the ripple can consist of creating plasma-diameter rippling of the order of the magnetic field ripple and of affecting the local value of some parameters that depend on the magnetic field strength, such as the particle angular gyrofrequency.

The magnets are connected in series using welding cable and are cooled by water. Magnet support and precise $x-y$ positioning are provided by ground stainless steel rods along which the magnetic coils can be slid for precise axial positioning. 


\section{III.C Plasma characteristics}

The plasma used in the experiments presented in this dissertation consists of electrons and barium ions. Barium vapors (melting point $725^{\circ} \mathrm{C}$ ) experience contact ionization on the rhenium-coated hot plate surface. Ionization occurs due to the potential difference between the work function of rhenium $\left(W_{f}=4.96 \mathrm{eV}\right)$ and the ionization potential of barium $\left(W_{i} / e=5.19\right.$ V). Saha's equation

$$
\frac{n_{i}^{2}}{n_{n}}=\frac{g_{i}}{g_{n}}\left(\frac{2 \pi m k_{B} T}{h^{2}}\right)^{3 / 2} \exp \left(-\frac{W_{i}-W_{f}}{k_{B} T}\right),
$$

where $n_{i}$ is the density of ions and $n_{n}$ is the density of neutrals (atoms) and $g_{i}$ and $g_{n}$ are the statistical weights of the ions and of the neutrals, respectively, shows that for a hot plate temperature of $T=2000 \mathrm{~K}$, the ionization probability is around $20 \%$. The ionization degree in the Q-machine plasma column is as high as $99 \%$, since the neutrals cannot be confined by the applied magnetic field and are lost due to their recombination at the cold chamber walls. The same equation can predict the number of ions in an excited state, if $W_{i}$ and $W_{f}$ are replaced by the energy of the excited state and the energy of the ground (fundamental) state, respectively. Of a particular interest to these experiments is the number of ions in the ${ }^{5} \mathrm{D}_{5 / 2}$ excited state, since these ions will be laser-stimulated in the process of using the laser induced fluorescence (LIF) technique for acquiring information on the ions' velocity distribution. At a hot plate temperature of $T=2000 \mathrm{~K}$, the proportion of ions in the $5{ }^{2} \mathrm{D}_{3 / 2}$ excited state is approximately $12 \%$ of the entire ion population. At lower temperatures this number reduces significantly, making the use of LIF measurements more challenging. Plasma created in this manner has the form of a column, $3-\mathrm{m}$ long and $6.5 \mathrm{~cm}$ in diameter. A summary of barium plasma parameters ranges is provided in Table 1. A typical barium-plasma current-voltage characteristic from a Langmuir probe is shown in Fig. 3. 


\section{Chapter IV. Diagnostics}

This chapter presents the diagnostics used in the experiments and outlines the methods used for data acquisition and analysis.

\section{A Electrostatic probes}

Electrostatic (Langmuir) probes are electrodes inserted in the plasma. The currentvoltage characteristic of the probe is used for determining some of the plasma parameters. The characteristic is obtained by applying a swept voltage to the probe and measuring the corresponding current collected from the plasma. The floating potential, the plasma potential, the plasma density, and the electron temperature can be determined with this type of probe. A typical current-voltage characteristic is shown in Fig. 3. For very negative voltages applied, here below $-8 \mathrm{~V}$, only ions will be collected, giving thus the ion saturation regime of the current-voltage dependence. For very positive voltages, here above $-2 \mathrm{~V}$, only electrons will be collected by the probe tip; thus, the electron saturation regime is obtained. Due to the larger mobility, more electrons will reach the probe in the electron-saturation regime than ions in the ion-saturation regime; thus $\left|I_{e \text { sat }}\right|>I_{\text {isat }}$.

For a probe immersed in an unmagnetized plasma, $I_{e}$ sat at the plasma potential and $I_{i \text { sat }}$ are

$$
\left|I_{\text {esat }}\right|=\frac{n e A_{s}}{4} \sqrt{\frac{8 k_{B} T_{e}}{\pi m_{e}}} \text { and } I_{\text {isat }}=0.61 n e A_{s} \sqrt{\frac{k_{B} T_{e}}{m_{i}}}
$$

respectively. Here, $n$ is plasma density and $A$ is the surface of the area of the sheath surface surrounding the probe tip where the current is collected. The coefficient $0.61\left(=2.718^{-1 / 2}\right)$ comes from the calculation of the plasma density at the edge of the sheath.

The floating potential $V_{\mathrm{f}}$ is the potential of the probe corresponding to zero net current collected by the probe tip. The plasma potential $V_{\mathrm{p}}$ is the value of the potential for which $d I / d V=0$. To determine the electron temperature, the exponential regime, which is the currentvoltage regime situated between the ion saturation and the electron saturation regimes, must be used. This is so because for probe voltages around the floating voltage the current collected by the probe is neither electron nor ion dominated; thus a dynamical equilibrium between the two species is created within a sheath region of a few Debye lengths around the probe tip. 
Here, the collected electron current varies exponentially with the applied voltage in the case of a Maxwellian plasma distribution:

$$
I_{e}(V)=I(V)-I_{\text {isat }}=I_{e s a t} \exp \left(\frac{e\left(V-V_{p}\right)}{k_{B} T_{e}}\right) .
$$

where $V<V_{\mathrm{p}}$. Using a linear fit analysis, the electron temperature is

$$
T_{e}=\frac{e}{k_{B}} \frac{d V}{d\left(\ln I_{e}\right)} \text {. }
$$

An important correction to this formula must be made when the electron drift speed $v_{d e}$ is large, i.e. becomes comparable to the electron thermal speed $v_{t e}=\left(k_{\mathrm{B}} T_{e} / m_{e}\right)^{1 / 2}$. As shown by Sheridan and Goree [1994], for $v_{d e} \geq 0.2 v_{t e}$, the electron temperature is overestimated by the above formula because the collected current is modified by the drifting electrons. In this case, $I_{e}(V)$ becomes:

$$
\begin{aligned}
I_{e}\left(V_{r}\right)=I_{\text {esat }} & \int_{0}^{2 \pi} \frac{d \theta}{2 \pi}\left[\exp \left\{-\left[\left(\frac{-e V_{r}}{k_{B} T_{e}}\right)^{1 / 2}-\frac{v_{d e}}{v_{t e}} \cos \theta\right]^{2}\right\}+\right. \\
& \left.+\sqrt{\pi} \frac{v_{d e}}{v_{t e}} \cos \theta \times \operatorname{erfc}\left[\left(\frac{e V_{r}}{k_{B} T_{e}}\right)^{1 / 2}-\frac{v_{d e}}{v_{t e}} \cos \theta\right]\right]
\end{aligned}
$$

where $V_{\mathrm{r}}=V-V_{\mathrm{p}}$. The electron temperature will be then:

$$
T_{e}=\frac{e}{k_{B}} \frac{d V}{d\left(\ln I_{e}\right)}\left[\frac{I_{\text {esat }}}{I_{e}} \frac{d\left(I_{e} / I_{e s a t}\right)}{d\left(e\left(V-V_{p}\right) / k_{B} T_{e}\right)}\right] . .
$$

No electron drift speeds larger than $0.15 v_{\text {te }}$ were detected in the experiments presented in this dissertation.

Using the above expression for $I_{i \text { sat }}$, the plasma density $n$ is given by:

$$
n=\frac{I_{\text {isat }}}{0.61 e A_{s}} \sqrt{\frac{m_{i}}{k_{B} T_{e}}} .
$$

The sheath area $A_{\mathrm{s}}$ and the collection area $A_{\mathrm{p}}$ of a cylindrical probe are related by the formula

$$
A_{\mathrm{s}}=A_{\mathrm{p}}\left(1+r_{\mathrm{s}} / r_{\mathrm{p}}\right)
$$

where $r_{\mathrm{s}}$ is the sheath thickness and $r_{\mathrm{p}}$ is the probe radius [Hutchinson, 1987]. 
This relation between the sheath area and the collection area of a cylindrical probe is used in the experiments presented in this dissertation because the exposed tips of the Langmuir probes used in the experiments are small cylindrical shells. This "halo" type probetip [Koepke et al., 1999] is separated from the rest of the probe stem by a distance comparable to its length. The dimensions of the shell (diameter $2 r_{\mathrm{p}}=2 \mathrm{~mm}$, length $l=1 \mathrm{~mm}$ and thickness $0.2 \mathrm{~mm}$ ) are small compared to the plasma dimensions. These dimensions where used such that to maximize the current collected but to avoid as much as possible perturbing the plasma. Thus, for this type of probe, $A_{\mathrm{p}}=2 \pi r_{\mathrm{p}} l$. The sheath thickness is a few Debye lengths at the floating potential [Hutchinson, 1987], thus using the values for the Debye lengths given in Table 1, one obtains $r_{\mathrm{s}} \propto 6 \lambda_{\mathrm{Di}} \approx 0.03 \mathrm{~mm}$. Therefore, the area of the sheath surface is almost equal to the collection area of the probe, $A_{\mathrm{s}}=1.03 A_{\mathrm{p}}$.

Plasmas used in the experiments described in this work are magnetized. The main effect of the magnetic field is to cause the ions and electrons to move no longer in straight lines (as in unmagnetized plasmas) but to orbit around the field lines while drifting along the field lines. The importance of the magnetic field effects is determined by the ratio $\rho / r_{\mathrm{p}}$, where $\rho$ is the species gyroradius [Hutchinson, 1987]. For the magnetic field values listed in Table 1, the following relations apply: $\rho_{\mathrm{e}} / r_{\mathrm{p}} \propto 0.03$ and $\rho_{\mathrm{i}} / r_{p} \propto 3$. This means that in a magnetized plasma, the electron saturation current is decreased since the electron flow towards the probe is impeded. This is caused by the fact that $\rho_{\mathrm{e}} / r_{\mathrm{p}}<1$, i.e., only electrons within a gyrodiameterwide flux tube can reach the probe. Practically, this effect is as if the electron-collection area becomes smaller since electrons can only reach it along the direction perpendicular to the collection surface. Thus, for electrons, $A_{\mathrm{p}}$ becomes the projection of the probe surface in the direction of the magnetic field, for example $2 r_{\mathrm{p}} l$ instead of $2 \pi r_{\mathrm{p}} l$ for a cylinder. If the probe is significantly negative so that most electrons are reflected the analysis of the exponential regime will provide accurately the electron temperature. The ions are relatively unaffected by the magnetic field, since $\rho_{\mathrm{i}} / r_{\mathrm{p}}>1$, thus the ion saturation current is the same as in an unmagnetized plasma [Hutchinson, 1987].

A computer routine (written in ASYST - see Amatucci, [1994]) is used to determine the current-voltage characteristic of the probe. The routine assigns to each of the 512 data points in the array a voltage (user-adjustable between $-100 \mathrm{~V}$ and $100 \mathrm{~V}$ ). The computer then sends the voltage-sweep command to a Keithly 2400 Source meter, which applies the voltage 
to the probe and records the current collected. The data are sent to the computer and plotted as $I(V)$ vs. $V$ (see Fig.3).

Another ASYST routine is used in order to obtain the radial profile of the plasma density. The electrostatic probe is moved radially in increments of $1 \mathrm{~mm}$, with the aid of a computer-controlled Velmex-motor-translation stage, across the entire plasma-column diameter. At each point, the Keithly 2400 Source meter applies $-5 \mathrm{~V}$ (with respect to $V_{f}$, the floating potential) to the probe tip and measures $I_{i \text { sat }}$, thus determining $I_{i \text { sat }}$ as a function of the radial position $r$. The plasma density is then calculated and the result is plotted in the form $n(r)$ vs. $r$, as shown in Fig. 4.

One of the objectives of the work presented in this dissertation is measuring low frequency plasma fluctuations $\delta n=n(t)-n_{0}, \delta V=V(t)-V_{0}$ and $\delta T_{e}=T_{e}(t)-T_{e 0}$. These fluctuations are correlated with the presence of plasma instabilities. Assuming small perturbations in the electron temperature, the plasma density and potential fluctuations can be found as:

$$
\delta n \propto \frac{\delta I_{i, s a t}}{\sqrt{T_{e 0}}} \text { and } \delta V_{p}=\delta V_{f} .
$$

An ASYST routine is set up in order to determine $\delta n$ as a function of the position across the plasma. By DC coupling the probe to the LeCroy 6810 waveform recorder, any fluctuation of $I_{i ~ s a t}$ is recorded as an AC signal superimposed on the user-selected DC offset used (on the order of $-5 \mathrm{~V}$ with respect to $V_{\mathrm{f}}$ ), at each radial position (in increments of $1 \mathrm{~mm}$ to ensure good radial resolution). Thus, the root-mean-squared value of $\delta n(t)$ can be determined. Knowing both $(\delta n)_{\mathrm{rms}}$ and $n_{0}$ at a given location, the quantity $(\delta n)_{\mathrm{rms}} / n_{0}$, the wave amplitude, can be calculated. A typical radial profile of the plasma density fluctuations when the D'Angelo instability [D'Angelo, 1965] is present is shown in Fig. 5.

\section{IV.A.1 Signal analysis from multiple, spaced, probe tips}

The two-tip probes are two independent electrostatic probes, mounted on the same probe shaft, but having separate electric connections/circuits with the signal analyzing devices. Thus, there are two signals provided by this type of probes. The main use of the twotip probe is in determining the wave characteristics, namely the wave frequency spectrum and 
the wavevector components. The probe tips consist of small (0.2-0.3 mm diameter) tungsten spheres. The distance between them is set to be smaller than the wavelengths expected to be measured and is $\delta=0.35 \mathrm{~cm}$ in these experiments. Biasing the probe below the floating potential, the ion saturation current fluctuations can be measured. The ion saturation current fluctuations collected with the electrostatic probes provide information on the spectral components of the studied wave. Using the LeCroy 9424 E digital storage oscilloscope (for real-time monitoring) or the waveform recorder and a computer code that determines the Fast Fourier Transform of the signal time series, the wave spectrum (FFT amplitude as a function of frequency) is obtained. Each probe provides its FFT based on the time series of the signal picked-up. For a time series $a(x, t)$ - meaning the time $t$ dependence of the signal (detected at position $x$ ) amplitude $a$ - the FFT is given by

$$
f(x, \omega)=\sum_{n=-\infty}^{\infty} \int_{-\infty}^{\infty} \alpha_{n} a(x, t) \cos \left(\omega_{n} t\right) d t+i \sum_{n=-\infty}^{\infty} \int_{-\infty}^{\infty} \beta_{n} a(x, t) \sin \left(\omega_{n} t\right) d t .
$$

The FFT amplitude, or the spectral power density, is

$$
S(x, \omega)=<f^{*}(x, \omega) f(x, \omega)>.
$$

In the case of the simultaneous determination of the same signal at different locations, $a\left(x_{1}, t\right)$ and $a\left(x_{2}, t\right)$, respectively, the spectral power density will be a function of the phase difference introduced by the spatial separation $\Delta x$ between the locations where the signal is measured $\Delta x=x_{1}-x_{2}[$ Priestley, 1989]:

$$
S_{12}(\Delta x, \omega)=<f^{*}\left(x_{1}, \omega\right) f\left(x_{2}, \omega\right)>
$$

This is a complex quantity, whose modulus $\left|S_{12}(\Delta x, \omega)\right|$ quantifies the coupling of the signals and whose phase $\Phi_{12}=\arg S_{12}(\Delta x, \omega)$ indicates the phase difference between the two signals: $\Delta \Phi=\Phi_{12}$. The average wavenumber along $\Delta x$ is then $k=(\Delta \Phi / \Delta x)$. For $N$ ensembles of time series, the averaged spectral power density $S_{l}(k, \omega)$ is given by

$$
S_{l}(k, \omega)=\frac{1}{N} \sum_{i=1}^{N} I_{\Delta k}\left(k-k_{i}(\omega)\right) S_{12}^{(i)}(\Delta x, \omega)
$$


In this expression, $I_{\Delta \mathrm{k}}\left(k-k_{i}(\omega)\right)$ is the indicator function which has the values 1 for $-\Delta k / 2<k$ $k_{i}(\omega)<\Delta k / 2$ and 0 elsewhere and $S^{(i)}{ }_{12}$ is the mean value of the spectral density for the $i$-th ensemble of the time series [Priestley, 1989; Kline, 2002].

In order to obtain information regarding the wave propagation characteristics, the components of the wavevector $\boldsymbol{k}$ must be determined. Using the two-tip probe shown in Fig. 6 , the axial $k_{\mathrm{z}}$ and the azimuthal $k_{\theta}$ ( $k_{\mathrm{y}}$ in Cartesian coordinates) components are measured directly. This is accomplished by positioning the probe shaft perpendicular to the magnetic field lines. The two tips are placed at the same radial position $r$, by a radially aligned probe shaft. By rotating the probe shaft around its symmetry axis, the angle made by the line connecting the two tips and the magnetic field lines will vary between $0^{0}$ when the two tips lie of the same field line, $\pm 90^{\circ}$ when the two tips lie on field lines $3.5 \mathrm{~mm}$ apart, and $180^{\circ}$, when the two tips again lie on the same magnetic field line.

When the line is at $0^{\circ}$ or $180^{\circ}$ with respect to the $\boldsymbol{B}$ lines, the probe determines the axial component ( $k_{z}$ or $-k_{z}$, respectively) of the wavevector. Positioning the probe array parallel to the magnetic field line such that the magnetic field points from probe tip 2 towards probe tip 1, if $\Delta \Phi=\Phi_{2}-\Phi_{1}>0$, then the measured wavevector component is $k_{z}>0$. With the same probe array orientation, if $\Delta \Phi<0$, then the measured wavevector component is $k_{z}<0$. When the line is at $90^{\circ}$ or $270^{\circ}$, the azimuthal component $\left(k_{\theta}\right.$ or $-k_{\theta}$, respectively, in a cylindrical coordinate system, and $k_{\mathrm{y}}$ or $-k_{\mathrm{y}}$, respectively, in a Cartesian system) is measured. Generally, if the line is at an angle $\theta$ with respect to $\boldsymbol{B}$, then $k_{y}=k \sin \theta$ and $k_{z}=k \cos \theta$. Practically, the measurements performed in the experiments described later on are based on an ASYST routine which controls (via a GPIB/IEEE-488 interface) a Velmex-motor-driven rotational stage; the probe mounted on the stage is rotated in increments of $18^{\circ}$, until $360^{\circ}$ are completed. At each probe array position, a set of $N$ time series is collected by each probe. Usually, $N=3$, but as many as 10 measurements were taken. Thus, a total of 120 time series are recorded in order to better determine $\boldsymbol{k}$ when $N=3$. After the acquisition of the time series, a MATLAB routine (shown in Appendix 3) calculates the cross-correlated phase difference between the two time series acquired at a given angle by averaging in a narrow frequency range. The width of this frequency range is chosen by the user based on the spectral position of the cross-correlated power such that it is not larger than the frequency range where the FFT power is located. The phase difference (at each angle, for each realization) is then plotted 
against $\theta$. By fitting a sinusoid to the obtained curve using the method of chi-square fitting, the two components $k_{y}$ and $k_{z}$ of $\boldsymbol{k}$ are determined as described above.

For data presented in Fig. 7, the vertical error bars (indicated by the vertical size of the symbols) represent the uncertainty in determining the phase difference at the corresponding probe angle for the corresponding realization based on the phase difference variation within the interrogation frequency range. The fitted sinusoid lies within the error bars for most of the data points and yields $k_{z}=-0.033 \mathrm{~cm}^{-1}$ and $k_{y}=-0.726 \mathrm{~cm}^{-1}$.

There are a number of issues that must be carefully accounted for when using this method. When determining a very small $k_{z}$, i.e., a wavelength component which is much larger than the probe-tip separation, at the angle $\theta=0^{0}$ the phase difference obtained with this probe (having the tip distance $\delta$ small) will also be small. Any uncertainty in the phase difference $\Delta \Phi_{\mathrm{z}}=k_{\mathrm{z}} \delta$ at $\theta=0$ leads to uncertainties in $k_{\mathrm{z}}$. To overcome this problem, measurements can be acquired using a probe array with a larger $\delta$ (since the large axial wavelength can accommodate large $\delta$ ). After the phase difference as a function of $\theta$ is plotted, the fitted sinusoidal curve can be graphically adjusted to estimate the uncertainty in $k_{z}$ based upon the rest of the data points (except at $\theta=0^{0}$ and $\theta=360^{\circ}$ ). Using this technique it has been found that values as small as $k_{z}=0.015 \mathrm{~cm}^{-1}$ can be accurately measured. When there exists multiple spectral features, such as when many eigenmodes of an instability are present [Carroll, 1997], the width of the investigation frequency range must be decreased so that each determination of $k_{\mathrm{z}}$ corresponds to a specific spectral feature. Using the $k$-spectrum to determine the components of the wavevector works well and is especially used in the case of multiple eigenmodes. The main advantage is displaying which values of $k$ are associated with large amplitude fluctuations just as the frequency spectrum displays which values of the frequency are associated with large amplitude fluctuations. Such $k$-spectra obtained when the two-tip probe is positioned along or perpendicular to the $\boldsymbol{B}$ lines resolve the various eigenmodes according to their axial and azimuthal propagation characteristics [Carroll, 1997].

Fig. 8 presents the $k_{\mathrm{y}}$ spectrum $\left(\theta=270^{\circ}\right)$ for data shown in Fig. 7. Here, the two largest $k$-spectrum components have $\left|k_{\mathrm{y} 1}\right|=\left|\Delta \Phi_{1}\right| / \delta=(0.24 \quad \mathrm{rad}) /(0.35 \quad \mathrm{~cm})=0.69 \quad \mathrm{~cm}^{-1} \quad$ and $\left|k_{\mathrm{y} 2}\right|=\left|\Delta \Phi_{2}\right| / \delta=(0.25 \mathrm{rad}) /(0.35 \mathrm{~cm})=0.72 \mathrm{~cm}^{-1}$, respectively. The wave frequency is here $\omega / 2 \pi=25.7 \mathrm{kHz}$. The sign of the wavevector component is determined from the sign of the phase difference $\Delta \Phi$. 
Aliasing is another issue that must be accounted for. For wavevector measurements using two probe tips with fixed $\delta$, spatial aliasing occurs when the wavelength becomes smaller than the distance between the two tips. In power spectrum measurements at a given spatial location, aliasing occurs when the signal's frequency is larger than the so-called Nyquist frequency, defined as $f_{\text {Nyquist }}=($ sample rate/2) of the data acquisition device. In the FFT of an aliased time series measurement, the power spectrum will appear at lower frequencies and spurious peaks at harmonics of the artificially lowered frequency can also appear. Spatial aliasing can be detected by changing $\delta$, since the peaks will be shifted in phase with a non-sinusoidal dependence on $\delta$. The probe tip distance was not changed in the experiments, but the probes were rotated so the effective distance in the parallel and perpendicular directions with respect to the magnetic field was changed. The frequency aliasing can be observed by changing the sampling rate because the aliased peaks will shift in frequency. There was no aliasing seen for the waves detected and analyzed in these experiments. The sample size used in the measurements is either 2048 samples or 4096 samples and the sample rate is varied between $100 \mathrm{ksamples} / \mathrm{s}$ to $1000 \mathrm{ksamples} / \mathrm{s}$, thus yielding a Nyquist frequency range of $50 \mathrm{kHz}<f_{\text {Nyquist }}<500 \mathrm{kHz}$. Typically, the digitization rate used is $1 \mathrm{MHz}$. In the experiments described here the fluctuations were not bursty except during the shear-modified ion acoustic wave growth, but the increased resolution proved to be useful when resolving the spikiness that appeared in some spectra. The $500 \mathrm{kHz}$ upper limit on frequency is suitable for most measurements since the highest measured frequency feature appeared below $350 \mathrm{kHz}$.

Measuring the azimuthal wavevector component $k_{\theta}$ when the probe tips are located at the same $r$ radial position with respect to the plasma column symmetry axis and assuming cylindrical symmetry leads to determining the azimuthal propagation wave number $m=r k_{\theta}$. To confirm the finding, another technique is employed. Two Langmuir probes, separated by a larger distance $(23 \mathrm{~cm})$ are used. While one probe is held fixed, the other one can rotate around its symmetry axis and translate in and out of the plasma. The probe alumina tube is mounted at a $20^{\circ}$ angle with respect to the probe axis. Thus, the probe tip can be positioned anywhere on a two-dimensional cylindrical shell within the plasma column, practically sweeping the entire plasma column cross-section while keeping the distance between it and the fixed probe approximately unchanged. Ion saturation current fluctuations are measured 
separately by each probe and the phase difference is determined using the two time series. The phase difference is computed using four measurements at each spatial location. A map of the phase difference as a function of position $(x, y)$ is created. The spatial array consists of a $6 x 6$ $\mathrm{cm}^{2}$ interrogation area in which the probe is displaced every $1 \mathrm{~cm}$.

In Fig. 9, an example of the spatial dependence of the phase difference is presented. The values of $\Delta \Phi$ at each grid square corner are displayed. The structure shown is an indication that there is approximately $180^{\circ}$ phase difference between the diagonally opposed points of coordinates $(2 \mathrm{~cm} ;-2 \mathrm{~cm})$ and $(-1 \mathrm{~cm} ; 2 \mathrm{~cm})$ which indicates an $m=1$ azimuthally propagating mode. The relative phase at the points $(1,1),(1,0),(2,1)$ and $(2,0)$ that average to approximately $170^{\circ}$ and the diagonally opposed points $(-1,-1),(-1,-2),(0,-1)$ and $(0,-2)$ that average to approximately $55^{0}$ is consistent with this $m=1$ picture. A possible cause of the asymmetry of the figure will be discussed in chapter V.

It was found that when inserting the probe shaft into the plasma region where the instability is present, the perturbation sometimes causes a frequency downshift proportional to the inserted length. Thus, the type of measurements described in the previous paragraph might cause the excitation of other wave modes, by changing the boundary conditions and thus affecting $k_{z}$ and/or $k_{\theta}$. Great care must then be used when analyzing the data acquired, in order to rule out the possibility of multiple simultaneous eigenmodes existing at the same time during the measurement. Using the $\boldsymbol{k}$-spectrum to determine the components of the wavevector is a method with which multiple eigenmodes can be distinguished. The primary advantage is displaying which values of $\boldsymbol{k}$ are associated with large amplitude fluctuations just as the frequency spectrum is used to identify the frequency of each eigenmode using its amplitude. Such $\boldsymbol{k}$-spectra acquired when the probe array is oriented along $z$ or along $\theta$ resolve the various eigenmodes according to their axial and azimuthal propagation characteristics [Carroll, 1997].

An important aspect of Langmuir probe operation in heavy-atom earth-alkali metals (i.e., barium) is the continuous probe contamination. Barium ions deposited on a probe's metal surfaces create, through recombination, a coating which changes drastically the probe resistive and capacitive characteristics. It has been found that biasing the probe to $-200 \mathrm{~V}$ (extreme ion saturation) to bombard the probe with plasma ions is the most efficient way to remove the contaminants [Amatucci et al., 1993]. Although a decontamination method that relies on probe heating is found to work better with alkali metals because they have low sublimation 
temperatures that are easy to achieve with this technique, barium has a sublimation temperature too large for the method to be effective [Amatucci et al., 1993].

\section{IV.A.2 Single-sided probes}

Single-sided probes are used to measure the relative parallel drift velocity between the electrons and the ions. They are built in a similar way to the Langmuir probes, but the main difference consists in the current-collecting area. In the single-sided probes, the tungsten wire does not extend outside the alumina tube, but is entirely nested in it. A small region of the tube, close to its end that is immersed in the plasma, is then removed (by polishing the tube outer wall) until only half of the tube cross-section is left in place. Thus, the wire can be exposed to the particle flux directionally, since the remaining portion of the tube prevents particles from reaching the wire. By properly adjusting the collecting area perpendicular to the particle drift direction, their drift velocities can be measured. This technique can be successfully used in magnetized plasmas, since the particles drift along the magnetic field lines, and the collecting area is exposed perpendicular to the lines.

The electron parallel drift velocity is determined in the following manner. The probe is biased at a positive voltage (usually $+10 \mathrm{~V}$ to assure that only electrons are collected) in order to measure $I_{e}$ sat and is radially moved across the plasma column in steps of $1 \mathrm{~mm}$. The $I_{e}$ sat dependence on the radial position $r$ is thus recorded and this current is labeled $I_{u p}$. After a complete radial scan, the probe is rotated by $180^{\circ}$ such that the collecting area faces the electrons drifting in the opposite direction from the former case. The saturation current corresponding to the electrons drifting in the opposite direction is then recorded as a function of radial position. This is $I_{d o w n}$. As shown by Gekelman and Stenzel [1978], the ratio of the two currents is related to the ratio between the electron drift speed $v_{d e}$ and the electron thermal speed $v_{t e}$ in the following manner:

$$
\frac{I_{u p}}{I_{d o w n}}=\frac{\exp \left(-\varsigma^{2}\right)+\sqrt{\pi} \varsigma(1+\operatorname{erf}(\varsigma))}{\exp \left(-\varsigma^{2}\right)-\sqrt{\pi} \varsigma(1-\operatorname{erf}(\varsigma))}
$$

where $\zeta=\left|v_{d e} / v_{t e}\right|$. This dependence is shown in Fig. $10 \mathrm{~b}$. 
Equation (105) has a unique solution for $\zeta$. Thus, finding $\zeta$ means determining the parallel electron drift speed $v_{\text {de }}$ in terms of the electron thermal speed $v_{\text {te }}$. Combining the information contained in Fig. 10a and in Fig. 10b, knowing that $v_{t e} / v_{t i}=\left(m_{\mathrm{i}} T_{\mathrm{e}} / m_{\mathrm{e}} T_{\mathrm{i}}\right)^{1 / 2}$ and using $T_{\mathrm{e}} / T_{\mathrm{i}}=1.2$ (a value commonly found in the experiments presented in this dissertation) and $m_{\mathrm{i}} / m_{\mathrm{e}}=2.5 \times 10^{5}$ (valid for barium), the electron drift speed radial profile is plotted and expressed in terms of the ion thermal speed, as shown in Fig. 10c. The direction of the electron drift is the direction of $I_{u p}$, the largest of the two currents $I_{u p}$ and $I_{d o w n}$, respectively. A typical uncertainty in $\zeta$ when using this method in a $\mathrm{Q}$ machine plasma is $10 \%$.

\section{IV.B Emissive probes}

Emissive probes consist of a double-bore alumina tube, through which copper wires are fed and a tungsten filament inserted at the end of the tube. Because the filament is incandescent during the probe operation, it emits electrons through the Richardson effect, thus creating a region of negative space charge that attracts ions. At high emission currents and with a negative bias on the probe, the floating potential of the probe approaches the plasma potential within $15 \%$ since the current-voltage characteristic curve becomes much more symmetric (i.e., the collected ion current is almost equal to the collected electron current). Using a high-impedance voltmeter to determine the floating potential leads then to an estimate of the plasma potential. So, the method of the emissive probe offers a single-point measurement for estimating the plasma potential.

Using the 512-point current-voltage characteristic of a Langmuir probe would yield the same result, but this latter technique takes 512 times longer. Alkali layers on a cold, nonemissive, probe surface introduce a contact potential $\Phi$ which can be as large as $-3 \mathrm{~V}$. Generally, the floating potential of a probe is determined by the space potential, the electron temperature and the contact potential:

$$
\Phi_{f}=\Phi-\ln \left[\left(m_{i} / m_{e}\right)^{1 / 2} / \alpha_{i}\right] K T_{e} / e+\Phi_{c}
$$

[Motley, 1975], where $\alpha_{i}$ is the ratio of the ion to the electron collection area. For low $\left(<10^{15}\right.$ $\mathrm{cm}^{-2} \mathrm{~s}^{-1}$ ) ion flux striking the probe, the alkali layers and the contact potential can be eliminated by heating the probe to one hundred degrees Celsius, but taking care to eliminate 
secondary emission of electrons. Heating the tungsten loop of the probe eliminates the electron temperature dependence of $\delta n$ and $\delta V$. The loop is designed such that the potential drop along the loop portion in contact with the plasma is minimized (the loop is only $2.5 \mathrm{~mm}$ long).

Nevertheless, due to the electron emission, the influence of the emissive probes on the plasma is less localized and they could disturb other probes nearby. This is why the other probes are located at least $23 \mathrm{~cm}$ from the emissive probe (in these experiments) and the emissive probe is only used to determine the equilibrium plasma potential.

\section{IV.C Laser induced fluorescence diagnostics}

Laser induced fluorescence diagnostics represent a major development in plasma diagnostics. They are a direct, non-perturbative and non-invasive technique which provides spatially localized measurements of the ion velocity distribution, ion temperature and ion density (in metastable states). Laser induced fluorescence uses a laser tuned to a natural absorption line of an ion to induce emission from one state to another via an intermediate step wherein the electrons are pumped temporarily to a higher state. The emission from this higher state is monitored as the laser frequency is swept to obtain the LIF lineshape. From the LIF lineshape, the ion velocity distribution can be determined.

The first LIF measurements in a plasma were used to obtain the local ion velocity distribution function and relative density of the metastable ions [Stern and Johnson, 1975]. They utilized a fixed-frequency argon-ion laser and used the correlation of the angles between the laser beam, viewing axis and field gradients in order to obtain information on different sections of the velocity distribution. Presented were measurements taken both parallel and perpendicular to the magnetic field applied in the device used, which was a low-pressure hotcathode dc discharge in argon. Previously, Rynn et al. [1974] had used a single-mode argon laser to calibrate a pressure-swept Fabry-Perot interferometer in order to measure the BaII ion temperature in the presence of a current-driven electrostatic ion-cyclotron (CDEIC) wave. They operated a single ended Q machine and observed the increase in the ion temperature perpendicular to the applied magnetic field in the presence of CDEIC waves by noticing the broadening of the emitted $\sigma$ Doppler lines, thus directly documenting for the first time the 
CDEIC wave-induced ion heating. The first spectroscopic measurements of BaII plasmas produced in a Q machine was reported in 1963 by Hinnov et al. [1963], who used the same apparatus to document the perpendicular ion-heating in the presence of plasma currents.

With the introduction of tunable dye lasers in the plasma diagnostics by Hill et al. [1983], the task of taking ion velocity distribution, ion density, ion temperature and ion particle-tracking (optical tagging) measurements has become a straightforward operation. The laser frequency is swept over a narrow frequency range containing an ion absorption transition, which leads to the excitation of the plasma ions. The excited state decays spontaneously, therefore the ions de-excite by making a downward transition through which they emit the absorbed laser light or a different wavelength light. The absorption spectrum of the ion metastable states is determined by measuring the intensity of the fluorescent emission as a function of laser frequency. The absorption spectrum can experience Doppler broadening due to the ion motion and can experience Zeeman split due to the presence of the applied plasma-confining magnetic field. The emission region is localized within the narrow spatial region of the laser beam in the plasma and the collection optics used is calibrated such that it can collect the light emitted from a very localized region in the plasma that is of the order of 5 $\mathrm{mm}^{3}$. Using this kind of a device, the density of the metastable state, the ion temperature and the ion net drift velocity can be determined from the frequency dependence of the measured emission spectrum. The metastable state density can be determined by using a detector which was previously absolutely calibrated.

There is another more recent [Skiff and Anderegg, 1987; Safarty et al., 1996] purpose for using laser induced fluorescence measurements in plasmas. As shown by Skiff and Anderegg, the perturbations to the ion distribution function due to the interaction between ions and electrostatic waves can be detected using LIF. By fitting theoretical curves to the perturbed ion distribution function measured, the wavevector components can be determined.

Parallel-ion-velocity shear was first measured using LIF by Skiff and Fasoli [1993]. The relative state density of spin sublevels, changed at one location in the plasma phase space by optical pumping, was subsequently observed by LIF at a second location. Use of a single laser to provide both beams was possible if one beam was used to observe the decrease in the state density produced by the other beam. In the absence of parallel-velocity shear, the measured transfer function had a peak localized on the plasma column axis. In the presence of 
shear, it showed a structured, periodic, multi-peaked signal due to the cross-field particle transport enhanced by parallel-velocity shear. No other way of verifying the presence of shear was presented in the work. An actual measurement of the spatial dependence of the ion parallel drift velocity was not reported.

\section{IV.C.1 Laser induced fluorescence apparatus}

In this section, the laser induced fluorescence apparatus used in the experiments performed on the WVU Q machine is presented. For the interested reader, a more complete description of LIF theory, apparatus and techniques in $\mathrm{Q}$ machines can be found in the dissertation of Hill [1983].

The laser-induced fluorescence apparatus had been set up prior to the experiments presented in this dissertation. It was designed such that either parallel or perpendicular measurements could be performed. The first perpendicular LIF measurements were reported by Koepke et al., [1998] who presented the radial profile of the $\boldsymbol{E} \times \boldsymbol{B}$ ion drift velocity and the radial profile of the perpendicular ion temperature when ion cyclotron waves excited by the inhomogeneous energy-density driven mechanism were observed. Parallel LIF measurements were reported by Koepke et al. [2000] when studying the radial profile of the parallel-ion-drift velocity in the Double Ionizer Double Electrode (DIDE) configuration of the Q machine.

The LIF laser system, presented in Fig. 11, is comprised of a 6 W Coherent Innova 90 Plus, continuously-operated, argon-ion laser which optically pumps a Coherent 899 ring laser in which population inversion in achieved. The latter is a tunable dye-laser using Rhodamine $6 \mathrm{G}$, an organic dye solution, as the lasing medium. Among the various plasma ion species that can be produced in a Q machine, BaII has a transition at $585.4 \mathrm{~nm}$, between the atomic levels $5{ }^{2} \mathrm{D}_{3 / 2} \rightarrow 6{ }^{2} \mathrm{P}_{3 / 2}$, well within the operational range of Rhodamine $6 \mathrm{G}$. Therefore the ions excitation is produced by the absorption of the $585.4 \mathrm{~nm}$ wavelength radiation from the tunable dye laser; the observed spectrum is due to ion de-excitation in the $6{ }^{2} \mathrm{P}_{3 / 2} \rightarrow 6{ }^{2} \mathrm{~S}_{1 / 2}$ transition which takes place at $455.52 \mathrm{~nm}$ wavelength. In $\mathrm{Q}$ machines producing barium plasma, some $80 \%$ of the barium ions produced by contact ionization at the hot plate are in the ground state $\left(6{ }^{2} \mathrm{P}_{1 / 2}\right)$, almost $8 \%$ are in the $5{ }^{2} \mathrm{D}_{5 / 2}$ metastable state, around $12 \%$ are in the 5 
${ }^{2} \mathrm{D}_{3 / 2}$ metastable state, and a negligible fraction are in higher states as estimated from the Saha equation [Hill, 1983]. The stability of the laser operation at this wavelength and the dye-laser output power are also reasons for using LIF between the atomic levels mentioned above and for using Rhodamine $6 \mathrm{G}$ as a lasing medium. The input pumping power, usually $4.5 \mathrm{~W}$, produces alteration of the Rhodamine such that it must be replaced approximately every three months.

The wavelength of the tunable dye laser is swept by $\Delta \lambda=0.02 \mathrm{~nm}$ (or $\Delta f=15 \mathrm{GHz}$ ) increments around the value of $585.4 \mathrm{~nm}$. This is accomplished using the computer-controlled Coherent dye-laser control unit by applying a $\pm 5 \mathrm{~V}$ signal. The computer generated data array used for storing the information was set to have either 100 data points or 512 data points. The former is designed to be used in the case of light emission with a high level of signal to noise, when the peak of the signal is easily detectable, whereas the latter is for the cases when the ratio is small, and detecting the peaks in the signal is more challenging.

The tunable laser light wavelength is monitored using a Burleigh 1500 wavemeter by splitting $10 \%$ of the laser output light and directing it into the wavemeter. Some $5 \%$ of this split light is directed into a Photonics 104 barium hollow cathode lamp. The purpose is to provide a BaII zero-velocity drift reference for the measurements. The lamp is designed to provide a light signal from the stationary BaII population inside in the same way the plasma ions respond to the laser induced fluorescence mechanism. The Ba plasma inside the lamp is obtained by a hot-cathode glow discharge method. The voltage applied using a Kepco dc power supply is $200 \mathrm{~V}$ and the current collected is almost $14 \mathrm{~mA}$. The fluorescence signal obtained from the optically pumped states of the BaII ions in the lamp is measured indirectly, through the optogalvanic variations of the discharge current, with an EG\&G Princeton Applied Research lock-in amplifier and recorded for each measurement taken.

A mechanical chopper, set at a frequency of $f=2.5 \mathrm{kHz}$, is used to chop the tunable laser output light. The purpose of the chopper is to extract the fluorescence signal from the background light that contains the same wavelength. The light used to excite the hollowcathode lamp ions is thus $2.5 \mathrm{kHz}$-chopped and the lock-in amplifier is synchronized by an input signal at the same frequency. Calibration measurements made using an iodine cell in order to determine ion motion in the hollow-cathode lamp show that their drift speed could be as large as $v_{d i} \leq(41 \pm 4) \mathrm{m} / \mathrm{s}$, a quantity which is much smaller than both the ion thermal speed 
and the ion drift speed in the Q machine [T. Good, private communication]. Laser induced fluorescence measurements done using both the hollow cathode lamp and the iodine cell, during which spectra of both devices were recorded simultaneously for each measurement and the spectral position of the equivalent lines was measured, show that in the vast majority of cases, the $\mathrm{Ba}$ ions in the lamp were drifting at speeds $v_{d i} \sim 15 \mathrm{~m} / \mathrm{s}$. [T. Good, private communication].

The tunable dye-laser output is put into a fiber optic cable $(200-\mu \mathrm{m}$ diameter) which transports the light to the injection optics mounted on the WVU Q machine. There are two injection optics used in these experiments. One is for injecting the laser beam quasi-parallel to the symmetry axis of the WVU Q machine (i.e., along the external magnetic field lines), referred to as the "parallel injection optics" and is presented in Fig. 12. The other one, the "perpendicular injection optics", is used to inject the laser beam perpendicular to the magnetic field lines and is shown in Fig. 13. All injection and collection optics are mounted on Qmachine view ports. No further design modifications are needed since the injection fiber optic cable is easily connected to either one of the two injection optics. The collection optics are mounted on the $\mathrm{Q}$ machine at the same axial position as the perpendicular injection optics (Fig. 13) but at an angle of $90^{\circ}$ around the chamber circumference.

The perpendicular injection optics and the collection optics are mounted on translation stages operated by computer-controlled Velmex motor controllers and Vexta motors at $46 \mathrm{~cm}$ from the WVU Q machine north end. Of the two motors, one translates both the injection optics and simultaneously the collection optics in the $x$ direction for perpendicular measurements $\left(v_{i y}\right)$ at various $x$ positions. The other motor translates the injection optics along $y$-axis. When both the injection and the collection optics are moved along the $x$ direction, keeping the collection optics at the $y=0$ position, the azimuthal component of the ion drift velocity $v_{\theta}$ is measured. As mentioned above, the other motor translates the collection optics in the $y$ direction for detecting the photons during the perpendicular measurements at a given $x$. When keeping the injection optics at $x=0$ and scanning the collection optics along $y$, the radial component $v_{r}$ is determined. This allows for complete mobility in the perpendicular measurements.

The parallel injection optics are mounted on a rotational stage, operated and controlled in the same way as the perpendicular injection optics and situated $68 \mathrm{~cm}$ away from the WVU 
Q-machine south end. This arrangement of the two injection optics allows for LIF scans both in the perpendicular direction by moving the collection optics in the $x$ direction and injecting the laser beam along $y$ and in the parallel direction by moving both the $z$-injected (parallel) laser beam and the collection optics along $x$. Thus, the perpendicular LIF measurements provide information regarding the ion metastable state density (which depends on the ion density and electron temperature) across $y$, ion drift $v_{d y}$, and ion temperature $T_{i y}$, while the parallel measurements reveal the ion metastable state density, ion drift $v_{d z}$ and ion temperature $T_{i z}$ across $x$.

Injection optics contain lenses that focus the injection beam such that its width is not larger than $2 \mathrm{~mm}$ when injected in the perpendicular direction, across the plasma column, which is typically $6 \mathrm{~cm}$ wide. In the parallel direction there is a larger distance between the injection and the focus locations, a distance that is $1.7 \mathrm{~m}$. To aim the laser beam quasi-parallel to the plasma's cylindrical axis, a fixed periscope mirror $M$ is used in the parallel injection optics to reflect the radially entering light from the telescope to the quasi field-aligned path to the focus location. The goniometer in the injection optics adjusts the incident light at a variety of angles such that it always strikes the mirror at the same point while controlling the quasiparalllel angle to arrive at a linear relationship between the radius (from the cylindrical axis) of the focus position and the number of steps required for moving the stepper motor by a suitable amount. The mirror is a $1 " \mathrm{x} 1$ " x 0.050 " polished-surface mirror and the goniometer is a dual-stage theta-phi table from OptoSigma. The Vexta motor driving the stage is attached to a worm-gear assembly that rotates the $x$-axis of the goniometer. The second stage of the table is used for centering the laser beam in the $y$ direction. This in turn positions the beam mostly parallel to the magnetic field at different radial $(x)$ locations that are tracked with the collection optics by moving the optics along the $x$ direction.

The resonant laser light injected at $585.53 \mathrm{~nm}$ vacuum wavelength (approx. $585.37 \mathrm{~nm}$ wavelength in air) excites the ions in the $5{ }^{2} \mathrm{D}_{3 / 2}$ metastable state (see Fig. 14b). This is a metastable state since the ion cannot perform decaying transitions to the $5{ }^{2} \mathrm{~S}_{1 / 2}$ ground state except through electric quadrupole radiation. Since the probability of quadrupole radiations is small, the $5{ }^{2} \mathrm{D}_{3 / 2}$ state is a metastable one with a lifetime of the order of $10 \mathrm{~s}$ [Hill, 1983]. After reaching the excited state $6{ }^{2} \mathrm{P}_{3 / 2}$, the ions decay spontaneously (decay rate $1.18 \times 10^{8}$ $\mathrm{s}^{-1}$ ) to the ground state $6{ }^{2} \mathrm{~S}_{1 / 2}$ state emitting a fluorescence radiation at $455.52 \mathrm{~nm}$ vacuum 
wavelength (Fig. 14a). Since the ions are in the externally applied magnetic field present in every $\mathrm{Q}$ machine, the decay transition fine structure consists of several lines. The upper $6{ }^{2} \mathrm{P}_{3 / 2}$ level is split into four sublevels; the ground level is split into two sublevels, as shown in Fig.14a and Fig. 14b. This fine structure is the result of the interaction between the ion spinmagnetic and orbital-magnetic moments, respectively. With the low (much below $20 \mathrm{~T}$ ) external magnetic field, the interaction energy is proportional to $\mathbf{J \bullet B}$. Here, $\mathbf{B}$ is the external magnetic field, and $\mathbf{J}=\mathbf{L}+\mathbf{S}$ is the ion total angular moment vector. Thus, the fluorescence decay $6{ }^{2} \mathrm{P}_{3 / 2} \rightarrow 6{ }^{2} \mathrm{~S}_{1 / 2}$ is possible through a total of ten transitions. Of them, six are $\sigma$ transitions (with $\Delta m_{j}= \pm 1$ ) and four are $\pi$ transitions $\left(\Delta m_{j}=0\right)$. The metastable state is also split into four sublevels. Therefore the pumping to the upper level could be made through ten transitions.

In the case of parallel injection, since the laser beam is parallel to the magnetic field, its electric-field vector is always perpendicular to the magnetic field, and so the excitation is made by means of pumping all the six $\sigma$ transitions. The perpendicular injection optics contain a polarizer so fewer transitions will be excited, depending on the orientation of the polarizer with respect to the magnetic field line. As used by E. Reynolds [Ph.D. dissertation, in preparation], the polarizer was oriented parallel to the magnetic field lines. When the perpendicular injection optics is used, i.e., the laser beam is injected perpendicularly, the polarizer only allows for the laser beam electric-field vectors which are parallel to the magnetic field lines to pass, meaning that the $5{ }^{2} \mathrm{D}_{3 / 2} \rightarrow 6{ }^{2} \mathrm{P}_{3 / 2}$ excitation is made by $\pi$ transitions. The $50 \%$ loss of signal caused by including the polarizer is acceptable. Irrespective of the injection optics used (parallel or perpendicular), only the four $\pi$ stimulated decay transitions are observed. This is so because the collection optics receive photons travelling perpendicular to the magnetic field, and the polarizer used in the collection optics is oriented parallel to the magnetic field lines. Due to the specific fine structure of the atomic levels involved in the absorption and emission transitions shown in Fig. 14, it is possible to eliminate lines from the emission spectrum without losing information about the absorption spectrum.

In the case of parallel injection, as mentioned before, only six $\sigma$ absorption transitions, shown in Fig. 15, are excited. They decay as $\sigma$ and $\pi$ emission (fluorescence) transitions whose spectrum is given by the absorption spectrum but whose polarization is determined in 
the emission diagram. The observed spectrum will consist of a superimposed combination of $\sigma$ and $\pi$ transitions with wavelengths set by the Lande factor $g_{L}$ of the absorption diagram shown in Fig 14b. When using the collection optics polarizer oriented parallel to the magnetic field, all photons, irrespective of their frequency, emitted via $\sigma$ transitions will be blocked. Thus, the spectrum intensity will be lowered, but since the outer absorption $\sigma$ lines can only decay via $\sigma$ transitions (see Fig. 15) they will be completely eliminated from the emission spectrum, making the analysis easier.

When injecting the laser beam perpendicular to the magnetic field and aligning the injection optics polarizer parallel to the magnetic field, only four $\pi$ transitions will pumped, as shown in Fig. 16. They can decay solely as $\sigma$ transitions (the two outer ones) and as $\sigma$ and $\pi$ transitions (the two inner ones). The presence of the polarizer parallel to the magnetic field in the collection optics eliminates the $\sigma$ transitions, so only two $\pi$ lines will be observed.

Simplifying the observed fluorescence spectrum by using a polarizer is important in the case of the transitions used in these LIF measurements, since, as will be shown later, the absorption lines are broadened by Doppler broadening and power broadening, aside from the line natural width and they are subject to Zeeman splitting. The combined result of these effects burdens the work of finding the ion velocity distribution, which is significantly eased in the case of a simpler observed spectrum. The same task of simplifying the observed spectrum might have been accomplished using a quarter-wavelength plate in the collection optics, which if properly positioned, would have eliminated half of the $\sigma$ transitions (either right- or left-hand polarized meaning $\Delta m_{J}=1$ or -1 , respectively). This method was not employed because the observed spectrum would have been asymmetric, making the finding of the zero-velocity reference more difficult.

To minimize the background light and reflected light collected at the collection optics, three baffles are used. They are positioned across the plasma column fron both the perpendicular injection optics and the collection optics, at which locations the blank flange is blackened, as well as around the collection-optics viewport. The collected fluorescence light is transported through a fiber optic cable to a photomultiplier tube (PMT). A $10 \AA \AA$ band-pass filter centered at $455.4 \mathrm{~nm}$ is used in front of the PMT. This assures that only the $6{ }^{2} \mathrm{P}_{3 / 2} \rightarrow 6$ ${ }^{2} \mathrm{~S}_{1 / 2}$ transition is observed. The electric signal of the PMT is transported to a Stanford Research SR830 lock-in amplifier. The amplifier also receives the hollow-cathode lamp 
signal. The amplifier output signal is normalized to the laser power signal to account for signal power variations during the frequency sweeps. For each tunable laser frequency, the output power signal from the dye laser, the signal from the hollow-cathode lamp, the output of the lock-in amplifier and the sweep voltage used to control the tunable dye laser are monitored and recorded with a $100 \mathrm{ksample} / \mathrm{s}$ digitizer. Control over the digitizer is achieved with an ASYST code that stores the data in 100 or 512 data point arrays.

The image size at the focus position is sought to be no larger than $2 \mathrm{~mm} \times 2 \mathrm{~mm}$. Therefore, the parallel injection optics telescope consists of two lenses, a diverging one $\left(f_{l}=-\right.$ $25 \mathrm{~mm})$ and a converging one $\left(f_{2}=60 \mathrm{~mm}\right)$, an assembly that can combine the two desired effects: an image of a given size and an image distance of a certain value. These focal lengths were found by optimizing the telescope assembly for the minimum image size and reasonable lens separation, which is also linked to the image size. The laser beam is injected into the $\mathrm{Q}$ machine at an adjustable angle $\alpha \approx 4^{\circ}$, consistent with a quasi-parallel injection. The effect of the beam angle on measured versus real ion parallel temperature, for instance, is checked by considering a bi-Maxwellian distribution of known parallel and perpendicular temperature and then projecting the distribution at the angle of the incident beam [M. Zintl, private communication]. The graph in Fig. 17 shows the comparison between the measured temperature $T_{\theta}$ and the "true" temperature $T_{\perp}$, and it can be seen that the measured temperature should increase both with perpendicular temperature and with increased beam angle relative to the magnetic field. Setting this angle below $7^{\circ}$ assures the reproducibility of temperature measurements for cases in which $1 \leq T_{\perp} / T_{\|} \leq 10$. To do so, the distance between the parallel injection optics and the collection optics must be at least $1.50 \mathrm{~m}$; in these experiments, it is $1.70 \mathrm{~m}$, while $1<T_{\perp} / T_{\|}<2.5$. With the existing set-up of the injection and collection optics, LIF radial scans can be performed in an array of $6.4 \mathrm{~cm} \times 6.4 \mathrm{~cm}$ over the entire cross section of the plasma column.

\section{IV.C.2. Laser induced fluorescence data analysis}

Data analysis consists in determining the ion distribution function from the observed fluorescence spectrum and, from it, extracting information regarding the ion drift velocity, ion 
temperature and metastable state ion density. To determine the distribution function, a fitting routine is used. The fitting starts from the assumption that the population has a Maxwellian dependence on velocity, i.e.,

$$
f(v)=\left(m / 2 \pi k_{B} T_{i}\right)^{1 / 2} \exp \left(-\frac{m\left(v-v_{d}\right)^{2}}{2 k T_{i}}\right)
$$

where $m$ is the ion mass, $T_{\mathrm{i}}$ is the ion temperature and $v_{\mathrm{d}}$ is the ion drift velocity. Since LIF measurements are done along one dimension at a time, the one-dimensional Maxwellian distribution is employed in the fitting routine. Non-Maxwellian modifications to the initial Maxwellian distribution fitting are made when the fitting is not satisfactory.

Several aspects must be carefully addressed when performing the fitting routine. The observed Doppler shifted lines can be broadened and split by several effects. The most obvious one, when dealing with plasmas created in a $\mathrm{Q}$ machine at 0.1 to $0.3 \mathrm{~T}$ magnetic fields, is the Zeeman splitting. From the expression of the ion-magnetic field interaction energy

$$
\Delta H=\mu_{\mathrm{B}} B g_{\mathrm{Lj}} m_{\mathrm{j}},
$$

where $\mu_{B}=e h /\left(4 \pi m_{e}\right)$ is the Bohr magneton ( $h$ is the Planck constant), $m_{j}$ is the orbital magnetic quantum number and $\mathrm{g}_{\mathrm{Lj}}$ is the Lande factor, the energy difference between levels 1 and 2 is

$$
\Delta H_{12}=\mu_{\mathrm{B}} B\left(g_{\mathrm{L} 2} m_{2}-g_{\mathrm{L} 1} m_{1}\right)
$$

or

$$
\Delta \lambda=\mu_{\mathrm{B}} B \lambda^{2}\left(g_{\mathrm{L} 2} m_{2}-g_{\mathrm{L} 1} m_{1}\right) / h c .
$$

Given $\mathrm{B}=0.1 \mathrm{~T}$, the splitting of the $\pi$ lines is

$$
\Delta \lambda=3 \times 10^{-3} \mathrm{~nm}
$$

The Stark splitting is negligible, since the electric fields in these experiments are smaller than $10 \mathrm{~V} / \mathrm{m}$ in the plasma regions where the fluorescence signal is collected. The fact that the plasma density is not high $\left(n_{0} \leq 10^{9} \mathrm{~cm}^{-3}\right)$ reduces any other contribution from the Stark splitting to the observed spectrum. The Stark splitting can be expressed in terms of wavelength as

$$
\Delta \lambda=\frac{3 h \lambda^{2}}{8 \pi^{2} c} \frac{n_{p} E}{m_{i} Z e}\left(n_{1}-n_{2}\right) .
$$


In this first order approximation formula, $n_{\mathrm{p}}$ is the principal quantum number, $n_{1}$ and $n_{2}$ are the parabolic quantum numbers $\left(n_{1}+n_{2}<n_{\mathrm{p}}\right)$ and $E$ is the applied external electric field. For the maximum observed electric field, $E=70 \mathrm{~V} / \mathrm{m}$,

$$
\Delta \lambda_{\max } \sim 2 \times 10^{-4} \mathrm{~nm} \text {. }
$$

A typical value is

$$
\Delta \lambda \sim 3 \times 10^{-5} \mathrm{~nm} .
$$

Power broadening, occurring when the stimulated emission rate equals the absorption rate and when both are larger than the spontaneous emission rate, is estimated to be negligible. This is so since the spontaneous emission rate of the observed transition is larger than both the stimulated emission and the absorption rates.

The natural line width of a transition between an excited state and the ground state is, according to Heisenberg's uncertainty principle,

$$
\Delta H \geq h /(2 \pi \tau)
$$

where $\tau$ is the mean lifetime of the excited state. If the transition occurs between two excited states $i$ and $k$, the total minimum uncertainty is

$$
\Delta H=\Delta H_{i}+\Delta H_{k}
$$

In terms of frequencies, this is

$$
\Delta \omega_{i k}=\Sigma_{m} A_{i m}+\Sigma_{n} A_{n k}
$$

which includes all transitions to any of the two levels of interest, with $A_{\text {im }}$ and $A_{n k}$ being the Einstein transition coefficients. In the case of $\mathrm{Ba}$ ions, the tabulated values [Hill, 1983] of coefficients yield

$$
\Delta \omega_{i k}=1.22 \times 10^{8} \mathrm{~s}^{-1}
$$

or as wavelength

$$
\Delta \lambda \approx 8.51 \times 10^{-5} \mathrm{~nm} .
$$

The pressure broadening effects of ion-neutrals collisions is only significant for weakly ionized plasmas (ionization percentage $\leq 1 \%$ ). Thus it is insignificant for highly ionized plasmas such as Q machine plasmas. The instrumental broadening, associated with the laser linewidth and, separately, with the dispersion of the light collection system, is also negligible. Due to the use of the $1 \mathrm{~nm}$ bandpass filter in the collection optics there is no 
dispersion in the collection system and the $1 \mathrm{MHz}$ laser bandwidth yields a broadening in terms of wavelength equal to

$$
\Delta \lambda \leq 1.2 \times 10^{-6} \mathrm{~nm} .
$$

The thermal ions produce Doppler shifting of the transition frequency from the proper value $\omega_{0}$ to

$$
\omega=\omega_{0} \frac{c+v_{\text {obs }} \cos \theta_{\text {obs }}}{c-v \cos \theta_{\text {source }}}
$$

where $\omega$ is the Doppler shifted frequency, $c$ is the speed of light, $v_{o b s}$ is the speed of the observer, $\theta_{o b s}$ is the angle between the observer's trajectory and the line connecting the instantaneous positions of the source and the observer, $v$ is the speed of the ions and $\theta_{\text {source }}$ is the angle between the source's trajectory and the line connecting the instantaneous positions of the source and the observer. This non-relativistic formula $\left(c>>v, c>>v_{o b s}\right)$ becomes

$$
\omega=\omega_{0} \frac{c}{c \mp v}
$$

for a stationary observer $\left(v_{o b s}=0\right)$ and ions travelling towards/away from the observer. Therefore, the frequency Doppler shift is proportional to the ion motion and is given by

$$
\begin{gathered}
\Delta \omega=\omega-\omega_{0}=\omega_{0} \frac{1}{1 \mp \frac{v}{c}}-\omega_{0} \approx \omega_{0}\left(1 \pm \frac{v}{c}\right)-\omega_{0}= \pm \omega_{0} \frac{v}{c} \\
|\Delta \omega| \approx \omega_{0} \frac{v}{c} .
\end{gathered}
$$

The absorbed/emitted lines from a Maxwellian ion population drifting with the velocity $v_{0}$ are thus Doppler broadened. Since for ions at the drift speed $v_{0}$ the frequency Doppler shift is $\left|\Delta \omega_{0}\right| \sim \omega_{0} v_{0} / c$ and for ions at a particular speed $v$ the shift is $\left|\Delta \omega^{2}\right|=\omega_{0} v / c$, it follows that the absorption/emission transition dependence on frequency will be:

$$
I(\omega)=I_{\max } \exp \left(-\frac{m_{i} c^{2}\left(\omega-\omega_{0}-\omega_{0} \frac{v_{0}}{c}\right)^{2}}{2 \omega_{0}^{2} k T_{i}}\right) .
$$


The intensity $I(\omega)$ of the Doppler-broadened absorption line is determined in LIF measurements as a function of frequency $\omega$. To determine the distribution function from the absorption lineshape, the single frequency $\omega$ of the laser is swept in small increments. The increment size (in wavelengths) is $1.7 \times 10^{-4} \mathrm{~nm}$. Only the ions that satisfy the resonance condition:

$$
\mathbf{k} \cdot \mathbf{v}=\omega-\omega_{0}
$$

will interact with the laser light. Here, $|\mathbf{k}|=\omega / 2 \pi c$ is the input laser light wave number. Thus, the absorption rate is velocity dependent,

$$
W(\mathbf{v})=\frac{\lambda_{0} I_{\max }}{8 \pi h \omega_{0}} \tau^{-1} \Lambda(\omega-\mathbf{k} \cdot \mathbf{v})
$$

where $\tau$ is the lifetime of the radiative upper state and $\Lambda$ is the ion resonance function,

$$
\Lambda(\omega-\mathbf{k} \cdot \mathbf{v})=\frac{1 /(2 \pi \tau)}{\left(\omega-\mathbf{k} \cdot \mathbf{v}-\omega_{0}\right)^{2}+1 / 4 \tau^{2}}
$$

The total number of photons emitted by the plasma per second per unit volume per solid angle, irrespective of their emission frequency, is

$$
\frac{d N}{d \Omega}=\iint d \mathbf{v} d \omega W(\mathbf{v}) f(\mathbf{v})=\frac{1}{8 \pi \tau^{2}} \frac{I_{\max }}{h \omega_{0}} \lambda_{0} F\left(\frac{\omega-\omega_{0}}{k}\right),
$$

where

$$
\begin{aligned}
& F\left(v_{k}\right)=\int f_{0}\left(v_{k}, \mathbf{v}_{\perp}\right) d \mathbf{v}_{\perp}, \\
& v_{k}=\hat{\mathbf{k}} \cdot \mathbf{v} \equiv \frac{\omega-\omega_{0}}{k} \text { and } \mathbf{v}_{\perp}=\mathbf{v}-(\mathbf{v} \cdot \hat{\mathbf{k}}) \hat{\mathbf{k}}
\end{aligned}
$$

Here, $f_{0}$ is the ion velocity distribution. Eq. (129) is used to absolutely calibrate the detector to measure the ion metastable-state density, which was not done in the experiments presented in this dissertation.

To find the one-dimensional distribution function along $\mathbf{k}$, it is only necessary to scan $\omega$ across the Doppler broadened absorption line, recording the intensity of the laser-induced fluorescence as a function of $\omega$. 
The Zeeman-split removal algorithm was also demonstrated by E. Reynolds [Ph.D. dissertation, in preparation]. It is performed based on knowing the spectral position of the lines in the observed spectrum. At usual barium ion temperatures in $\mathrm{Q}$ machines $(\sim 0.2 \mathrm{eV})$ the Doppler broadening due to the ion thermal motion is on the order of the Zeeman splitting. To precisely determine the spectral line position, the ion population is first cooled with the addition of neutral helium. The elevated helium pressure ( $p \leq 1 \times 10^{-4}$ Torr) leads to an increased ion-neutrals collision rate, so the barium ions lose energy and momentum to the helium atoms. Since the line width is directly proportional to the square root of the ion temperature, a decreased temperature will appear as a narrowed line.

As seen in Fig. 18, the four $\pi$ lines observed in the case of laser parallel-injection are distinguishable. The applied magnetic field is $B=0.3 \mathrm{~T}$. The full width at half maximum of the broadened line is directly proportional to the square root of the ion temperature

$$
\Delta \lambda_{\text {FWHM }}=\left(2 \ln 2 \frac{2 k T_{i}}{m_{i}}\right)^{1 / 2} \frac{\lambda_{0}}{c} .
$$

The vertical error bar represents the uncertainty due to the use of a particular integration time (i.e., photon counting) during the recording of the signal, and the horizontal bar represents the sum of all the broadening mechanisms, except for Doppler broadening. A set of lineshapes is recorded as a function of magnetic field for future reference. Thus, the spectral position of the transition lines is experimentally determined and recorded as a function of magnetic field. Also, this method allows for a confirmation of the relative intensity of the two inner lines versus the outer two ones. The spectral position of the hollow-cathode lamp line is used for zero-velocity reference, corresponding to $\lambda_{0}=585.5301 \mathrm{~nm}$ (in vacuum).

The same method is used in the case of perpendicular laser injection for determining the spectral position of the two $\pi$ lines, observed in this case as a function of magnetic field. Their intensities are equal to each other, which is also verified experimentally.

For both parallel and perpendicular injections, a MATLAB code routine (created by E. Reynolds [Ph.D. dissertation, in preparation]) is used. Given as input parameters are the number of photons counted as a function of frequency or wavelength, the applied magnetic field, the frequency scan width, the ion mass, the Boltzmann constant, and the hollow-cathode lamp line spectral position. The routine performs a least-squares fit to a three-parameterfunction. The fitted function is a Maxwellian and the fitting parameters are the signal 
amplitude $I_{0}$, ion drift velocity, and ion temperature. This fitting is done to each of the observed lines, based on their lineshapes. For the spectrum shown in Fig. 18, the drift speed is $82 \mathrm{~m} / \mathrm{s}$ and the ion temperature is $0.034 \mathrm{eV}$. This drift velocity is very small comparing to the drift velocity of ions at the usual temperature $\left(T_{\mathrm{i}}=0.21 \mathrm{eV}\right)$, which is approximately $v_{\mathrm{di}}=500$ $\mathrm{m} / \mathrm{s}$. As shown by Koepke et al. [2002], the parallel ion drift speed becomes smaller than 50 $\mathrm{m} / \mathrm{s}$ when the neutral pressure exceeds $4 \times 10^{-4}$ Torr.

When operating at the usual Q machine ion temperatures, the Doppler broadening is much larger than in the previous collisional-ion case. Thus, the two inner lines are no longer distinguishable (Fig. 19). Nevertheless, their spectral position and relative intensity is experimentally known for the specific value of magnetic field strength $B$ at which the measurement was performed. Thus, the fitting routine is performed in the manner explained above. The sum of the four fitted lines is plotted (solid line) against the observed LIF signal (dots) for comparison. As seen, the convoluted fitted-lineshape overlaps well the raw data. Below, the fitted ion distribution is plotted. The relative spectral position of the four or two $\pi$ lines with respect to $\lambda_{0}=585.5301 \mathrm{~nm}$ determines the ion drift velocity. The fiting code accounts for the ions drift relative to the magnetic field direction. Here $v_{d i}=520 \mathrm{~m} / \mathrm{s}$ and $T_{i}$ $=0.23 \mathrm{eV}$.

In Fig. 20 is presented the case of the Zeeman-split removal for perpendicular laser injection with $B=0.1 \mathrm{~T}$. The Doppler broadening is much larger than the Zeeman split so the two $\pi$ lines are not distinguishable. The fitting performed by the Zeeman-split removal routine is based on the fact that there are indeed two observed lines, with equal intensities. Here

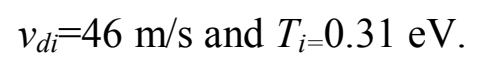

In the double-ended Q machine configuration, ions are produced at both ends of the machine. A parallel LIF measurement will reveal two oppositely drifting ion populations. The spectrum observed in this case shows a slightly more complicated lineshape configuration: the peak labeled 1 in Fig. 21 pertains to the ion population drifting anti-parallel to the magnetic field, the peak labeled 2 to the population drifting parallel to the magnetic field. The middle peak is a superposition of peaks pertaining to both ion populations. The MATLAB code routine used for this type of measurement takes into account the presence of the extra set of peaks. Since the thermalization processes are identical for both populations, the populations are considered to have the same temperature. Thus five parameters (1) signal intensity and (2) drift velocity of population 1, (3) signal intensity and (4) drift velocity of population 2 , and (5) 
ion temperature are used for the fit to the lineshape. The two fitted ion distributions are shown below. The light-grey symbols represent the distribution of the ions propagating anti-parallel to the magnetic field at $v_{d i l}=-885 \mathrm{~m} / \mathrm{s}$, the dark-grey symbols represent the distribution of the ions propagating parallel to the magnetic field at $v_{d i 2}=464 \mathrm{~m} / \mathrm{s}$, and their temperature is $T_{i}=0.21 \mathrm{eV}$. Note that the ratio of the two ion densities, as inferred from their signal amplitudes, is 2.97:1, parallel-propagating ions versus anti-parallel-propagating ions. All ion populations studied in these experiments can be described using drifting Maxwellian velocity distributions. 


\section{Chapter V. Experimental results}

This chapter presents the experimental results of the study on the ion cyclotron and ion acoustic wave characteristics in the presence of parallel-ion-velocity shear. For the case of ion acoustic waves, the effect of ion-temperature anisotropy in their growth and propagation is also presented.

\section{V.A Shear-modified ion cyclotron waves}

\section{V.A.1 Control of the ion parallel drift velocity radial profile}

Control over the ion parallel drift velocity radial profile is achieved in the $\mathrm{Q}$ machine plasma in the Single Ionizer Single Electrode (SISE) configuration. In this configuration, D'Angelo and Motley [1962] first studied current-driven electrostatic ion cyclotron (CDEIC) waves. In the experiment performed in the WVU Q machine, the biasable electrode is inserted in the center of plasma column, at $275 \mathrm{~cm}$ away from the ionizer, meaning $25 \mathrm{~cm}$ from the cold termination plate, with the electrode-plane normal vector parallel to the magnetic field lines. The cold termination plate is in fact the ionizer that is not used to create plasma. The electrode is either a button electrode $(2.5 \mathrm{~cm}$ diameter) or an annular electrode (inner diameter $1.6 \mathrm{~cm}$, outer diameter $3.5 \mathrm{~cm}$ ). The effect in controlling the ion drift radial profile is similar for both types of electrodes, thus they will be referred to as the electrode. This is the same experimental set-up as in Fig. 1.

The electrode is biased positively with a Hewlett-Packard 6625 dc power supply, which also records the electron current that is drawn. The cold termination plate behind it is also biased (positively, negatively, or at the floating potential) with an Electronic Instruments, Inc. Bipolar Operational Source/Sink 100-1 or the plate can be grounded. The ion (and electron) emitting hot plate/ionizer is always grounded.

LIF parallel measurements reveal one ion Maxwellian-drifting population, as seen in Fig. 22. The ion drift velocity radial profile is controlled in this situation by adjusting the potential difference between the electrode $V_{A}$ and the termination plate $V_{T}$. Most of the 
experiments were performed by keeping $V_{A}$ constant and varying $V_{T}$. The summary of the settings used to control the ion drift radial profile is shown in Table 2.

Shear in the ion parallel velocity can be produced in this configuration when $V_{A^{-}} V_{T} \geq$ $28 \mathrm{~V}$. A typical example of ion parallel drift velocity obtained with the annular electrode is shown in Fig. 23: here, the location of the central depression in the profile corresponds to the location of the electrode.

Remarks:

1) The radial profile of the drift velocity is approximately symmetric.

2) The shear is measured along $x$ axis at $y=0$.

3) The rotational orientation of the hot plate and the effuser were not changed throughout the experiments.

4) Higher magnetic field values $(2 \leq B \leq 2.95 \mathrm{kG})$ were seen to be optimal for SMIC waves destabilization.

5) Typically, the shear layer thickness $L$ extends over a range of $0.8 \mathrm{~cm}<L<1.4 \mathrm{~cm}$. For most of the experimental conditions in which SMIC waves are spontaneously excited, this means that $2.2<L / \rho_{i}<7$ making appropriate the use of the local theory. Moreover, the calculated value of $L_{s}=v_{d i}\left(d v_{d i} / d x\right)^{-1}$ yields typical values in the range $3<L_{s} / \rho_{i}<5$, thus reinforcing the validity of local theory.

An illustration of the control over the radial profile of the ion parallel drift is shown in Fig. 24. Here, Fig.24a represents the radial profile of the parallel ion drift velocity $v_{d i}$ in the case of $V_{A}=15 \mathrm{~V}, V_{T}=0, B=1.5 \mathrm{kG}$. No shear is present for these settings. Symbols in Fig. $24 \mathrm{~b}$ correspond to $v_{d i}$ values when $V_{A}=35 \mathrm{~V}, V_{T}=0, B=2 \mathrm{kG}$ and the annular electrode. Shear is $d v_{d i} / d x=(22.5 \pm 1.4)(\mathrm{ms})^{-1}=(0.16 \pm 0.01) \omega_{c i}$. In Fig.24c, the button electrode is used, with the same settings as in Fig.24b. The large error bars represent plus and minus the ion thermal speed and the small error bars represent the fitting-routine uncertainty in determining $v_{d i}$. A radial profile of shear is shown in Fig. 25.

Shear is produced by the combined effect of the electrode and the termination plate. Specifically, the positive voltage of the electrode slows the ions moving along the magnetic field at radii smaller than the outer radius of the electrode. The ions moving at radii larger than the outer radius of the electrode are influenced by the negative potential of the termination plate and thus have larger drift speed. The proper combination of the voltages 
applied to the electrode and termination plate, respectively, creates the shear in the parallel ion flow.

In some instances, for very large $V_{A}(=50 \mathrm{~V})$ and positive $V_{T}=20 \mathrm{~V}$, a second ion population with very small density counter-streams relative to the initial ion population. The ratio of the two population densities is at least 6:1 (direct streaming versus counter-streaming) and the speed of the second population is roughly half of the direct one, as seen in Fig. 26. Here, the ratio of the densities of the two populations is $6.37: 1$, with $v_{d i l}=580 \mathrm{~m} / \mathrm{s}$ (direct streaming population) and $v_{d i 2}=-220 \mathrm{~m} / \mathrm{s}$ (counter-streaming population). Nevertheless, shear in the direct streaming population is still present, shown in Fig. 27a, while the counterstreaming population has a relatively flat radial profile of the drift velocity, shown in Fig. 27b. For the experiments emphasized here, conditions resulting in two Maxwellian ion populations were avoided. However, experimental conditions for having a single drifting Maxwellian ion population were sought, found and applied.

\section{V.A.2 Characteristics of the shear-modified multi-harmonic ion cyclotron instability}

Spontaneously growing ion cyclotron waves are detected in the presence of shear in the ion parallel flow. A distinctive signature of these waves consists in their multi-harmonic spectra. A number between $n=4$ to $n=11$ spectral features usually can be identified. The correlation between wave parameters, ion flow profiles, electron drift velocities, and plasma boundary conditions is explained in the following.

It is important to mention that throughout these experiments, the plasma density and the plasma potential were uniform over the central plasma column region. Thus, no densitydriven [Hendel and Yamada, 1974] or dc radial electric field induced wave-destabilization mechanisms [Ganguli et al., 1988] are expected to play any role in the experiment except possibly at the plasma-column edge. Drift waves might be destabilized at the edge of the plasma column, where $\nabla n / n$ is large, but such waves were not destabilized during the experiments presented in this dissertation. A typical example of plasma density, measured with a Langmuir probe, is shown in Fig. 28. 
Even in the presence of a biased electrode in the plasma, no significant radial electric field is created. The plasma potential is essentially constant across the plasma column region except at the edge, which is thin (dimension comparable to $\rho_{\mathrm{i}}$. A typical value of the radial electric field in this region is $E_{\mathrm{r}} \sim 10 \mathrm{~V} / \mathrm{m}$ (Fig. 29). Thus, the $\boldsymbol{E} \times \boldsymbol{B}$ ion drift velocity is $v_{E B} \sim 50$ $\mathrm{m} / \mathrm{s}$ for $B=0.2 \mathrm{~T}$, which is much smaller than the ion parallel drift velocity and neither is there significant solid-body rotation in the plasma column. Consequently, no conditions related to the potential relaxation instability [Sato et al., 1977] are met: plasma potential is uniform and, as shown later, no field-aligned electron channel is detected in the plasma, either.

The presentation of the experiments on the effect of shear on ion cyclotron waves growth and propagation begins with the most significant feature, which is the inducement of inverse ion cyclotron damping [Gavrishchaka et al., 2000; Ganguli et al., 2002]. It continues then with showing cases when shear only reduces the ion cyclotron damping and an electron current is needed to support the waves [Gavrishchaka et al., 2000; Ganguli et al., 2002]. The dependence of shear-modified ion cyclotron (SMIC) waves on the magnitude and on the sign of shear, in the presence of small electron drift, is discussed. Finally, the dependence of SMIC waves on the plasma boundary conditions (by imposing restrictions on the parallel wavelength) is discussed. Other particular features of the shear modified multi-harmonic ion cyclotron instability are experimentally measured.

\section{V.A.2.a Inverse ion cyclotron damping induced by ion parallel-velocity shear}

Measurements of the quantities within the ion cyclotron damping factor (54) provide experimental documentation that the factor can be negative, i.e., that ion cyclotron growth can support the waves. Typical values of the damping factor for different spectral features, in a case with intermediate-shear and small $u$, with the parallel-ion-drift velocity radial profile shown in Fig. 23, are: $-0.32 \pm 0.06,-0.26 \pm 0.05,-0.19 \pm 0.04$, and $-0.25 \pm 0.05$ for $n=1$ through $n=4$, respectively. These negative values of the damping factor are based on the measured values of the important parameters presented in Table 3 . Here, $k_{\mathrm{z}} v_{\mathrm{de}}=157 \mathrm{krad} / \mathrm{s}$ and $\omega_{\mathrm{r}} / k_{\mathrm{z}} v_{\mathrm{de}}=0.99$, indicating that inverse electron Landau damping is also present in the waveparticle interaction. A discussion regarding the role of inverse electron Landau damping in this experiment will follow. 
As seen in Fig. 30 where the frequencies of the first three spectral features are recorded versus magnetic field strength, the instability exists in a wide range of magnetic field values. Wave frequencies are smaller than the corresponding ion cyclotron frequency, $\omega_{1 \mathrm{r}} / n \omega_{c i} \sim 0.95$.

Fig. 31 presents the wave spectrum where $n=7$ features are clearly visible within 1.5 orders of magnitude. The $\boldsymbol{k}$ vector components are determined based on the fitting shown in Fig. 32 where the solid black line is a fitted sinusoid plotted based on the values of $k_{z}$ and $k_{y}$ stated above. The dotted and dashed sinusoids are curves plotted using the values of $k_{z}$ given by the uncertainty limits. To reinforce the determination of the finite value of $k_{z}$, the circle method is shown in Fig. 33 using the same data from Fig. 32. The circle method consists of plotting the probe-tip relative phase difference as a function of probe orientation using the polar coordinate as the probe angle with the magnetic field and the radial coordinate as the probe-tip relative phase difference. An offset of $120^{\circ}$ is chosen for clarity. The wavevector components are the coordinates of the circle center. The fitted values are the values previously found: $k_{z}=0.024 \mathrm{~cm}^{-1}$ and $k_{y}=-2.120 \mathrm{~cm}^{-1}$.

In this instability, the theory-predicted mechanism of inverse ion cyclotron damping, which uses the local approximation, can be simply understood from the growth rate formula (57). In the absence of shear, the ion cyclotron damping term is always equal to unity,

$$
1-\left[k_{y} d v_{d i} / d x /\left(k_{z} \omega_{c i}\right)\right]\left(1-n \omega_{c i} / \omega_{1 r}\right)=1 \text { if } d v_{d i} / d x=0,
$$

leading to the well-known ion cyclotron damping. In the presence of small or intermediate shear, as well as large or intermediate $u$, the damping factor can decrease,

$$
0<1-\left[k_{y} d v_{d i} / d x /\left(k_{z} \omega_{c i}\right)\right]\left(1-n \omega_{c i} / \omega_{1 r}\right)<1,
$$

meaning that ion cyclotron damping can be reduced. This will have significant ramifications on the wave, as shown later. As it passes through zero, the damping factor changes sign and becomes negative,

$$
\text { 1- }\left[k_{y} d v_{d i} / d x /\left(k_{z} \omega_{c i}\right)\right]\left(1-n \omega_{c i} / \omega_{1 r}\right)<0 \text {, }
$$


as was measured in the experiment, thus leading to inverse ion cyclotron damping, a way through which ion cyclotron waves can grow unstable even in the absence of inverse electron Landau damping.

Another way of looking at the change of the ion cyclotron damping factor sign, from the non-local point of view, is to recall the ion-cyclotron-damping resonance condition

$$
\omega_{r}-k_{z} v_{d i}=n \omega_{c i}(n= \pm 1, \pm 2, \ldots)
$$

which is the ion-Landau-damping resonance condition $\left(\omega_{1-}-k_{z} v_{d i}=0\right)$ for $n \neq 0$. In the no-shear case, meaning $v_{d i}=$ constant, it follows that for $n=1$, all ions with the parallel velocity equal to $\widetilde{v}_{\phi \mathrm{z}}$, the gyration-frame wave phase velocity,

$$
v_{d i}^{r}=\frac{\omega_{r}-\omega_{c i}}{k_{z}}=\widetilde{v}_{\phi \mathrm{z}}
$$

will interact resonantly with the EIC wave. The number of resonant ions is the same for all locations across the plasma column in this case.

In the case of shear in the ion parallel velocity,

$$
v_{d i}(x)=v_{d i 0}+\frac{d v_{d i}}{d x} \Delta x
$$

the ion cyclotron resonance condition becomes

$$
\omega_{r}-k_{z} v_{d i 0}-k_{z} \frac{d v_{d i}}{d x} \Delta x=\omega_{c i},(n=1)
$$

where $v_{\mathrm{di} 0}$ is the ion parallel drift velocity at position $x=0$. This spatial dependence of the resonance condition makes it possible that the local ion drift velocity $v_{\mathrm{di}}(x)$ is larger or smaller than the wave phase velocity $\widetilde{v}_{\phi \mathrm{z}}=\left(\omega_{r}-\omega_{c i}\right) / k_{z}$

$$
\widetilde{v}_{\phi \mathrm{z}}<\left(v_{d i 0}+\frac{d v_{d i}}{d x} \Delta x\right)=v_{d i}(x)
$$

or

$$
\widetilde{v}_{\phi \mathrm{z}}>\left(v_{d i 0}+\frac{d v_{d i}}{d x} \Delta x\right)=v_{d i}(x) .
$$

In the case of plasmas with parallel-velocity shear, the number of resonant ions depends on the spatial position $x$. Therefore, the total number of resonant ions can be either smaller, equal or larger than the number of resonant ions in a plasma with zero-shear. Ion cyclotron damping depends directly on the number of resonant ions. Therefore, the case when 
the total number of resonant ions is smaller in an inhomogeneous-ion-flow plasma than in a homogeneous-ion-flow plasma is related to the decrease in the ion cyclotron damping. Similarly, the case when the total number of resonant ions is larger in an inhomogeneous-ionflow plasma comparing to a homogeneous-ion-flow plasma relates to the enhancement of the ion cyclotron damping.

To illustrate this concept, Fig. 34 shows the LIF-measured parallel ion velocity distribution across the plasma column (various $x$ positions). The straight line represents the wave phase velocity $\widetilde{v}_{\phi z}=480 \mathrm{~m} / \mathrm{s}$. It can be seen that all ions propagating along $z$ at radial distances $-1<x<1$ cm have $v_{\mathrm{di}}(x)<\widetilde{v}_{\phi \mathrm{z}}$. But for ions outside this column, $v_{\mathrm{di}}(x)>\widetilde{v}_{\phi \mathrm{z}}$.

The experimental conditions did not prevent an electron current $\left(v_{d e}=68 v_{t i}=37 \mathrm{~km} / \mathrm{s}\right)$ from being present in the plasma. The wave propagates parallel to the current, $k_{z} v_{d e}=107$ $\mathrm{krad} / \mathrm{s}>0$. However, by itself, this current could not destabilize these multi-harmonic ion cyclotron wave. Recalling the CDEIC waves formula (49) for electron drift necessary to excite waves in homogeneous-ion-flow plasmas, with the experimental parameters given and $T_{i}=0.25 \mathrm{eV}, T_{e}=0.26 \mathrm{eV}$, it follows that the electron current necessary for destabilizing the fundamental would be in this case $\left(v_{\mathrm{de}}\right)_{\text {crit }} \cong 65 \mathrm{~km} / \mathrm{s}=120 v_{\mathrm{ti}}$. To excite the higher harmonics which are observed to have comparable values of $k_{z}$, a current directly proportional to $\omega_{1 \mathrm{r}} \sim n \omega_{c i}$ is necessary [Ganguli et al., 2002]. It can be thus inferred that in this case the shear provides the most significant source of free energy for the wave growth, but the wave still taps the available free energy in the drifting electron population.

The absence of electron velocity shear means that the parallel ion shear in the electron frame $\left(d v_{\mathrm{diz}} / d x\right)_{\mathrm{e}}$, the electron shear in the ion frame $\left(d v_{\mathrm{dez}} / d x\right)_{\mathrm{i}}$, and the ion shear in the lab frame $d v_{\mathrm{diz}} / d x$ have identical absolute values. Also, electron drift is not enhanced by the positive voltage applied to the electrode and no field-aligned electron-current channel is formed in the plasma. The electron drift is uniform and extends to the full extent of the plasma column as shown in Fig. 35.

\section{Regimes}

It is possible to present the effect that shear has in destabilizing ion cyclotron waves using a simple picture based on the wave parameters (frequency and wavevector components). Fig. 36 shows the three possible regimes: inverse ion cyclotron damping, reduced ion 
cyclotron damping and enhanced damping for a shear $\frac{d v_{d i}}{d x}=19.3(\mathrm{~ms})^{-1}=0.14 \omega_{\mathrm{i} i}$. The three points represent actual data points obtained in those regimes, one in the inverse ion cyclotron damping regime and the other two in the reduced ion cyclotron damping regime. As expected, no waves were detected when ion cyclotron damping was enhanced by shear, even when the threshold predicted by the homogeneous-plasma theory was exceeded, as explained later.

\section{V.A.2.b Multi-harmonic ion cyclotron waves dependence on shear magnitude and sign i. Shear-magnitude dependence}

The experiment is performed using the north ionizer and the annular electrode placed at an axial distance of $275 \mathrm{~cm}$ from it. The magnetic field direction is from the north ionizer toward the south ionizer. Thus, both ions and electrons propagate in the $+z$ direction. To measure the dependence of multi-harmonic ion cyclotron waves on shear magnitude, the shear sign is incremented from negligible to small and intermediate values.

First, the case of negligible shear is discussed. The applied voltage to the electrode is $V_{\mathrm{A}}=15 \mathrm{~V}$ and the termination plate is grounded, $V_{T}=0$. A negligible shear is created, as seen in Fig. 24a which shows the ion parallel-velocity radial profile. Shear is $d v_{d i} / d x \leq 1(\mathrm{~ms})^{-1}$ $\sim 0.01 \omega_{c i}(B=0.15 \mathrm{~T})$. The electron drift is set to $v_{d e}=48 \mathrm{~km} / \mathrm{s}=100 v_{t i}$. No waves are excited in this case, since the ion cyclotron damping is not sufficiently reduced so that a current smaller than the homogeneous-plasma threshold $\left(v_{\mathrm{de}}\right)_{\text {crit }} \cong 60 \mathrm{~km} / \mathrm{s}=120 v_{\mathrm{ti}}$ could destabilize the waves.

A step towards increasing the shear is taken using the annular electrode biased at $V_{\mathrm{A}}=35 \mathrm{~V}$ and the termination plate grounded, so a shear with the magnitude $d v_{d i} / d x=(17.3 \pm 2.1)$ $(\mathrm{ms})^{-1}$ is created. This corresponds to $d v_{d i} / d x=(0.10 \pm 0.02) \omega_{c i}$. The ion cyclotron wave created in this case has a fundamental angular frequency $\omega_{\mathrm{r}}=164.1 \mathrm{krad} / \mathrm{s}$ with $k_{z}=(0.04 \pm 0.005) \mathrm{cm}^{-1}$ and $k_{y}=(-2.71 \pm 0.01) \mathrm{cm}^{-1}$. The ion drift is parallel to the magnetic field direction and $v_{\mathrm{d} 0}=450$ $\mathrm{m} / \mathrm{s}, B=0.25 \mathrm{~T}$. Thus, the wave frequency in the ion frame is

$$
\omega_{1 r}=\omega_{r}-k_{z} v_{d 0}=(164.1-1.8) \mathrm{krad} / \mathrm{s}=162.3 \mathrm{krad} / \mathrm{s} \text {. }
$$

Then 


$$
1-\frac{\omega_{c i}}{\omega_{1 r}}=1-\frac{172.7 \pm 2.4}{162.3 \pm 3.0}=1-(1.064 \pm 0.003)=-0.064 \pm 0.003
$$

The damping factor is in this case $\left\{1-\left[k_{y} d v_{d i} / d x /\left(k_{z} \omega_{c i}\right)\right]\left(1-n \omega_{c i} / \omega_{r}\right)\right\}=0.61 \pm 0.12$ which is smaller than unity. The measured number of wave harmonics is $n=8$ and the electron drift is $v_{d e}=100 v_{t i}$.

Maintaining the same magnetic field and increasing the shear by applying $V_{\mathrm{A}}=35 \mathrm{~V}$ to the electrode and $V_{\mathrm{T}}=-5 \mathrm{~V}$ creates an increased shear, $d v_{d i} / d x=(22.4 \pm 4.2)(\mathrm{ms})^{-1}=$ $(0.13 \pm 0.03) \omega_{\mathrm{i}}$ and the ion drift is practically the same, $v_{d 0}=460 \mathrm{~m} / \mathrm{s}$. The wave frequency is now $\omega_{1}=(160 \pm 3.0) \mathrm{krad} / \mathrm{s}$ with the wavevector components $k_{z}=(0.045 \pm 0.005) \mathrm{cm}^{-1}$ and $k_{y}=(-$ $2.92 \pm 0.01) \mathrm{cm}^{-1}$. The damping factor is now $\left\{1-\left[k_{y} d v_{d i} / d x /\left(k_{z} \omega_{c i}\right)\right]\left(1-n \omega_{c i} / \omega_{1 r}\right)\right\}=0.41 \pm 0.16$. The number of wave harmonics increases to $n=10$ and the electron drift is $v_{d e}=80 v_{t i}$.

The damping factor is not negative, thus shear cannot sustain by itself the wave growth. Inverse electron Landau damping is necessary in this situation. Measurements of electron current reveal that $v_{d e}=80-100 v_{t i}$ in the cases presented above. This value is much below the excitation threshold of CDEIC waves in homogeneous-ion-flow barium plasma, $\left(v_{\mathrm{de}}\right)_{\text {crit }} \cong 60 \mathrm{~km} / \mathrm{s}\left(=120 v_{\mathrm{ti}}\right)$, consistent with the prediction $\left(\left(v_{\mathrm{de}}\right)_{\text {crit }}=150 v_{\mathrm{ti}}\right)$ of Drummond and Rosenbluth [1962] for the current-driven electrostatic ion-cyclotron (CDEIC) instability. This threshold corresponds to a balance between inverse electron Landau damping and ioncyclotron damping. Since the damping is reduced by the presence of free energy provided by the inhomogeneous ion flow, a smaller electron current is required for sustaining the wave growth. This reduced excitation threshold is expected since the presence of the parallelvelocity shear decreases ion-cyclotron damping (or eliminates the damping entirely, as described earlier in this section) for the fundamental and the harmonics, thereby requiring less inverse electron Landau damping to achieve the above-mentioned balance.

Another experimental finding on the shear-magnitude dependence of SMIC waves is the correspondence between shear magnitude and wave fundamental frequency. The mode is detected on a Langmuir probe inserted in the shear layer region (at $x=-1.7 \mathrm{~cm}$ ). The shear magnitude is varied incrementally by applying various bias voltages to the termination plate, as described before. The mode fundamental frequency varies with the shear as seen in Fig. 37.

For negligible shear there is little mode destabilization. As shear increases, the mode is increasingly destabilized. At larger, meaning intermediate, values of shear, the mode 
frequency is far from $\omega_{c i}$ and its amplitude has increased. This is consistent with the fact that the damping is large when $\omega_{1 r}=n \omega_{\mathrm{ci}}[$ Stix, 1996] and the ion-cyclotron damping factor is equal to unity [Ganguli et al., 2002].

\section{ii. Shear-sign dependence}

To reverse the sign of the shear, the magnetic field direction is reversed, so that the field lines now point towards the north ionizer. Applying a bias voltage of $V_{A}=35 \mathrm{~V}$ to the annular electrode, a shear equal to $d v_{d i} / d x=-17.3(\mathrm{~ms})^{-1}=-0.1 \omega_{c i}$ is now created. The change in shear sign is due to the change in ion parallel velocity sign from $v_{d i}=450 \mathrm{~m} / \mathrm{s}$ to $v_{d i}=-450$ $\mathrm{m} / \mathrm{s}$ because the direction of the $z$-axis has reversed with the reversed magnetic field direction. By mirroring the coordinate system such that the $x$-axis maintains its original direction and the $y$-axis reverses sign, the shear sign is also reversed. In this situation

$$
1-\left[k_{y} d v_{d i} / d x /\left(k_{z} \omega_{c i}\right)\right]\left(1-n \omega_{c i} / \omega_{1 r}\right)>1
$$

and increasing the shear actually increases the ion cyclotron damping factor. Experimentally, there are no waves detected in this case even if the electron current is much larger, i.e., $v_{d e}=$ $150 v_{t i}=-72 \mathrm{~km} / \mathrm{s}$ and the electron drift is anti-parallel to the magnetic field direction than in all correct-sign shear cases. This value of electron drift is higher than the measured threshold for destabilizing EIC waves in homogeneous WVU-Q-machine plasma, which is $\left(v_{d e}\right)_{c r i t}=$ $\pm 120 v_{t i}= \pm 60 \mathrm{~km} / \mathrm{s}$. These experimental values were found while performing the experiments described in this dissertation, when single-feature, current-driven EIC waves were destabilized.

It can thus be concluded that reversing the sign of the shear leads to increasing the ion cyclotron damping and even a larger electron drift cannot overcome the damping increase. This is compelling evidence that shear plays an essential role in the wave stability and it can affect wave-particle interaction. No cases when $k_{\mathrm{z}} / k_{\mathrm{y}}$ changed spontaneously sign were found in these experimental conditions.

If waves were driven solely by electron current and no shear was present in the ion flow, they could be destabilized by the large current applied and they would propagate anti- 
parallel to the magnetic field such that $k_{z}<0$ and $k_{z} v_{d e}>0$. The fact this is not the case in the experiment provides additional support for the fact that shear is essential to the wave growth.

To further test the experimental observations on shear-sign dependence another set of measurements was performed. The magnetic field was set to point toward the south ionizer which was heated up to emit ions and electrons. The electrode was placed $275 \mathrm{~cm}$ away from the south ionizer, while the north ionizer was kept cold (as a termination plate). In this configuration, a slightly smaller, negative, shear was created, $d v_{d i} / d x=-13.7(\mathrm{~ms})^{-1} \sim-0.08 \omega_{c i}$. Nevertheless, waves could not be destabilized even in the presence of an electron current $v_{d e}=100 v_{t i}$, providing supporting evidence for the experimental findings presented above.

\section{V.A.2.c Multi-harmonic shear-modified ion cyclotron waves dependence on plasma boundary conditions}

\section{i. Wave azimuthal propagation}

Plasma boundary conditions were adjusted to determine the dependence the number of SMIC wave harmonics on the perpendicular wavevector $k_{y}$. The annular electrode is biased at $V_{A}=45 \mathrm{~V}$ and the termination plate is grounded. A shear of $d v_{d i} / d x=(24.2 \pm 3.6)(\mathrm{ms})^{-1}=$ $=(0.14 \pm 0.025) \omega_{c i}$ is created $(B=0.25 \mathrm{~T})$. The multi-harmonic SMIC waves created have a spectrum consisting of at least $n=4$ harmonics. Measuring the wavevector components at a radial location $r=1.6 \mathrm{~cm}$ yields $k_{z}=0.05 \mathrm{~cm}^{-1}$ and $k_{y}=-1.71 \mathrm{~cm}^{-1}$. Since the ion temperature is $T_{i y}=0.25 \mathrm{eV}$, the ion gyroradius is $\rho_{i}=0.3 \mathrm{~cm}$ and $\left(k_{y} \rho_{i}\right)^{2} \sim 0.26$. Decreasing the magnetic field from $B=0.25 \mathrm{~T}$ to $B=0.2 \mathrm{~T}$ and applying $V_{A}=41 \mathrm{~V}$ leads to a slight increase in the shear to $d v_{d i} / d x=(0.15 \pm 0.027) \omega_{c i}$ and an increase in the ion gyroradius to $\rho_{i}=0.38 \mathrm{~cm}$. The multiharmonic wave detected in these conditions has a spectrum consisting of at least $n=7$ harmonics and the wavevector components $k_{z}=0.15 \mathrm{~cm}^{-1}$ and $k_{y}=-2.12 \mathrm{~cm}^{-1}$. This increase in the number of harmonics correlates to the increase in $\left(k_{y} \rho_{i}\right)^{2} \sim 0.65$. With the wavevector components reported in section V.A.2.bi discussing the SMIC waves dependence on shear magnitude, which are $k_{z}=0.04 \mathrm{~cm}^{-1}$ and $k_{y}=-2.92 \mathrm{~cm}^{-1}$, the parameter $b=\left(k_{y} \rho_{i}\right)^{2}=\left(-2.92 \mathrm{~cm}^{-1} \mathrm{x}\right.$ $0.3 \mathrm{~cm})^{2} \approx 0.8$ is correlated with the presence of $n=10$ harmonics in the spectrum, reinforcing the conclusion that the number of harmonics increases with increasing $b$. 
As predicted by the model, an increase in the number of harmonics is measured when $b=\left(k_{y} \rho_{i}\right)^{2}$ increases. For a more accurate documentation of the model predictions, more experimental data points are needed. Difficulties in measuring accurately the wavevector components while maintaining a fixed shear magnitude prevented the presentation in this dissertation of more data points. The slight increase in shear (by 7\%) leads one to surmise that the increase in shear is less significant compared to the increase in $\left(k_{y} \rho_{i}\right)^{2}$ which is significant (by $300 \%$ ) as is the number of harmonics (from 4 to 10 ).

The azimuthal propagation is characterized by the azimuthal mode number $m=\left|k_{y} r\right| \sim 3$. Measuring the wavevector components at a different radial position $(r=-0.6 \mathrm{~cm})$ yields $m=\mid-$ $4.97 \mathrm{~cm}^{-1} \times(-0.6 \mathrm{~cm}) \mid \approx 3$, in agreement with the previous measurement. Thus, the mode propagation is given by $e^{i 3 \theta}$, where $\theta=\theta_{0}+\omega_{r}$. The fact that the azimuthal mode number does not have actual integer values could be explained by the slight radial asymmetry in the shear layer. From Fig. 25 it can be seen that the average-shear layer position is between $-2 \mathrm{~cm}$ and $0.8 \mathrm{~cm}$ and between 0 and $0.9 \mathrm{~cm}$, respectively.

According to the model, the multi-harmonic spectrum requires not only parallelvelocity shear but also a value of $\left(k_{\mathrm{y}} \rho_{i}\right)^{2}$ comparable to unity, with the number of harmonics increasing with increasing $\left(k_{\mathrm{y}} \rho_{i}\right)^{2}$. From Fig. 31, the number of harmonics within 1.5 orders of magnitude is one for the no-shear case and many more than one for the shear cases. For negligible shear, the damping factor is near unity and only a single dominant spectral features is observed, as shown in Fig. 31a, and $\left(k_{\mathrm{y}} \rho_{\mathrm{i}}\right)^{2}=(0.4 \pm 0.1)$. For more than an order-ofmagnitude larger shear $\left(d v_{\mathrm{di}} / d x=0.12 \omega_{\mathrm{ci}}\right)$ and an increase to $\left(k_{\mathrm{y}} \rho_{\mathrm{i}}\right)^{2}=(0.94 \pm 0.1)$, the waves exhibit spectral features at eleven ion-cyclotron harmonics within the same 1.5 orders of signal magnitude, as shown in Fig. $31 \mathrm{~b}(\mathrm{i})$ and Fig. 31b(ii). The simultaneous appearance of multiple harmonics is expected since the critical value of the parallel-velocity shear for destabilizing the $n$th harmonic at a given value of $v_{\text {de }}$ becomes independent of harmonic number $n$ for large values of $\left(k_{y} \rho_{i}\right)^{2}$. Fig. 31b(i) shows that this harmonic structure exists both when the fundamental is below the ion-cyclotron frequency and Fig. 31b(ii) shows the harmonic structure when the fundamental is above the ion-cyclotron frequency.

When the 2.5-cm-diameter, circular button electrode is substituted for the annular electrode, a similar spectrum is observed, as shown in Fig. 24c, but at smaller mode amplitude. This could be explained by the fact that a larger electrode permits the exitence of 
waves with a larger range of values of $b$. In this manner, the waves have the possibility of selecting the optimal $b$ for growth, given the plasma parameters magnetic field, shear magnitude, electron drift, and ion and electron temperature.

The linear nature of the wave destabilization mechanism can be seen from the information contained in Fig. 38. By plotting the wave normalized frequency versus the harmonic number, it can be seen that $\left(\omega_{1 r}-n \omega_{\mathrm{ci}}\right) / \omega_{\mathrm{ci}}$ is independent of $n$ for the first seven harmonics. For the rest of the harmonics, the uncertainty in finding the spectral peak is larger than for the first seven. Comparison with predicted wave frequency predicted by the linear model (open circles, from Gavrishchaka et al., [2000]), in the case when the waves are destabilized by inverse ion-cyclotron damping, shows the similarity between the experimental data and the theoretical predictions. If the wave harmonics were not each harmonics of the ion cyclotron frequency but of the wave fundamental frequency, they should be found along the central oblique line in Fig. 38. It represents the normalized frequencies for which $\omega=$ $n \omega_{\text {tundamental }}$. The outermost lines represent the uncertainty in finding $\omega_{\text {fundamental }}$ multiplied by $n$.

The error bars shown in Fig. 38 represent the normalized frequency width at threequarters of the maximum spectral power. The fact that the errror bars for the first seven harmonics stay outside the region defined by the two outermost oblique lines, which indicate $\omega=n\left(\omega_{\text {tundamental }} \pm \delta \omega_{\text {fund }}\right)$, reinforces the experimental observation of an independent destabilization mechanism for each of the wave harmonics.

\section{ii. Wave longitudinal propagation}

The wave longitudinal wavevector component $k_{z}$ can be varied by adjusting the plasma column length. SMIC waves have long parallel wavelengths, sometimes almost as long as the plasma column length which is $3 \mathrm{~m}$. One of the smallest values of the wavevector parallel component is $k_{\mathrm{z}}=2.4 \mathrm{~m}^{-1}$, and it corresponds to the wavelength parallel component $\lambda_{\mathrm{z}}=2 \pi / k_{\mathrm{z}}=2.6 \mathrm{~m}$. To change the plasma length, the electrode is placed at a closer position, 30 $\mathrm{cm}$ from the north ionizer. Both the ion and the electron flows are parallel to the magnetic field ( $B=0.25 \mathrm{~T}$ ) since only the north plasma source is used, therefore both ions are electrons are created by this source only. With the electrode biased at $V_{A}=40 \mathrm{~V}$ and the termination plate at $V_{T}=-2 \mathrm{~V}$, a shear of $d v_{d i} / d x=20.1(\mathrm{~ms})^{-1} \sim 0.12 \omega_{c i}$ is created. The electron current is 
$v_{d e}=75 v_{t i}$. The SMIC waves are not destabilized in this configuration, though. An analysis of the ion cyclotron damping factor shows that it is larger than in the case when the plasma column was 10 times longer. Indeed, the minimum value of the parallel wavevector is now $k_{z}$ $\sim 2 \pi / \lambda_{z} \sim 21 \mathrm{~m}^{-1}$, a value approximately 10 times larger that the values obtained in the long-

plasma-column case $\left(k_{z} \sim 2.4 \mathrm{~m}^{-1}\right)$. This implies that, considering the same $k_{y}$ as in the longplasma case, the damping factor would also be much larger, since the factor depends on $\left(k_{z}\right)^{-1}$. Hence, ion cyclotron damping cannot be sufficiently reduced such that a subcritical electron drift could destabilize the waves.

The longitudinal extent of the plasma and the longitudinal boundary conditions play important roles in SMIC waves destabilization. It is through longitudinal boundary conditions that the shear is created in the ion parallel (=longitudinal) flow. Aside from this, this experiment shows the destabilization dependence on wave parallel wavelength. This indicates that multi-harmonic SMIC waves destabilization is more probable in those cases when plasma has a large axial extent, as in the case of space plasma where multi-harmonic ion-cyclotron waves were reported in the upper $\mathrm{F}$ region of the ionosphere [Ergun et al., 1998].

The electron drift values $v_{d e}=25-100 v_{t i}$ generally measured in this experiment - when multi-harmonic waves are present and the damping factor is $0<1-\left[k_{y} d v_{d i} / d x /\left(k_{z} \omega_{c i}\right)\right](1-$ $\left.n \omega_{c i} / \omega_{1 r}\right)<1$-cannot sustain by themselves any of the waves detected, irrespective of the number of harmonics seen. The critical drift to destabilize the fundamental is, as mentioned before, $\left(v_{d e}\right)_{c r i t}=120 v_{t i}$. Hence, the presence of multi-harmonic spectra can be attributed to the presence of shear and proper plasma boundary conditions.

\section{V.A.2.d Correlation between wave frequency and wave propagation characteristics}

A unique signature of the shear-modified multi-harmonic ion cyclotron waves destabilization mechanism, predicted by theory, is experimentally verified. According to the model, in order for the ion cyclotron damping to be reduced, the quantity $k_{y} d v_{d i} / d x /\left(k_{z} \omega_{c i}\right)(1-$ $\left.n \omega_{c i} / \omega_{1 r}\right)$ must be positive. This imposes a certain correlation between the signs of shear $d v_{d i} / d x$, wavevector components ratio $k_{y} / k_{z}$ and wave angular frequency in the ion frame $\omega$. For instance, in the case of a positive shear $d v_{d i} / d x>0$ and negative ratio $k_{y} / k_{z}<0$, the wave 
frequency can only be smaller than the ion gyrofrequency $\omega_{c i}$. Other combinations are also possible, according to the model.

The experimental observation of this signature shows that wave frequency changes from $\omega_{1 r} / \omega_{c i}=0.920 \pm 0.003$, when $k_{y} / k_{z}<0$, to $\omega_{1 r} / \omega_{c i}=1.050 \pm 0.003$ when $k_{y} / k_{z}>0$, while the shear is fixed. The measured parameters $d v_{d i} / d x=23.3(\mathrm{~ms})^{-1} \sim 0.15 \omega_{c i}, k_{z}=0.04 \mathrm{~cm}^{-1}, k_{y}=-1.91$ $\mathrm{cm}^{-1}$ and $\omega_{1 r}=\omega_{\mathrm{r}}-k_{z} v_{d i}=24.01 \mathrm{krad} / \mathrm{s}-4 \mathrm{~m}^{-1} \mathrm{x} 495 \mathrm{~m} / \mathrm{s}=22.7 \mathrm{krad} / \mathrm{s}=0.92 \omega_{c i}$, show that the ion cyclotron damping factor is smaller than unity, $1-\left[k_{y} d v_{d i} / d x /\left(k_{z} \omega_{c i}\right)\right]\left(1-\omega_{c i} / \omega_{1 r}\right)=0.56$. Inserting a floating electrostatic probe into the plasma column at $z=92 \mathrm{~cm}$ from the ionizer changes the plasma boundary conditions and leads to the mode reversing azimuthal propagation direction from $k_{\mathrm{y}}=-1.91 \mathrm{~cm}^{-1}$ to $k_{y}=2.67 \mathrm{~cm}^{-1}$. This change in the wave propagation would preserve the value of the ion cyclotron damping factor and thus maintain the wave growth. To maintain maximum growth, the wave preserves $k_{z}$, since an increase would lower the growth. After the wave changes the azimuthal propagation, the ion cyclotron damping factor remains smaller than unity, $1-\left[k_{y} d v_{d i} / d x /\left(k_{z} \omega_{c i}\right)\right]\left(1-\omega_{c i} / \omega_{1 r}\right)=0.53$.

\section{V.A.2.e Waveform, wave spectrum and $k$-spectrum analysis}

Waveform analysis of multi-harmonic ion cyclotron waves generated in the presence of shear in the ion flow reveals the spiky nature of the time series (Fig. 39, bold line). The time series is in sharp contrast with the sinusoidal waveform of the single-feature CDEIC wave destabilized in a plasma with negligible parallel-velocity shear and shown in Fig. 39 and represented by the thin line. The time-domain-fluctuations of SMIC waves generated experimentally in the $\mathrm{Q}$ machine present strong resemblance with the fluctuations of the parallel electric field seen both in space and in simulations [Gavrishchaka et al., 2000; Ganguli et al., 2002], presented in Fig. 40.

The mode $\boldsymbol{k}$-spectrum is analyzed for the case of inverse ion cyclotron damping, case when $k_{z}$ has minimum values. With the two-tip probe oriented anti-parallel to the magnetic field $\left(\theta=-180^{\circ}\right)$, the measurement yields $-k_{z}$. Fig. 41a presents the wave spectrum (around the fundamental frequency) for one of the data sets, i.e. for one of the 120 acquistions. Fig. $41 \mathrm{~b}$ depicts the mode axial structure with $\left|k_{z}\right|=0.026 \mathrm{~cm}^{-1}$. This is consistent with the finite $k_{z}$ value presented earlier in the case that inverse ion cyclotron damping occurs. The maximum power 
is associated with the spectral feature at $26.48 \mathrm{kHz}$, for which $\Delta \Phi=-0.09 \mathrm{rad}$. Since the distance between the probe tips is $3.5 \mathrm{~mm}$, then $\left|k_{z}\right|=0.026 \mathrm{~cm}^{-1}$ for this feature. The $\boldsymbol{k}$ spectra for higher harmonics yield a similarly peaked lineshape and values of axial and azimuthal wavevector components similar to those of the fundamental harmonic.

To determine the azimuthal wavevector structure, the probe is now placed such that the plane of the two tips is at $\theta=90^{\circ}$ to the magnetic field. The measurement yields now the wavevector azimuthal component $k_{y}=-2.21 \mathrm{~cm}^{-1}$. Again, the measurements reveal that the instability has only one eigenmode, as shown in Fig. 42.

As presented in Table 3, the ion cyclotron damping factor is negative for the first four harmonics of the wave. This is because the values of the wavevector parallel component $k_{\mathrm{z}}$ are approximately the same for all four wave-frequency harmonics. Also, the wavevector perpendicular component values $k_{\mathrm{y}}$ are approximately identical for $n=1$ to $n=4$. This fact also supports the idea that the wave harmonics are independently destabilized by inverse ion cyclotron damping.

\section{V.A.2.f Mode amplitude radial profile}

Measurements of the mode amplitude radial profile are not easy to perform due to plasma perturbations associated with the probes inserted in the region where the mode is localized. Nevertheless, some indication of the spatially averaged mode amplitude in the region corresponding to the cylinder having the base defined by the electrode is obtained from the mode amplitude detected with the electrode itself. This voltage perturbation is converted into current perturbation, knowing the impedances of the circuit elements involved in the measurement. The current perturbation is an indication of the plasma density perturbation, $\delta I / I \sim \delta n / n$. Current perturbation is normalized knowing the measured electron current drawn by the electrode. Measurements of mode amplitude in this region show that it can be as high as $\delta n / n \sim 10 \%$ over the area of the button electrode ( $1.2 \mathrm{~cm}$ radius).

In the outer shear-layer region the mode amplitude is detected by the method described above, with the only difference that an electrostatic probe is used. Fig. 43 shows that there is negligible mode amplitude far away from the shear layers $(x= \pm 3 \mathrm{~cm})$, but the mode amplitude increases at the shear layer $(\delta n / n \sim 4 \%$ at $x=-1.6 \mathrm{~cm})$. 


\section{V.B Shear-modified ion acoustic waves}

\section{V.B.1 Control of the ion-parallel-velocity drift radial profile}

The Q-machine is set up in the Double Ionizer No Electrode (DINE) configuration. There are no electrodes inserted in the plasma (except for the electrostatic probes). Either one or both atomic ovens' heating power is turned on. This means that ions were produced either at both ends of the Q machine or only at one end. In either case, electrons are produced at both ends. A single drifting-Maxwellian ion population is obtained either by using one ionizer or by using both ionizers and biasing negatively enough one of them such that the ions produced are trapped in the negative sheath region.

The hot plates are biased with respect of each other. The applied voltage is negative or zero. Combinations of applied biases and hot-plate powers can enable control on the ion parallel-drift radial profile. For instance, applying $V_{n}=-10 \mathrm{~V}$ to the north hot plate and $V_{s}=-30$ $\mathrm{V}$ to the south hot plate, with the ions coming from the north hot plate (along the magnetic field lines), a velocity shear of $d v_{d i} / d x=-0.48 \omega_{c i}$ is created at $0.9 \mathrm{~cm}$ from the plasma column center. Setting $V_{n}=V_{s}=0 \mathrm{~V}$ but using only the north-end atomic oven, a shear of $d v_{d i} / d x=0.21 \omega_{c i}$ is created. Some of the settings used to control the ion drift radial profile are summarized in Table 4. For all the settings used in Table 4, the magnetic field points from south to north.

Shear in the ion parallel velocity is usually obtained when ions are created simultaneously at both end of the Q machine. The power emitted at the ionizers differs by approximately $20 \%$. The ionizer producing less power is biased at a larger (in absolute value) negative voltage. Typical values are $-30 \mathrm{~V}$ for the ionizer emitting less power and $-10 \mathrm{~V}$ for the one emitting more power. When ions are produced at only one end of the Q machine, the ionizer is grounded while the other end of the machine (emitting just electrons) is typically biased at -5 to $-10 \mathrm{~V}$.

In either case, the shear magnitude is controlled by the difference between the applied bias voltages. Increased parallel-velocity shear correlates with increased bias-voltage difference, as seen in Table 4. Although no systematic determination of the dependence of 
shear magnitude on the relative bias was performed, it can be stated that the relative-bias threshold necessary for creating shear was $|\Delta V|=5 \pm 1 \mathrm{~V}$. The shear magnitude increased with increasing $|\Delta V|$ and a saturation in the maximum value of shear appeared when $|\Delta V|=25 \pm 2 \mathrm{~V}$. The different values of the relative bias are obtained by maintaining the lowest (in absolute value) applied bias while varying the other one.

The sign of the shear can be switched from positive to negative while maintaining the direction of the magnetic field (northward) and the direction of the ion flow (northward). Thus, for the case of grounded south ionizer (i.e., ions are produced at the south end) and grounded north-end termination plate, the shear created is positive. Applying a negative bias voltage to the termination plate $(-5 \mathrm{~V}$ to $-10 \mathrm{~V})$ makes the shear change sign from positive to negative. This change in the shear sign is due to the ion parallel-velocity at small radii becoming larger than the parallel-velocity of ions at large radii. On the other hand, when ions were produced at both ends without the termination plate, the change in the sign of parallelvelocity shear is obtained by switching the applied bias voltages. Thus, the direction ion parallel-velocity is reversed. Since both the magnetic field direction and the magnitudes in the ion velocity profile are still maintained, the shear sign changes because the sign of $\Delta v_{\mathrm{di}}$ reverses.

A more complicated issue is why the shear layer usually appeared at radial positions between 0.7 and $0.9 \mathrm{~cm}$ from the plasma-column center. No obvious correlation with other plasma or boundary conditions discontinuities was found. A two-dimensional scan of the parallel-velocity radial profile shows that one half-cylinder of plasma can have a different range of parallel ion velocities than the other half-cylinder [E. Reynolds, private communication]. It is believed that this two-dimensional shear profile is responsible for the cylindrically symmetric shear-driven waves.

Creating ions either at both ends of the Q-machine (using both the south and the north atomic ovens) or at one end only (by only using one atomic oven) creates shear with the same magnitude and scale sizes. In Fig. 44 (a) is shown a set of raw LIF data (before the Zeemansplit removal) in the case of both atomic ovens on and in Fig. 44 (b) is presented the case when only the north oven is on. The biases are the same in both cases, $V_{n}=-5 \mathrm{~V}$ and $V_{s}=-25 \mathrm{~V}$. In Fig. 44 (a) the laser beam is swept from positive to negative values of $x$; in Fig. 44 (b) it is swept the other way. The two lineshapes look identical. 
The control is obtained under the circumstance that the two hot plates present domains on the rhenium-coated surface. Although attempts were made to apply a uniform rheniumcoating, the existence of a non-uniform coating cannot be ruled out. Domains are visible on the rhenium layer surface on both hot plates, each consisting of patches of different color tint. However, no clear connection between the position of the shear layer and the positions of the domanins on the rhenium-coating surface could be established. Thus, it is less likely that the inhomogeneity in the ion parallel-velocity can be attributed solely to the inhomogeneous rhenium-coating.

Several remarks can be made here:

1) Lower magnetic field ( $1 \leq B \leq 1.5 \mathrm{kG})$ might favor producing the shear. These low $B$ values were seen to be proper for SMIA waves destabilization.

2) The shear is measured along the $x$ axis at $y=0$.

3) Shear created in the DINE configuration is not cylindrically symmetric, the shear layer that appears at $e . g ., x=0.9 \mathrm{~cm}$ does not appear at $x=-0.9 \mathrm{~cm}$.

4) The position of the hot plate with respect to the $Q$ machine is the same in all experiments, i.e., it was not rotated.

5) Shear created using the south-end hot plate is smaller than the one created using the north end plate by almost a factor of two.

6) Typically, the shear layer thickness $L$ extends over a range of $0.5 \mathrm{~cm}<L<1.1 \mathrm{~cm}$. For most of the experimental conditions in which SMIA waves are spontaneously excited $1.5<L / \rho_{i}<3$, making appropriate the use of the weak shear limit theory. Moreover, the calculated value of $L_{s}=v_{d i}\left(d v_{d i} / d x\right)^{-1}$ yields typical values in the range $3<L_{s} / \rho_{i}<5$, thus reinforcing the weak shear theory.

The plasma density radial profile is constant over the plasma column region where the density is large, with values around $n=10^{9} \mathrm{~cm}^{-3}$. Negligible electric fields were observed in the region of the plasma column. As shown later in this chapter, they did not exceed typically 20 $\mathrm{V} / \mathrm{m}$.

Applying large negative biases on the hot plates, the electrons can be thermally insulated from the hot plates [Motley, 1975]. Therefore the electron temperature can be higher than the hot plate temperature. The experiments presented in this work also show increased electron temperature when large negative potentials are applied to the hot plates. The onedimensional energy theory [von Goeler and Motley, 1970] predicts than in electron rich 
plasmas, the ion temperature can be larger than the hot-plate temperature. Moreover, direct LIF measurements show that perpendicular ion temperature is also larger than the hot plate temperature [Hirschberg et al., 1967; Hinnov et al., 1969; Brown, 1972] and almost equal to the electron temperature [Teodorescu et al., 2002a], an effect believed to be caused by the thermal insulation of the ions from the hot plate. Thus, the effect of the large negative biases applied to the hot plates could be the thermal insulation of both species (electrons and ions) from the hot plate. The ion parallel temperature is decreased comparing to the perpendicular ion temperature due to the acceleration process occurring when the ions pass through the sheath but is still higher than the hot plate temperature. This ion acceleration would be no longer an isothermal process, as in the case of grounded or weakly biased hot plates, but an adiabatic process. Given this different type of ion acceleration and the effect of the other biased hot plate (both setting the plasma boundary conditions), ions can have different perpendicular drift velocities.

The electron depletion of the negatively biased hot plate sheath affects not only the plasma potential but also the ion dynamics in the sheath. Motley [1975] shows that isotherms on the hot plate surfaces can vary by as much as $\pm 150{ }^{\circ} \mathrm{C}$. Hill [1983] presents evidence from LIF measurements that ion parallel drift velocity depends on hot plate temperature. Thus, the various combinations of local hot plate temperatures and ion-rich sheath potentials could lead to the creation of shear in the ion parallel drift velocity.

Another way of understanding the possibility that shear is created in this experimental configuration comes from recalling that of all the barium atoms that hit the rhenium-coated hot plate surface, only $12 \%$ are ionized (at a hot plate temperature $T=2200 \mathrm{~K}$ ) according to Saha's equation. The remaining neutral atoms are present in the sheath region and form the majority of the heavy species (ions and atoms). The local neutrals pressure could easily exceed (by an order of magnitude) the pressure determined by the ionization gauge, since the gauge is positioned far away from the sheath region at a position where almost all the barium atoms have already been absorbed on the chamber walls. Recall that the recombination probability of barium atoms with the chamber cold walls is approximately $99 \%$. The neutral pressure in the sheath region can be larger than expected from $12 \%$ ionization at the hot plate because not all the atoms emitted by the barium oven actually hit the hot plate due to the geometry of the effuser. Based on the ion saturation current measurements of plasma density and on the measured background pressure $\left(3 \times 10^{-6}\right.$ Torr $)$, the atomic barium density in the 
sheath could be as high as $10^{-4}$ Torr. This large value of neutral pressure matches that cited by Skiff and Fasoli [1993] in connection with their experimental work on turbulent transport in which parallel velocity shear was present. They mention that parallel shear could only be induced when xenon atoms where introduced in the vacuum chamber at pressures around $10^{-4}$ Torr. They could not induce shear when introducing lighter-mass neutrals at the same pressure range. Since xenon and barium have very close atomic masses (131 and 138, respectively), it is possible that the role played by xenon in inducing parallel shear in Skiff's and Fasoli's experiment is played by barium in the experiment presented in this dissertation. It is not clear, based on the mechanism described in their paper, the direction in which the parallel velocity would change with radius in the experiments presented in this dissertation.

Willig et al. [1997] and later Agrimson et al. [2001] reported a different way of controlling the direction of the parallel ion flow with respect to the magnetic field direction in their experiments aimed at producing parallel-velocity shear. In both works, experiments were performed in double-ended Q machines, ions and electrons being produced at both ends. Ion flow direction was controlled by creating an imbalance between the powers emitted at the two ionizers with ions drifting from the ionizer emitting the most power towards the other one. Indirect measurements of ion drift velocity are cited to confirm the flow control.

\section{V.B.2 Characteristics of the shear-modified ion acoustic instability}

Spontaneously growing ion acoustic waves are seen in barium plasma in the presence of inhomogeneous ion parallel flow and homogeneous parallel electron drift. Here, both ends of the Q machine operate with $P_{s}=2.15 \mathrm{~A} \times 1.46 \mathrm{kV}, P_{n}=2.20 \mathrm{~A} \times 1.40 \mathrm{kV}$ and $B=0.1 \mathrm{~T}$. Also, both atomic ovens operate. The ions produced at the south-end hot plate are trapped by applying $V_{s}=-25 \mathrm{~V}$ to the plate. The north hot plate is grounded.

A typical ion distribution LIF measurement is shown in Fig. 44(a). This represents a set of 25 LIF measurements in which the laser beam points along the magnetic field. At each $x$ position the ion distribution is measured. The laser beam is swept over the entire plasma cross-section in increments of $2.5 \mathrm{~mm}$. This increment size is chosen since the width of the beam itself is $2 \mathrm{~mm}$. The spectral position of the hollow-cathode lamp is $\lambda_{0}=585.5301 \mathrm{~nm}$ in 
this coordinate system, yielding $v_{d i}=0$. A sample of the fitting routine and its outcome (the ion velocity distribution) are shown in Fig. 45. The ion drift velocity radial profile determined from the LIF-measurement set is shown in Fig. 46. The shear in the ion drift velocity is $(-38.2 \pm 6.3)(\mathrm{ms})^{-1}$, representing $(-0.55 \pm 0.09) \omega_{\mathrm{ci}}$. The error bars in determining $v_{d i}$ are associated with the errors in the fitting routine. The parallel ion-temperature is $T_{i z}=0.19 \mathrm{eV}$ here while Langmuir-probe measuremnets show that the electron temperature is $T_{e}=0.34 \mathrm{eV}$.

The electron drift radial profile measured with the single-sided Langmuir probe indicates a homogeneous electron flow across the plasma column at $v_{d e}=-37.7 \mathrm{~km} / \mathrm{s}=-82 v_{t i}$ (Fig. 47). The small $\left(v_{\mathrm{de}}=0.15 v_{\mathrm{te}}\right)$ net electron flow (electrons are a Maxwellian population, Fig. 48) is from the south-end hot plate toward the north-end hot plate. The radial homogeneity in the electron parallel drift means that the ion shear in the electron frame $\left(d v_{\mathrm{di}} / d x\right)_{\mathrm{e}}$, the electron shear in the ion frame $\left(d v_{\mathrm{de}} / d x\right)_{\mathrm{i}}$, and the ion shear in the lab frame $d v_{\mathrm{di}} / d x$ have identical absolute values.

Fig. 49 shows the ion density radial profile based upon the ion saturation current collected by an electrostatic probe. The plasma potential determined with an emissive probe is shown in Fig. 50. The maximum value of the radial dc electric field is $\left|E_{r \text { max }}\right|=17 \mathrm{~V} / \mathrm{m}$ which is considered small for influencing the wave-particle dynamics.

The wave detected in these plasma conditions has a low $\left(\omega<\omega_{c i}\right)$, magnetic field independent frequency $f=1.9 \mathrm{kHz}$. Doppler-shifted wave frequencies $\left(\omega_{\mathrm{r}}=\omega_{\mathrm{lr}}+k_{\mathrm{z}} v_{\mathrm{di}}\right)$ are shown in Fig.51(a) where $k_{z} v_{d i}=-22 \mathrm{~m}^{-1} \cdot 510 \mathrm{~m} / \mathrm{s}=-11.2 \mathrm{krad} / \mathrm{s}\left(=-0.11 \omega_{c i}\right)$ and in Fig.51(b) where $k_{z} v_{d i}=13 \mathrm{~m}^{-1} \cdot 480 \mathrm{~m} / \mathrm{s}=6.3 \mathrm{krad} / \mathrm{s}\left(=0.07 \omega_{c i}\right)$. Waves are detected for a range of magnetic field strengths between 0.9 and $1.9 \mathrm{kG}$, shown in Fig.52.

The wave bandwidth is given by $\Delta f / f \sim 15 \%$. The wavevector components are determined from the fitted sinusoid shown in Fig. 53 which yields $k_{z}=-0.507 \mathrm{~cm}^{-1}$ and $k_{y}=-$ $0.331 \mathrm{~cm}^{-1}$. The measurement is made with the two-tip probe positioned at $r=-2.3 \mathrm{~cm}$. Thus the radial propagation mode number is $m=\left|k_{y} r\right| \sim 1$. The wave propagation direction is mostly along the magnetic field, at an angle

$$
\theta=\tan ^{-1}\left(k_{y} / k_{z}\right)=33^{0} .
$$

A two dimensional wave phase-difference measurement also provides information on the mode azimuthal propagation characteristics. One probe is fixed (reference) and the other one sweeps across the plasma column, measuring $\Delta \Phi=k \Delta r=k_{y} \Delta y$. The distance between the 
tips of the two probes is $\Delta z=22 \pm 1 \mathrm{~cm}$, depending on the $y$ position of the movable probe tip. The position of the fixed probe tip is given by the coordinates $(1 \mathrm{~cm}, 1 \mathrm{~cm})$ while the movable probe tip has positions at each corner of the squares in the mesh. The spatial structure of the phase difference, shown in Fig. 9, reveals a peak and a trough separated by $180^{\circ}$, a clear indication of the $m=1$ azimuthal propagation mode. A possible explanation for the fact that the phase difference radial structure is not symmetric can be provided by the fact that the shear is not symmetric. Since shear is an additional source of free energy for the wave, any asymmetry in its radial position could appear as asymmetry in the wave phase difference radial structure.

The $k_{y}$ spectrum (taken at $270^{\circ}$ ) shown in Fig. 54 indicates that the instability has one azimuthal eigenmode. Measurements of azimuthal wavevector component $k_{y}$ performed at various radial positions $x$ support the $m=1$ mode azimuthal propagation characteristic. Fig. 55 shows the correlation between $k_{y}$ and the radial position $r$ (here, $y=0$ in all measurements, so $r$ $=x$ ). The solid lines represent $m=k_{y} r=0,1$ and 2 , respectively.

Data analysis shows that this wave has the dispersion relation of a shear-modified ionacoustic wave. The wave propagates in the direction of the electron flow, i.e., $k_{z} v_{d \mathrm{~d}}=6.27 \mathrm{x}$ $10^{2} \mathrm{krad} / \mathrm{s}>0$, and oppositely to the ion flow: $k_{z} v_{d i z}=-7.14 \mathrm{krad} / \mathrm{s}<0$ (at the shear location). The wave frequency in the ion frame is then

$$
\omega_{1 r}=\omega_{r}-k_{z} v_{d i, z}=19.07 \mathrm{krad} / \mathrm{s}=0.25 \omega_{\mathrm{ii}}
$$

The ion sound speed along the magnetic field is

$$
c_{s z}=\sqrt{\frac{k_{B}\left(T_{e}+2 T_{i z}\right)}{m_{i}}}=925 \mathrm{~m} / \mathrm{s} .
$$

In this formula, the value of the ion adiabatic exponent $\gamma_{0}$, which multiplies the ion temperature, is $\gamma_{0}=2$ (from fluid theory, other possible values are 1 or 3 ). This value is also used by Mikhailovskii [1992] to show that the dispersion relation of "ion acoustic KelvinHelmholtz waves" [Mikhailovskii, 1992] in the presence of inhomogeneous parallel ion flow has identical forms when derived using either the fluid model or the kinetic model.

The wave parallel phase velocity in the ion frame is given by

$$
v_{\phi z}=\frac{\omega_{1 r}}{k_{z}}=1084 \mathrm{~m} / \mathrm{s}
$$


and is higher than the ion sound speed

$$
v_{\phi z}>c_{s z} .
$$

To connect with the model, shear and wavevector measurements are used to determine $\sigma$. Here, $k_{z} / k_{y}=1.64$ and the shear normalized to $\omega_{c i}$ is

$$
\frac{d v_{d i} / d x}{\omega_{c i}}=-\frac{38.3(\mathrm{~ms})^{-1}}{69.1 \mathrm{krad} / \mathrm{s}}=-0.55
$$

Then

$$
\sigma=\sqrt{1-\frac{-0.55}{1.64}}=1.15>1
$$

Thus,

$$
\sigma c_{s z}=1.15 \times 925 \mathrm{~m} / \mathrm{s}=1064 \mathrm{~m} / \mathrm{s} \approx 1084 \mathrm{~m} / \mathrm{s}=\frac{\omega_{1 r}}{k_{z}} .
$$

Hence,

$$
\omega_{1 r} \approx \sigma k_{z} c_{s z} \text {. }
$$

This good match between the measured and the calculated values of the wave phase velocity parallel component can be found for all ion acoustic waves destabilized in the presence of parallel-velocity shear.

Other important information regarding the nature of this wave is given by the fact the wave is destabilized by a current below the predicted threshold value in homogeneous plasma [Kindel and Kennel, 1971]. For a homogeneous-flow barium plasma with the electron and ion temperatures found in this experiment, the homogeneous theory [Kindel and Kennel, 1971] predicts a threshold current (105)

$$
\left(v_{d e}^{c r i t}\right)_{C D E I A} \approx 300 v_{t i} .
$$

Since it is found in the electron drift measurements that $v_{d e}=82 v_{t i}$, it means that the wave is driven unstable by a much smaller electron drift than in a homogeneous plasma,

$$
\left(v_{d e}\right)_{S M I A}<\left(v_{d e}^{c r i t}\right)_{C D E I A} .
$$


This result is in accord with the theoretical predictions of the model [Gavrishchaka et al., 1998].

The shift in the SMIA wave parallel phase velocity and the associated decrease in the ion Landau damping can be determined directly by means of LIF measurements. In Fig. 56 is shown the parallel-velocity ion distribution in the ion frame, $v_{d i}=460 \mathrm{~m} / \mathrm{s}$, the ion sound speed $c_{\mathrm{sz}}$, the shifted wave phase velocity $\sigma c_{\mathrm{sz}}$ and the measured wave phase velocity $\omega_{I r} / k_{\mathrm{z}}$. The reduction in ion Landau damping associated with this up-shift is shown in Fig. 56b. The derivative of the velocity distribution is considered proportional to the ion Landau damping as shown by Chen [1983] and Stix [1996].

Ion perpendicular drift velocity has a homogeneous profile. Measurements are made along $y$ direction at various $x$ positions. For comparison, the parallel and perpendicular drift velocity radial profiles are shown in Fig. 57. The corresponding shears (i.e., $d v_{d z} / d x$ and $d v_{d y} / d x$, respectively) are $-0.55 \omega_{c i}$ and $\pm 0.04 \omega_{c i}$, respectively, meaning that shear in the perpendicular ion drift velocity is negligible. The radial profiles of the two shears are presented in Fig. 58.

\section{V.B.2.a SMIA waves dependence on ion parallel-velocity shear sign}

The dependence of SMIA waves on shear has been determined by reversing the sign of the shear. Grounding both hot plates and using only the north atomic beam, a positive shear

$$
\frac{d v_{d i}}{d x}=41.3(\mathrm{~ms})^{-1}=0.46 \omega_{c i}
$$

is created. In this case, for $u=k_{z} / k_{y} \geq 1$,

$$
0<\sigma=\sqrt{1-\frac{0.46}{u}}<1
$$


which means that the wave parallel phase velocity is downshifted to values smaller than the ion sound speed. In this case the ion Landau damping is strong and the SMIA wave is damped A case of positive shear associated with no waves is shown in Fig. 59.

It is also possible that $u=k_{z} / k_{y}<<1$, case in which

$$
\sigma^{2}=1-\frac{0.46}{u}<0
$$

In this situation the wave no longer exhibits its resonant behavior, but becomes a purely growing mode with $\omega_{1 r}=0$. This is the D'Angelo wave which is a wave stationary in the ion frame. This wave has also been observed in these experiments and will be discussed later.

\section{V.B.2.b SMIA waves dependence on ion parallel-velocity shear magnitude}

Increasing the shear magnitude by increasing the negative bias increases the wave frequency, decreases the wave propagation angle and increases the wave growth rate. These results are shown in Fig. 60. An increase in the electron drift speed and electron temperature is also detected when increasing the negative bias applied. The increase in negative shear leads to increased wave frequency and thus, to a larger up-shift in the wave phase velocity. This leads in turn to a further decrease in ion Landau damping which can account for the increase in the wave growth rate. The simultaneous increase in the electron drift speed observed can also contribute to the overall wave growth. Electron drift $\mathrm{v}_{\mathrm{de}}$ increases from $v_{d e}=65 v_{t i}$ up to $v_{d e}=150 v_{t i}$.

\section{i. SMIA waves growth rate dependence on shear}

To determine the SMIA wave growth rate, one hot plate bias is stepped from a value corresponding to a subcritical electron drift speed to a value corresponding to a supercritical electron drift speed and the absolute (temporal) growth of SMIA waves amplitude is monitored. To determine the dependence of SMIA wave growth rate on shear magnitude, this growth rate measurement is performed for three different step sizes, each associated with a different value of final shear and final electron drift speed. The stepped-biased is applied to 
the north hot plate while the south hot plate is grounded. The increasing wave amplitude is detected with an electrostatic probe and recorded with the waveform digitizer. Fig. 61(a,b) shows the growth of SMIA waves for a stepped-bias $V_{n}=-10 \mathrm{~V}$.

To determine the wave growth rate, it is necessary to remove the initial pulse of the waveform, which occurs as the plasma recovers from the applied stepped-bias. The same plasma response to stepped biases is mentioned by Correll et al. [1975] and by Carroll [1997]. To analyze the waveform, a method consisting in fitting the pulse with a combination of exponential and polynomial functions is used. Fig. 61a (bottom) and Fig. 61b (top) show the fitting curve and the extracted waveform, obtained by subtracting the fitting curve form the pulsed waveform. The fitting curve has the expression $y(t)=240+700 \times e^{-0.0023 t}$, where $t$ is the time.

To determine the exponential fit of the extracted waveform, the square of the relative waveform amplitude is first plotted on a semi-logarithmic scale, natural logarithm of the relative amplitude vs. time in Fig. $61 \mathrm{~b}$ (bottom). The exponential fit is then determined by fitting a straight line of equation $y(t)=2 \gamma\left(t-t_{0}\right)$, where $y(t)=\ln \left[a(t) / a\left(t_{0}\right)\right]$ and $a(t)$ is the wave amplititude at time $t$, whose slope is twice the growth rate, through the waveform oscillation peaks represented here by the cross symbols. The constant is determined using the least square method. This fitted value yields the growth rate $\gamma / \omega_{c i}=(4.4 \pm 2.1) \times 10^{-3}$. A more detailed discussion on the determination of SMIA wave growth rate is presented in Appendix 6.

\section{ii. SMIA waves propagation angle dependence on shear}

Also associated with increasing shear and increasing wave real frequency are increasing values of $u=k_{z} / k_{y}$. Since the wave propagation angle is defined as

$$
\theta=\tan ^{-1}\left(\frac{k_{y}}{k_{z}}\right)
$$

this means that increased values of $u$ correspond to decreased values of $\theta$. In the graph of Fig. 60 , the three symbols represent three different values of $u$, i.e., $u=0.7$ (square), $u=1.4$ (circle) and $u=1.9$ (triangle), respectively. The larger values of $u$ are associated with larger values of shear $\left(d v_{d i} / d x\right) \omega_{c i}^{-1}$, because the combination of large shear and large $u$ can maintain $\sigma>1$, the necessary condition for SMIA waves destabilization. For a given shear magnitude, too large a 
$u$ can lead to $\sigma<1$, case in which the waves are damped. The relation between shear magnitude and wave propagation angle can be understood by taking the ion temperature ratio $T_{i y} / T_{i z}$, i.e., the temperature anisotropy, into account. This dependence will be addressed later.

\section{V.B.2.c SMIA waves propagation direction}

SMIA waves always propagate in the direction of the electron flow, irrespective of the direction of the ion flow. Control over the wave propagation direction is thus obtained by controlling the electron flow. This is done by controlling the negative bias to the hot plates and by controlling the hot plates' power. For approximately equal hot-plate powers, with the magnetic field pointing from north to south, Table 5 summarizes the observed wave propagation direction when adjusting the applied voltages on the hot plates. Ions are only created at the north hot plate and their flow direction is from north to south in both cases discussed here (Fig. 62 and Table 5).

For a given set of biases applied to the hot plates, wave propagation direction can be controlled by varying the hot plate powers (Fig. 63 and Table 6). Both ionizers produce ions in this configuration and there is one drifting-Maxwellian ion population.

In summary, when both hot plates radiate the same power, the direction of the electron flow and consequently, wave propagation direction, is from the most negatively biased plate toward the least negatively biased plate. If a small negative bias is applied to one of the two hot plates, the electron flow direction can be also controlled by the power radiated at each plate: the flow is from the most radiative plate to the least radiative one. Electrons form a single drifting-Maxwellian population.

\section{V.B.3 SMIA waves in anisotropic plasma}

The experiments presented in this work on SMIA waves were performed in an anisotropic barium-plasma. While all the basic features of the isotropic model (wave phase velocity upshift, wave growth rate increase with increasing shear, wave propagation angle decrease with decreasing shear) were documented, important wave characteristics, not accounted for by the initial model [Gavrishchaka et al., 1998, 1999] but discussed by 
Spangler [2001] and Spangler et al. [2002] were found to be present only in the presence of anisotropic plasma. Among these are the decrease in the wave propagation angle and the increase in the growth rate with increasing ion temperature anisotropy $T_{i y} / T_{i z}$. The theoretical aspect of this behavior was studied by Spangler [2001] and Spangler et al. [2002].

\section{V.2.3.a Control over ion temperature anisotropy in a $\mathbf{Q}$ machine}

Various degrees of ion temperature anisotropy can be produced in the barium plasma created in the Q machine. Control over the anisotropy is achieved by applying different negative biases on each of the two hot plates. Each hot plate is set to radiate the same power. In this case, the plasma becomes electron rich and it can become thermally insulated from the hot plates. Motley [1975] describes that in such case electron temperature may become larger than the hot plate temperature. Early experiments by Hinnov et al. [1963] and Hirschberg et al. [1966] in Q-machine-produced barium plasma showed an increase in the ion perpendicular temperature compared to the hot plates temperature as a function of plasma density. They measure the ion temperature with a swept Fabry-Perot interferometer and document the dependence of the ratio between ion and hot plate temperatures on plasma density. For plasma density much smaller than the critical value (defined as the value for which the plasma potential is zero), i.e., $n_{0} / n_{c} \sim 10^{-2}$, they showed that the ion temperature can be as high as twice the hot plate temperature. In the experiments on the SMIA wave dependence on anisotropy presented in this dissertation, the density is around $n_{0} \sim 10^{8} \mathrm{~cm}^{-3}$; since for barium $n_{c} \sim 10^{10}$ $\mathrm{cm}^{-3}$, the fact that perpendicular ion temperature can be 2.1 times larger that the hot plate temperature is in agreement with experiments by Brown [1972] and with the one-dimensional energy theory [von Goeler and Motley, 1970].

Thus, by creating an electron-rich plasma, the temperatures of both ions and electrons can be elevated. Due to the parallel acceleration of the ions in the sheath, they gain energy in the parallel direction. This leads to a decrease in the ion parallel temperature compared with the ion perpendicular temperature, $T_{i z}<T_{i y}$. The electron and ion perpendicular temperatures are approximately equal, $T_{i y} \sim T_{e}$. 
Fig. 64 depicts a typical radial profile of parallel $T_{i z}$ and perpendicular $T_{i y}$ ion temperatures, measured at a distance $z=46 \mathrm{~cm}$ from the north hot plate. There is no evidence of structures in any of the two radial profiles.

\section{V.B.3.b The effect of ion temperature anisotropy in the growth and propagation of SMIA waves}

The primary role of anisotropy in these experiments is reflected in the trend of decreasing propagation angle with increasing ion-temperature anisotropy, making the $k_{z} / k_{y}$ values not arbitrary and not anisotropy-independent. Whereas the optimum value of $k_{\mathrm{z}} / k_{\mathrm{y}}$ is relatively insensitive to the values of $v_{\mathrm{de}}$ and $d v_{\mathrm{diz}} / d x$ in the isotropic model, being usually $k_{\mathrm{z}} / k_{\mathrm{y}}<<1$ for $d v_{\mathrm{diz}} / d x<\omega_{c i}$, the experimentally observed optimum values of $k_{\mathrm{z}} / k_{\mathrm{y}}$ vary considerably with $d v_{\mathrm{diz}} / d x$ (from $k_{\mathrm{z}} / k_{\mathrm{y}}<1$ to $k_{\mathrm{z}} / k_{\mathrm{y}}>1$ ) and are strongly correlated with the anisotropy.

This distinction is documented in Fig. 65. The value of $\sigma$ associated with observed SMIA waves in plasma with $T_{\mathrm{i}} \approx T_{\mathrm{e}}$ is expected to be equal to or larger than the value (larger than unity) associated with the SMIA-wave excitation threshold. If SMIA waves were to remain unstable as the parallel-velocity shear increases, one would expect either $k_{\mathrm{z}} / k_{\mathrm{y}}$ or $\sigma$ to increase also, as is apparent in the predictions in Fig. 65 for the isotropic case. The exaggerated degree to which $k_{\mathrm{z}} / k_{\mathrm{y}}$ increases with shear in the experiment can be attributed to the fact that anisotropy is increasing along with the shear and that $k_{\mathrm{z}} / k_{\mathrm{y}}$ is correlated with anisotropy. This correlation is not expected for ion-acoustic waves propagating exactly parallel or exactly perpendicular to the magnetic field. This is consistent with the theoretical predictions of Spangler [2001] and Spangler et al., [2002]. A more detailed discussion on their results is presented in Chapter VI.

Fig. 66 shows the dependence of the measured growth rate (filled triangle, diamond, and square) and real frequency (filled circles) on ion-temperature anisotropy. The qualitative trend of increasing growth rate can be accounted for without anisotropy by just changing $v_{\text {de }}$ and $d v_{\mathrm{diz}} / d x$, if allowed to select arbitrary and anisotropy-independent values of $k_{z} / k_{y}=0.11$, open triangle; 0.12 , open diamond; 0.12 , open square, and holding both ion temperature and electron temperature to be equal and isotropic, as shown in the isotropic-model's predictions 
that replicate the trend of increasing growth rate for experimentally matched values of $v_{\mathrm{de}}$ and $d v_{\mathrm{diz}} / d x$.

Fig. 67 shows how the parallel-velocity shear shifts both the parallel and perpendicular wave phase velocities $\left(\omega_{\mathrm{r} r} / k_{\mathrm{z}}, \omega_{\mathrm{rr}} / k_{\mathrm{y}}\right.$, respectively $)$ in the ion frame $\left(\omega_{\mathrm{r}}=\omega_{\mathrm{r}}-k_{\mathrm{z}} v_{\mathrm{diz}}\right)$ by the factor $\sigma$ from the ion sound speed $c_{\mathrm{sz}}\left(=\sqrt{k_{B}\left(T_{e}+2 T_{i z}\right) / m_{i}}\right)$ and $k_{\mathrm{z}} c_{\mathrm{sz}} / k_{\mathrm{y}}$, respectively, to $\sigma c_{\mathrm{sz}}$ and $\sigma k_{\mathrm{z}} c_{\mathrm{sz}} / k_{\mathrm{y}}$, respectively, where $m_{i}$ is the ion mass. This significantly reduces the effect of parallel and perpendicular ion Landau damping on the waves relative to the electron Landau growth from the parallel electron drift. The ion-frame phase velocity in the presence of shear corresponds to small slope for both the parallel and perpendicular ion velocity distributions in Fig. 67a (i.e., small magnitude for the derivatives in Fig. $67 \mathrm{~b}$ of the parallel and perpendicular ion velocity distributions), as expected for undamped waves. The decrease in the magneticfield-aligned Landau damping is responsible for the wave overcoming the competition between inverse electron Landau damping and ion Landau damping whereas the decrease in perpendicular Landau damping is linked to the optimum value of ion-temperature anisotropy for a particular wave propagation angle. A correlation between large values of $k_{\mathrm{z}} / k_{\mathrm{y}}(=1.5)$ and large values of $T_{i y} / T_{i z}(=1.8)$ measured in the experiment is observed. This can be understood simply by requiring the wave velocities in the two directions to exceed by the same percentage the ion thermal speeds associated with the two directions. This two-dimensional requirement leads to the relationship $k_{\mathrm{z}} / k_{\mathrm{y}} \propto T_{i y} / T_{i z}$ for undamped modes propagating at small angles $\left(k_{\mathrm{z}} / k_{\mathrm{y}}>1\right)$ to the magnetic field, as should be expected for oblique propagation.

Fig. 68a shows the observed dashed-line correlation $k_{\mathrm{z}} / k_{\mathrm{y}} \propto T_{i y} / T_{i z}$. Fig. $68 \mathrm{~b}$ depicts this correlation in terms of the relationship, solid line, taken directly from the dashed line, between the wave propagation angle $\theta=\tan ^{-1}\left(k_{\mathrm{y}} / k_{\mathrm{z}}\right)$ and the ion-temperature anisotropy. These results, clearly showing that smaller propagation angles are associated with larger values of anisotropy, support the description of the role of anisotropy on wave-propagation angle. According to this simple picture, waves propagating at angles inconsistent with the $k_{\mathrm{z}} / k_{\mathrm{y}} \propto T_{i y} / T_{i z}$ relationship would experience significant damping, supporting the hypothesis that anisotropy significant influences the ion-acoustic-wave growth rate.

The parameters $T_{\mathrm{e}}, T_{\mathrm{iy}}, T_{\mathrm{iz}}, \omega_{\mathrm{i}}, d v_{\mathrm{di}} / d x, k_{\mathrm{y}}, k_{\mathrm{z}}, \omega_{l r}, \gamma$ associated with the three experimental points in Fig. 66 can be found in Table 7. Increases in $T_{\mathrm{e}}, T_{\mathrm{iy}}$, and $k_{\mathrm{z}}$ result in an 
increase in $\omega_{\mathrm{lr}}=k_{\mathrm{z}} \sigma c_{\mathrm{sz}}$. The $8 \%$ change in $T_{\mathrm{iy}} / T_{\mathrm{e}}$ is negligible but the $33 \%$ change in $T_{\mathrm{iz}} / T_{\mathrm{e}}$ is nonnegligible. The observed anisotropy is interpreted to be a necessary condition for parallel propagating SMIA waves rather than a result of wave-induced ion energization, since no significant localized ion heating is observed.

If $k_{\mathrm{y}}=0$, velocity shear would not influence the ion-acoustic waves and temperature anisotropy would not influence the wave propagation angle. In this experiment, SMIA waves having nonzero $k_{\mathrm{y}}$ were excited by producing parallel-velocity shear. Once $k_{\mathrm{y}} \neq 0$, the influence of ion-temperature anisotropy on the growth and propagation of the ion-acoustic waves became apparent.

As mentioned before, no structure in the radial profile of ion-temperature is observed, indicating that no significant localized ion heating results of these ion-acoustic waves. Ioncyclotron waves, in contrast, induce significant localized ion heating [Rynn et al., 1974; Correll et al., 1977; Dakin et al., 1976, Stern et al., 1976; 1981; Hatakeyama et al., 1977; Koepke et al., 1998; Amatucci et al., 1998]. Ion energization by ion-acoustic waves is not expected unless the ion drifts exceed by more than an order of magnitude the ion thermal speed [Vaivads et al., 1998]. Based on the observations of the unstructured radial profile of ion temperature, the very Maxwellian ion velocity distributions, and lack of fluctuations with spectral features outside the ion-acoustic-wave range, it can be concluded that the observed anisotropy does not arise from the presence of any wave mode.

Computer-program results, obtained by Scime et al. [2002] using the above-mentioned experimental parameters of SMIA waves in anisotropic barium-plasma, show good agreement with the experimental results. Unstable waves are only found if $T_{i y} / T_{i z}>1$ and their growth rate increases with increasing shear (Fig. 69). If all experimental parameters, except ion temperature anisotropy which is set to unity, were used in the computer-code, no unstable SMIA waves would be found. The symbols represent $k_{z} / k_{y}=0.7$ (triangle), 1.4 (diamond) and 1.9 (square), respectively. In Fig. 70, the corresponding growth rates are negative, whereas they become positive as shown in Fig. 69 if $T_{i y} / T_{i z}>1$.

\section{V.C. The D'Angelo instability}

The D'Angelo instability is a purely growing instability in the ion frame. The instability is driven by inhomogeneous parallel ion flow and does not require electron drift for 
growing. Thus, this wave will always propagate in the direction of ion flow, irrespective of the direction of electron flow. This behavior is in contrast with SMIA waves which propagate in the direction of the electron drift irrespective of the ion flow direction. Another distinction between the two modes is given by the sign of

$$
\sigma^{2}=1-\frac{k_{y}}{k_{z}} \frac{d v_{d i} / d x}{\omega_{c i}}
$$

which is positive for the SMIA instability and negative for the D'Angelo instability, as shown by Gavrishchaka et al. [1999].

The D'Angelo instability has been obtained in these experiments by using the Q machine in the DINE configuration. The north ionizer was biased at $V_{N}=-4 \mathrm{~V}$ and the south one at $V_{S}=10 \mathrm{~V}$. The ion drift was $v_{d i}=-1050 \mathrm{~m} / \mathrm{s}$. A positive shear in the single driftingMaxwellian ion population (from south toward north, i.e., negative ion drift since the magnetic field $B=0.1 \mathrm{~T}$ points southward) of $d v_{d i} / d x=15.9(\mathrm{~ms})^{-1} \sim 0.23 \omega_{c i}$ was obtained. The observed wave has a broad spectrum $(0.5 \mathrm{kHz}<f<3 \mathrm{kHz})$ peaking at $1.2 \mathrm{kHz}$. Wavevector measurements show that $k_{z}=-0.07 \mathrm{~cm}^{-1}$ and $k_{y}=-0.75 \mathrm{~cm}^{-1}$, meaning that

$$
\sigma^{2}=1-\frac{-0.75}{-0.07} \frac{0.23 \omega_{c i}}{\omega_{c i}}=-1.46 .
$$

The mode propagates parallel to the ion flow. The electron drift detected, $v_{d e} \sim 0.001 v_{t e}$, cannot support either SMIA, ion acoustic or ion cyclotron waves. The lab-measured, Doppler-shifted wave angular frequency $\omega_{r}=7530 \mathrm{rad} / \mathrm{s}$ (calculated at the peak frequency) matches the measured value of $k_{z} v_{d i}=-7 \mathrm{~m}^{-1} \times(-1050 \mathrm{~m} / \mathrm{s})=7350 \mathrm{rad} / \mathrm{s}$, indicating that the mode real frequency in the ion frame is $\omega_{l \mathrm{r}}=\omega_{r}-k_{z} v_{d i} \approx 0$. Thus, this is a purely growing mode in the ion frame. Wave frequency is insensitive to the magnetic field strength in the range of $B=0.1-0.2$ $\mathrm{T}$ but the wave is stabilized outside this range.

As seen in Fig. 71, the mode maximum amplitude is sharply localized at the shear layer, but smaller plasma density perturbations are seen as far as $1 \mathrm{~cm}$ from the shear layer. Fig. 72 presents the lab-frame measured wave spectrum. The wave frequency spectrum is very broad, as described in the literature, with $\Delta \omega=0.6 \omega_{\mathrm{ci}}$ or $\Delta \omega \omega 600 \%$ within 1.5 orders of magnitude. Wavevector measurements are made at $x=0.8 \pm 0.02 \mathrm{~cm}, z=69 \mathrm{~cm}$. 
Parallel electron drift measurements document a homogeneous radial profile that extends to the plasma column edges. Electron flow is detected in both parallel and antiparallel directions, but the ratio of the two electron fluxes (parallel and antiparalel) is $j_{e} u p / j_{e}$ down $\approx 1.05 \pm 0.05$. More importantly, this means that the net electron drift speed is only $v_{d e}=2-3 v_{t i}$, and since the parallel ion drift is $v_{d i} \approx 2 v_{t i}$, it follows that parallel electron and ion drifts are almost equal, $\left|v_{d e}\right| \approx\left|v_{d i}\right|$. Nevertheless, the two species drift in opposite directions, $\boldsymbol{v}_{d e} \boldsymbol{\bullet}_{d i}<0$, with the electrons propagating parallel to the magnetic field direction and the ions propagating anti-parallel to the magnetic field direction.

Wave parallel propagation is parallel to the ion drift but antiparallel to the electron parallel drift, $\boldsymbol{k}_{z} \boldsymbol{\bullet}_{\boldsymbol{d}}<0$, another indication that the mode detected is not a SMIA wave. Also, the mode cannot be explained based on other wave-destabilization mechanisms, such as the two-stream instability ("Buneman") mechanism, where there is a relative drift between ions and electrons. This mechanism requires rather large relative drift and cold populations, whereas in the experiment the relative drift is small (2-3v $\left.v_{t i}\right)$ and the temperatures are large, $T_{i||}=0.19 \mathrm{eV}, T_{i \perp}=0.22 \mathrm{eV}$ and $T_{e}=0.21 \mathrm{eV}$. The wave frequency predicted by the two-stream instability would be $\omega_{r}=\omega_{\mathrm{pe}}\left(\mathrm{m}_{\mathrm{e}} / \mathrm{m}_{\mathrm{i}}\right)^{1 / 3} \approx 5.6 \times 10^{4} \times\left(5 \times 10^{9}\right)^{1 / 2} \mathrm{rad} / \mathrm{s} \times(251,736)^{-1 / 3} \approx 90 \mathrm{Mrad} / \mathrm{s}$, incommensurate with the observed frequency.

\section{Regimes}

A distinct classification of SMIA and D'Angelo instabilities can be made based on the sign of $\sigma^{2}=1-\left(u \omega_{c i}\right)^{-1} d v_{d i} / d x$, as indicated by Gavrishchaka et al. [1999]: positive values correspond to SMIA waves and negative ones to the D'Angelo instability. The $\sigma$ sign depends on the combination of the shear $d v_{\mathrm{di}} / d x$ and the sign of the wavevector ratio $k_{z} / k_{y}$. Fig. 75 summarizes the two possible regimes. The first and the third quadrant give the parameters range suitable for D'Angelo waves, whereas the second and the fourth one give the parameters for SMIA waves. Experimental data points are represented by circles here. For the open circle, $\sigma^{2}=-1.46 \pm 0.03$, while for the three filled circles, $\sigma^{2}=1.35 \pm 0.03,1.42 \pm 0.03,1.64$ \pm 0.04 , respectively. 


\section{Chapter VI. Discussions and Conclusions}

The initial stage of the work presented in this dissertation consisted of creating sheared parallel ion flow in barium-plasma produced in a $\mathrm{Q}$ machine. Barium was chosen because it is one of the alkali or alkali-earth metals suitable for producing plasma in a $\mathrm{Q}$ machine and because its atomic-energy levels have a structure that is suitable for laser-induced fluorescence using commercially available lasers and optical elements.

Two methods for creating shear were designed and used. In the first method, the Q machine was set-up in the single-ended configuration with ions and electrons being created at one end of the device. The plasma-column boundary was a cold termination plate, except for a centimeter-scale-diameter channel along the cylindrical axis that terminated on a positivelybiased electrode $15 \mathrm{~cm}$ in front of the termination plate. The electrode was either a buttonelectrode or an annular-electrode. Similar results were obtained using either of the two electrodes. The two plasma species were each found to have single, drifting-Maxwellian velocity distributions. The ion distribution was completely measured using the laser-induced fluorescence technique, a direct, noninvasive method allowing the determination of the ion drift velocity and ion temperature. The measurements were performed both in the parallel and in the perpendicular directions at an axial distance $z=46 \mathrm{~cm}$ from the north ionizer and across the entire plasma column. By scanning across the plasma column, the radial profile of ion drift velocity and ion temperature (both parallel and perpendicular) are found. A small iontemperature anisotropy was detected $T_{i \perp} / T_{i / /} \sim 1.17$, but there were no structures in the temperatures' radial profiles.

Shear was induced by properly adjusting the potentials applied to the electrode and to the termination plate, respectively. The positive potential of the electrode reduced the parallel drift velocity of the ions located at radii smaller than the electrode radius. The negative potential of the termination plate increased the drift velocity of the ions drifting at radii larger than the electrode radius. The induced inhomogeneity in the parallel-ion-drift velocity led to a cylindrically-symmetric parallel-velocity shear. The position of the shear region was correlated with the edge of the electrode. The shear magnitude increased with increasing potential difference between the electrode and the termination plate in the range of 
$0.05 \omega_{c i}<d v_{d i} / d x<0.22 \omega_{c i}$ if the potential difference exceeded $28 \mathrm{~V}$. Negligible shear was created if the potential difference was less than $28 \mathrm{~V}$ and no increase in the shear was achieved even for applied potential differences larger than $50 \mathrm{~V}$. The shear in the perpendicular ion drift velocity was negligible (less than $0.01 \omega_{c i}$ ). Over the shear region, the change in the ion drift velocity was $\Delta v_{d i} \sim 200 \mathrm{~m} / \mathrm{s}$, representing approximately $0.45 v_{t i z}$. The shear region extended over an annular dimension $L=1.4 \mathrm{~cm}$ representing $4.5 \rho_{\mathrm{i}}$.

Electron drift velocity had a homogeneous radial profile across the entire plasma column and, unlike the reports from previous experiments [D'Angelo and Motley, 1962; Sato et al., 1977], no electron-drift channel associated with the radial position of the electrode was detected. The absence of electron velocity shear means that the parallel ion shear in the electron frame $\left(d v_{d i} / d x\right)_{\mathrm{e}}$, the electron shear in the ion frame $\left(d v_{d e} / d x\right)_{\mathrm{i}}$, and the ion shear in the laboratory frame $d v_{d i} / d x$ have identical absolute values.

The combination of parallel-ion-velocity shear and relative electron drift destabilized multi-harmonic ion cyclotron waves. The theoretical model of Gavrishchaka et al. [2000] and Ganguli et al. [2002] was used for comparison with the experimental results. Key elements required by the model were fulfilled in the experiments: shear was created in the parallel-ionvelocity drift of a single drifting ion population; shear in the electron and lab frame were equal; no density gradients existed in the plasma; no radial electric field was created by inserting a biased electrode in the plasma, thus the ion $\boldsymbol{E} \times \boldsymbol{B}$ drift was negligible; the long wavelength limit $k \lambda_{D}<1$ was accomplished. Moreover, the local approximation limit of the model was valid since the shear layer thickness was several times larger than the ion gyroradius. Slab geometry was valid since the shear-layer thickness was smaller than the shear-layer radius.

Three distinct regimes were documented: (a) the inverse ion cyclotron damping regime, (b) the reduced ion cyclotron damping regime and (c) the enhanced ion cyclotron damping regime. In (a), shear inverted the usual sign of the ion cyclotron damping term, thus providing wave growth. The small relative electron drift measured $\left(v_{d e}=30-60 v_{t i}\right)$ could not destabilize the detected ion cyclotron waves since the electron drift was not larger than the wave parallel phase velocity. In homogeneous plasmas, electron drift must exceed the wave phase velocity for electron-Landau-damping of the wave to be inverted and the electron drift must increase further to destabilize higher harmonics. 
In the reduced ion cyclotron damping regime, waves were destabilized by inverse electron Landau damping. The reduction in the ion cyclotron damping made possible the destabilization of higher harmonics by a relatively small electron drift $\left(v_{d e}=70-100 v_{t i}\right)$. The highest harmonics had frequencies in the lower-hybrid frequency range.

By switching the magnetic field direction, the sign of parallel-velocity shear changed. Thus, ion cyclotron damping was enhanced, a case in which not even a larger electron drift $\left(v_{d e}=150 v_{t i}\right)$ could destabilize the waves. Using a homogeneous plasma as a benchmark, the measured critical electron drift was $\left(v_{d e}\right)_{\text {crit }}=120 v_{t i}$, consistent with the theoretical predictions [Drummond and Rosenbluth, 1962; Kindel and Kennel, 1971].

The experiments characterized the multi-harmonic ion cyclotron wave properties. The number of wave harmonics increased with shear magnitude. For a constant shear, the number of wave harmonics increased with decreasing wave perpendicular wavelength, $k_{\perp} \rho_{i} \sim 1$, consistent with the model. For short parallel wavelengths, the effect of shear was greatly reduced, as predicted by theory, and no waves were destabilized when the electrode was placed at $25 \mathrm{~cm}$ from the hot plate. The wave azimuthal propagation characteristic was given by $\exp (i 3 \theta)$. This is the first work to document in a Q-machine plasma containing parallelvelocity shear an azimuthal propagation for electrostatic ion cyclotron waves and their harmonics $(n=1,2,3, \ldots)$ different from $\exp (0)$. The theoretical model benchmarked here predicts that shear effects do not appear for the case $\exp (0)$ azimuthal propagation.

Ordinarily, the wave frequency appeared slightly below the ion cyclotron frequency, $\omega=0.93 \omega_{c i}$. In all of those cases, the shear was positive and the perpendicular wavevector $k_{y}$ was negative. Both the parallel wavevector components and the parallel electron drift were always positive. In some cases, waves at $\omega=1.06 \omega_{c i}$ were observed. In all of these cases, the shear was positive and $k_{y}>0$, consistent with the ion cyclotron damping factor maintaining the same sign as the $k_{y}<0$ case. The wave harmonics were spaced by intervals equal to the ion cyclotron frequency, an indication of the linear nature of the destabilization mechanism.

Measuring the full radial profile of the wave amplitude posed was compromised due to the strong mode sensitivity to probe-induced perturbations within the shear layer. The measured wave amplitude increased within the shear layer. The spatial-averaged signal collected by the electrode channel-termination yielded the largest wave amplitude. The nonintrusive method of determining the plasma density fluctuations $\delta n / n$, based on the LIF- 
measured, spatially resolved, perturbed ion velocity distribution $f_{1}(t, r, v)$, could not be used due to the small amplitude of the wave. This method requires the collection optics signal output to be phase-locked with the wave. Because the magnitude of the perturbed ion velocity distribution depends on both the mode amplitude and the ion metastable state density, when ever any of these two quantities is small, the measurement of $f_{1}$ is difficult to perform. Attempts to perform measurements of $f_{1}$ failed due to these reasons. In these experiments, the ion metastable density is low in the inner half of the channel, i.e. the region whose radius equals the inner radius of the annular electrode (or approximately half the radius of the button electrode). This region between the two shear layers corresponds to a rather flat ion drift velocity radial profile where the waves are less likely to exist. More reliable determinations of $f_{1}$ can be only obtained outside this region where the conventional methods also yield reliable results.

The timeseries of the mode has a spiky nature, strongly resembling both space measurements of parallel electric field timeseries of a multi-harmonic ion-cyclotron wave and the computer-simulations of the model.

The second method for creating parallel-ion-velocity shear consisted in setting the Q machine in the double-ended configuration and using no electrodes inserted in the plasma. In this configuration, electrons were created at both ends but ions were created at one end only. Producing ions only at one end was achieved by either trapping the ions in the sheath in front of the ionizer by applying a large negative bias to that ionizer, or by not using the atomic oven located at one of the ends.

Each of the two ionizers was independently biased at negative potentials. The surface of the ionizers was believed to be non-uniformly coated with rhenium and presented numerous visible domains. It is believed that the kinetic effects in the ionizer sheath led to the production of ions streaming away from the ionizer at different radial positions with different parallel velocities such that parallel-ion-velocity shear was created. Another possible explanation for the existence of shear is provided by the independent observation of Skiff and Fasoli [1993] who documented sheared parallel barium-ion flow, using laser-induced fluorescence measurements, in the presence of xenon atoms (atomic mass $131 \mathrm{amu}$ ) at large background pressure. They did not observe sheared flow when introducing a light buffer gas. Therefore, in the experiments presented in this dissertation, the increased atomic barium 
(atomic mass $138 \mathrm{amu}$ ) pressure in the sheath could have caused the parallel ion flow to become sheared. An investigation of this possibility is under way.

The presence of shear was correlated with the presence of ion-temperature anisotropy, $1.2<T_{i \perp} / T_{i z}<2.3$. Electrostatic-probe current-voltage characteristics show that the electron temperature was approximately equal to the perpendicular ion temperature. The negative biases applied to the ionizers led to the thermal insulation of the electron-rich plasma from the ionizers and the electrons and ions were heated such that $T_{e} \geq T_{i \perp}>T_{\text {ionizer }}$. This effect has been previously cited in the literature [Motley, 1975], where it is explained that the parallel ion temperature reduces and the perpendicular component increases due to the electrostatic ion acceleration in the sheath.

Laser-induced fluorescence measurements showed that the perpendicular ion drift velocity was homogeneous across the plasma column but the parallel ion drift velocity had a shear region located at about $0.4 \mathrm{~cm}$ from the magnetic field axis. In normalized terms, shear could be created in the range of $0.1<\left|\omega_{c i}{ }^{-1} d v_{d i} / d x\right|<0.65$ by controlling the ionizers' bias voltages. The shear-layer position was at $x=0.4 \mathrm{~cm}$ in some experiments and up to $x=0.9 \mathrm{~cm}$ in others. The shear region was 1.5 to 3.2 times larger than the ion gyroradius. The measurements of parallel-velocity shear were performed along the $x$-axis at $y=0$. To obtain more insight on the profile of the ion drift velocity, a two-dimensional scan, both along the $x$ axis and along the $y$-axis, was performed [E. Reynolds, private communication]. This showed the non-cylindrical symmetry of the two-dimensional structure of the shear region. Nevertheless, the mode was observed to be cylindrically symmetric.

Single-sided probe measurements of the parallel electron drift velocity showed that the radial profile of the parallel electron drift velocity was homogeneous across the plasma column. As in the case of the first method of creating shear in the parallel ion velocity, the absence of electron velocity shear meant that the parallel ion shear in the electron frame $\left(d v_{d i} / d x\right)_{\mathrm{e}}$, the electron shear in the ion frame $\left(d v_{d e} / d x\right)_{\mathrm{i}}$, and the ion shear in the laboratory frame $d v_{d i} / d x$ had identical values.

The spontaneously growing ion acoustic waves detected in these experimental conditions were experimentally characterized and compared with the isotropic-plasma model of SMIA waves proposed by Gavrishchaka et al. [1998, 1999]. This model is the lowfrequency limit of the shear-modified ion-cyclotron waves model mentioned before. Key elements required by the model were fulfilled in the experiments: shear was created in the 
parallel-ion-velocity drift of a single drifting ion population; shear in the electron and lab frame were equal; no density gradients existed in the plasma; no radial electric field was created. Thus the ion $\boldsymbol{E} \times \boldsymbol{B}$ drift was negligible; the long wavelength limit $k \lambda_{D}<1$ was accomplished. Also, the local approximation used by theory could be applied in the majority of cases since the shear layer thickness was larger than the ion gyrodiameter. For some of the experimental conditions, the shear layer thickness was slightly smaller than the ion gyrodiameter. In those instances, a cylindrical theory, as well as a nonlocal, anisotropic theory is necessary for a more accurate comparison with the experimental data.

The significance of the Doppler shifted wave frequency was emphasized, since without it results can be interpreted erroneously. The shift in the wave parallel phase velocity in the ion frame was experimentally measured by comparing the wave phase velocity and the ion sound velocity. The wave always propagated parallel to the electron drift irrespective of the direction of the ion flow. The reduction in ion Landau damping was quantitatively determined by experimentally determining the slope of the distribution function at the wave phase velocity, thus verifying a key prediction of the model. The reduced parallel electron current necessary to destabilize the wave $\left(v_{d e} \sim 100 v_{t i z}\right)$ was also in agreement with the model. In homogeneous plasma, the critical electron drift [Kindel and Kennel, 1971] would have been $\left(v_{d e}\right)_{\text {crit }} \sim 380 v_{t i z}$.

The isotropic theory predicted negative wave growth-rates for the measured wave and plasma parameters. Specifically, the isotropic model could not account for the small wave propagation angle observed since theory predicted rather perpendicular wave propagation $\left(k_{z} / k_{y} \sim 0.1\right)$, whereas experiments showed that $0.7<k_{z} / k_{y}<1.9$. This discrepancy was removed when a comparison with the anisotropic-plasma model [Spangler et al., 2002] was made [Scime et al., 2002]. It was then proved that the reduction in the wave propagation angle was caused by the temperature anisotropy, $T_{i \perp}>T_{i z}$. Increased anisotropy correlated with decreased propagation angle and increased wave growth rate. This new feature is made possible by the oblique propagation of the wave. The wave's ability to be influenced by the shear and thus to optimize its propagation angle for maximum growth rate would not be possible for waves propagating entirely parallel to the magnetic field. The effect of ion and electron temperature anisotropy in the SMIA waves growth and propagation has been first addressed theoretically by Spangler [2001] and subsequently by Spangler et al. [2002]. One of the main results of Spangler et al. [2002] was the derivation of the anisotropic-plasma dispersion relation for 
SMIA waves and of their growth rate. They used analytic theory to document the trend of increasing growth rate, decreasing propagation angle and increasing wave frequency in the case of increasing ion temperature anisotropy $T_{i y} / T_{i z}>1$. Their work shows that in the case that larger anisotropies are achieved by decreasing ion parallel temperature (while keeping $T_{i y}$ and electron parallel temperature $T_{e z}$ fixed) the increase in wave growth rate and wave frequency, along with the decrease in the propagation angle, are rather small (of the order of $50-70 \%$ ). On the other hand, if increased anisotropy was obtained by increasing $T_{i y}$ and, due to the dispersion relation constraints, $T_{e z}$, the increase in the wave growth rate and frequency, along with the decrease in the wave propagation angle, were much more dramatic (around $500 \%$ for the same increase in ion temperature anisotropy).

The results presented here suggest that perpendicular-propagating ion-acoustic waves tend to favor plasmas with unheated ions and that the propagation angle decreases as transverse ion acceleration progresses. This implies that obliqueness would decrease once some transverse ion acceleration has taken place. As transverse ion acceleration progresses, and the wave propagates more parallel (optimum value of $k_{\mathrm{z}} / k_{\mathrm{y}}$ increases), the degree of shear $\left(d v_{\mathrm{diz}} / d x\right) / \omega_{c i}$ would have to increase to maintain the same value of $k_{\mathrm{y}}\left(d v_{\mathrm{diz}} / d x\right) /\left(k_{\mathrm{z}} \omega_{\mathrm{ci}}\right)$ that directly affects the shear modification factor $\sigma$. The measurement of perpendicular phase velocity $\omega_{\mathrm{lr}} / k_{\mathrm{y}}=c_{s z} \sigma k_{z} / k_{y}$ documents that, as $k_{\mathrm{z}} / k_{\mathrm{y}}$ increases due to increasing anisotropy, the perpendicular phase velocity becomes larger than the "perpendicular" ion-acoustic speed $c_{\text {sy }}\left(=\sqrt{k_{B}\left(T_{e}+2 T_{i y}\right) / m_{i}}\right)$. This upshift in $\omega_{1 r} / k_{y}$ is opposite to the downshift in $\omega_{1 r} / k_{y}$ to smaller than $c_{\text {sy }}$ predicted by the isotropic-plasma model of the SMIA instability when using the model's optimum values of $k_{z} / k_{y}$. The observed upshift is interpreted to be important for destablizing SMIA waves at large $k_{z} / k_{y}$ for moderate $\left(\left|\left(d v_{d i z} / d x\right) / \omega_{c i}\right|<1\right)$ values of shear. Having values of $k_{z} / k_{y}$ significantly below unity puts constraints on the minimum value of $\sigma$ required for sufficiently shifting the perpendicular phase velocity out of the range associated with strong ion Landau damping.

Wave amplitude peaked at the shear layer. The experiments showed that the wave azimuthal propagation was characterized by $\exp (i \theta)$. The fact that parallel-velocity shear with asymmetric profile helped destabilize radially-symmetric waves can be understood in view of the experimental conditions: plasma had cylindrical symmetry and the waves were primarily 
destabilized by the electron current emitted by the circular hot ionizers. Shear only decreased the ion Landau damping and was not the main wave-driver.

The cylindrical geometry of the normal mode was such that the azimuthal wavevector corresponded to a cylindrical region of a radius within the plasma column and equal to or sometimes larger than the radial position of the shear layer. A cause of this extended region could be the apparently wide range of radii associated with the the shear radial profile.

Comparison of the experimental data with the theory in the low-frequency limit is also valid since in the vast majority of cases $b=\left(k_{y} \rho_{i}\right)^{2} \leq 1$, as required by the model. When this parameter is almost equal to or larger than unity, all of the significant higher harmonics terms should be considered. The case when $b=1.6$ and up to $n=3$ harmonics were detected is the subject of future investigations.

The D'Angelo instability, destabilized by parallel-velocity shear, was also documented and compared to the model of Gavrishchaka et al. [1999]. This instability appeared when $\sigma^{2}=-1.46$, in accordance with the theory which predicted that the D'Angelo instability should be destabilized provided that $\sigma^{2}<0$. It is important to mention that this instability was destabilized by shear in the parallel drift velocity of a single, drifting, unidirectionalMaxwellian ion distribution. Previous experiments [D'Angelo and von Goeler, 1966; Willig et al, 1997; Merlino et al, 1997] reported the D'Angelo instability being destabilized at the interface between two oppositely flowing ion populations. The measurements of parallel-iondrift velocity shear reported in some of the previous experiments were performed using ion energy analyzers that involved ion density contributions and provided flux-averaged shear values. Flux-averaged values of drift velocity can be different from the values obtained from direct measurements of velocity using LIF.

The work presented in this dissertation has important implications on the space observations of ion cyclotron and ion acoustic waves. According to the widely accepted interpretation of observations, the destabilization mechanisms of those waves cannot be those already known, such as inverse electron Landau damping or ion beams. Explaining the existence of those waves requires new small-scale analysis and new possible mechanisms. This new, small-scale analysis is responsible for the modern paradigm of particle acceleration and structure in the auroral region.

Ion acoustic or ion-acoustic-like waves have been seen in various regions of space (in the Earth's bow shock, in association with interplanetary shocks) in conditions reported 
unfavorable for the current-driven instability [Gurnett et al, 1979, Kennel et al, 1982, Gurnett, 1985, Onsager et al, 1989, Hess et al, 1998]. Kintner et al. [1996] interpret broadband lowfrequency waves in the cleft ionosphere as current-driven electrostatic waves, perhaps a mixture of ion cyclotron and ion acoustic waves. Wahlund et al. [1994, 1998] reported ionacoustic waves propagating with small $\left(0^{0}\right.$ to $\left.60^{\circ}\right)$ to the magnetic field in the Earth's ionosphere where $T_{e} / T_{i} \sim 1$. Explaining these waves with the current-driven electrostatic ionacoustic instability in homogeneous plasma requires $T_{e} / T_{i}$ to be lowered to an unrealistic value of 0.33 .

The presence of ion acoustic waves in the upward current region of the ionosphere was attributed by Andre et al. [1987] to the electron-drift or the ion-beam-drift destabilization mechanisms. In the same region, both ion acoustic and ion cyclotron waves were [Cattell et al.,1991], also thought to be produced by electron drift or ion beams. Gray et al. [1991] studied the decay of the ion-beam-driven ion acoustic waves in ion holes.

Ion heating due to ion acoustic waves in space has been studied by Vaivads et al. [1998]. They proposed that ion acoustic waves could be excited in the upward current region of the ionosphere by ion beams accelerated by local dc electric fields formed by double layers present in the auroral acceleration region [Vaivads et al., 1998]. They also proposed a model through which ion acoustic waves heat perpendicularly the ion beams in the presence of a cold ion and electron background. Ion heating would be most efficient if the parallel phase velocity of the waves was much larger than the ion perpendicular thermal speed.

Having mostly ion acoustic waves would address the observational fact that no ion cyclotron harmonics of any ion have been seen in the wave spectrum by Kintner et al. [2000]. They suspected that this mixture depends sensitively on $T_{e} / T_{i}$ because in a homogeneous plasma the excitation-threshold value of electron drift speed is lower for ion cyclotron waves that for ion acoustic waves for $T_{e} / T_{i} \sim 1$.

Space observations of streaming ion population and multi-harmonic electrostatic ion cyclotron (EIC) waves were reported by Kintner et al. [1978, 1979] based on the S3-3 satellite [Mozer et al., 1977]. Based on the same satellite observations, Kinter [1980] distinguishes between electrostatic ion cyclotron waves, characterized by frequencies slightly above the ion gyrofrequency and large phase coherence, and harmonic ion cyclotron waves, appearing at frequencies slightly below integer multiples of the ion gyrofrequency and presenting weak phase coherence. 
Wave spectrum analysis from the FAST satellite has shown multi-harmonic ion cyclotron waves in the upper $\mathrm{F}$ region of the atmosphere. Explaining these waves with the classic theory of current-driven ion cyclotron waves in homogeneous plasma would require a large electron drift, since the required electron drift for exciting multi-harmonic EIC waves increases with increasing number of harmonics. Such a high electron drift has not been reported by spacecraft; thus the probability of these waves being excited by field-aligned current is very small. Gavrishchaka et al. [2000] and Ganguli et al. [2002], cited in this dissertation, propose a model through which multi-harmonic ion cyclotron waves could be destabilized by the presence of inhomogeneous parallel ion flow, with or without electron drift.

The future plans related to the work presented in this dissertation consist of experiments in which large shear $\left(d v_{d i} / d x \sim \omega_{c i}\right.$ or larger) will be created by segmenting concentrically the hot plate, using it exclusively for producing ions and accelerating the ions in an electron-free region between the hot plate and a negatively biased mesh a centimeter or so away [Odaka et al., 2000]. This will allow, according to the model, destabilization of ion acoustic waves solely by parallel-velocity shear, which is the large-shear regime of shearmodified ion acoustic waves not studied experimentally yet. Other plans consist of creating shear both in the parallel and perpendicular components of the ion drift velocity, the case when the combination of the two destabilization-mechanisms will be experimentally verified. By using a multi-component plasma, it may be possible to determine experimentally the destabilization threshold of each component.

In conclusion, this dissertation presented two ways of producing shear in the parallel ion velocity in a $\mathrm{Q}$ machine. Shear was measured using laser-induced fluorescence, a direct, noninvasive technique. The effect of shear on electrostatic ion cyclotron and ion acoustic waves, respectively, was benchmarked. Inverse ion-cyclotron damping, induced by parallelion-velocity shear, was experimentally verified for the first time. Destabilization of multiharmonic ion cyclotron waves with frequencies into the lower-hybrid range by the combination of reduced ion-cyclotron damping and inverse electron Landau damping was documented and the comparison with the model was good. Spontaneously-growing ion acoustic waves in the presence of sheared parallel ion flow were detected, characterized and compared favorably with the theoretical model. The effect of ion-temperature anisotropy in the propagation and growth of ion acoustic waves was experimentally verified for the first 
time. The results of the work presented in this dissertation have direct and profound implications on explaining the nature of the destabilization mechanisms and the characteristics of the ion cyclotron and ion acoustic waves observed in space. This work provides detailed experimental evidence that the new destabilization mechanisms for those waves proposed by theory are valid. Together with the theory, this work establishes parallel-velocity shear as a significant destabilizing mechanism for electrostatic ion cyclotron and ion acoustic waves, respectively. 


\section{References}

Agrimson, E., N. D’Angelo and R. L. Merlino, Excitation of ion-acoustic-like waves by subcritical currents in a plasma having equal electron and ion temperatures, Phys. Rev. Lett., 86, 5285, 2001; Effect of parallel velocity shear on the excitation of electrostatic ion cyclotron waves, Phys. Lett. A, 293, 260, 2002.

Amatucci, W. E., M. E. Koepke, T. E. Sheridan, M. Alport and J. J. Carroll, Self-cleaning Langmuir probes, Rev. Sci. Instrum., 64, 1352, 1993.

Amatucci, W.E., Ph.D. dissertation, West Virginia University, 1994.

Amatucci, W. E., D. N. Walker, G. Ganguli, A. Antoniades, D. Duncan, J. H. Bowles, V. Gavrishchaka, M. E. Koepke, Plasma response to strongly sheared flow, Phys. Rev. Lett, 77, 1978, 1996.

Amatucci, W. E., Inhomogeneous plasma flows: A review of in situ observations and laboratory experiments, J. Geophys. Res., 104(A7), 14481, 1999.

Andre, M., H. Koskinen, G. Gustafsson, R. Lundin, Ion waves and upgoing ion beams observed by the Viking satellite, Geophys. Res. Lett., 14, 463, 1987.

Andre, M., P. Norqvist, L. Andersson, L. Eliasson, O. Norberg, A. I. Ericksson. B. Holback, Ion energization mechanisms at $1700 \mathrm{~km}$ in the auroral region, J. Geophys. Res., 103, 4199, 1998.

Bernstein, I. B. and R. M. Kulsrud, Phys. Fluids, 3, 937, 1960.

Brown, I. G., Phys. Fluids, 15, 2254, 1972.

Carroll, J. J., Ph.D. dissertation, Physics Dept., West Virginia University, 1997.

Cattell, C. A., F. S. Mozer, I. Roth, R. R. Anderson, R. C. Elphic, W. Lennartsson, E. Ungstrup, ISEE 1 observations of electrostatic ion cyclotron waves in association with ion beams on auroral field lines from $\sim 2.5$ to $4.5 R_{E}$, J. Geophys. Res., 96, 11421, 1991

Cerkovnik, R., M.S. thesis, West Virginia University, 1989.

Chen, F. F., Introduction to plasma physics and controlled fusion, Plenum Press, New York, 1984.

Correll, D. L., Ph.D. dissertation, University of California, Irvine, 1976.

Correll, D. L., N. Rynn, and H. Boehmer, Phys. Fluids, 18, 1800,1975.

Dakin, D. R., T. Tajima. G. Benford, N. Rynn, J. Plasma Phys., 15, 175, 1976.

D'Angelo, N. and R. W. Motley, Electrostatic oscillations near the ion cyclotron frequency, 
Phys. Fluids, 5, 633, 1962.

D'Angelo, N., The Kelvin-Helmholtz instability in a fully-ionized plasma in a magnetic field, Phys, Fluids, 8, 1748, 1965.

D'Angelo, N. and S. von Goeler, Investigation of the Kelvin-Helmholtz instability in a cesium plasma, Phys. Fluids, 9, 309, 1966.

D’Angelo, N., Ultra-low frequency fluctuations at the polar cusp boundaries, J. Geophys. Res., 78, 1206, 1973.

D'Angelo, N., A. Bahnsen, R. Rosenbauer, Wave and particle measurements at the polar cusp, J. Geophys. Res., 79, 3129, 1974.

Drummond, W.E. and M. N. Rosenbluth, Anomalous diffusion arising from microinstabilities in plasma, Phys. Fluids, 5, 1507, 1962.

Ergun, R. E; Carlson, C. W., McFadden, J. P., Mozer, F. S., Delory, G. T., Peria, W., Chaston, C. C., Temerin, M., Elphic, R., Strangeway, R., Pfaff, R., Cattell, C. A., Klumpar, D., Shelley, E., Peterson, W., Moebius, E., Kistler, L, FAST satellite observations of electric field structures in the auroral zone, Geophys. Res. Lett, 25, $2025,1998$.

Fried, A. D. and R. W. Gould, Longitudinal ion oscillations in a hot plasma, Phys. Fluids, 4, 139, 1961.

Ganguli, G., P. Palmadesso, Y. C. Lee, A new mechanism for excitation of electrostatic ioncyclotron waves and associated perpendicular ion heating, Geophys. Res. Lett., 12, 643, 1985.

Ganguli, G., and P. J. Palmadesso, Electrostatic ion instabilities in the presence of parallel currents and transverse electric fields, Geophys. Res. Lett., 15, 103, 1988.

Ganguli, G., Y. C. Lee, P. J. Palmadesso, Oscillations in a collisionless plasma with parallel currents and transverse dc electric fields, Adv. Space Res., 8, 161, 1989.

Ganguli, G., M. J. Keskinsen, H. Romero, R. Heelis, T. Moore, C. Pollock, Coupling of microprocesses and macroprocesses due to velocity shear: An application to the lowaltitude ionosphere, J. Geophys. Res., 99, 8873, 1994.

Ganguli, G., S. Slinker, V. Gavrishchaka, and W. Scales, Phys. Plasmas, 9, 2321, 2002.

Gavrishchaka, V. V., Ph.D. dissertation, West Virginia University, 1996.

Gavrishchaka, V.V., S. Ganguli, and G. Ganguli, Origin of low-frequency oscillations in the 
ionosphere, Phys. Rev. Lett. 80, 728, 1998; Electrostatic oscillations due to filamentary structures in the magnetic-field-aligned flow: the ion acoustic branch, J. Geophys. Res. 104, 12683, 1999.

Gavrishchaka, V. V., G. I. Ganguli, W. A. Scales, S. P. Slinker, C. C. Chaston, J. P.

McFadden, R. E. Ergun, and C. W. Carlson, Phys. Rev. Lett. 85, 4285, 2000.

Gekelman, W. and R. L. Stenzel, Phys. Fluids, 21, 2014, 1978.

von Goeler, S. and R. W. Motley, Phys. Fluids, 13, 834, 1970.

Gray, P. C., M. K. Hudson, R. Bergman, I. Roth, Simulation study of ion two-stream instability in the auroral acceleration region, Geophys. Res. Lett., 17, 1609, 1991.

Gurnett, D.A., E. Marsch, W. Pilipp, R. Schwen n, H. Rosenbauer, Ion acoustic waves and related plasma observations in the solar wind, J. Geophys. Res., 84, 2029, 1979.

Gurnett, D. A., Plasma waves and instabilities, in Collisionless shocks in the heliosphere: review of current research, Geophys. Monogr. Ser., vol. 85, edited by B. T. Tsurutani and R. G. Stone, p. 207, A. G. U., Washington D. C., 1985.

Hatakeyama, R., N. Sato, H. Sugai, Y. Hatta, Phys. Lett., 63A, 28, 1977; Plasma Phys., 22, $25,1980$.

Heelis, R. A., J. D. Winningham, M. Sugiura, C. Maynard, Particle acceleration parallel and perpendicular to the magnetic field observed by DE 2, J. Geophys. Res., 89, 3893, 1984.

Hendel, H. W. and M. Yamada, Identification of Ion-Cyclotron Drift Instability with Discrete and Continuous Spectra, Phys. Rev. Lett. 33, 1076, 1974.

Hill, D. N., S. Fornaca and M. G. Wickham, Rev. Sci. Instrum. 54, 309, 1983.

Hill, D. N., Ph.D. dissertation, University of California, Irvine, 1983.

Hinnov, E., J. Hirschberg, F. Hoffman, N. Rynn, Phys. Fluids, 6, 1779, 1963.

Hinnov, E., T. Chu, H, Hendel, L. Johnson, Rep. MATT-Q-27, p. 110, Princeton Univ. Plasma Phys. Lab., Princeton, New Jersey, 1970.

Hirschberg, J. G. , J. E. Hesser, E. Hinnov, N. Rynn, Phys. Fluids, 10, 1114, 1967.

Hutchinson, I. H., Principles of Plasma Diagnostics, Cambridge University Press, 1987.

Kennel, C. F., F. L. Scarf, F. V. Coroniti, E. J. Smith, D. A. Gurnett, Nonlocal plasma turbulence associated interplanetary shocks, J. Geophys. Res., 87, 17, 1982.

Kindel, J. M. and C. F. Kennel, Topside current instabilities. J. Geophys. Res., 76, 3055, 1971. 
Kintner, P. M., et al., Geophys. Res. Lett., 5, 139, 1978; J. Geophys. Res., 84, 7201, 1979; Geophys. Res. Lett. 23, 1873, 1996; J. Geophys. Res. 105, 21237, 2000; Phys. Plasmas 7, 2135, 2000.

Kline, J. L., Ph.D. dissertation, West Virginia University, 2002.

Koepke, M. E., W. E. Amatucci, J. J. Carroll, T. E. Sheridan, Experimental verification of the inhomogeneous energy-density driven instability, Phys. Rev. Lett., 72, 3355, 1994.

Koepke, M. E., J. J. Carroll and M. W. Zintl, Excitation and propagation of electrostatic ioncyclotron waves in plasma with structured transverse flow, Phys. Plasmas, 5, 1671, 1998.

Koepke, M. E., J. J. Carroll and M. W. Zintl, Laboratory simulation of broadband ELF waves in the auroral ionosphere, J. Geophys. Res., 104 (A7), 14397, 1999.

Koepke, M. E., M. W. Zintl, E. M. Johnson, E. W. Reynolds and T. N. Good, Counterstreaming ion beams with inhomogeneous density profiles, in Proceedings of the Int'l Conf. Plasma Phys., Quebec, 2000, p. 45.

Koepke, M. E., M. W. Zintl, C. Teodorescu, E. W. Reynolds, G. Wang, and T. N. Good, Inhomogeneous magnetic-field-aligned ion flow measured in a $\mathrm{Q}$ machine, Phys. Plasmas, 9, 3225, 2002.

Loranc, M., W. Hanson, R. A. Heelis, J.-P. Maurice, A morphological study of vertical ionospheric flows in the high-latitude F region, J. Geophys. Res., 96, 3627, 1991.

Merlino, R. L., T. An, J. Willig, N. D'Angelo, The effect of negative ions and neutral particle collisions on the parallel velocity shear instability, in Double Layers: Potential Formation and Related Nonlinear Phenomena in Plasmas, Sendai Plasma Forum, World Scientific ed., Singapore, p. 290, 1997.

McFadden, J. P., C. W. Carlson, R. E. Ergun, F. S. Mozer, M. Temerin, W. Peria, D.

Klumpar, E. Shelley, W. Peterson, E. Moebius, L. Kistler, R. Elphic, R. Strangeway, C. Cattell, R. Pfaff, Spatial structure and gradients of ion beams observed by FAST, Geophys. Res. Lett., 25, 2021, 1998.

Mikhailovskii, A.B., Theory of plasma instabilities, vol. 2, Consultants Bureau, 1974;

Electromagnetic instabilities in inhomogeneous plasma, Institute of physics publishing, 1992.

Motley, R. W., Q machines, Academic Press, 1975.

Odaka, Y., E. Tada, T. Kaneko, R. Hatakeyama, N. Sato, Control of radial profile of field- 
aligned plasma flow velocities, in Proceedings of the Intl. Conference of Plasma Physics, Quebec, Canada, 2000.

Onsager, G., R. H. Holtzworth, H. C. Koons, O. H. Bauer, D. A. Gurnett, R. R. Anderson, H.

Luhr, C., W. Carlson, High-frequency electrostatic waves near Earth's bow shock, $J$. Geophys. Res., 94, 13397, 1989.

Petviashvili, V. I., Sov. Phys. - Tekh. Phys., 12, 144, 1967.

Potemra, T. A., J. Doering, W. Peterson, C. Bostrom, R. Hoffman, L. Brace, AE-C observations of low-energy particles and ionospheric temperatures in the turbulent polar cusp: Evidence for the Kelvin-Helmholtz instability, J. Geophys. Res., 83, 3877, 1978.

Priestley, M. B., Spectral analysis and time series, Academic Press, 1989.

Rynn, N., D. R. Dankin, D. L. Correll, G. Benford, Ion heating by the current-driven electrostatic ion-cyclotron instability, Phys. Rev. Lett., 33, 765, 1974.

Sarfaty, M., S. De Souza Machado, and F. Skiff, Direct determination of ion wave fields in a hot magnetized and weakly collisional plasma, Phys. Plasmas, 3, 4316, 1996.

Sato, N. and R. Hatakeyama, J. Phys. Soc. Jpn., 54, 1661, 1985.

Scime, E. E., A.M. Keesee, R. S. Spangler, M. E. Koepke, C. Teodorescu and E. W.

Reynolds, Evidence for ion thermal anisotropy effects on shear-modified ion acoustic instabilities, Phys. Plasmas, 9, 4399, 2002.

Sheridan, T. E., and J. Goree, Langmuir-probe characteristic in the presence of drifting electrons, Phys. Rev. E., 50, 2991, 1994.

Skiff, F., and F. Anderegg, Direct observation of plasma dielectric motion, Phys. Rev. Lett., 59, 896, 1987.

Skiff, F. and A. Fasoli, Rapid Cross-Field Ion Transport In A Plasma With Sheared Parallel Flow, Physics Lett. A 184, 104, 1993.

Smith, C. and S. von Goeler, Kelvin-Helmholtz instability for a collisionless plasma model, Phys. Fluids, 11, 2665, 1968.

Spangler, R. S., M.S. thesis, West Virginia University, 2001.

Spangler, R. S., E. E. Scime, G. Ganguli, Parallel inhomogeneous flows in a thermally anisotropic plasma: The electrostatic ion acoustic branch, Phys. Plasmas, 9, 2526, 2002.

Stern, R. A. and J. A. Johnson, Phys. Rev. Lett., 34, 1548, 1975. 
Stern, R. A., D. L. Correll, H. Boehmer, N. Rynn, Nonlocal effects in the electrostatic ion cyclotron instability, Phys. Rev. Lett, 37, 833, 1976.

Stix, T. H., Waves in plasmas, A. I. P. Press, 1996.

Tada, A., T. Kaneko, R. Hatakeyama, N. Sato, Low frequency instabilities arising from radial-profile jump of field-aligned plasma flow velocity, J. Plasma Fusion Res., SERIES 4, 524, 2001.

Teodorescu, C., E. W. Reynolds and M. E. Koepke, Experimental verification of the shearmodified ion acoustic instability, Phys. Rev. Lett., 88, 185003, 2002a.

Teodorescu, C., E. W. Reynolds, M. E. Koepke, Observation of inverse ion cyclotron damping induced by parallel-velocity shear, Phys. Rev. Lett., 89, 105001, 2002b.

Teodorescu, C., M. E. Koepke and E. W., On the role of ion-temperature anisotropy in the growth and propagation of shear-modified ion-acoustic waves, J. Geophys. Res., 108 (A1), 2049, 2003.

Tonks, L. and I. Langmuir, Oscillations in ionized gases, Phys. Rev., 33, 195, 1929.

Vaivads, A., K. Roennmark, T. Oscarsson, M. Andre, Heating of beam ions by ion acoustic waves, Ann. Geophysicae, 16, 403, 1998.

Wahlund, J.-E., P. Louarn, T. Chust, H. de Feraudy, A. Roux, B. Holback, P.-O. Dovner, G. Holmgren, On ion acoustic turbulence and the nonlinear evolution of kinetic Alfven waves in the aurora, Geophys. Res. Lett., 21, 1835, 1994;

Wahlund, J.-E., A. I. Eriksson, B. Holback, M. Boehm, J. Bonnell, P. Kintner, C. Seyler, J. Clemmons, L. Eliasson, D. Knudsen, P. Norqvist, L. Zanetti, Broadband ELF plasma emission during auroral energization, I. Slow ion acoustic waves, J. Geophys. Res., 103, 4343, 1998.

Willig, J., R. L. Merlino, N. D’Angelo, Experimental study of the collisional parallel-velocity shear instability, J. Geophys. Res, 86, 5507, 1997a; Experimental study of the parallelvelocity shear instability, Phys. Lett. A, 236, 223, 1997 b.

Wong, A. Y., N. D’Angelo, R. W. Motley, Phys. Rev. Lett., 9, 415, 1962; A. Y. Wong, R. W. Motley, N. D'Angelo, Landau damping of ion acoustic waves in highly ionized plasmas, Phys. Rev., 133, A436, 1964.

Wu, C. S. and S. Zmuidzinas, Enhanced fluctuations in a magnetized plasma due to a degenerate ion-wave instability, Phys. Rev. Lett., 177, 408, 1969.

Zintl, M. W., J. J. Carroll, M. E. Koepke, C. A. Selcher, W. E. Amatucci and D. N. Walker, 
Ion-cyclotron waves and ion acceleration in the presence of transverse velocity shear, poster no. SM72 B-17, 1996 Fall AGU Meeting, San Francisco, CA. 
Table 1. WVU Q machine barium-plasma parameters

Background pressure

Magnetic field strength

Plasma column length

Plasma column diameter

Plasma density

Plasma potential

Ion/electron mass ratio

Ion cyclotron frequency

Ion plasma/cyclotron

frequency ratio

Ion gyroradius

Ion Debye length

Parallel ion-temperature

Perpendicular ion-temperature

Electron temperature

Parallel ion drift speed:

Perpendicular ion drift speed

Parallel electron drift speed

Ion sound speed

Parallel ion thermal speed

Perpendicular ion thermal speed

Electron thermal speed

Electron gyroradius

Electron Debye length

$$
\begin{aligned}
& \mathrm{P}_{\mathrm{n}}=1.31 \times 10^{-4} \mathrm{~Pa} \\
& \mathrm{~B}=0.05-0.3 \mathrm{~T} \\
& 3 \mathrm{~m}
\end{aligned}
$$

$6.4 \mathrm{~cm}$

$\mathrm{n}=(0.5-5) \times 10^{9} \mathrm{~cm}^{-3}$

$\Phi=-2.5 \mathrm{~V}$ (for grounded ionizers)

$\mu=\mathrm{m}_{\mathrm{i}} / \mathrm{m}_{\mathrm{e}}=2.4 \times 10^{5}$

$\omega_{\text {ci }}(B=0.1 \mathrm{~T})=69.09 \times 10^{3} \mathrm{rad} / \mathrm{s}$

$\omega_{\mathrm{pi}} / \omega_{\mathrm{ci}} \sim 10^{3}$

$\rho_{\mathrm{i}}\left(\mathrm{B}=0.1 \mathrm{~T}, \mathrm{~T}_{\mathrm{iy}}=0.2 \mathrm{eV}\right)=0.5 \mathrm{~cm}$

$\lambda_{\text {Di }}\left(\mathrm{n}=10^{9} \mathrm{~cm}^{-3}\right)=5 \mu \mathrm{m}$

$\mathrm{T}_{\mathrm{iz}}=0.19-0.23 \mathrm{eV}$

$\mathrm{T}_{\mathrm{iy}}=0.23-0.44 \mathrm{eV}$

$\mathrm{T}_{\mathrm{e}}=0.23-0.50 \mathrm{eV}$

$\mathrm{v}_{\mathrm{di}, \mathrm{z}}=0.4-1.1 \mathrm{~km} / \mathrm{s}$

$\mathrm{v}_{\mathrm{di}, \mathrm{y}}=30-250 \mathrm{~m} / \mathrm{s}$

$\mathrm{V}_{\mathrm{de}, \mathrm{z}}=4-80 \mathrm{~km} / \mathrm{s}$

$\mathrm{c}_{\mathrm{s}}=850-930 \mathrm{~m} / \mathrm{s}$

$\mathrm{V}_{\mathrm{ti} \mid}=490-520 \mathrm{~m} / \mathrm{s}$

$\mathrm{v}_{\mathrm{ti \perp}}=490-730 \mathrm{~m} / \mathrm{s}$

$\mathrm{v}_{\mathrm{te}}=220-350 \mathrm{~km} / \mathrm{s}$

$\rho_{\mathrm{e}}\left(\mathrm{B}=0.1 \mathrm{~T}, \mathrm{~T}_{\mathrm{e}}=0.2 \mathrm{eV}\right)=10 \mu \mathrm{m}$

$\lambda_{\text {De }}\left(\mathrm{n}=10^{9} \mathrm{~cm}^{-3}\right)=10 \AA$ 


\begin{tabular}{|c|c|c|c|c|c|c|c|c|c|}
\hline \multicolumn{3}{|c|}{$\mathrm{B}(\mathrm{kG}) \mathrm{P}_{\mathrm{N}}(\mathrm{kW}) \mathrm{P}_{\mathrm{S}}(\mathrm{kW})$} & \multirow{3}{*}{$\begin{array}{r}\begin{array}{r}\text { north } \\
\text { oven }\end{array} \\
\text { off }\end{array}$} & \multirow{2}{*}{$\begin{array}{r}\text { south } \\
\text { oven } \\
\text { on }\end{array}$} & \multirow{2}{*}{$\begin{array}{c}\mathrm{V}_{\mathrm{A}}(\mathrm{V}) \\
35\end{array}$} & \multirow{2}{*}{$\begin{array}{c}\mathrm{V}_{\mathrm{T}}(\mathrm{V}) \\
6\end{array}$} & \multirow{2}{*}{$\begin{array}{c}\begin{array}{l}\mathrm{v}_{\mathrm{di}}(\mathrm{m} / \mathrm{s}) \\
\text { (average) }\end{array} \\
1305\end{array}$} & \multirow{2}{*}{$\begin{array}{c}\mathrm{dv}_{\mathrm{di}} / \mathrm{dx}\left(\omega_{\mathrm{ci}}\right) \\
0\end{array}$} & \multirow{2}{*}{$\begin{array}{c}\text { shear layer } \\
\text { location }(\mathrm{cm})\end{array}$} \\
\hline 2.5 & 0 & 3.1 & & & & & & & \\
\hline & & same & & & & 2 & 1270 & 0.01 & $0.7 \&-1$ \\
\hline & & same & & & & 1 & 1254 & 0.03 & same \\
\hline & & same & & & & 0 & 1235 & 0.05 & same \\
\hline & & same & & & & -1 & 1220 & 0.08 & same \\
\hline & & same & & & & -2 & 1212 & 0.1 & same \\
\hline & & same & & & & -3 & 1189 & 0.12 & same \\
\hline & & same & & & & -4 & 1176 & 0.13 & same \\
\hline & & same & & & & -5 & 1161 & 0.14 & same \\
\hline & & same & & & & -6 & 1150 & 0.16 & same \\
\hline & & same & & & & -8 & 1120 & 0.2 & same \\
\hline 2 & 3.1 & 0 & on & off & 40 & 0 & 586 & 0.13 & $0.7 \&-1.1$ \\
\hline 2 & 2.9 & 0 & on & off & 29 & -3 & 520 & 0.11 & $0.6 \&-1.15$ \\
\hline 2.5 & 3.2 & 0 & on & off & 15 & 0 & 610 & 0 & none \\
\hline 1.4 & 3.2 & 0 & on & off & 15 & -3.7 & 600 & 0 & none \\
\hline
\end{tabular}

Table 2

Device settings used for controlling the parallel ion drift velocity radial profile in the Single Ionizer Single Electrode configuration of the Q machine 


\begin{tabular}{|l|l|l|l|}
\hline$n$ & $d v_{\mathrm{di}} / d x$ & \multicolumn{1}{|c|}{$k_{\mathrm{y}} / k_{\mathrm{z}}$} & \multicolumn{1}{c|}{$\omega_{\mathrm{i}} / n \omega_{\mathrm{ii}}$} \\
\hline 1 & $(0.14 \pm 0.02) \omega_{\mathrm{ii}}$ & $\begin{array}{l}(-2.120 \pm 0.04) \mathrm{cm}^{-1} /(0.024 \pm \\
0.005) \mathrm{cm}^{-1}=-88 \pm 8\end{array}$ & $\begin{array}{l}(156 \pm 5 \mathrm{krad} / \mathrm{s}) /(172.7 \pm \\
3.8 \mathrm{krad} / \mathrm{s})=0.903 \pm 0.006\end{array}$ \\
\hline 2 & $(0.14 \pm 0.02) \omega_{\mathrm{ii}}$ & $\begin{array}{l}(-2.350 \pm 0.02) \mathrm{cm}^{-1} /(0.028 \pm \\
0.005) \mathrm{cm}^{-1}=-84 \pm 8\end{array}$ & $\begin{array}{l}(312 \pm 12 \mathrm{krad} / \mathrm{s}) /(345.4 \pm \\
11 \mathrm{krad} / \mathrm{s})=0.902 \pm 0.006\end{array}$ \\
\hline 3 & $(0.14 \pm 0.02) \omega_{\mathrm{ii}}$ & $\begin{array}{l}(-2.060 \pm 0.03) \mathrm{cm}^{-1} /(0.026 \pm \\
0.005) \mathrm{cm}^{-1}=-79 \pm 7\end{array}$ & $\begin{array}{l}(468 \pm 14 \mathrm{krad} / \mathrm{s}) /(516 \pm 13 \\
\mathrm{krad} / \mathrm{s})=0.906 \pm 0.006\end{array}$ \\
\hline 4 & $(0.14 \pm 0.02) \omega_{\mathrm{ii}}$ & $(-2.070 \pm 0.03) \mathrm{cm}^{-1} /(0.025 \pm$ & $(623 \pm 19 \mathrm{krad} / \mathrm{s}) /(691 \pm 18$ \\
& & $0.005) \mathrm{cm}^{-1}=-83 \pm 8$ & $\mathrm{krad} / \mathrm{s})=0.902 \pm 0.006$ \\
\hline
\end{tabular}

Table 3. Values of the parallel-velocity shear and of the multi-harmonic EIC wave parameters in the case of inverse ion cyclotron damping. Here, $n$ is the harmonic number. 


\begin{tabular}{|cccccccccc|}
\hline $\mathrm{B}(\mathrm{kG})$ & $\mathrm{P}_{\mathrm{N}}(\mathrm{kW})$ & $\mathrm{P}_{\mathrm{S}}(\mathrm{kW})$ & north oven south oven $\mathrm{V}_{\mathrm{n}}(\mathrm{V})$ & $\mathrm{V}_{\mathrm{s}}(\mathrm{V})$ & $\mathrm{V}_{\mathrm{di}}(\mathrm{m} / \mathrm{s})$ & $\left(\mathrm{dv}_{\mathrm{di}} / \mathrm{dx}\right)\left(\omega_{\mathrm{ci}}\right)^{-1}$ & $\begin{array}{l}\text { shear layer } \\
\text { location }(\mathrm{cm})\end{array}$ \\
\hline 1.3 & 3.4 & 2.8 & on & off & 0 & 0 & 460 & 0.21 & +0.9 \\
1 & 3.1 & 2.8 & on & off & 0 & -5 & 470 & -0.1 & +0.6 \\
1.4 & 2.8 & 2.5 & on & on & 0 & -10 & 460 & -0.42 & +0.7 \\
1.3 & 3.4 & 2.8 & on & on & -10 & -30 & 420 & -0.55 & +0.9 \\
1 & 3.1 & 3.1 & on & on & 0 & -25 & 480 & -0.59 & +0.4 \\
1 & 2.7 & 2.6 & on & off & -10 & -30 & 430 & -0.56 & +0.4 \\
1.2 & 2.9 & 2.8 & on & on & 0 & -30 & 440 & -0.63 & +0.5 \\
1.3 & 2.7 & 2.8 & on & on & -30 & 0 & -880 & 0.2 & +0.7 \\
\hline
\end{tabular}

Table 4. Device settings used for controlling the parallel ion drift velocity radial profile in the Double Ionizer No Electrode configuration of the Q machine. Magnetic field direction is southwards. Shear magnitude increases with increasing $\left|V_{N}-V_{S}\right|$. Shear sign switches from positive when $\mathrm{V}_{\mathrm{N}}<\mathrm{V}_{\mathrm{S}}$ to negative when $\mathrm{V}_{\mathrm{N}}>\mathrm{V}_{\mathrm{S}}$, if both atomic ovens are on. 


\begin{tabular}{|c|c|l|l|l|l|l|l|}
\hline North hot plate & South hot plate & North hot & South hot & $\mathrm{Kz}$ & $\mathrm{Ky}$ & Ion drift & Shear \\
\hline power $(\mathrm{kW})$ & power $(\mathrm{kW})$ & plate bias $(\mathrm{V})$ & plate bias $(\mathrm{V})$ & $(1 / \mathrm{cm})$ & $(1 / \mathrm{cm})$ & $(\mathrm{m} / \mathrm{s})$ & $1 /(\mathrm{ms})$ \\
\hline 2.81 & 2.92 & -30 & 0 & 0.27 & 0.13 & 440 & -51.3 \\
2.72 & 2.8 & 0 & -30 & -0.56 & -0.33 & 620 & -45.7 \\
\hline
\end{tabular}

Table 5. Control of the SMIA waves propagation direction adjusting the bias voltages applied to the ionizers.

\begin{tabular}{|c|c|l|l|l|l|l|l|}
\hline North hot & South hot & North hot & South hot & $\mathrm{Kz}$ & $\mathrm{Ky}$ & lon drift & Shear \\
\hline plate power $(\mathrm{kW}$ & plate power $(\mathrm{kW})$ & plate bias $(\mathrm{V})$ & plate bias $(\mathrm{V})$ & $(1 / \mathrm{cm})$ & $(1 / \mathrm{cm})$ & $(\mathrm{m} / \mathrm{s})$ & $1 /(\mathrm{ms})$ \\
\hline 2.7 & 3.3 & -10 & 0 & -0.35 & -0.47 & 450 & -38.1 \\
3.2 & 2.6 & -10 & 0 & 0.29 & 0.52 & 560 & -41.3 \\
\hline
\end{tabular}

Table 6. Control of the SMIA waves propagation direction adjusting the emitted power at the ionizers.

\begin{tabular}{|l|l|l|l|l|l|l|l|l|l|l|}
\hline $\begin{array}{l}\mathrm{T}_{\mathrm{iy}} \\
(\mathrm{eV})\end{array}$ & $\begin{array}{l}\mathrm{T}_{\mathrm{iz}} \\
(\mathrm{eV})\end{array}$ & $\begin{array}{l}\mathrm{T}_{\mathrm{e}} \\
(\mathrm{eV})\end{array}$ & $\begin{array}{l}\omega_{\mathrm{ci}} \\
\left(10^{3}\right. \\
\mathrm{rad} / \mathrm{s})\end{array}$ & $\begin{array}{l}\mathrm{dv}_{\mathrm{di}} / \mathrm{dx} \\
(\mathrm{ms})^{-1}\end{array}$ & $\begin{array}{l}\mathrm{k}_{\mathrm{y}} \\
\left(\mathrm{cm}^{-1}\right)\end{array}$ & $\begin{array}{l}\mathrm{k}_{\mathrm{z}} \\
\left(\mathrm{cm}^{-1}\right)\end{array}$ & $\begin{array}{l}\omega_{\mathrm{r}} \\
\left(10^{3}\right. \\
\mathrm{rad} / \mathrm{s})\end{array}$ & $\begin{array}{l}\omega_{\mathrm{I}} \\
\left(10^{2}\right. \\
\mathrm{rad} / \mathrm{s})\end{array}$ & $\begin{array}{l}\omega\left(10^{3}\right. \\
\mathrm{rad} / \mathrm{s})\end{array}$ & $\begin{array}{l}\mathrm{v}_{\mathrm{di}} \\
(\mathrm{m} / \mathrm{s})\end{array}$ \\
\hline 0.27 & 0.22 & 0.33 & 96.7 & -45 & 0.15 & 0.10 & 9.6 & 2.4 & 14.4 & 480 \\
\hline 0.36 & 0.21 & 0.41 & 96.7 & -54 & 0.12 & 0.20 & 15.2 & 3.1 & 24.4 & 462 \\
\hline 0.46 & 0.19 & 0.51 & 96.7 & -62 & 0.11 & 0.23 & 28.8 & 3.9 & 39.3 & 460 \\
\hline
\end{tabular}

Table 7. Plasma and wave parameters associated with the graphs in Fig. 66 and Fig. 68. 


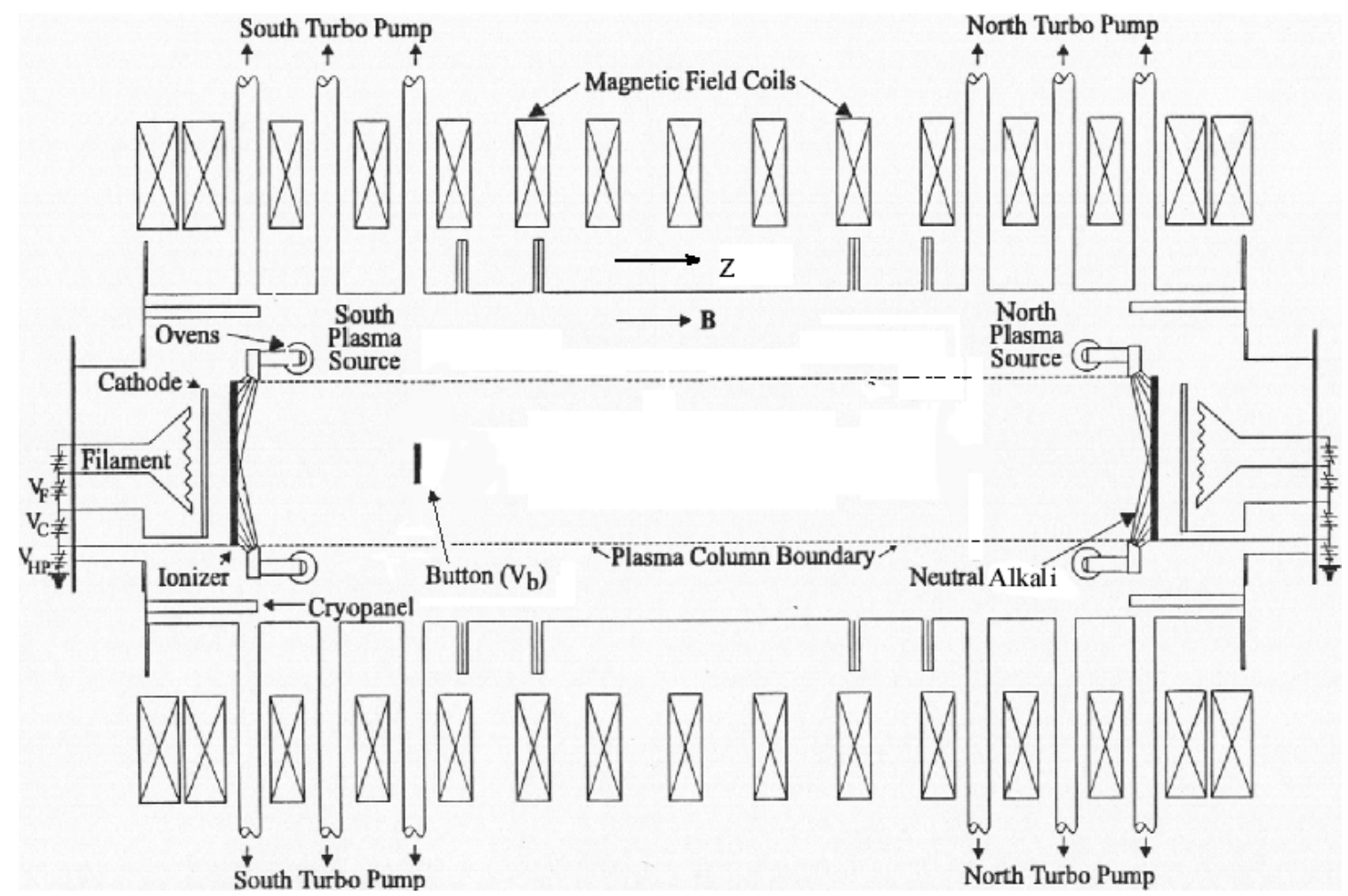

Fig. 1 WVU Q machine (not-to-scale schematic) in the Single Ionizer Single Electrode configuration: here, neither ions nor electrons are produced by the south plasma source. Magnetic field direction can be reversed. $z=0$ at the ionizer from which the magnetic field points out (modified version of a figure by Carroll, [1997]) 


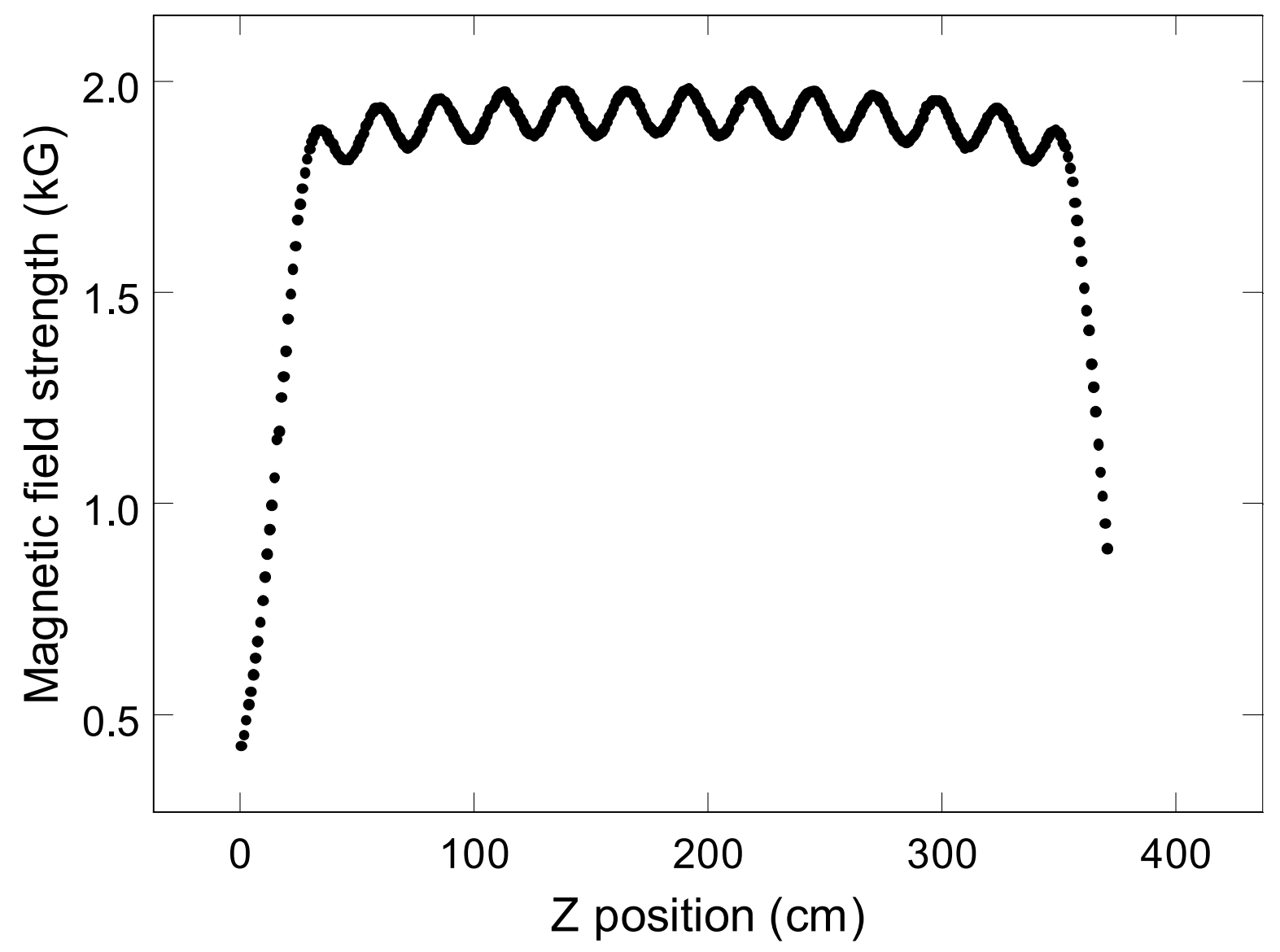

Fig. 2 WVU Q machine magnetic field strength along the Q machine center axis. (reprinted from [Carroll, 1997]). 


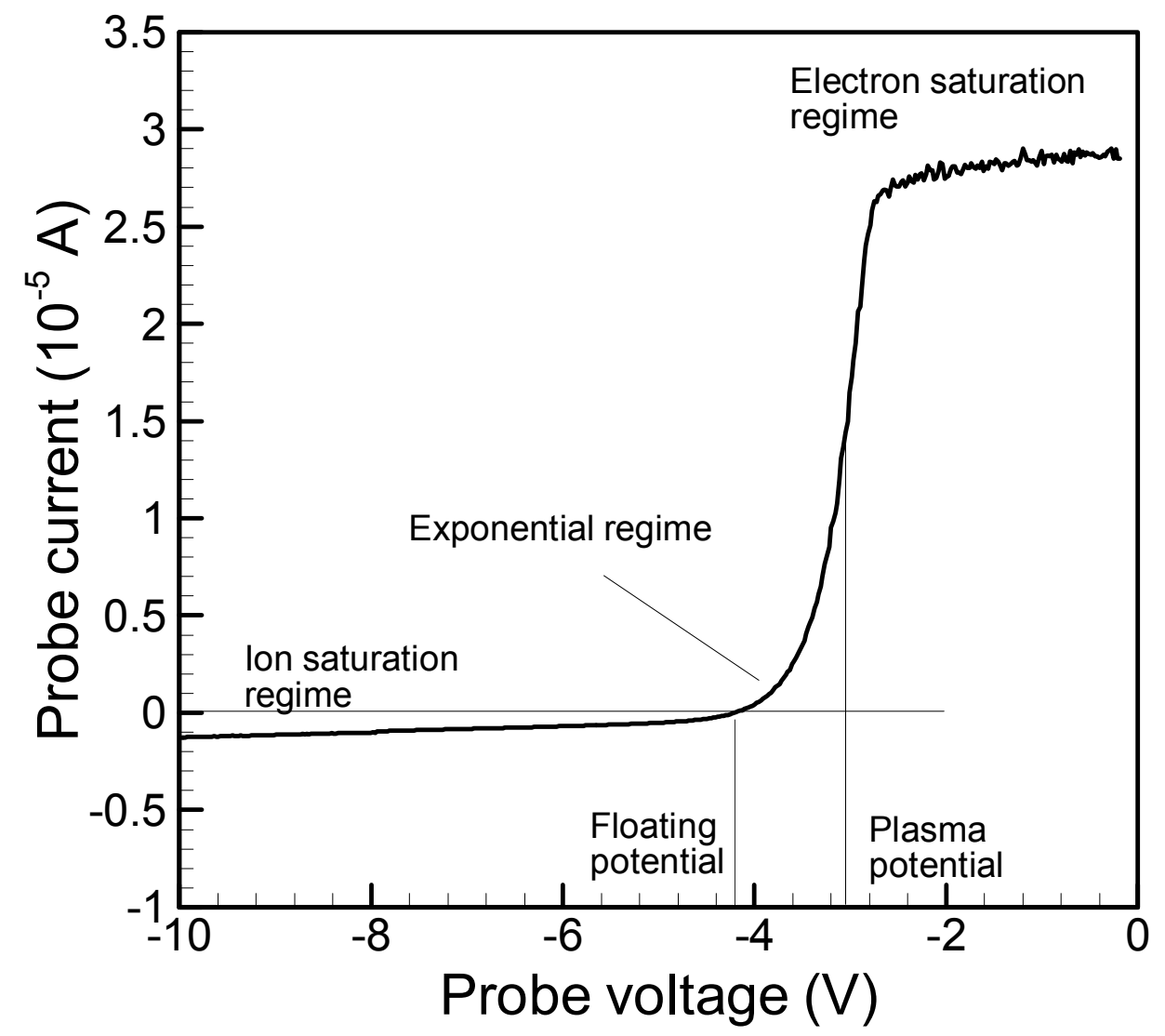

Fig. 3 Typical current-voltage characteristic (measured at $z=69 \mathrm{~cm}$ from the north ionizer) of a Langmuir probe immersed in barium plasma created in the WVU Q machine. Here, $B=0.14 \mathrm{~T}, n=3.2 \times 10^{9} \mathrm{~cm}^{-3}, T_{e}=0.29 \mathrm{eV}$ and $T_{i}=0.23 \mathrm{eV}$. Ionizers are both biased at -1 $\mathrm{V}$. 


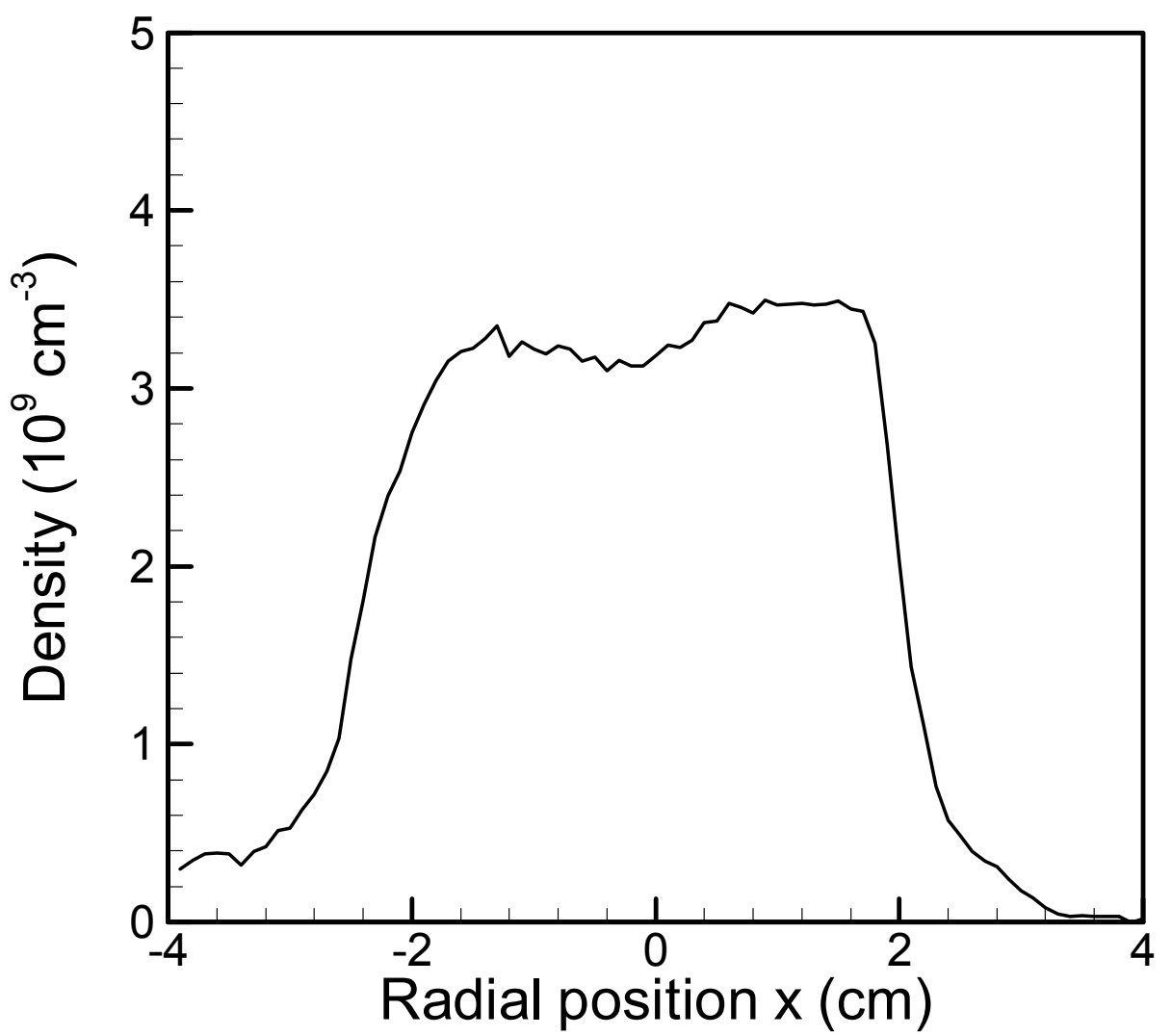

Fig. 4 Barium plasma density radial profile at $z=69 \mathrm{~cm}$ from the north ionizer. $(B=0.14 \mathrm{~T}$ and $V_{\text {probe }}=-7 \mathrm{~V}$ ). Radial position $\mathrm{r}=0$ corresponds to the cylindrical axis of the vacuum chamber. 


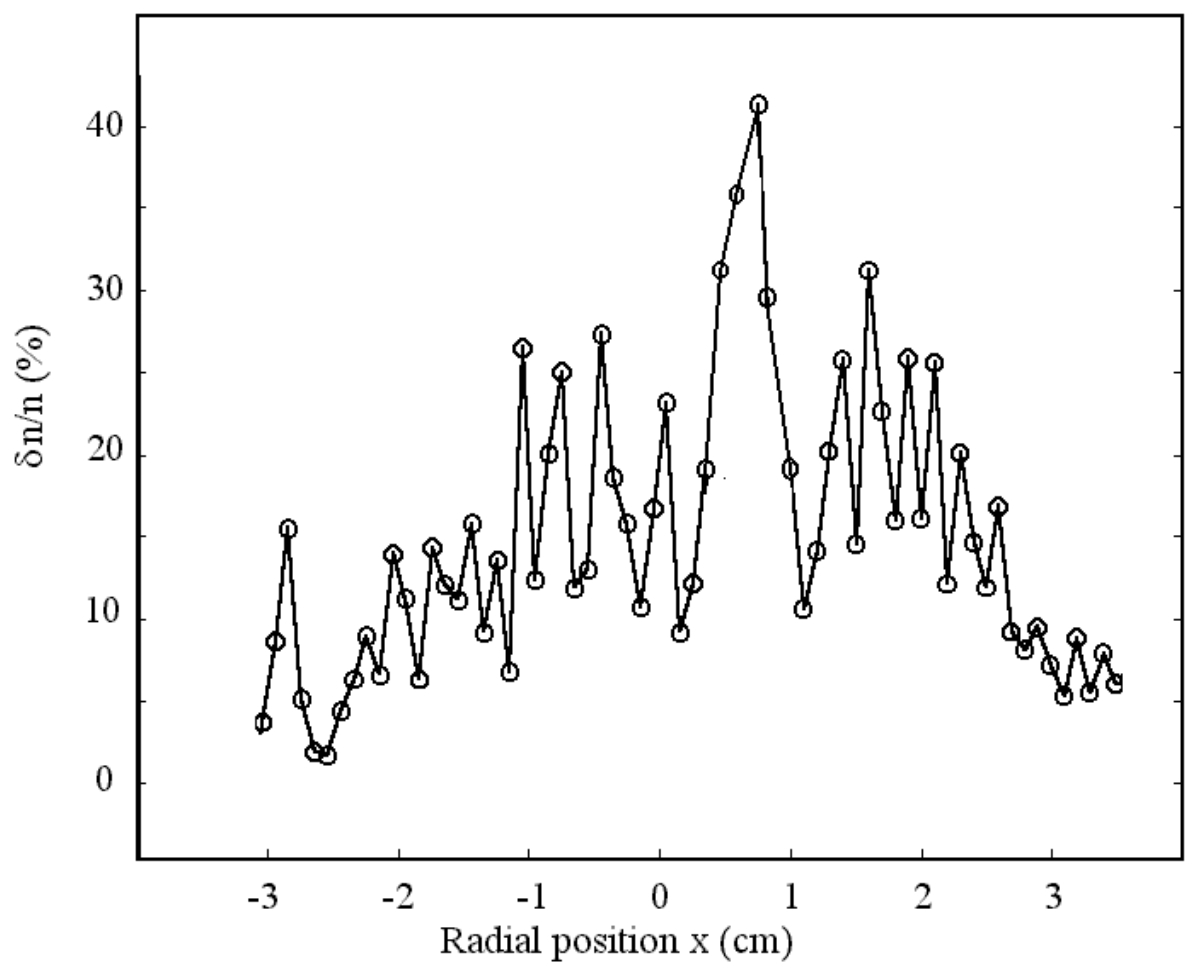

Fig. 5 Barium plasma density fluctuation in the presence of the D'Angelo instability (measured at $z=69 \mathrm{~cm}$ from the north ionizer) when $B=0.15 \mathrm{~T}, V_{\text {probe }}=-5 \mathrm{~V}$ and $n_{0}=$ $2.6 \times 10^{9} \mathrm{~cm}^{-3}$. 

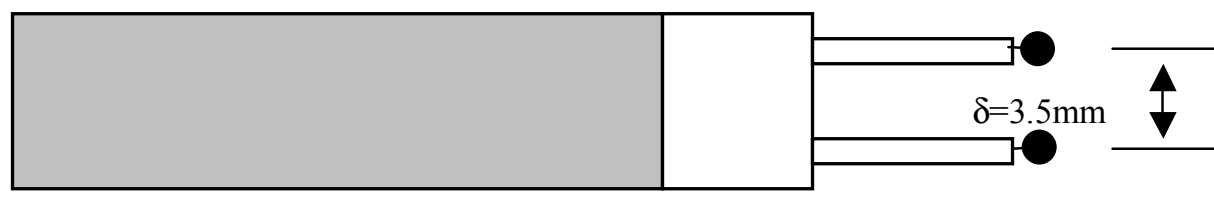

(a)

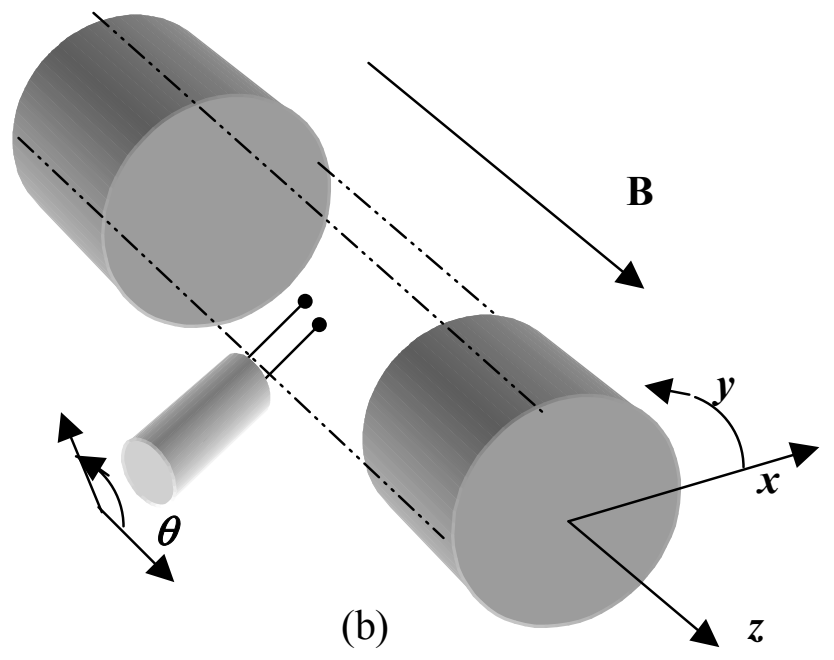

Fig. 6 (a) Two-tip probe array and (b) two-tip probe rotation with respect to the magnetic field lines. 


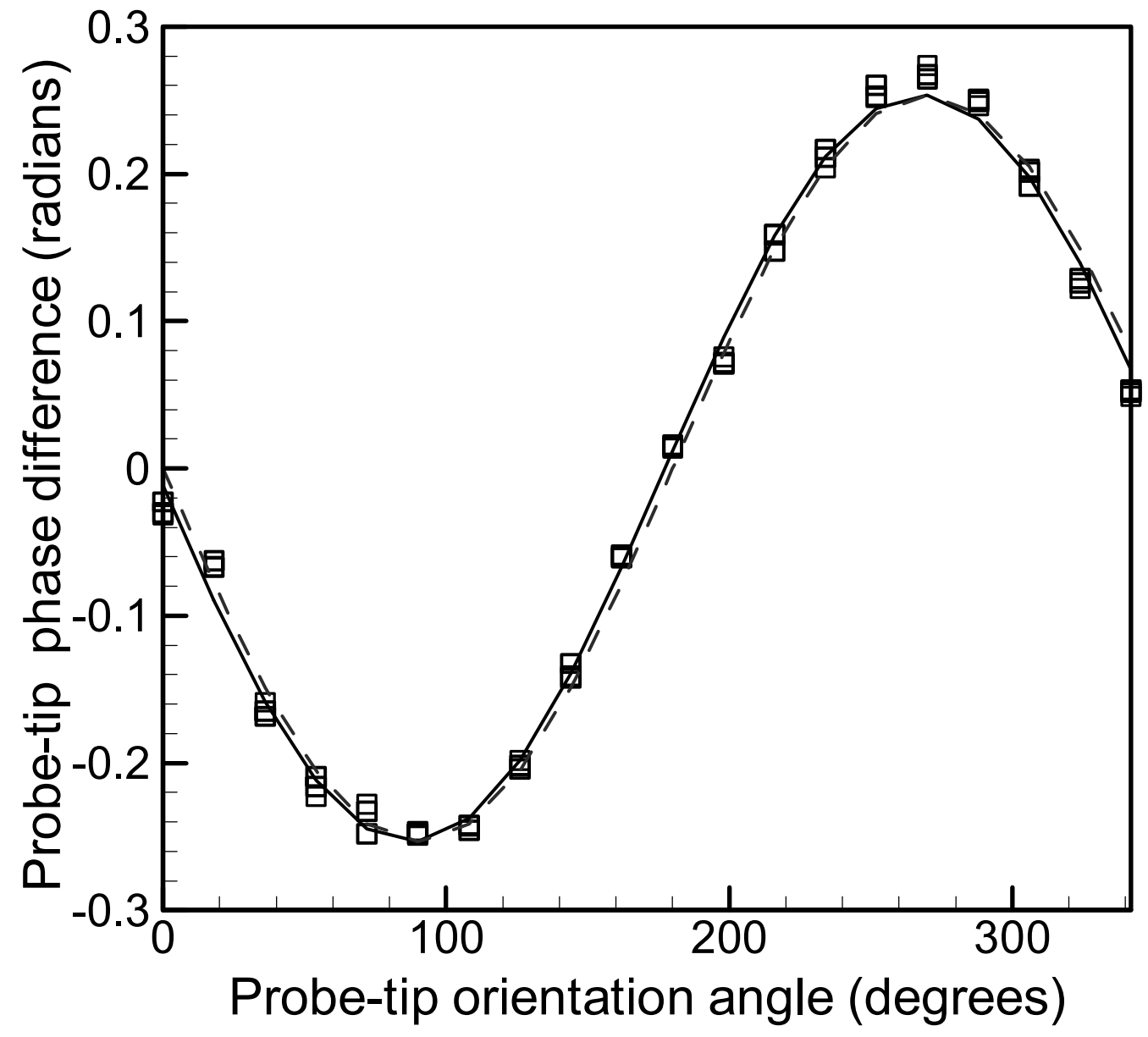

Fig. 7 Dependence of relative phase between probe-array tips on the orientation of the probe array with respect to the magnetic field lines $(B=0.2 \mathrm{~T}$ southward and $z=46 \mathrm{~cm}$ from the north ionizer). The solid line is the best sinusoidal fit, yielding $k_{z}=(-0.038 \pm 0.005)$ $\mathrm{cm}^{-1}$ and $k_{y}=(-3.221 \pm 0.041) \mathrm{cm}^{-1}$; the dashed line is the same sinusoid with $k_{z}=0$. 


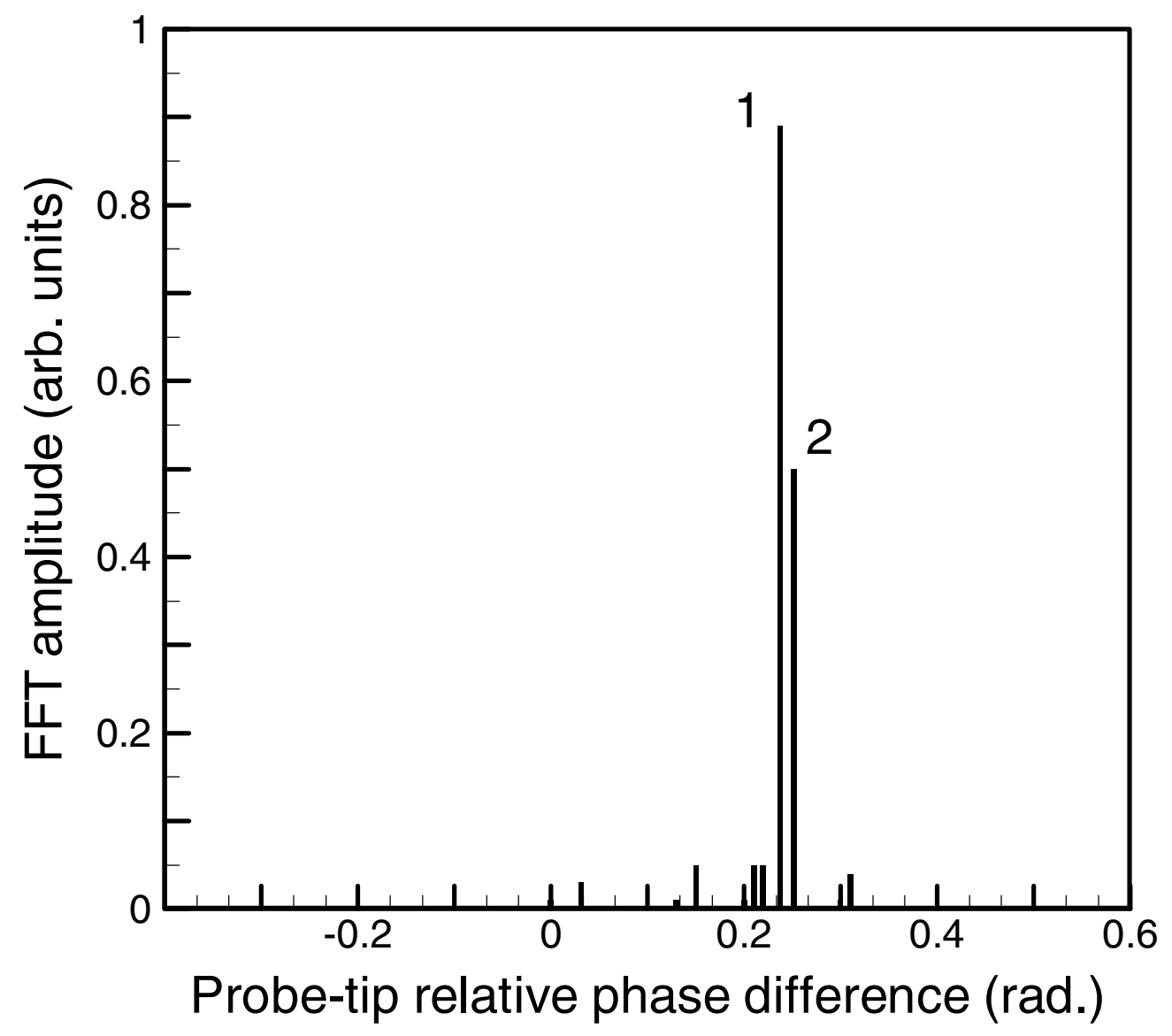

Fig. 8 Perpendicular wavevector component $k_{y}$ spectrum $(B=0.2 \mathrm{~T})$. Probe tip separation is $3.5 \mathrm{~mm}$ yielding $k_{y l}=0.69 \mathrm{~cm}^{-1}$ and $k_{y 2}=0.72 \mathrm{~cm}^{-1}$, respectively, for the two largest components shown. 


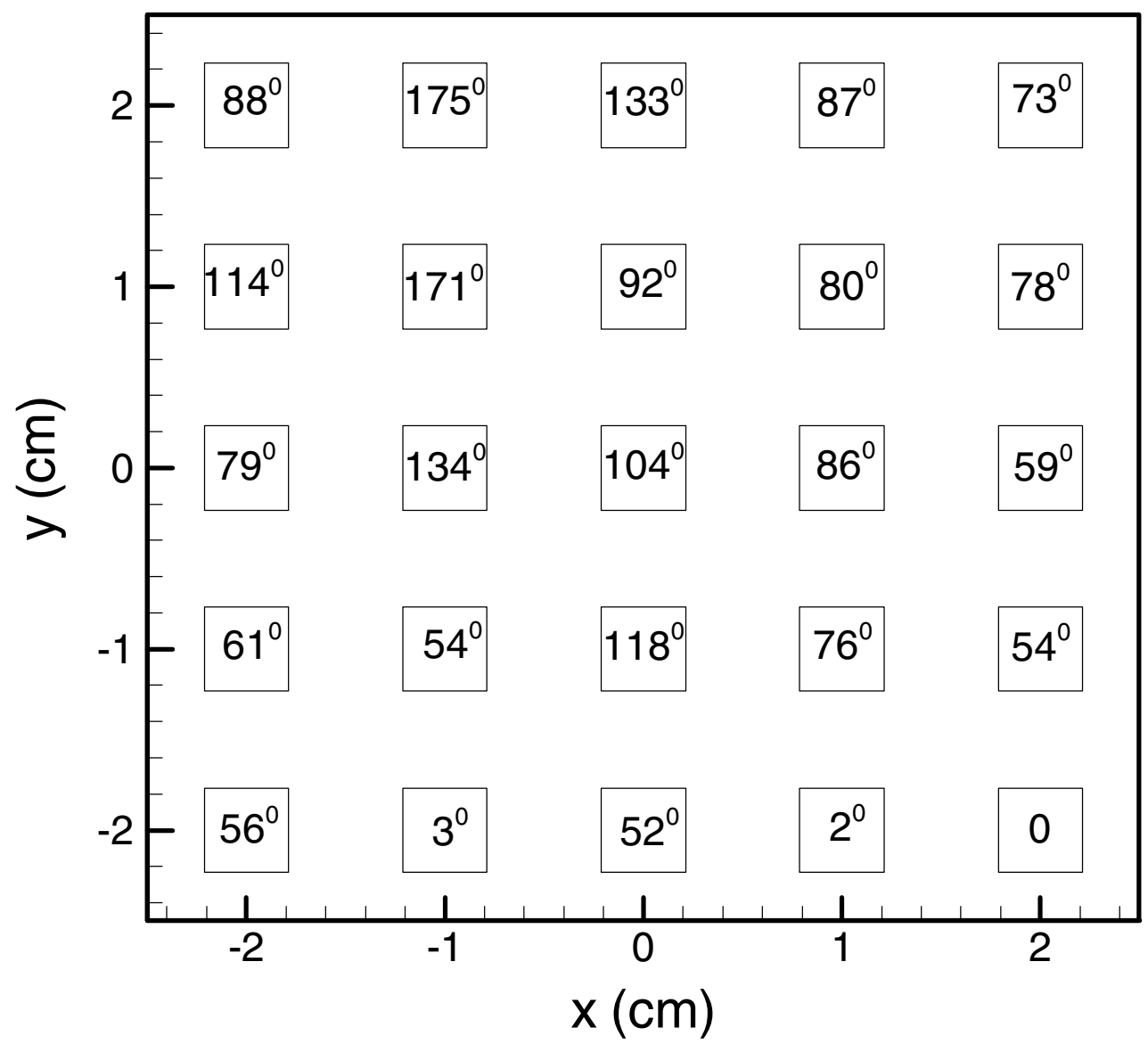

Fig. 9 SMIA wave relative phase between one probe tip at $x=1.5 \mathrm{~cm}, y=1.5 \mathrm{~cm}, z=138 \mathrm{~cm}$ and another probe tip at $-2<x<2 \mathrm{~cm},-2<y<2 \mathrm{~cm}$ and $z=161 \mathrm{~cm}$. The approximately $180^{\circ}$ phase difference between the wave phase measured in the points located at $x=-1 \mathrm{~cm}, y=2 \mathrm{~cm}$ and $x=2 \mathrm{~cm}, y=-2 \mathrm{~cm}$, respectively, indicates an azimuthal mode number $m=1$. 

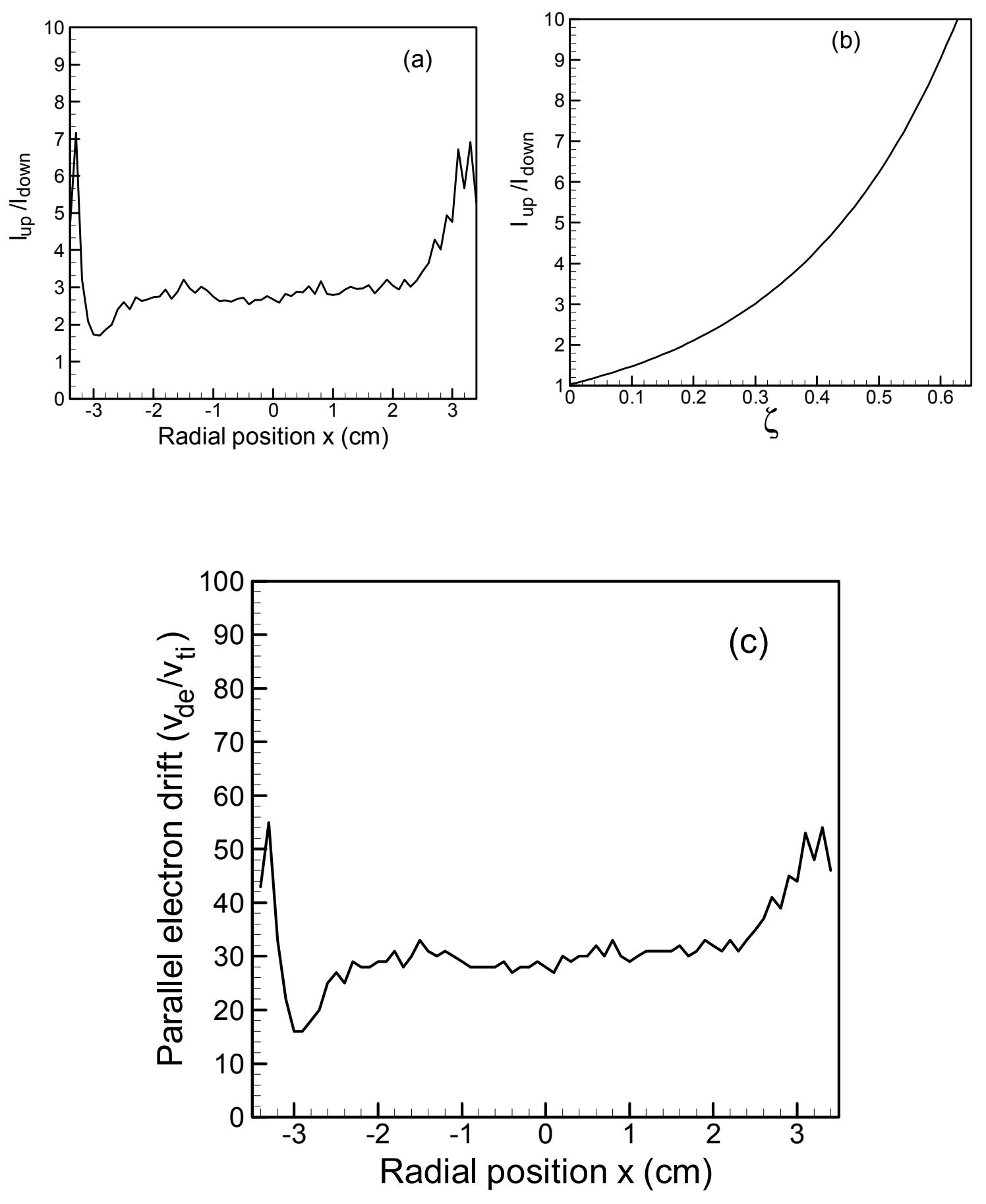

Fig. 10 Parallel electron drift radial profile inferred from single-sided probe measurements $(B=0.15 \mathrm{~T}, z=46 \mathrm{~cm})$. Here, $v_{t i}=520 \mathrm{~m} / \mathrm{s}$. The drift is parallel to the magnetic field. Here, $\zeta=\left|v_{\mathrm{de}} / v_{\mathrm{te}}\right|$. 


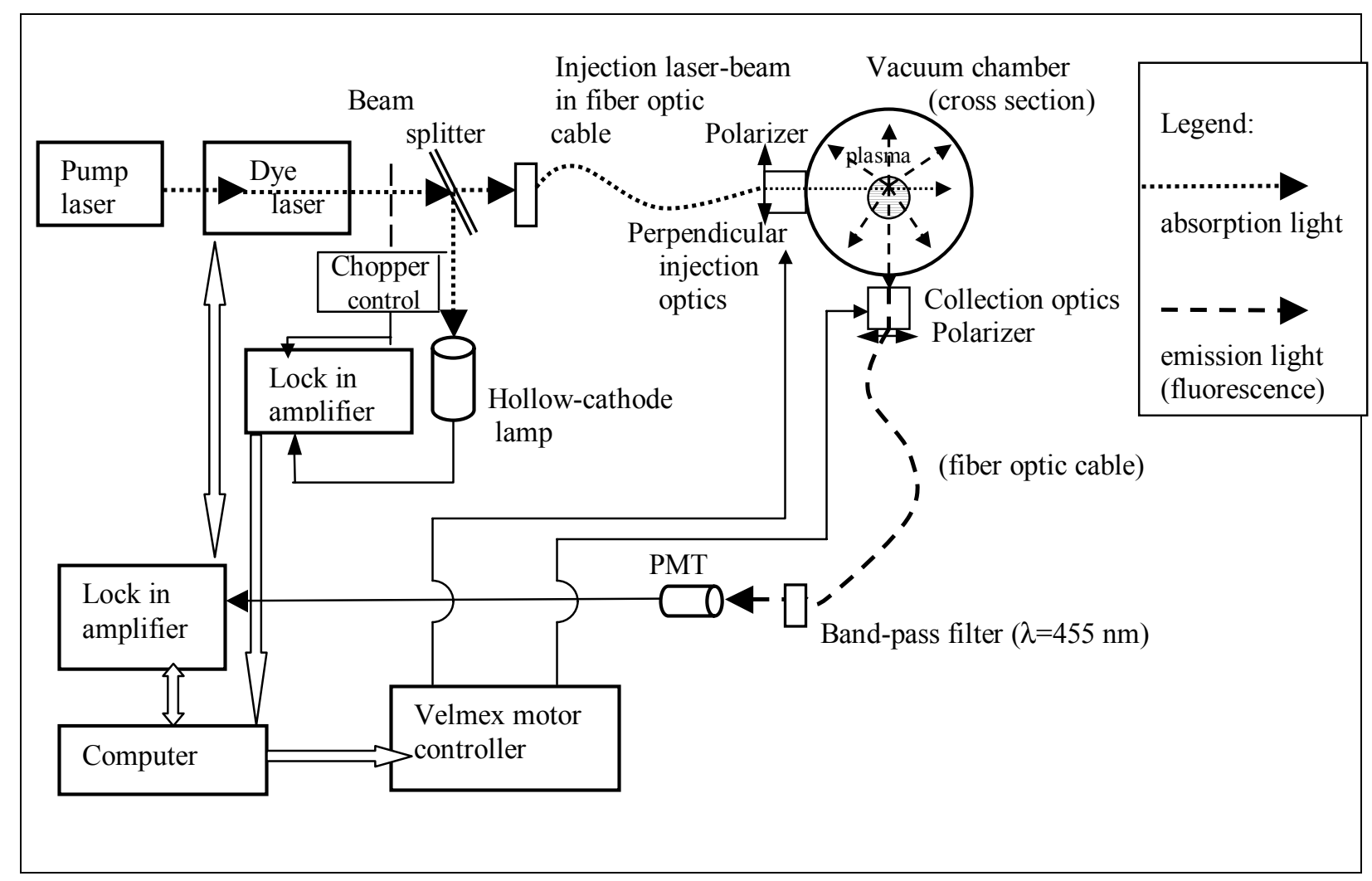

Fig. 11 Schematic diagram of LIF apparatus corresponding to the perpendicularvelocity measurement. 


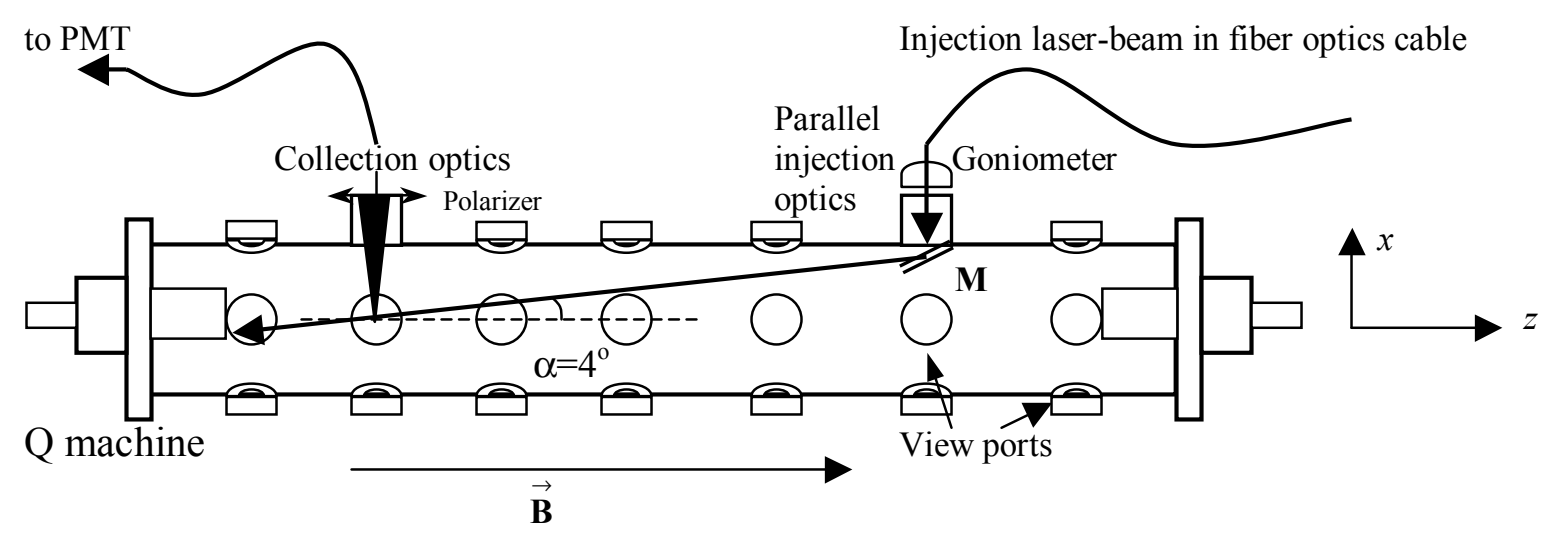

Fig. 12 Schematic of the LIF parallel injection optics mounted on the vacuum chamber (not to scale). Angle $\alpha$ is adjustable by rotating the goniometer that holds the fiber-optic cable. 


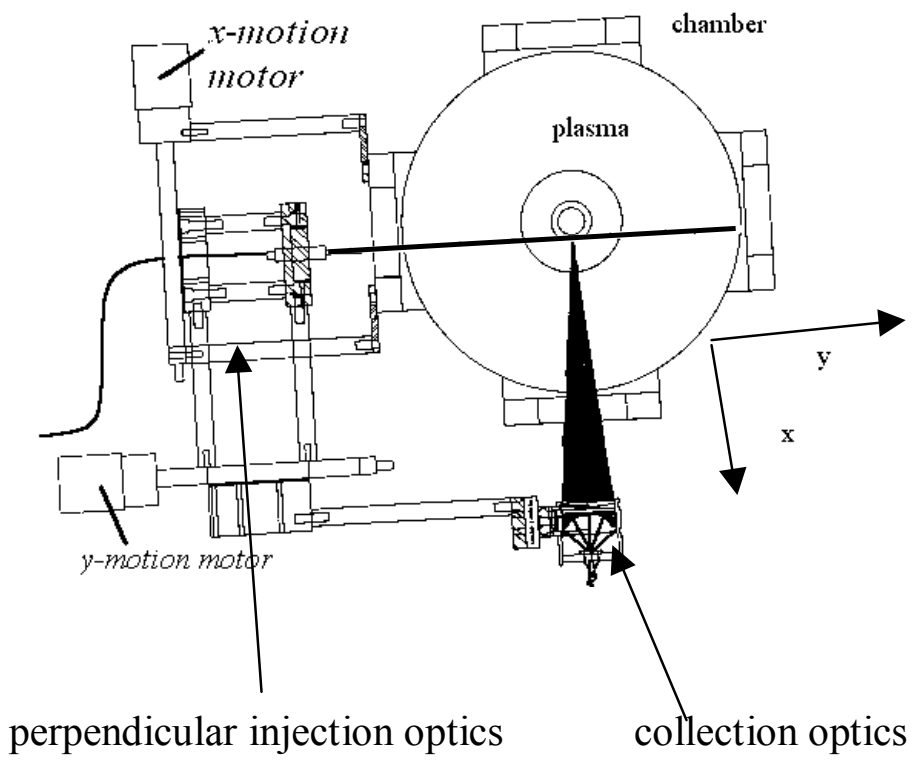

Fig. 13 Perpendicular injection optics and collection optics mounted on the vacuum chamber (reprinted from [Zintl et al., 1999]) 
(a)

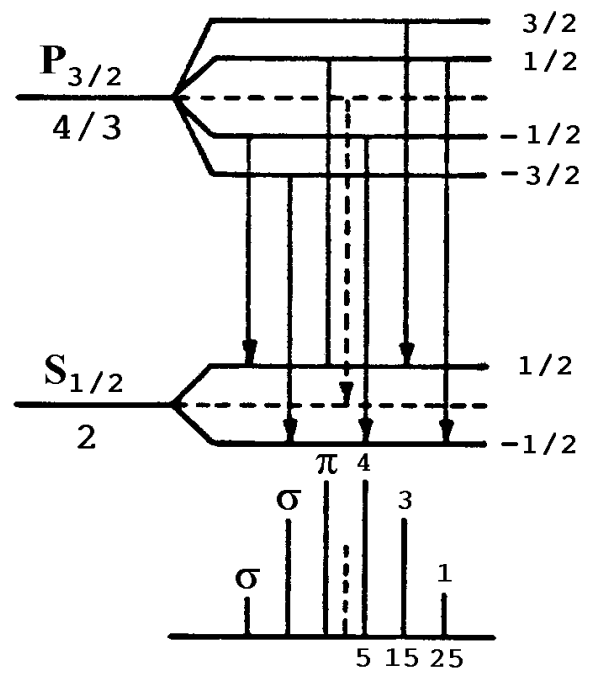

(b)
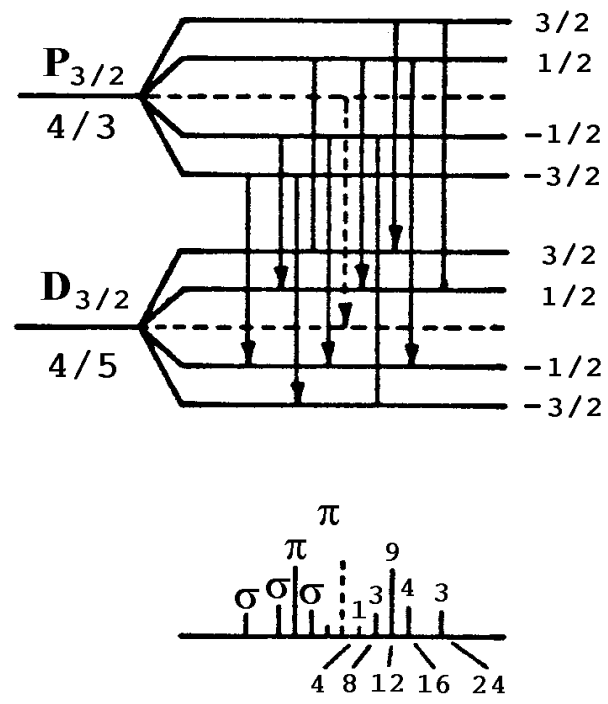

Fig. 14 Grotrian diagram for BaII transitions relevant to the WVU Q plasma machine LIF. Under each energy-level diagram are shown the polarizations of the respective transitions: $\pi$ $\left(\Delta m_{j}=0\right)$ or $\sigma\left(\Delta m_{j}= \pm 1\right)$; the corresponding spectral intensity (upper indices); the corresponding Lande factors $g_{L}$ (lower indices). Fluorescence transitions are represented in (a); optical pumping is achieved through the transitions shown in (b). (reprinted from Hill, [1983]). 


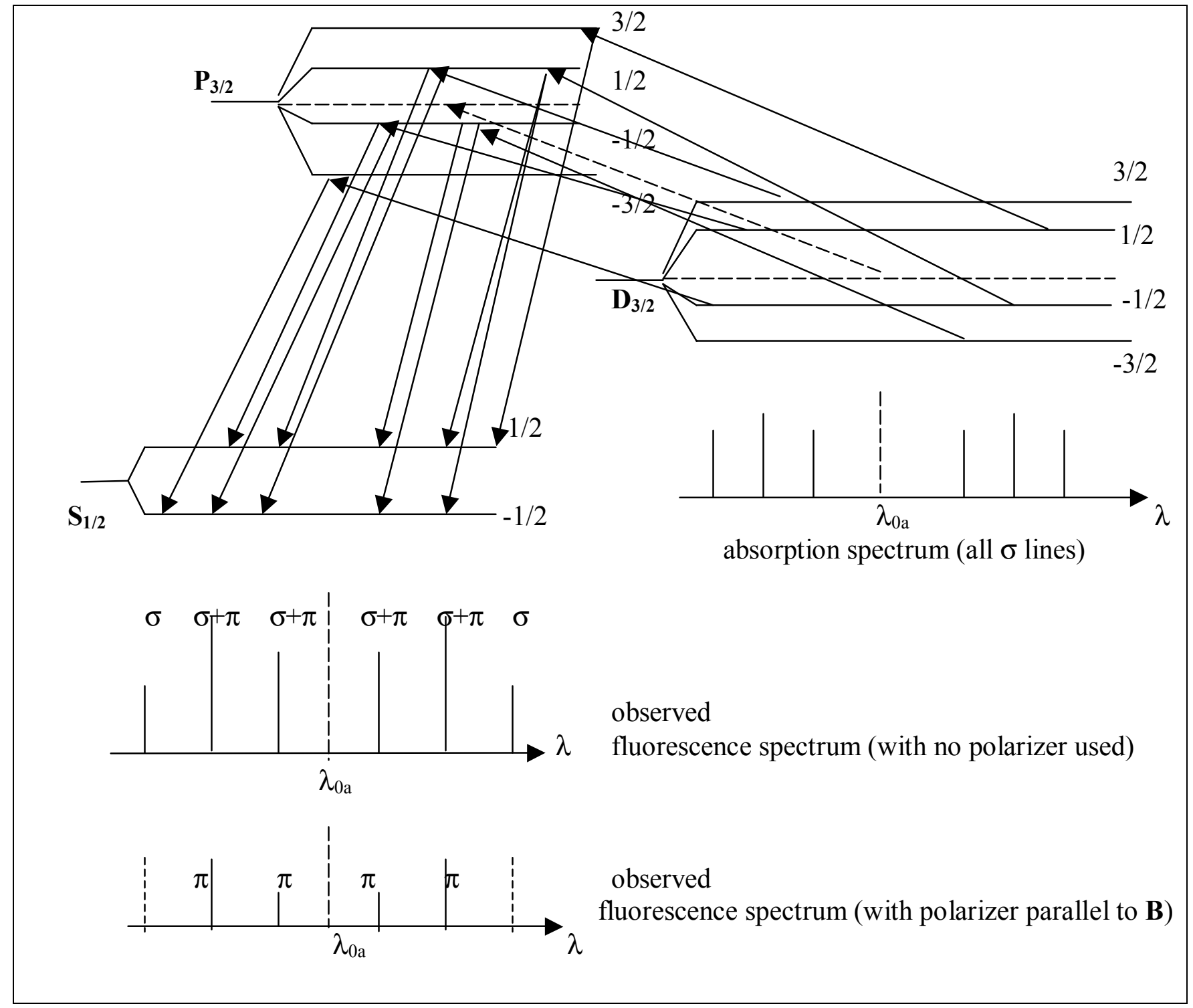

Fig. 15 Laser-induced fluorescence spectrum of barium ions observed when using the parallel injection configuration for the LIF optics. 

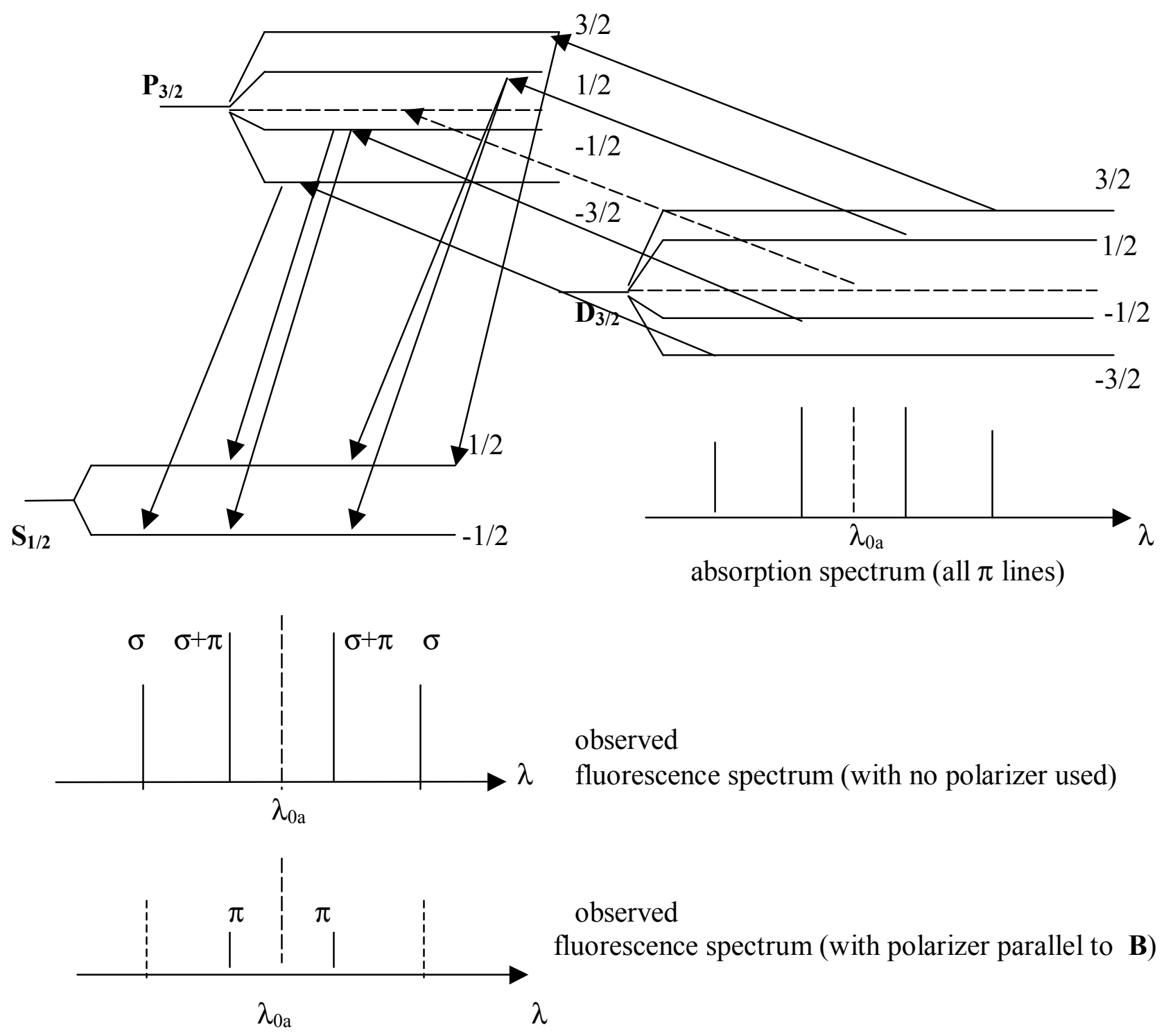

Fig. 16 Laser-induced fluorescence spectrum of barium ions observed when using the perpendicular injection configuration for the LIF optics. 


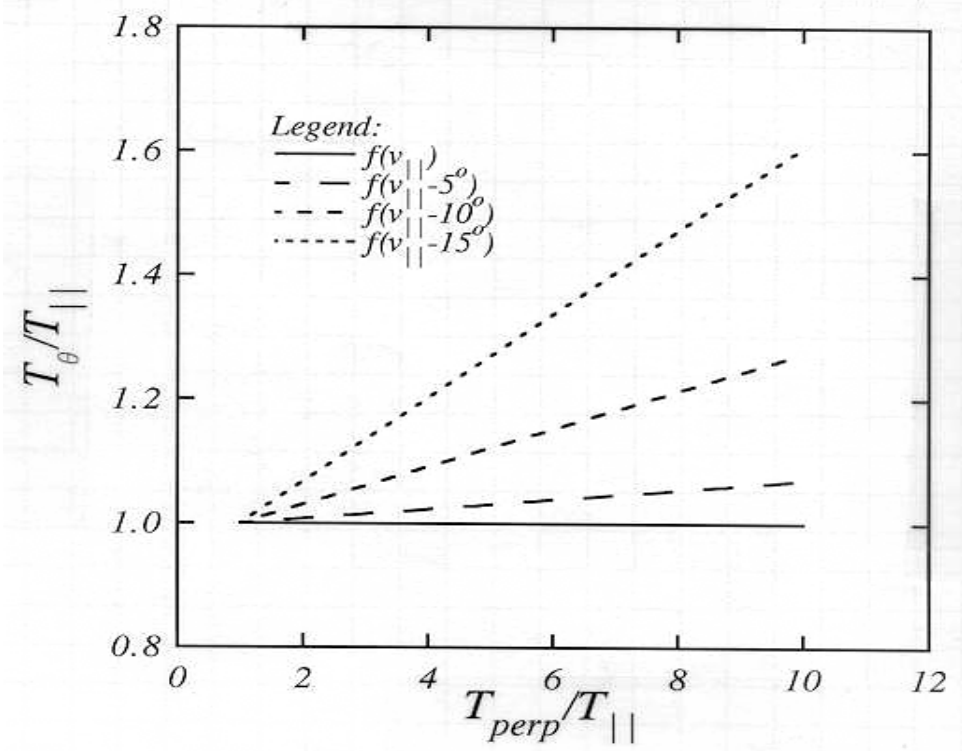

Fig. 17 Parallel LIF-measured plasma temperature as a function of injection angle $\alpha$. (M. Zintl, research notebook, 1999). 


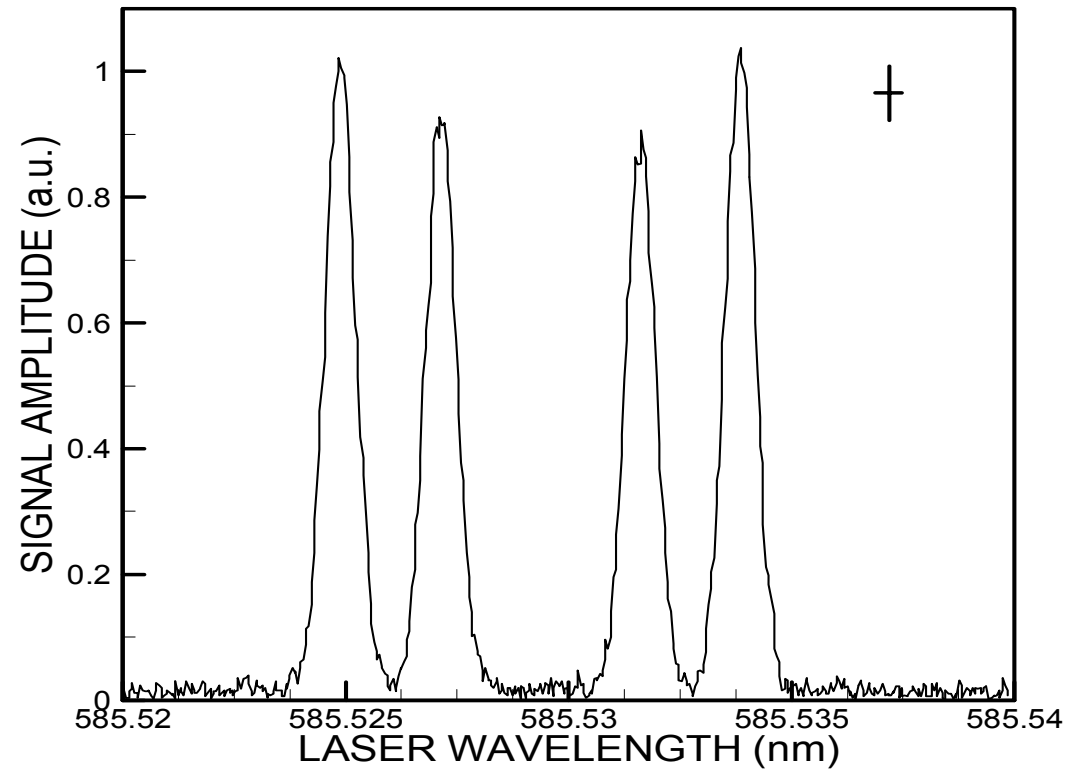

Fig. 18 Fluorescence spectrum ( $\pi$ lines) of cooled-barium plasma using parallel-injection LIF. Here, $T_{i z}=0.034 \mathrm{eV}$. (data acquired and analyzed by E. Reynolds, Ph.D. dissertation). 

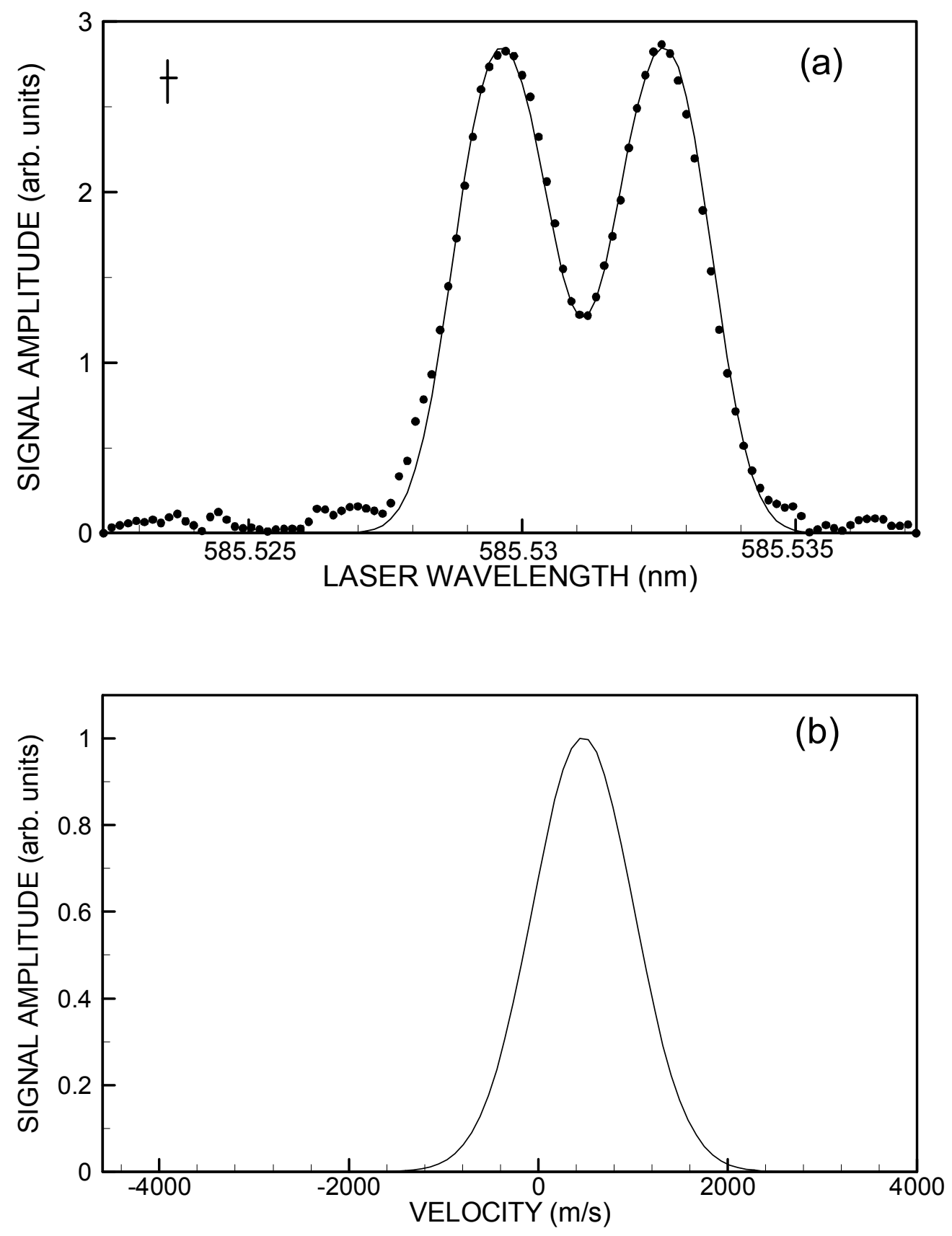

Fig. 19 (a) Parallel-LIF spectrum (dots) and the best-fit lineshape; (b) the corresponding single drifting Maxwellian distribution obtained from the fitted lineshape. 

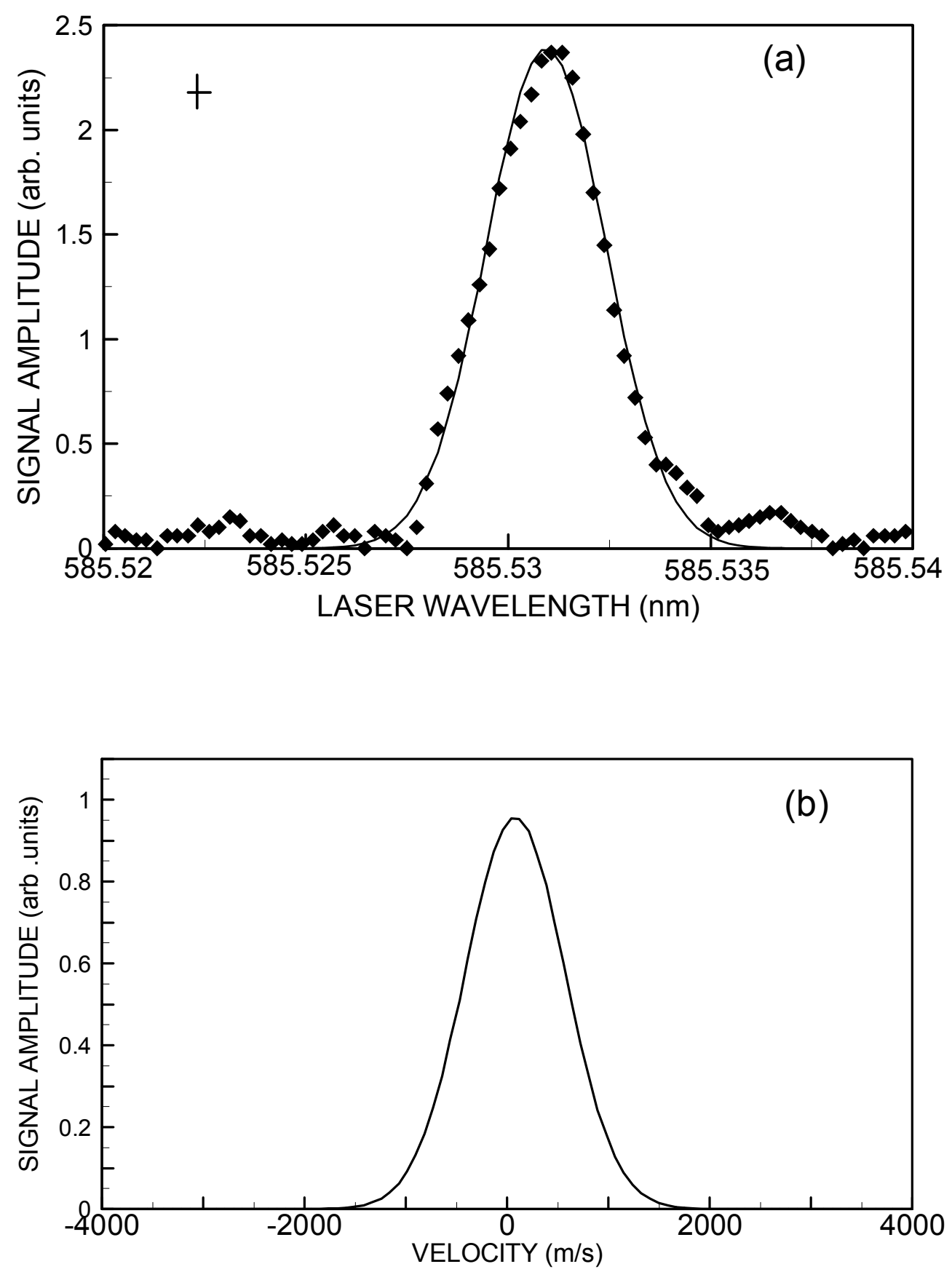

Fig. 20 (a) Perpendicular-LIF spectrum (dots) and the best-fit lineshape; (b) the corresponding single drifting-Maxwellian distribution obtained from the fitted lineshape. 

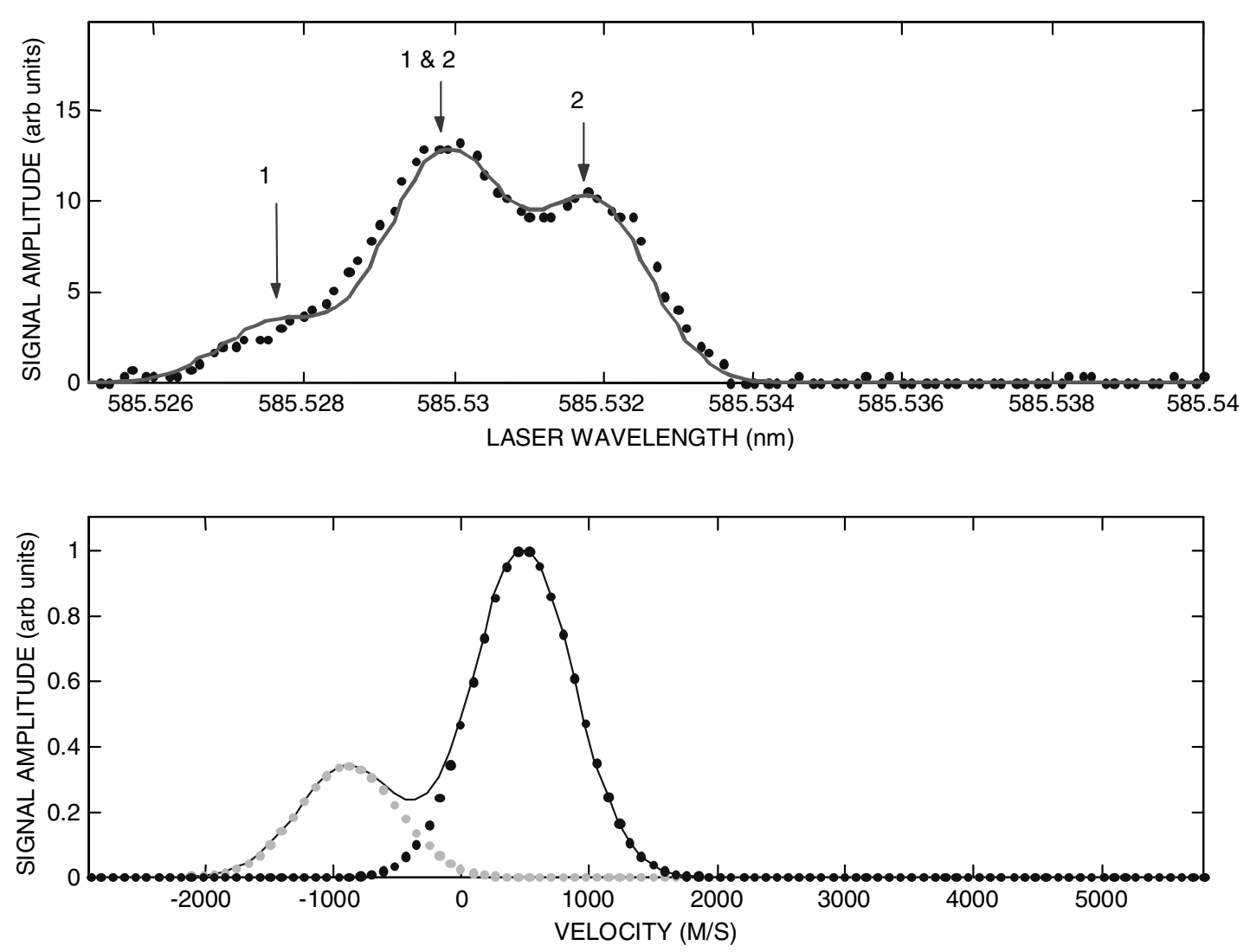

Fig. 21 (a) Parallel LIF-measured barium spectrum (dots) and the best-fit lineshape; (b) the corresponding two drifting-Maxwellian distributions obtained from the fitted lineshape and their sum (line). 

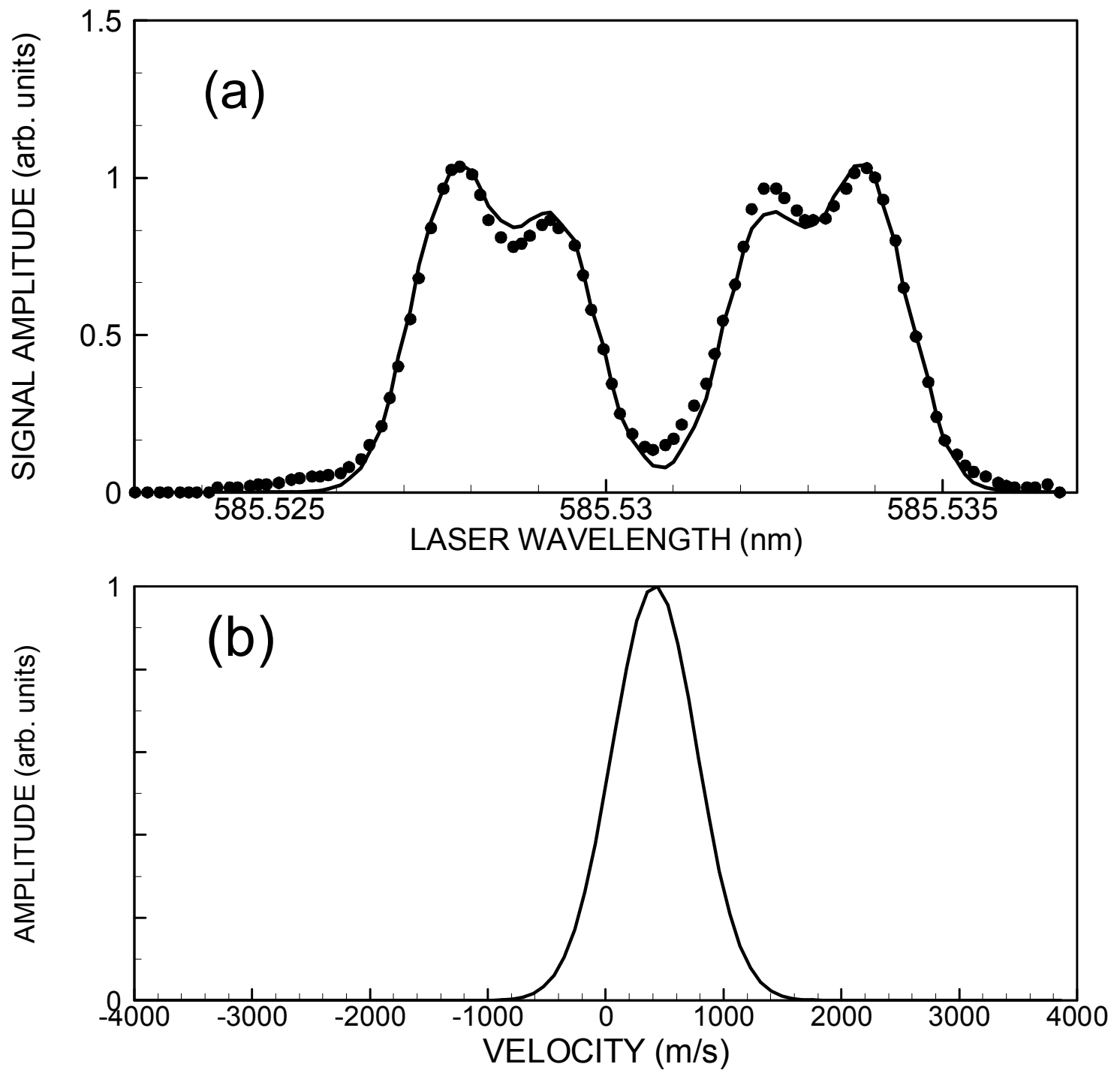

Fig. 22 (a) Parallel-LIF-measured barium spectrum (dots) and the best-fit lineshape in the Q machine in SISE configuration. (b) The corresponding single drifting Maxwellian velocity distribution. 


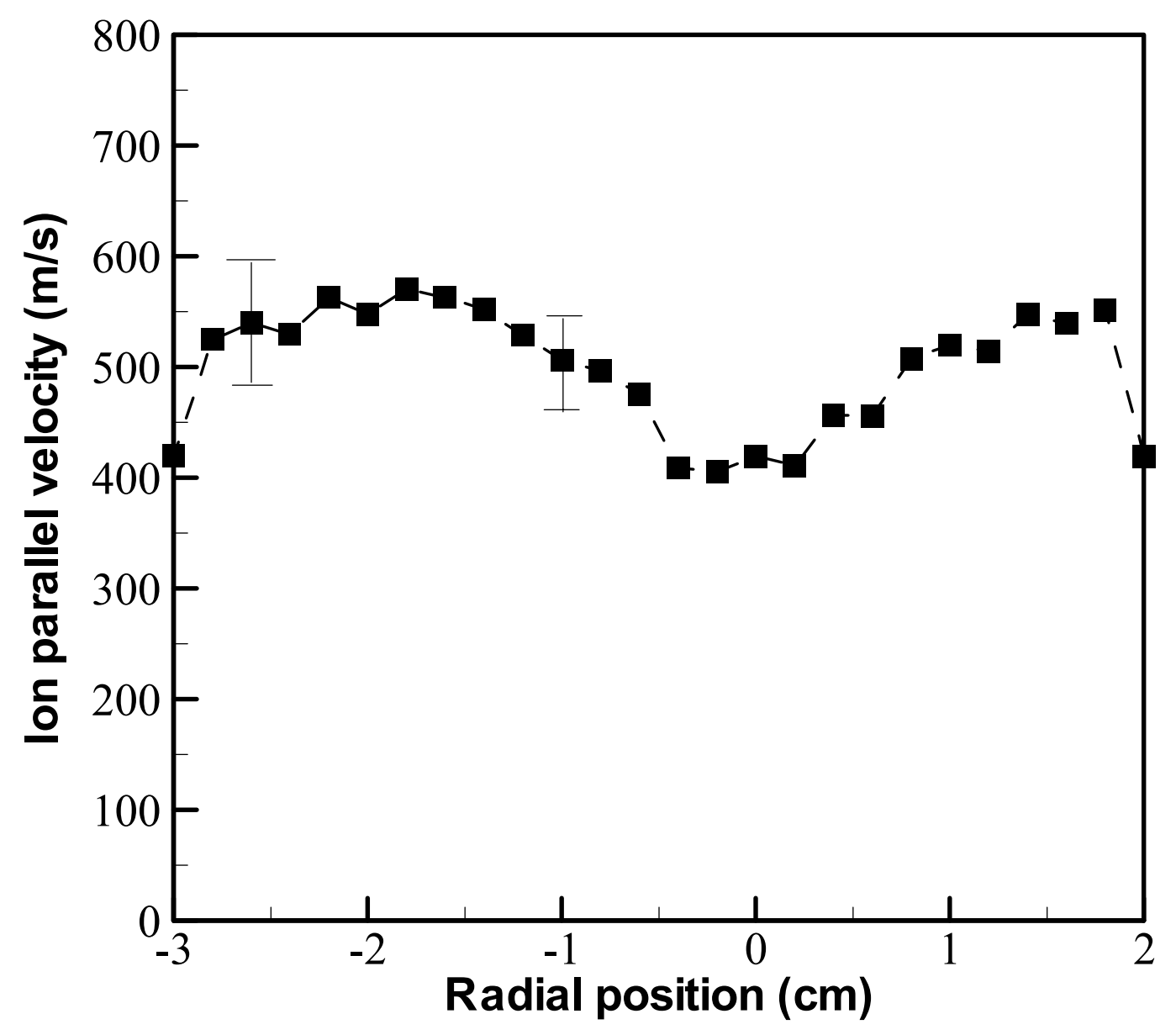

Fig. 23 Inhomogeneous parallel ion drift velocity radial profile obtained using the annular electrode biased at $V_{A}=35 \mathrm{~V}$ and the termination plate biased at $V_{T}=-5 \mathrm{~V}$. 


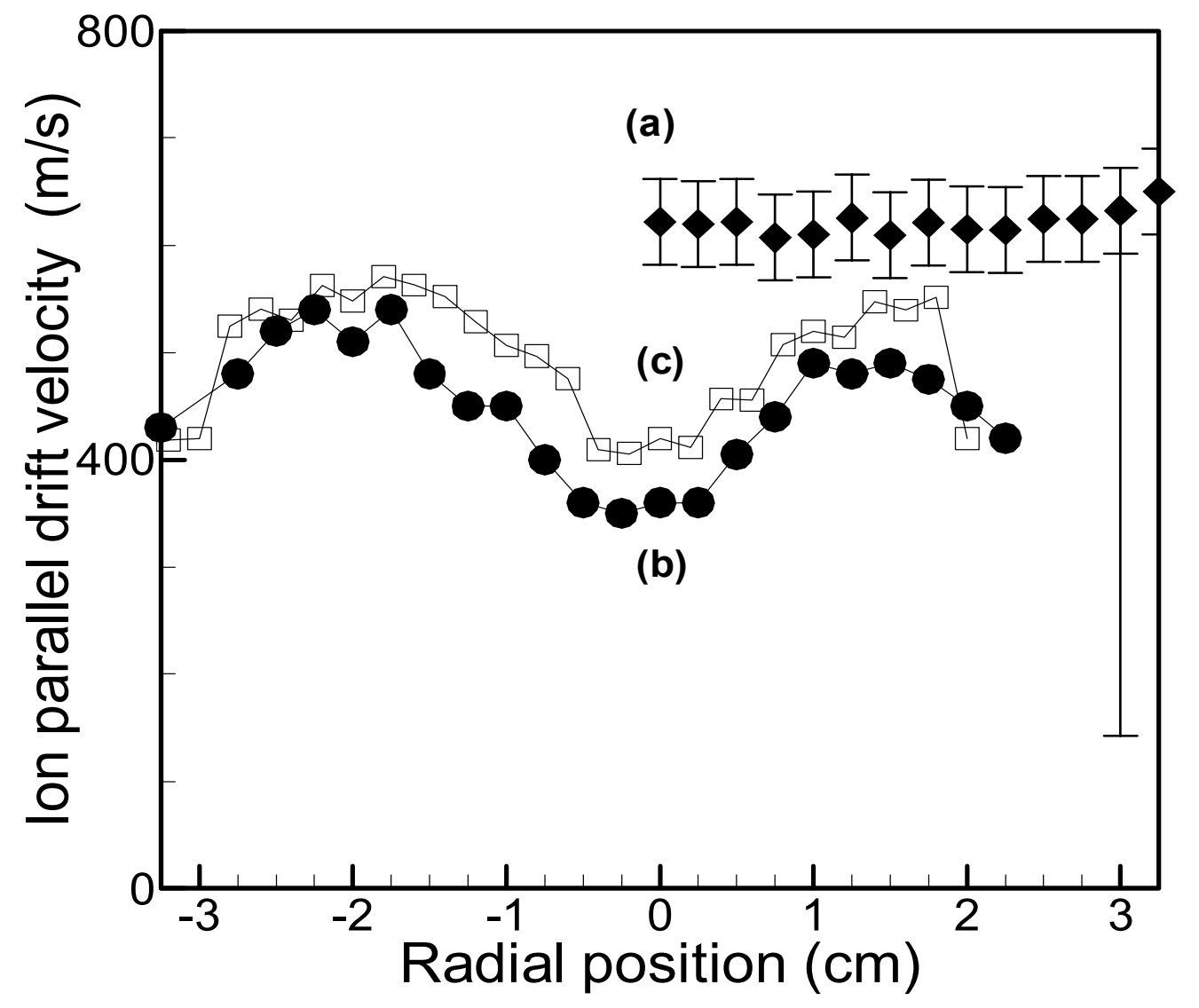

Fig. 24 Laser-induced fluorescence measurement of the radial profile of barium-ion parallel drift velocity, representative of (a) negligible shear using either annular or button electrode (here, button with $V_{0}=20 \mathrm{~V}, V_{\mathrm{T}}=0,1.4 \mathrm{kG}$ ), (b) nonzero shear using annulus $\left(V_{0}=35 \mathrm{~V}, V_{\mathrm{T}}=0,2 \mathrm{kG}\right)$, and (c) nonzero shear using button $\left(V_{0}=35 \mathrm{~V}, V_{\mathrm{T}}=0,2 \mathrm{kG}\right)$. The long error bar at the right of (a) represents the ion thermal speed for all data (i.e., $T_{\mathrm{i}}$ is uniform). 


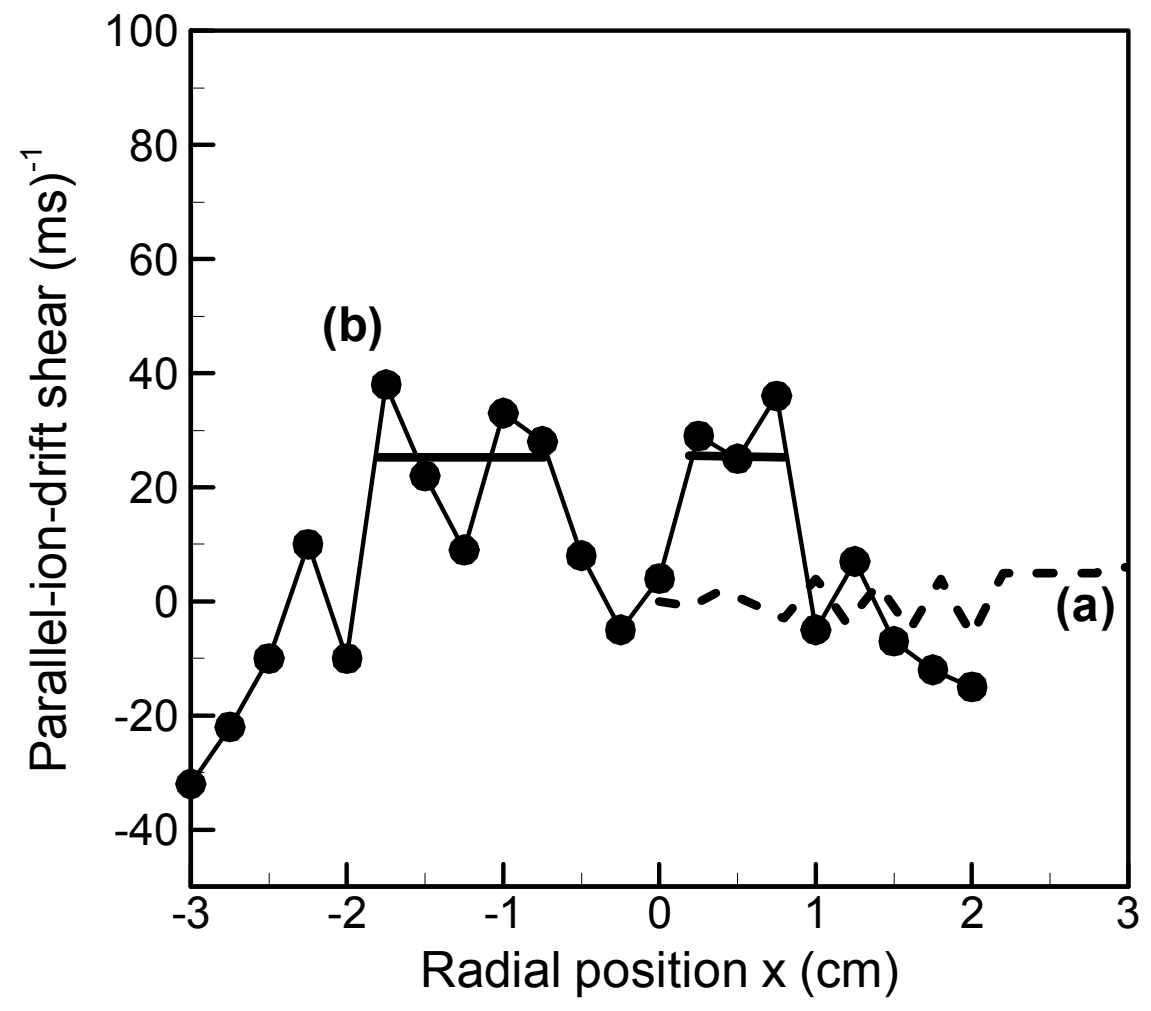

Fig. 25 Parallel-ion-drift-velocity shear as a function of radial position associated with velocity profiles (a) and (b) in Fig. 24.

Multi-point average value of shear is shown by the two horizontal lines. 

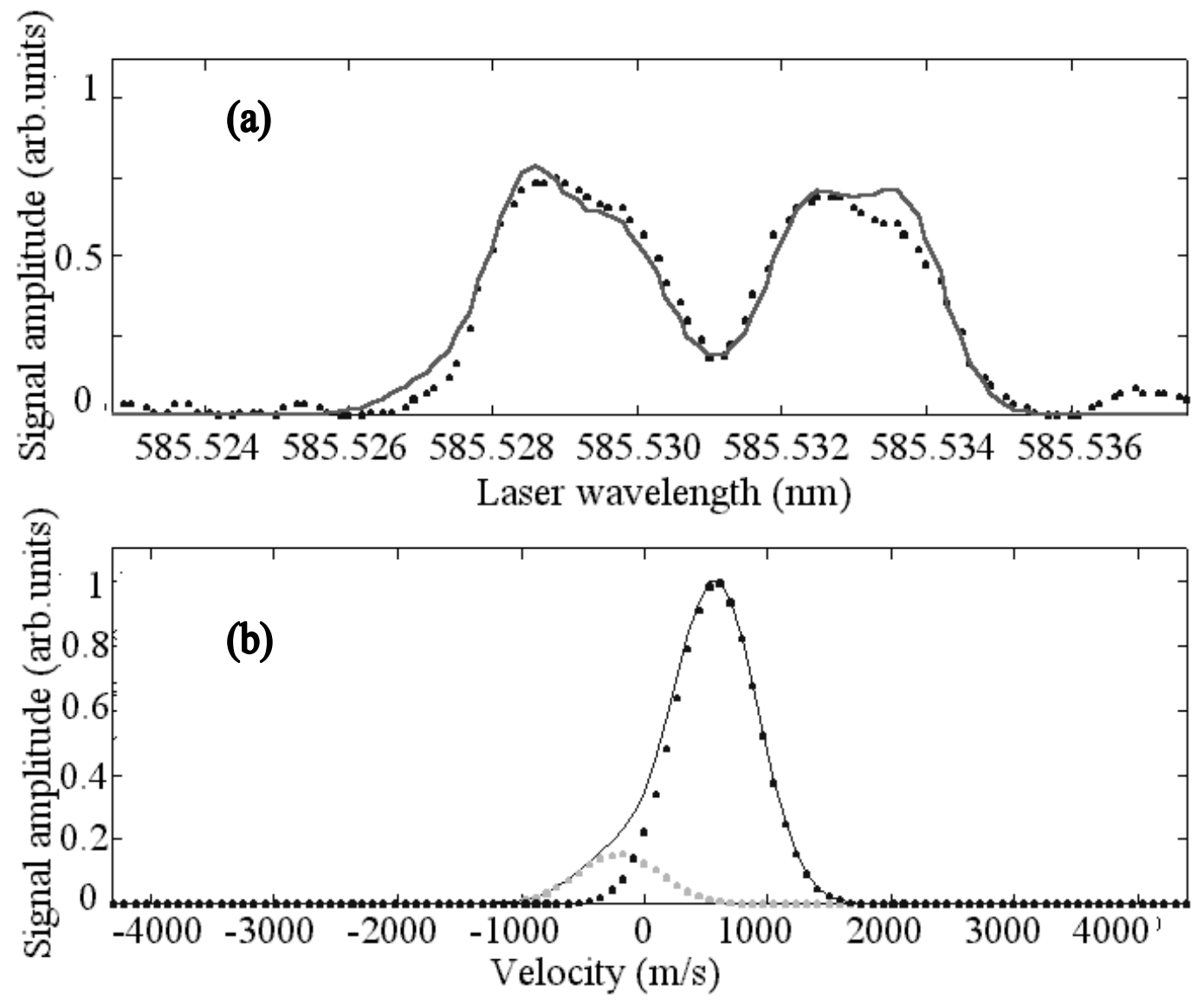

Fig. 26 (a) LIF-measured barium-ion spectrum spectrum (dots) and the best-fit lineshape. (b) The corresponding two drifting-Maxwellian ion velocity distributions. 

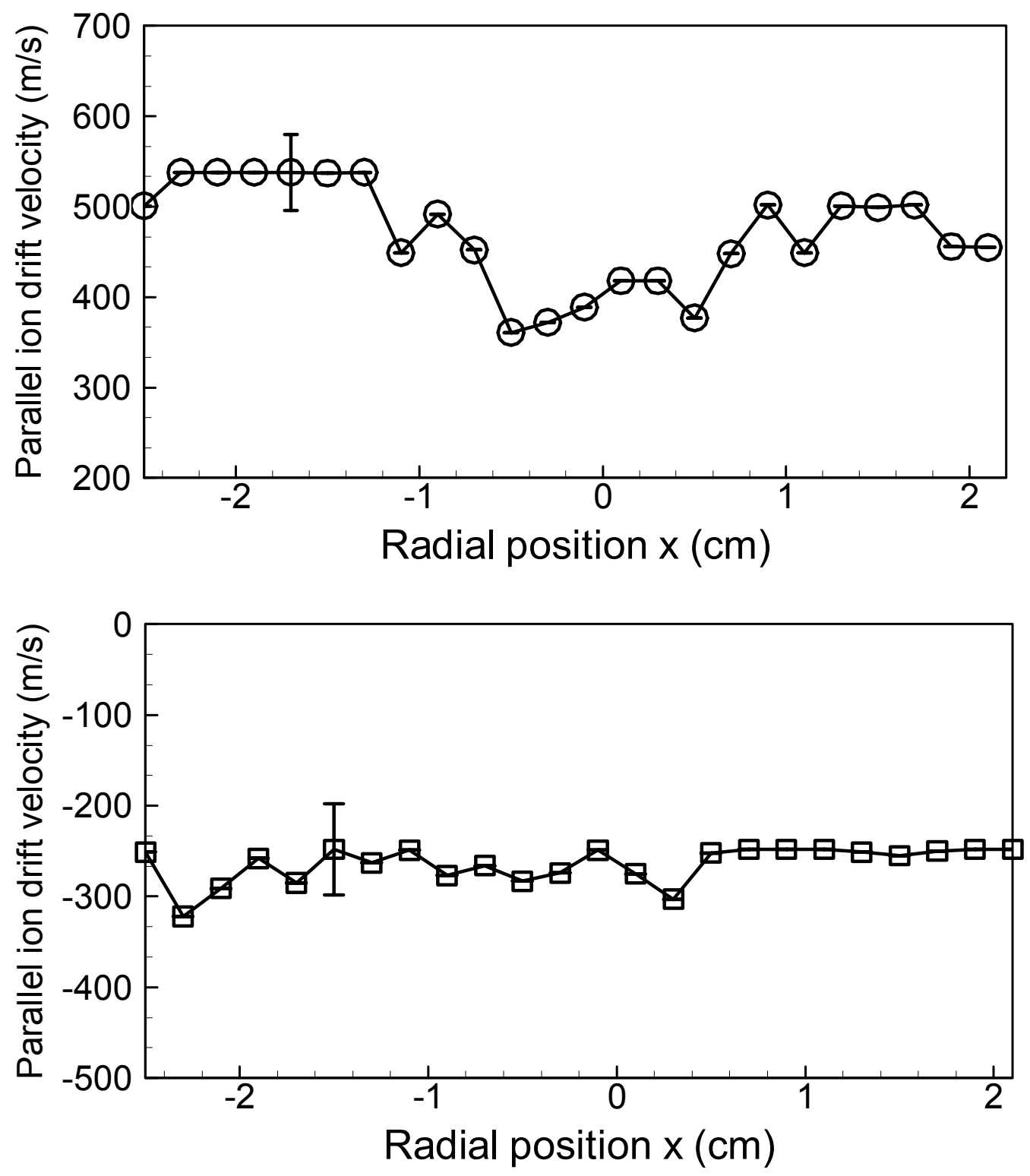

Fig. 27 (a) Parallel ion drift velocity radial profiles of the direct (parallel) population and of the counter-streaming (anti-parallel) population (b) when two Maxwellian populations are present. Parallel-progating population is 6 times denser than the anti-parallel-propagating population. 


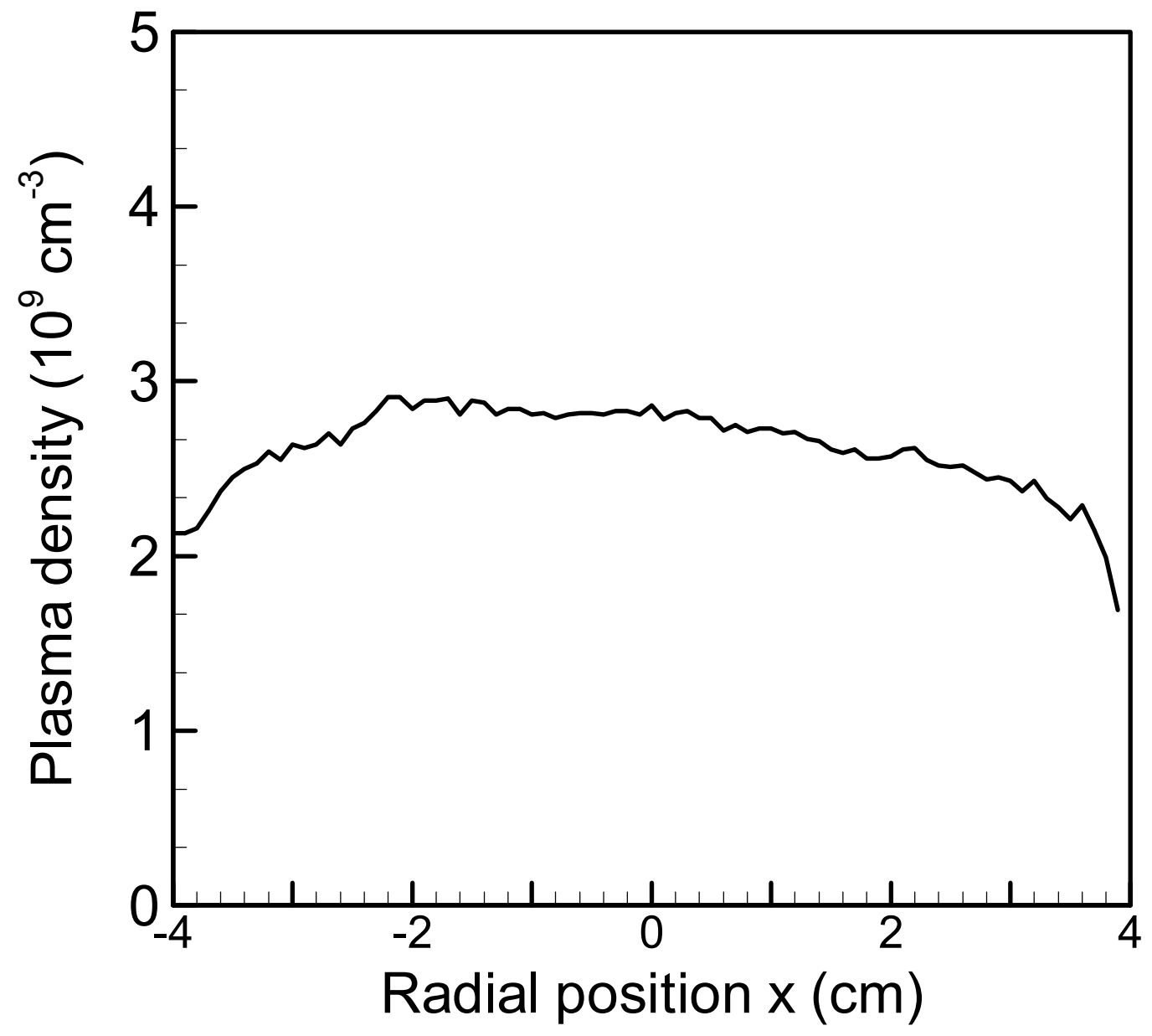

Fig. 28 Plasma density radial profile in the SISE configuration of the Q machine, inferred from ion saturation current measurements. 


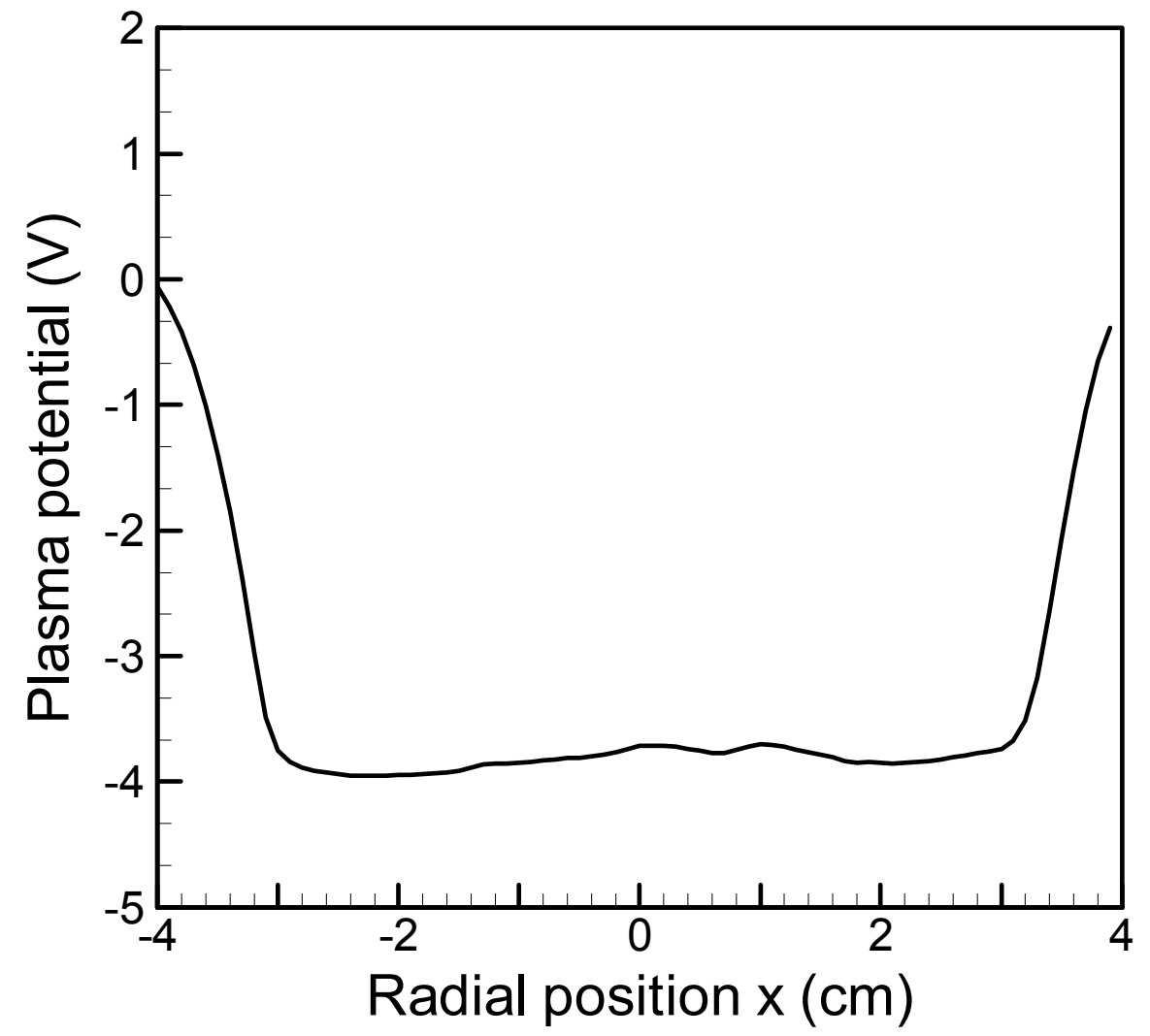

Fig. 29 Plasma potential radial profile in the SISE configuration $(z=69 \mathrm{~cm})$. A biased $\left(V_{A}=35 \mathrm{~V}\right)$ annular electrode $(3.6 \mathrm{~cm}$ outer diameter, $1.75 \mathrm{~cm}$ inner diameter) is located on the cylindrical axis with the surface normal aligned with the magnetic field. 


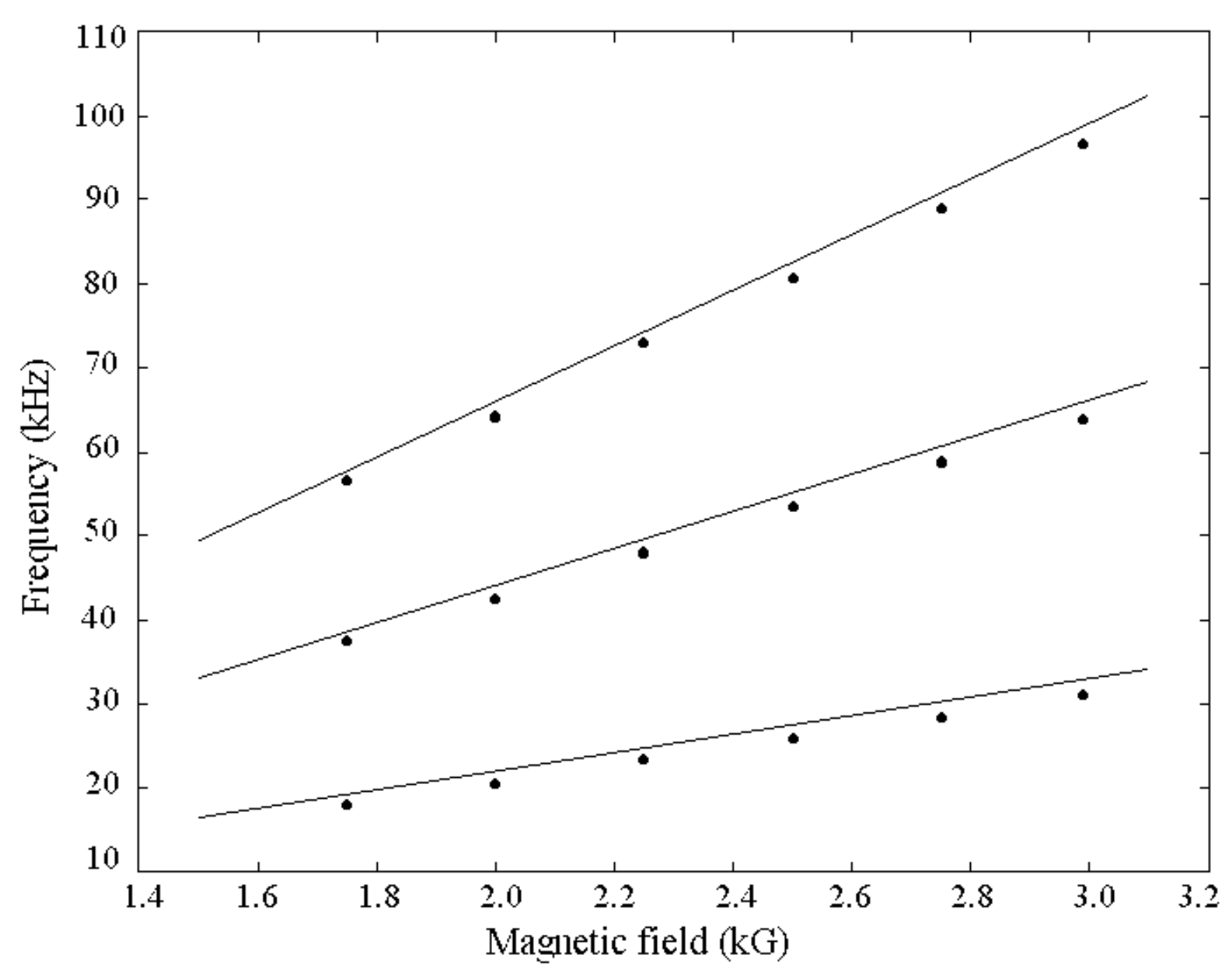

Fig. 30 Magnetic field strength dependence of the frequency multi-harmonic ion cyclotron waves in the presence of inverse ion cyclotron damping. Straight lines are harmonics of the ion gyrofrequency. 

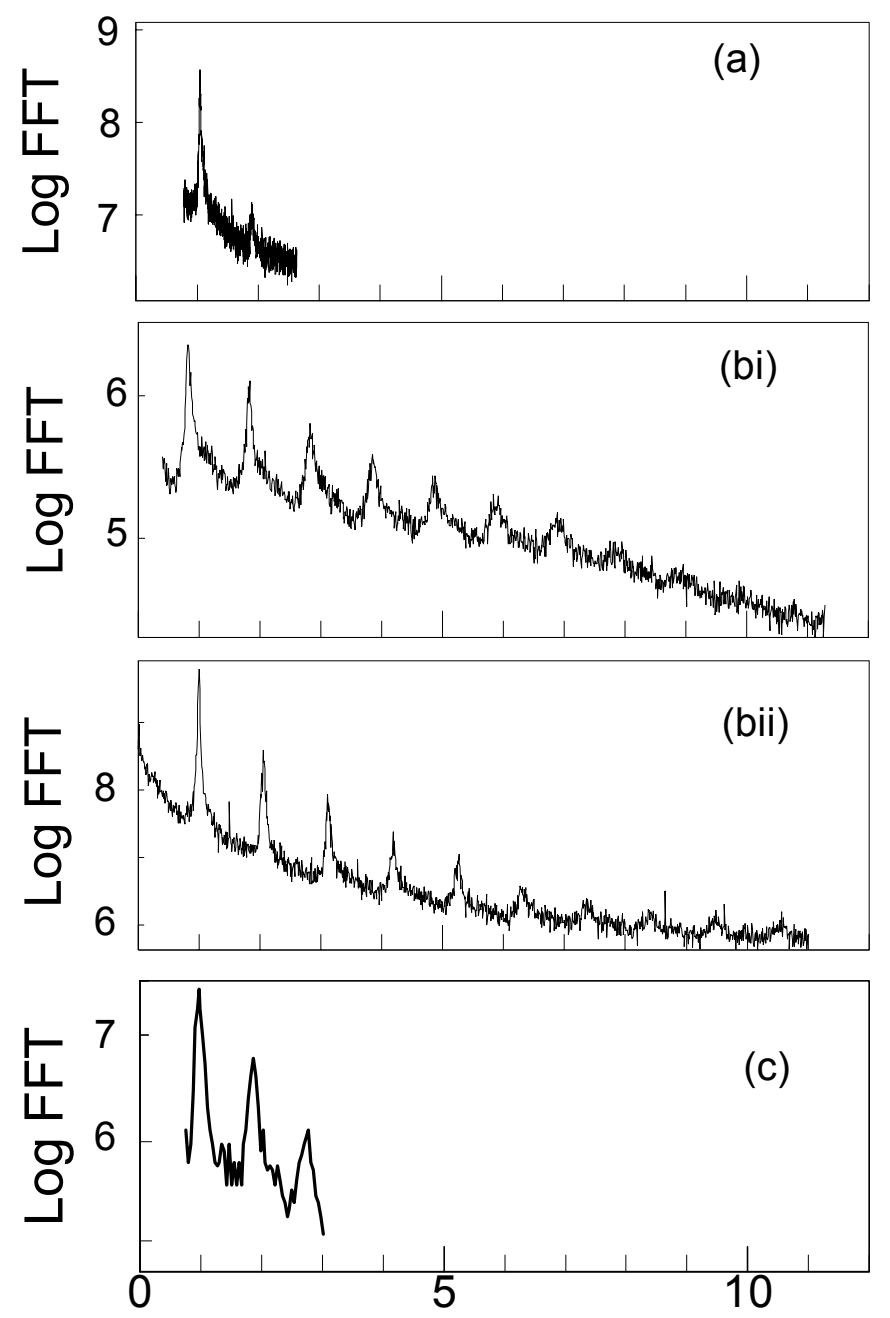

Normalized frequency

Fig. 31 Spectra of ion saturation current fluctuations collected by (a) the annular electrode (negligible-shear case, $V_{0}=15 \mathrm{~V}, V_{\mathrm{T}}=0,2 \mathrm{kG}$ ), (bi) the annular electrode (nonzero-shear case, same as Fig. 24b), (bii) the Langmuir probe (same as previous case (bi)), and (c) the button electrode (nonzero-shear case, same as Fig. 24c). 


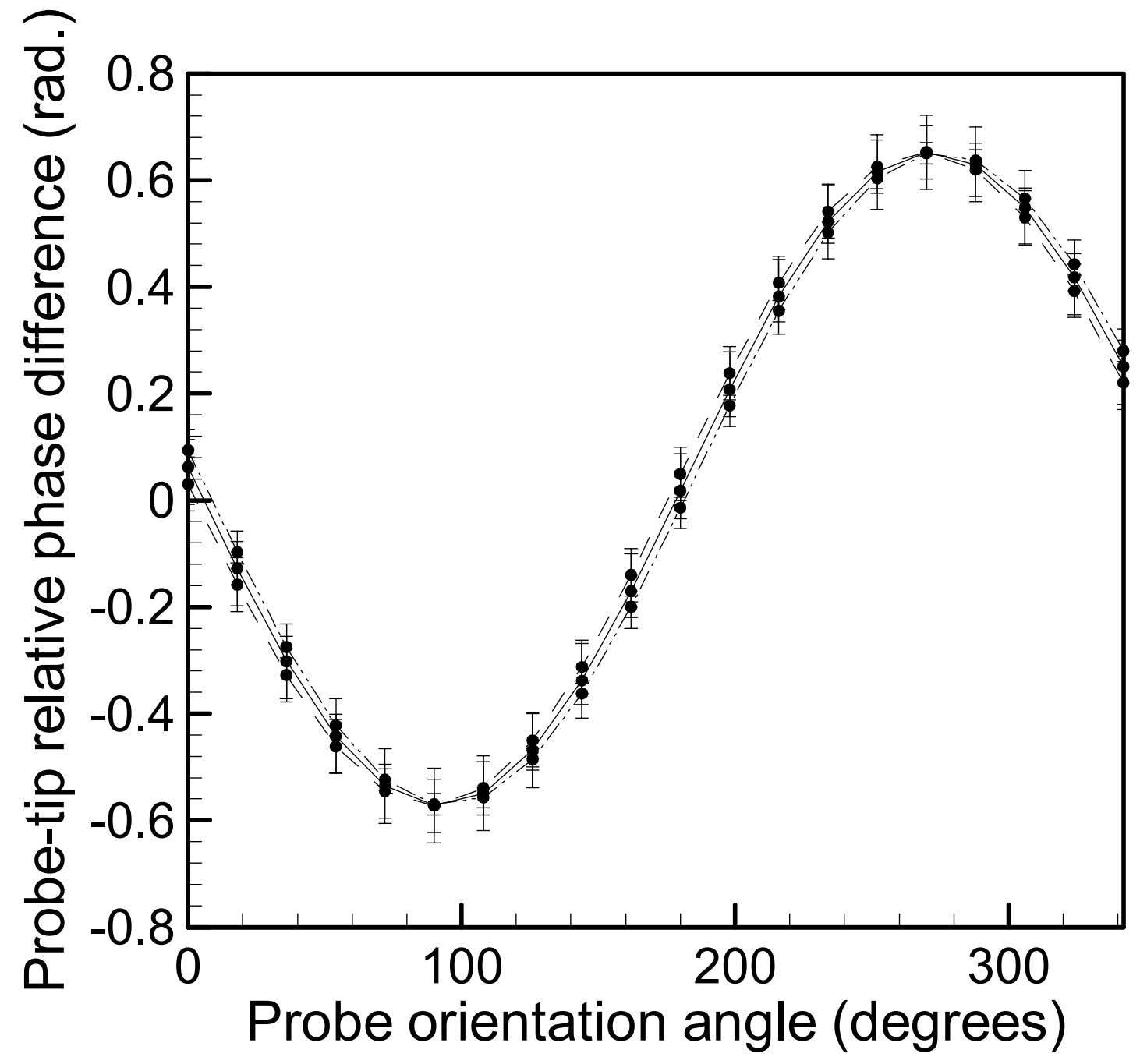

Fig. 32 Probe-tip phase difference as a function of probe orientation and the best fitted sinusoid (black) yielding $k_{y}=-2.120 \mathrm{~cm}^{-1}, k_{z}=0.024 \mathrm{~cm}^{-1}$. The dashed line is a sinusoid with $k_{y}=-2.080 \mathrm{~cm}^{-1}$, $k_{z}=0.019 \mathrm{~cm}^{-1}$, the dot-dashed one has $k_{y}=-2.160 \mathrm{~cm}^{-1}, k_{z}=0.029 \mathrm{~cm}^{-1}$, representing the mean value plus/minus the standard deviation. 


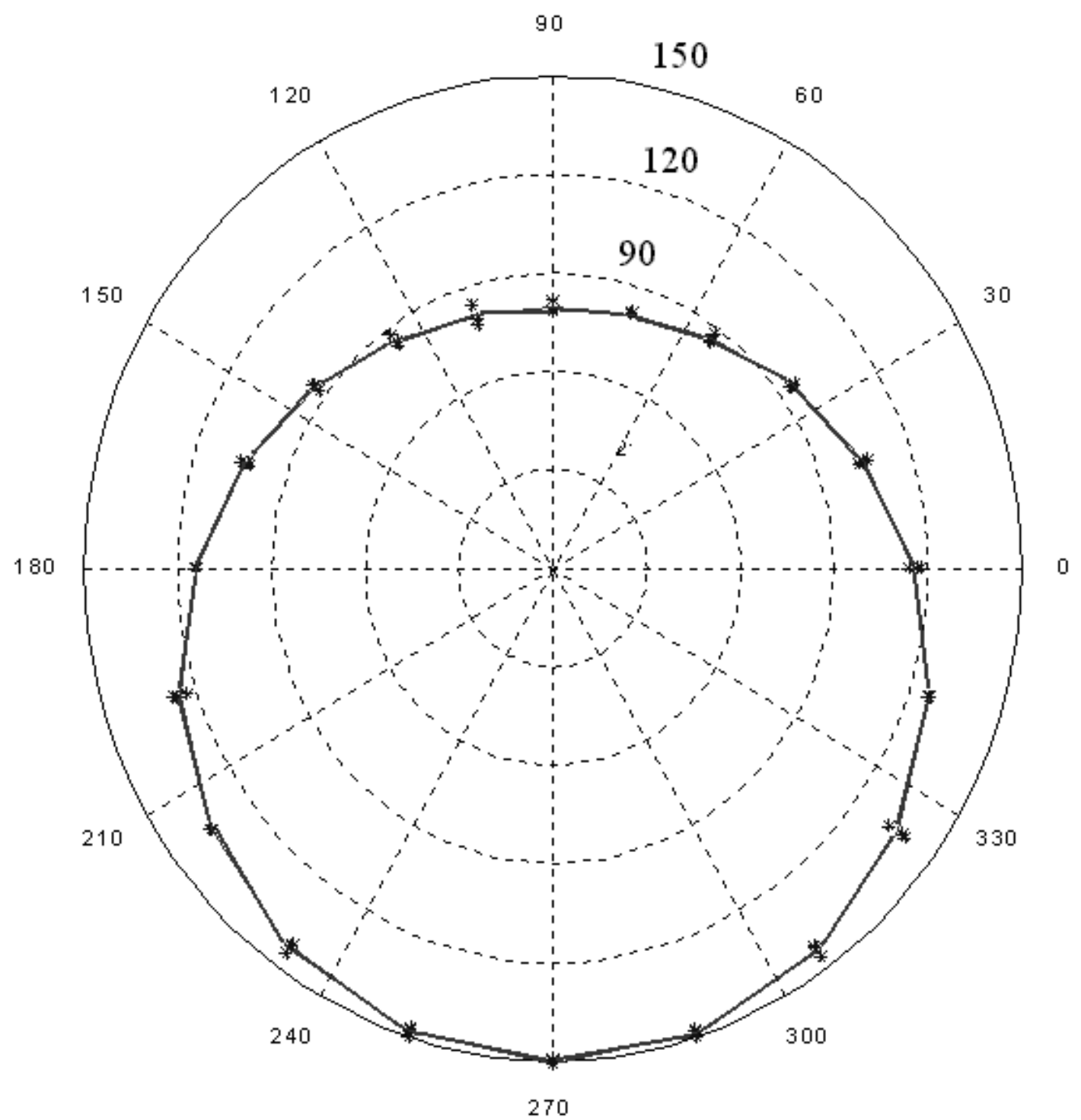

Fig. 33 The circle method applied to data shown in Fig. 32. Wavevector components are $k_{z}=(0.024 \pm 0.003) \mathrm{cm}^{-1}$ and $k_{y}=(-2.120 \pm 0.020) \mathrm{cm}^{-1}$. 


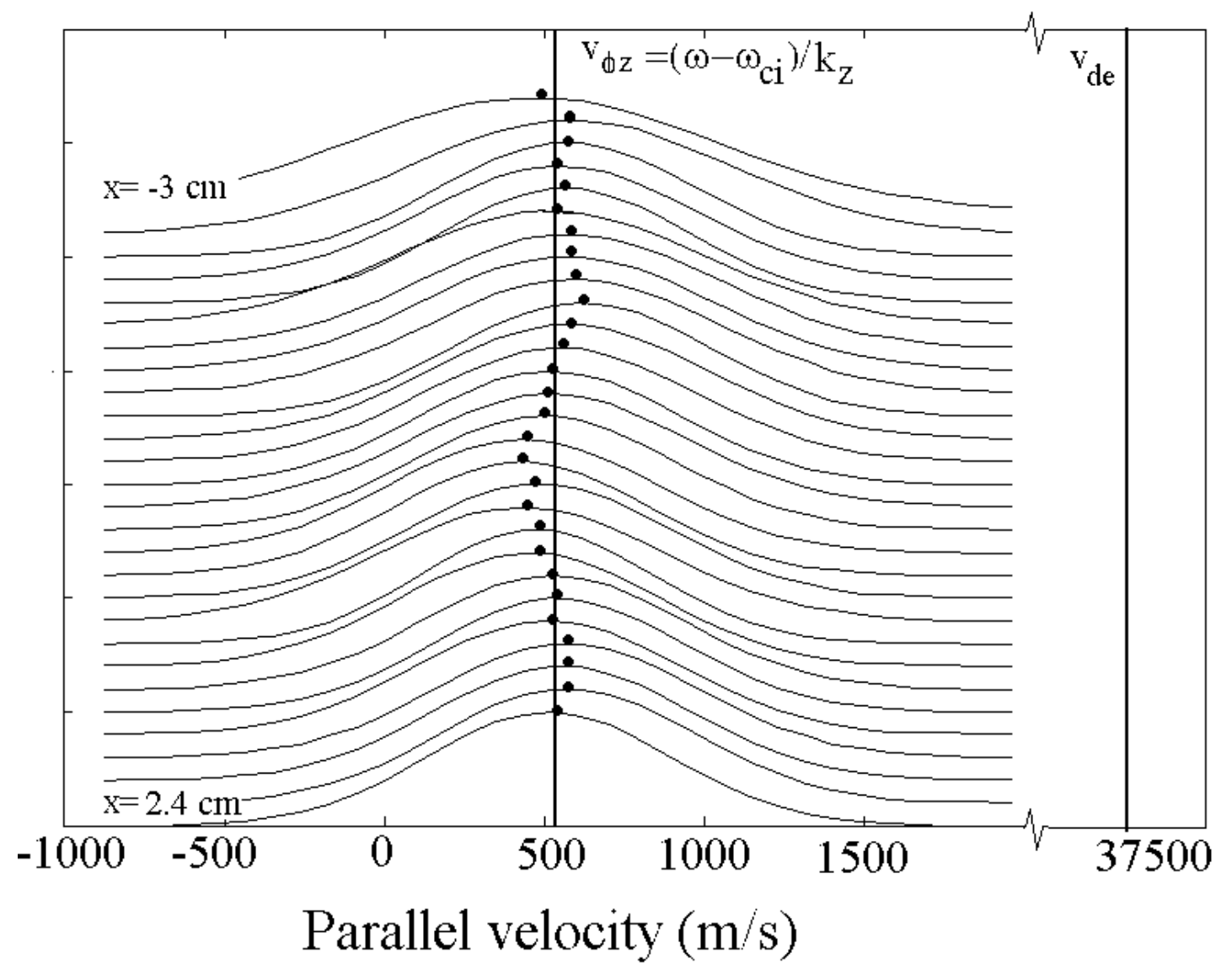

Fig. 34 Intersection of ion-gyroframe-SMIC-wave parallel phase velocity with the ion parallel-velocity distribution. Dots indicate the local values of the parallel-ion-drift velocity. The $x$ spacing between the velocity distributions is $0.2 \mathrm{~cm}$. 


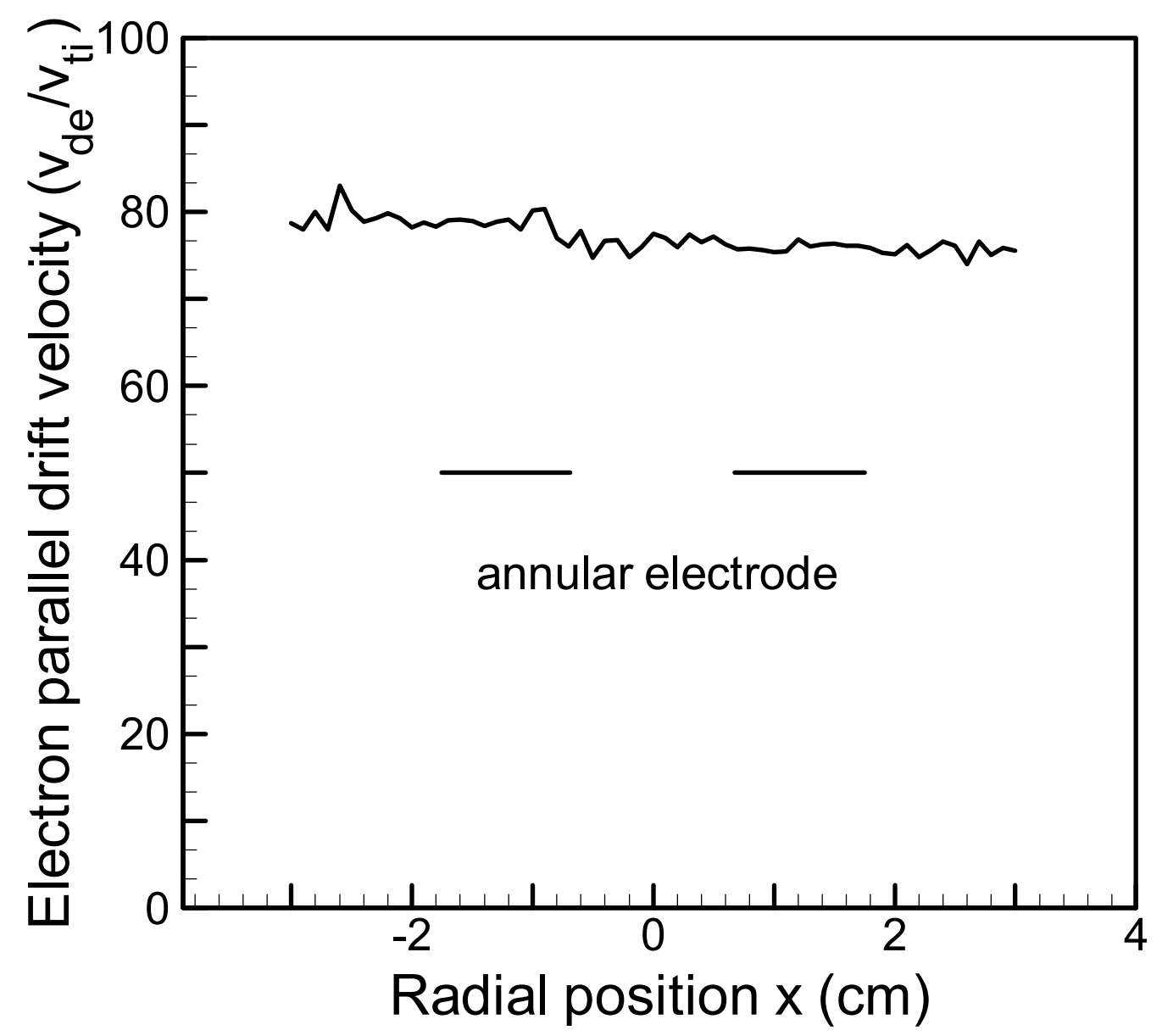

Fig. 35 Parallel electron drift velocity radial profile in the presence of parallel-ionvelocity shear and multi-harmonic SMIC waves. No structure in the parallel velocity profile is observed to correspond with the radial position of the electrode. Here, $v_{t i}=544 \mathrm{~m} / \mathrm{s}$. 


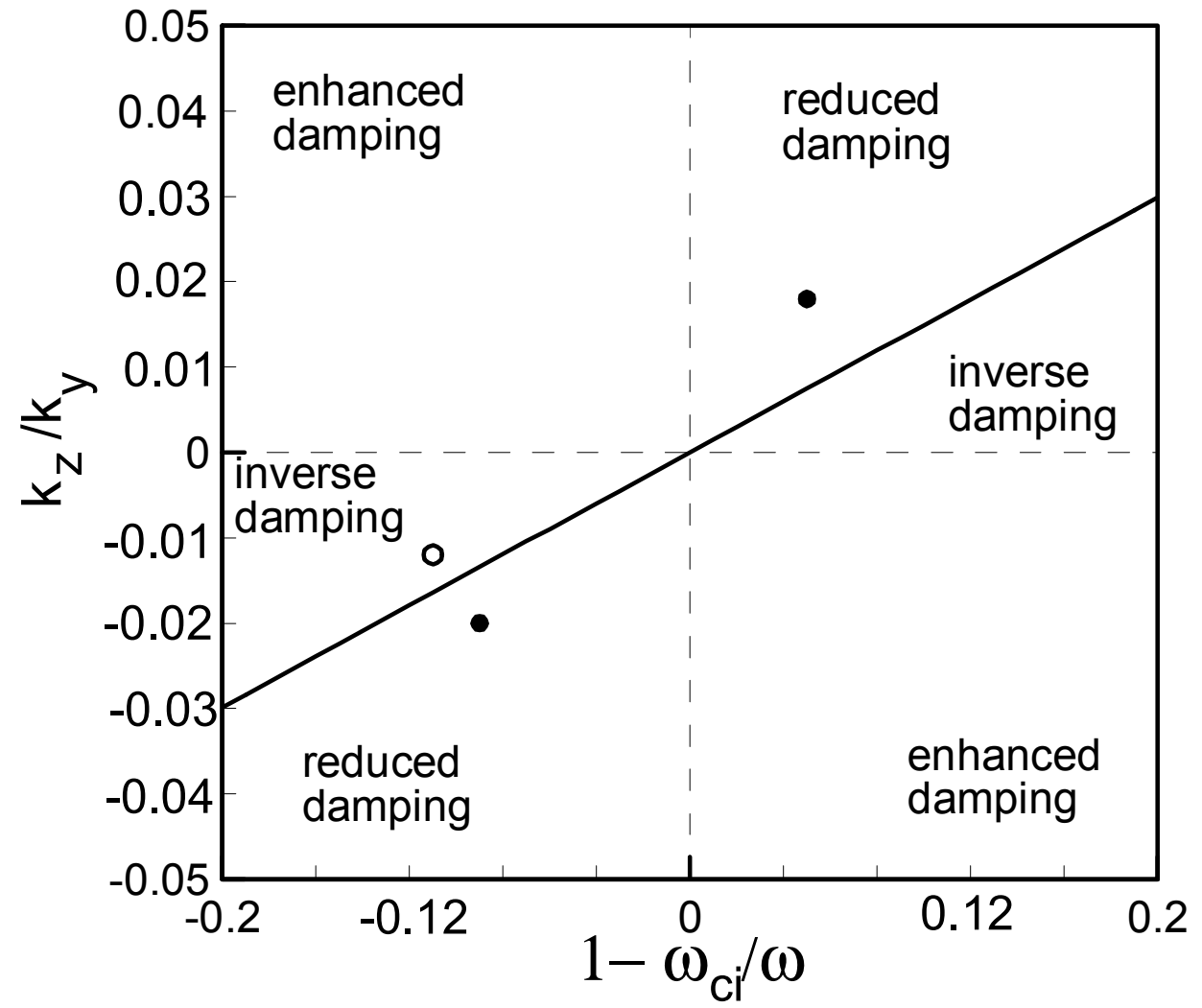

Fig. 36 SMIC wave regimes for fixed shear $d v / d x=0.14 \omega_{c i}$. The three points represent experimental data points. The straight line is the locus of points for which the ion cyclotron damping factor is equal to zero. 


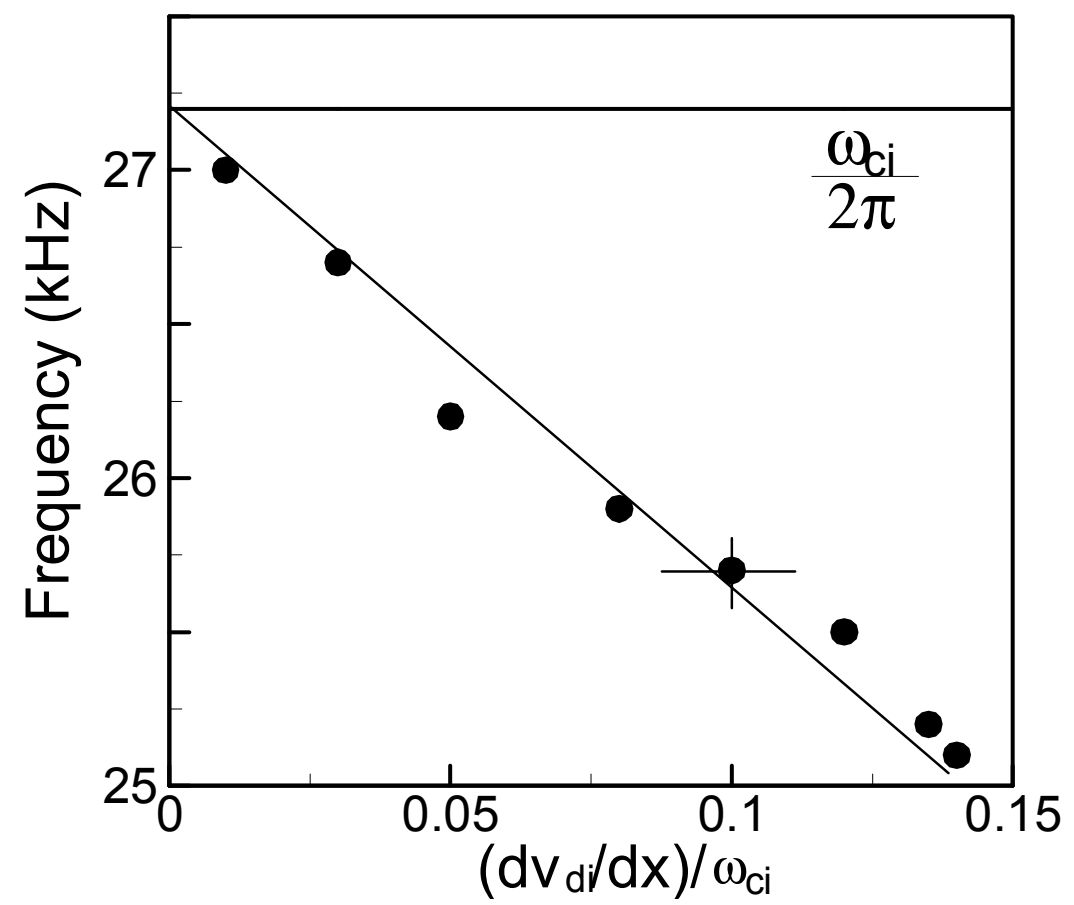

Fig. 37 Dependence of multi-harmonic SMIC wave fundamental frequency on shear value. 


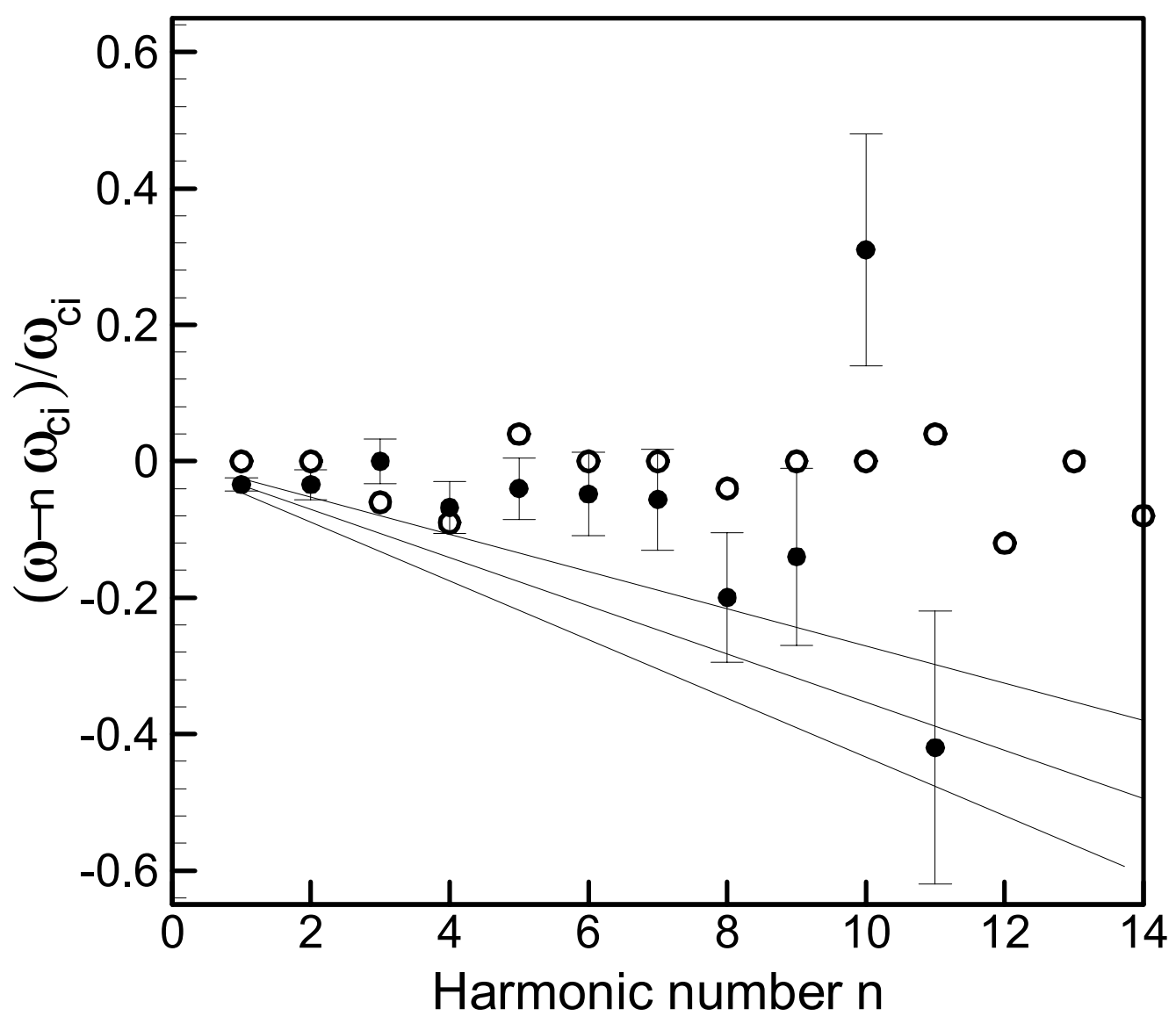

Fig. 38 Multi-harmonic SMIC waves normalized frequency as a function of harmonic number (filled circles). Linear-theory-predicted wave frequency (open circles) for shear-driven multi-harmonic EIC waves (from [Gavrishchaka et al., 2000]). 


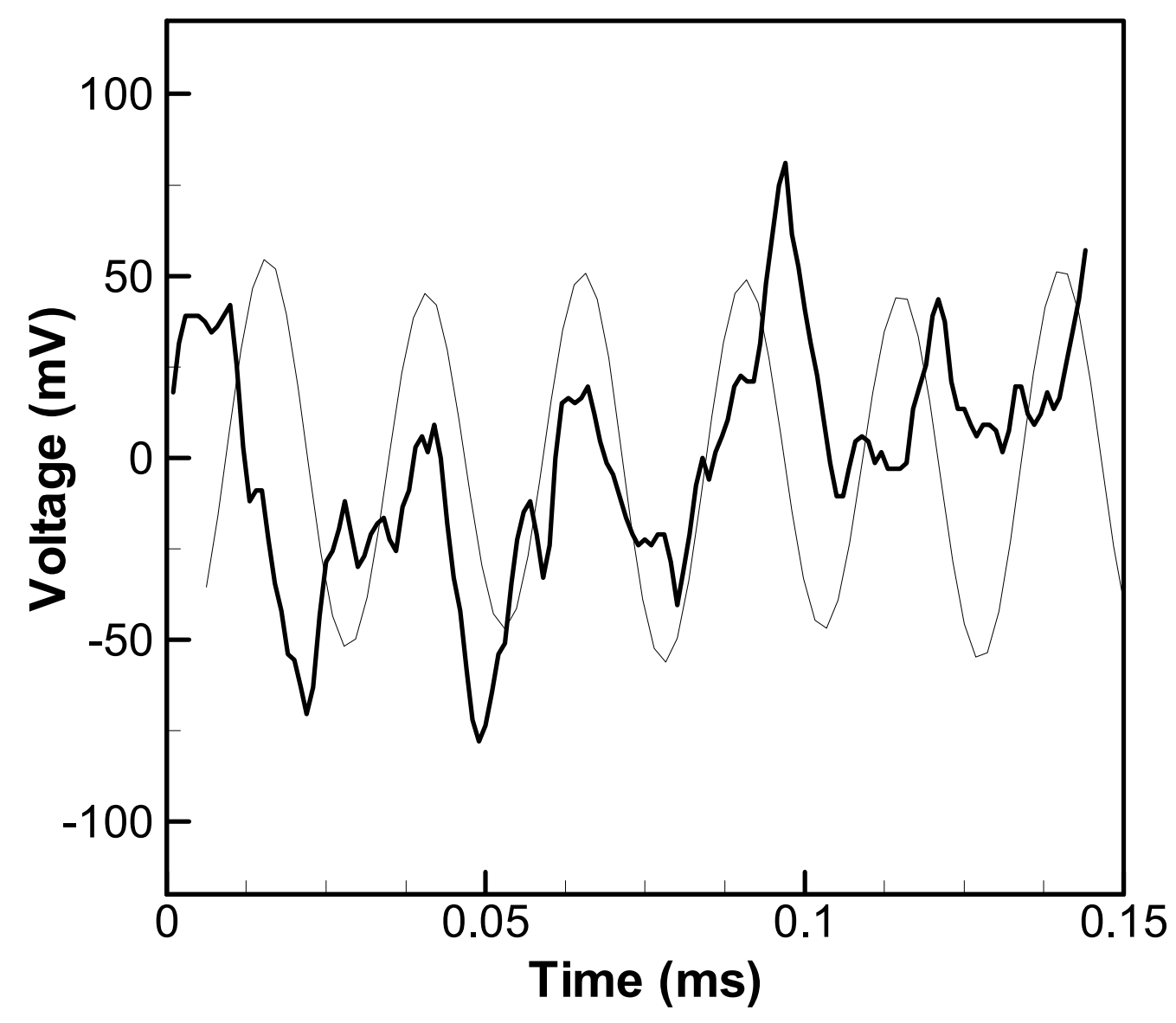

Fig. 39 Multi-harmonic SMIC waves waveform (thick line) in the presence of reduced ion-cyclotron damping factor $(=0.38)$ and single-feature ion cyclotron wave waveform (thin line) when the ion cyclotron damping factor is equal to unity. 

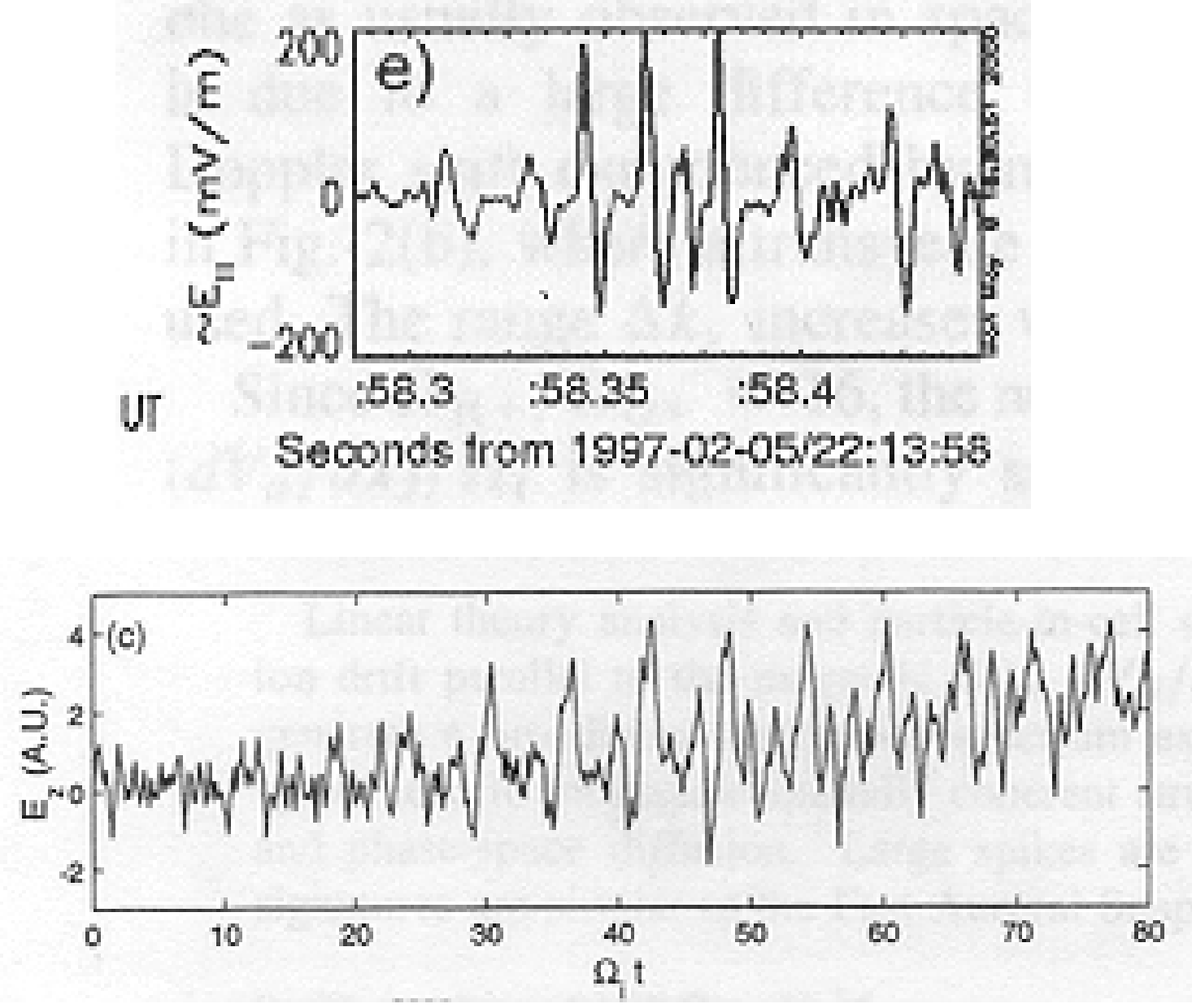

Fig. 40 (a) Space-reported measurements of parallel electric field time-domain fluctuations associated with the presence of multi-harmonic electrostatic ion cyclotron waves [Ergun et al., 1998].

(b) Computer simulations, based on the model of Gavrishchaka et al. [2000], of the parallel electric field time-fluctuations associated with SMIC waves. (reprinted from [Gavrishchaka et al., 2000]). 

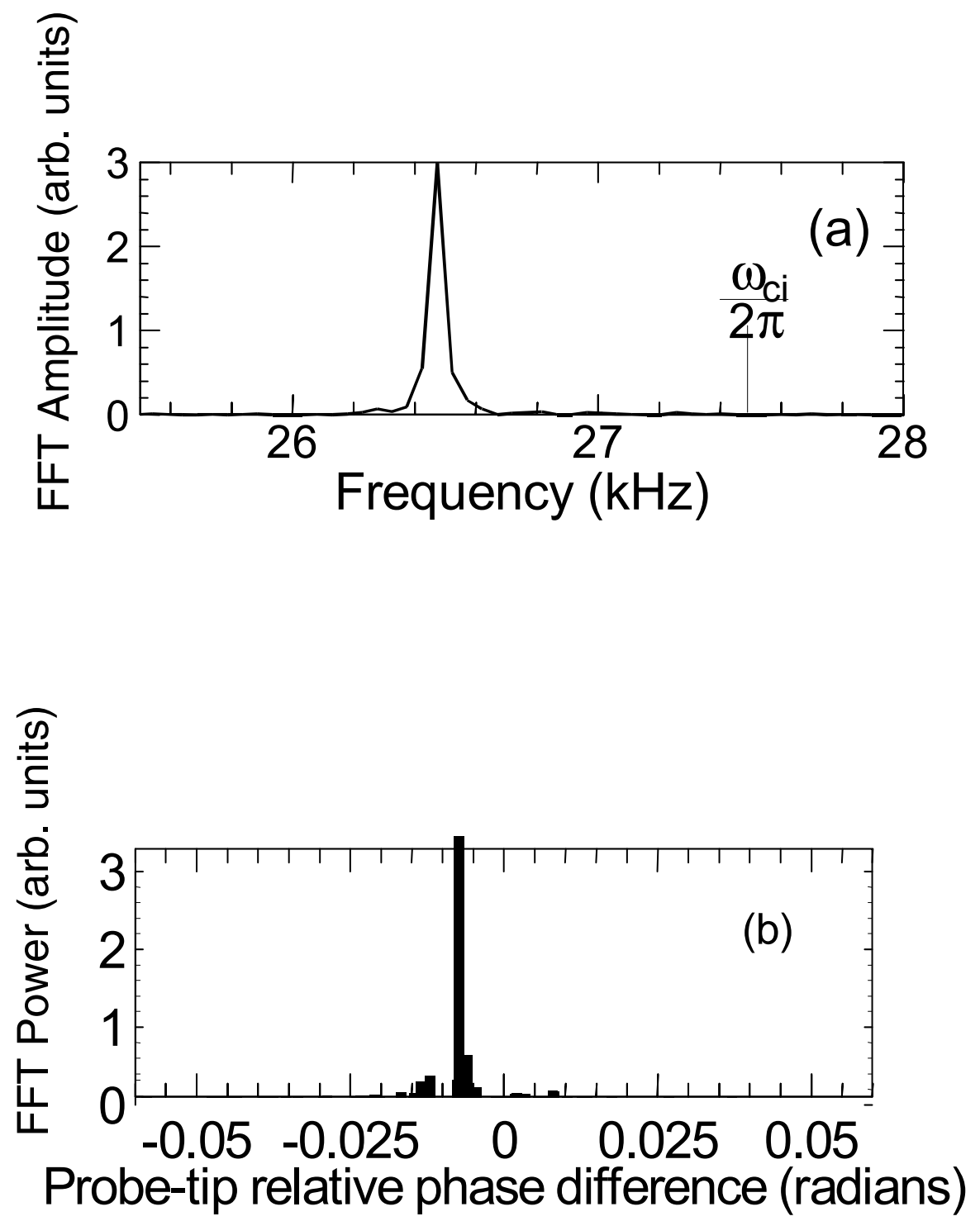

Fig. 41 SMIC waves $k_{z}$ spectrum. The double probe is oriented antiparallel to $B$, thus $k_{z}=-0.026 \mathrm{~cm}^{-1}$. 


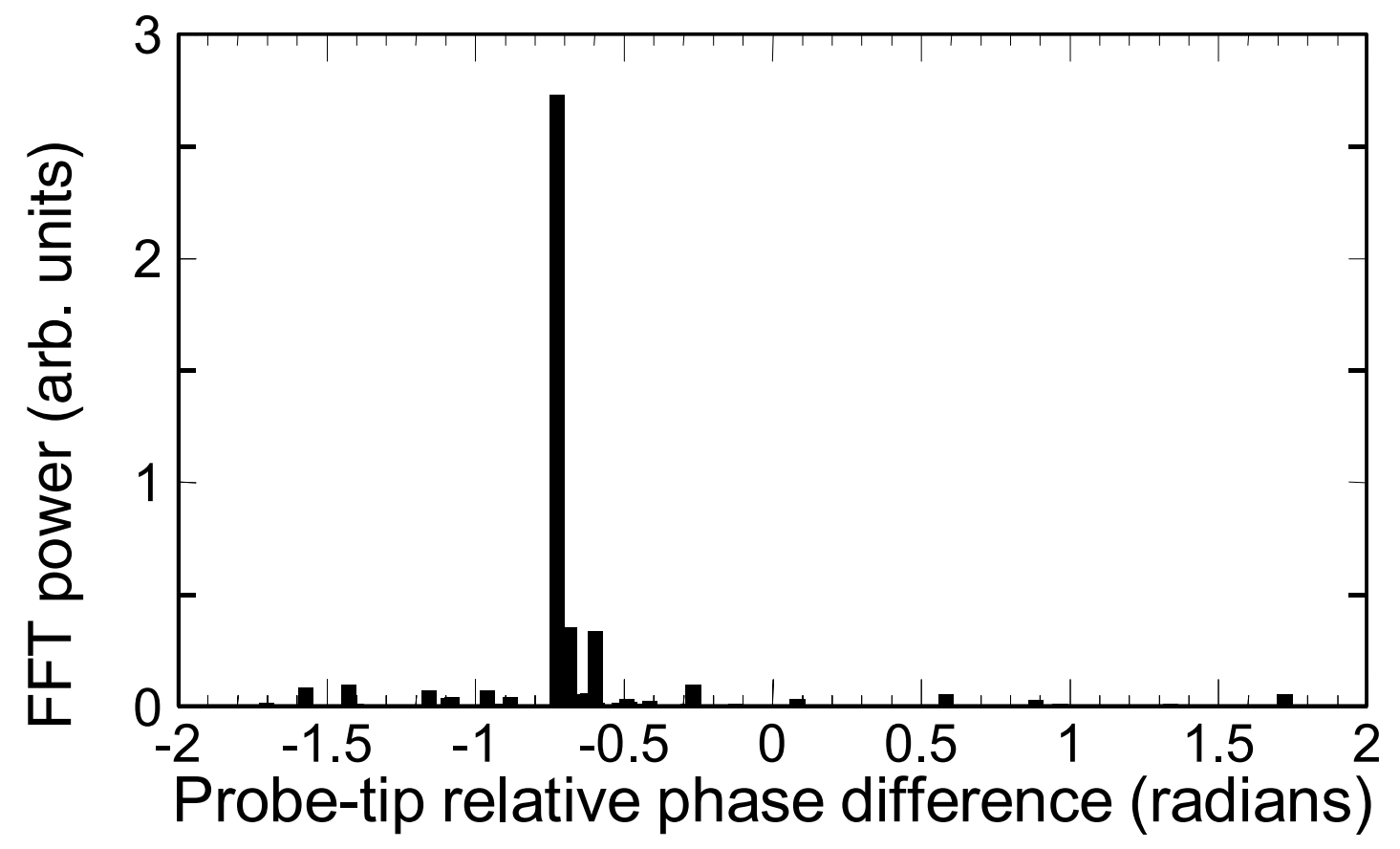

Fig. 42 SMIC waves $k_{y}$ spectrum with the double probe oriented at $90^{0}$ with respect to $B$, thus $k_{y}=-2.21 \mathrm{~cm}^{-1}$ 


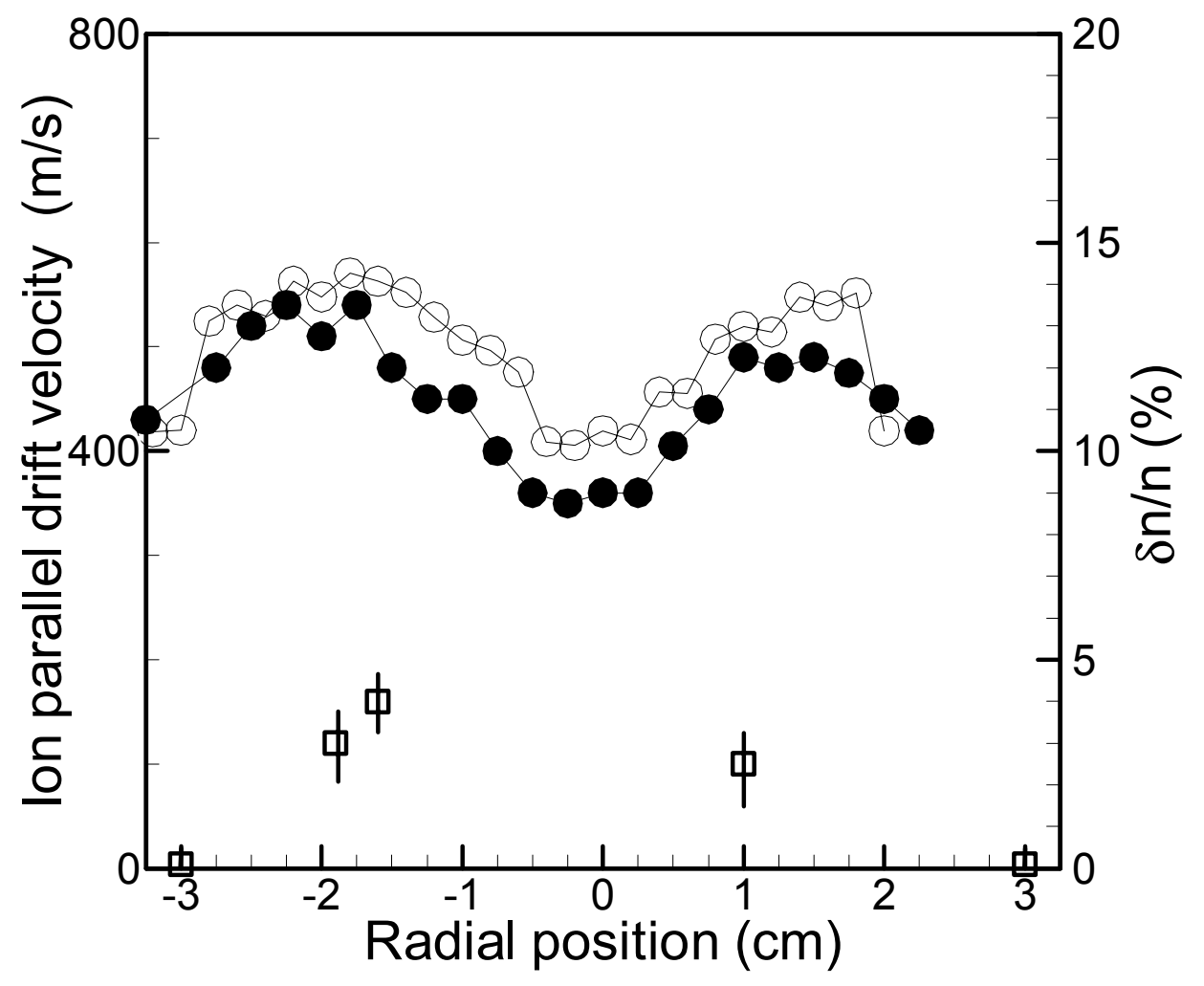

Fig. 43 SMIC waves amplitude radial profile (squares, right scale) peaks within the shear layer.

Measurements are based on the ion saturation current fluctuations recorded by a Langmuir probe. Circles represent the values of the parallel ion drift velocity when using the button electrode (open circles) and the annular electrode (filled circles), respectively. 

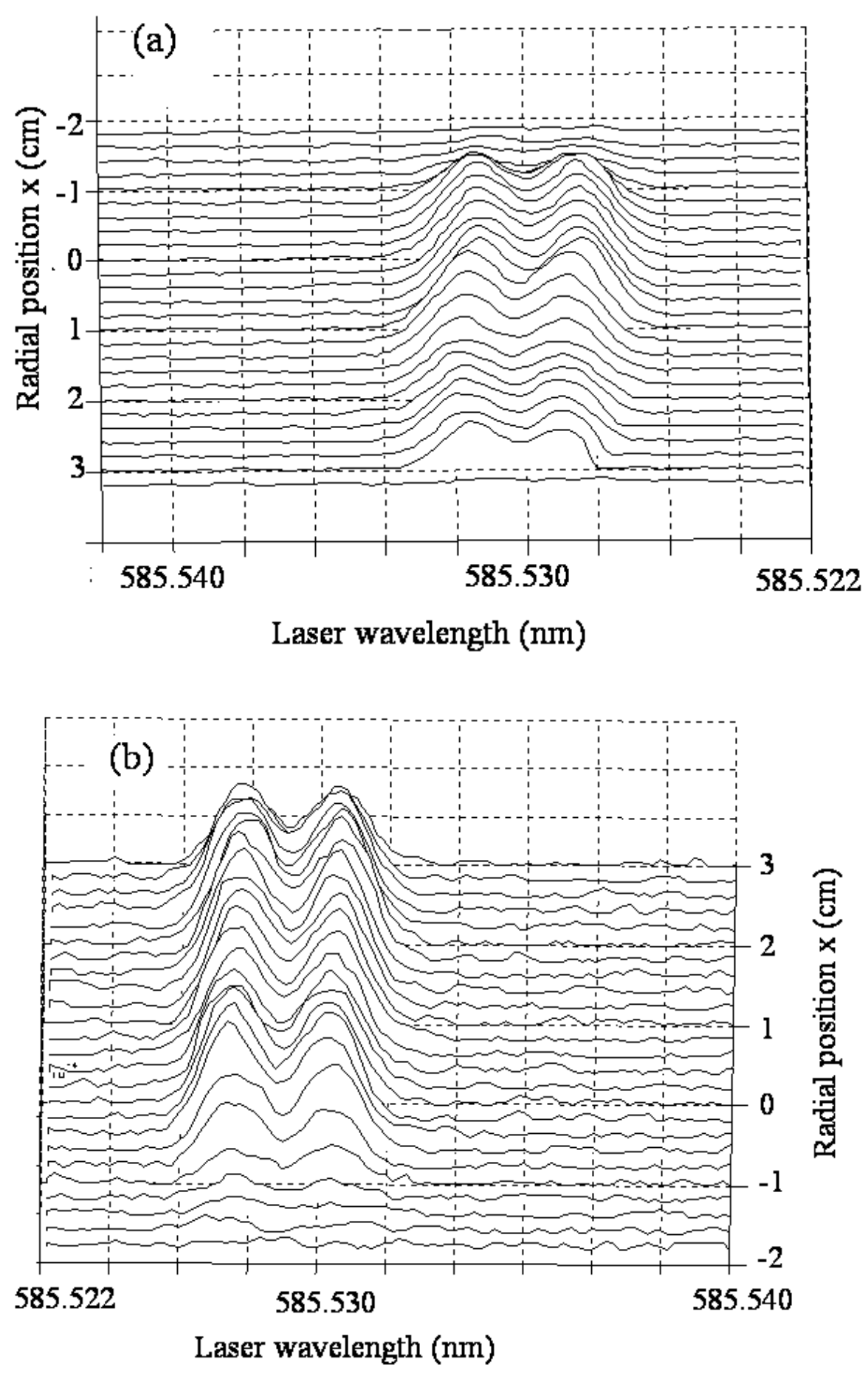

Fig. 44 Radial profile of the parallel-LIF barium spectrum; (a) barium ions produced at both Qmachine ends or (b) only at the north end. Height is proportional to the signal relative intensity. The signal is double-peaked due to the large Zeeman split. The frequency-shift of the signal around $x=0.4 \mathrm{~cm}$ indicates shear in the parallel-ion-velocity drift. Hollow-cathode lamp signal has a wavelength $\lambda_{0}=585.5302 \mathrm{~nm}$. This means small drift for locations between $0.4<x<3 \mathrm{~cm}$ and large drift for locations between $-2<x<0.4 \mathrm{~cm}$. 

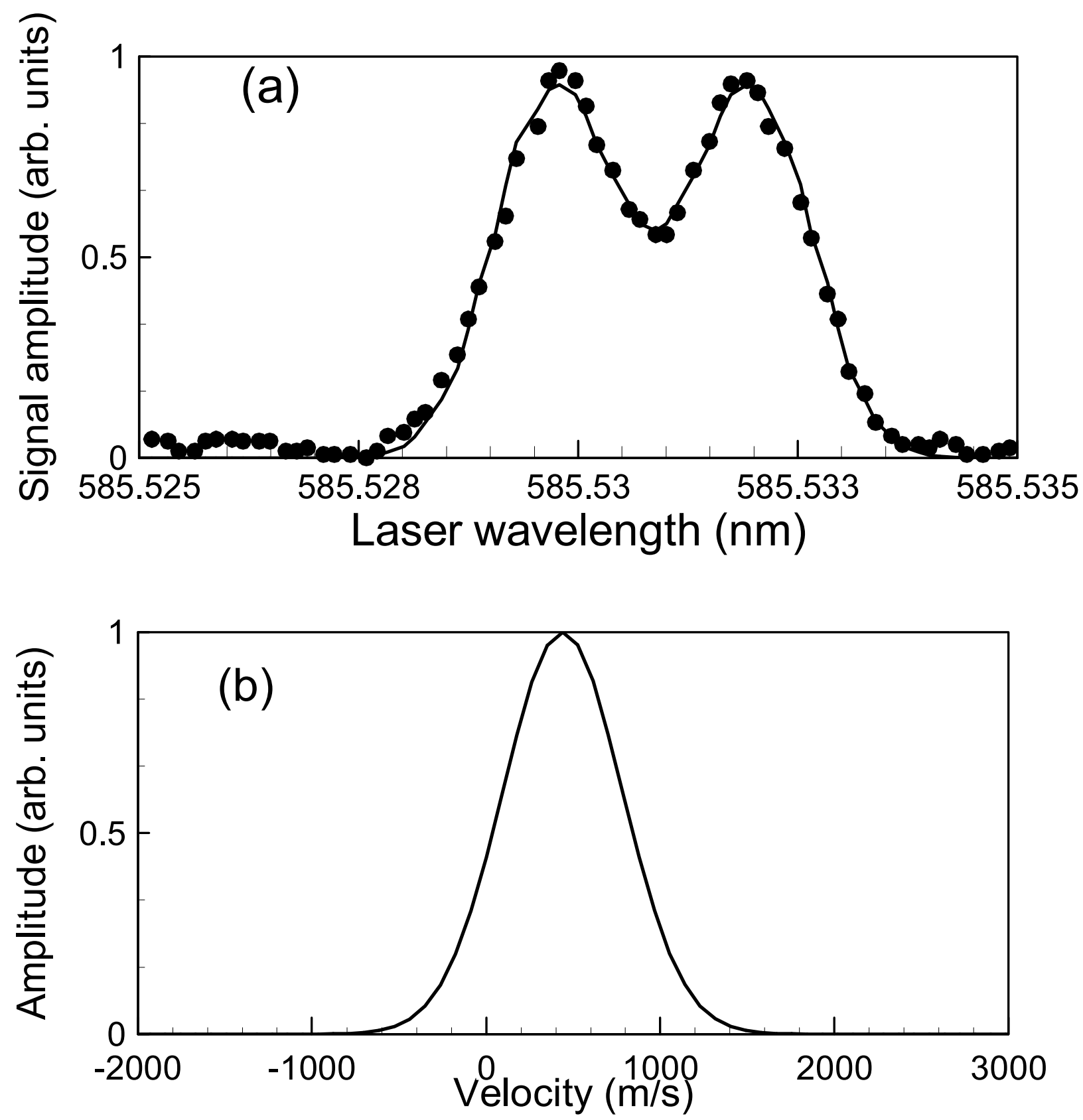

Fig. 45 (a) Sample of parallel LIF fitting and (b) the corresponding best-fit singleMaxwellian ion distribution after the Zeeman-split removal. 


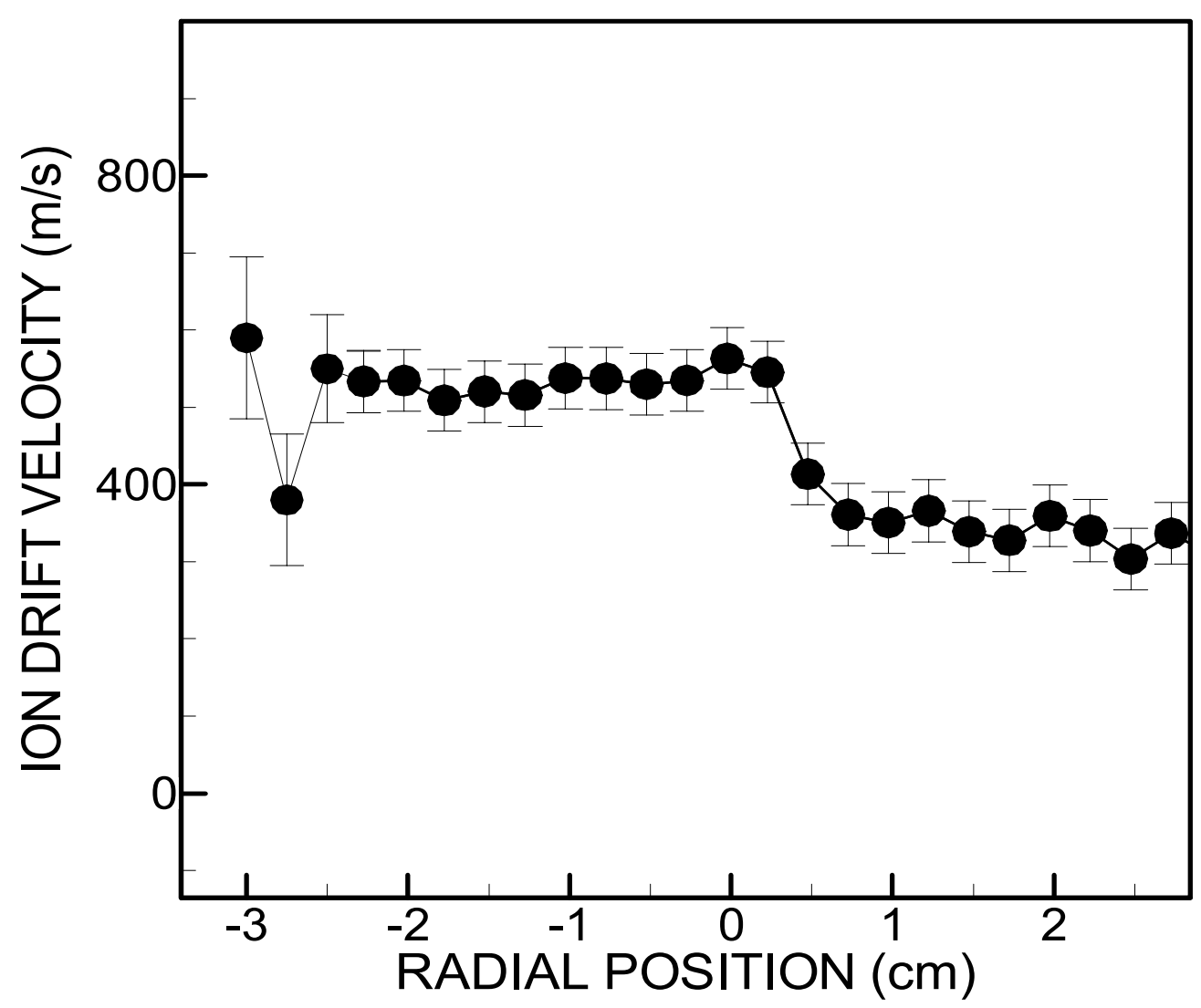

Fig. 46 Typical radial profile of the parallel ion drift velocity when SMIA waves are destabilized. 


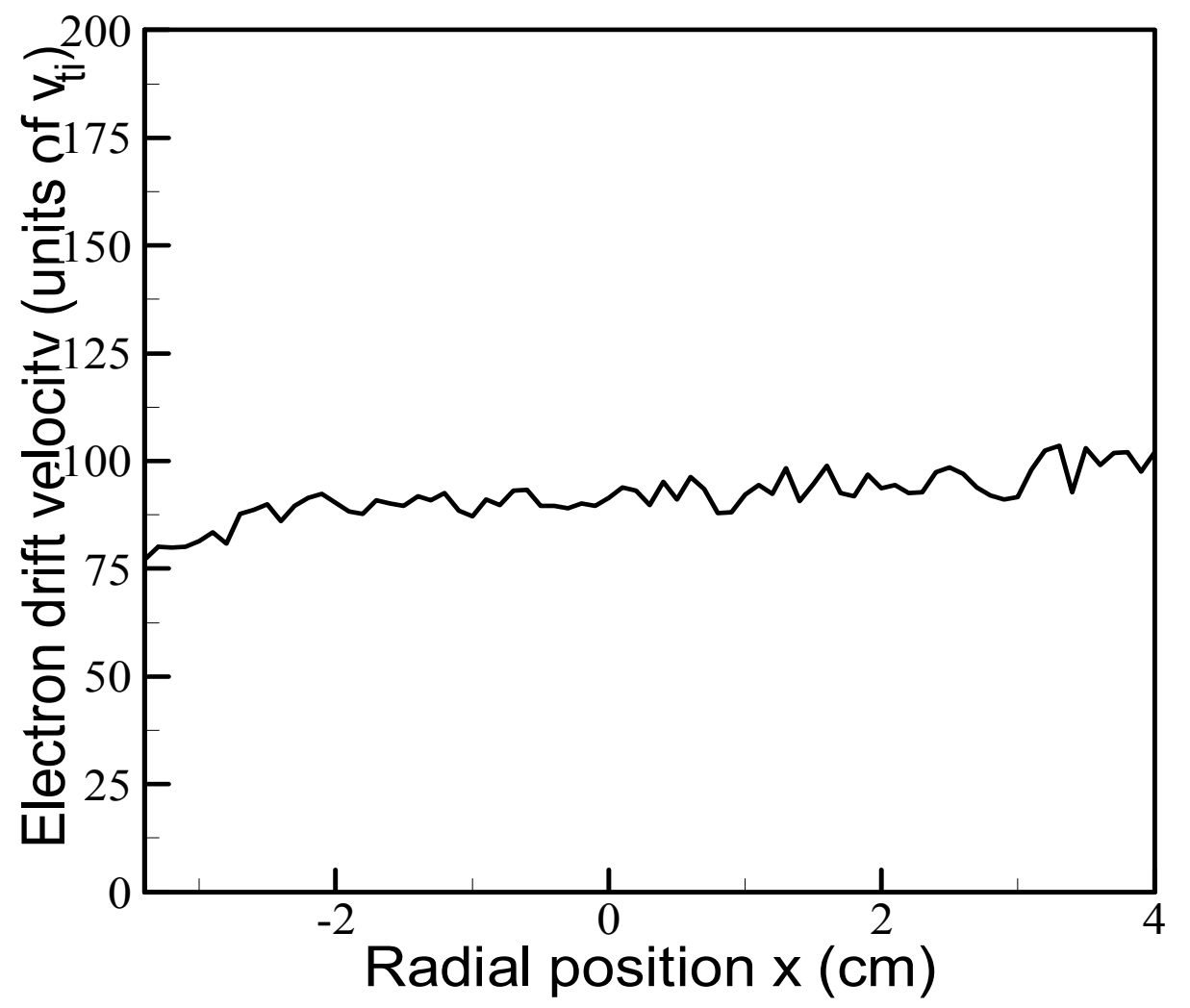

Fig. 47 Parallel electron drift velocity radial profile in the presence of SMIA waves $\left(v_{t i}=480 \mathrm{~m} / \mathrm{s}\right)$. 

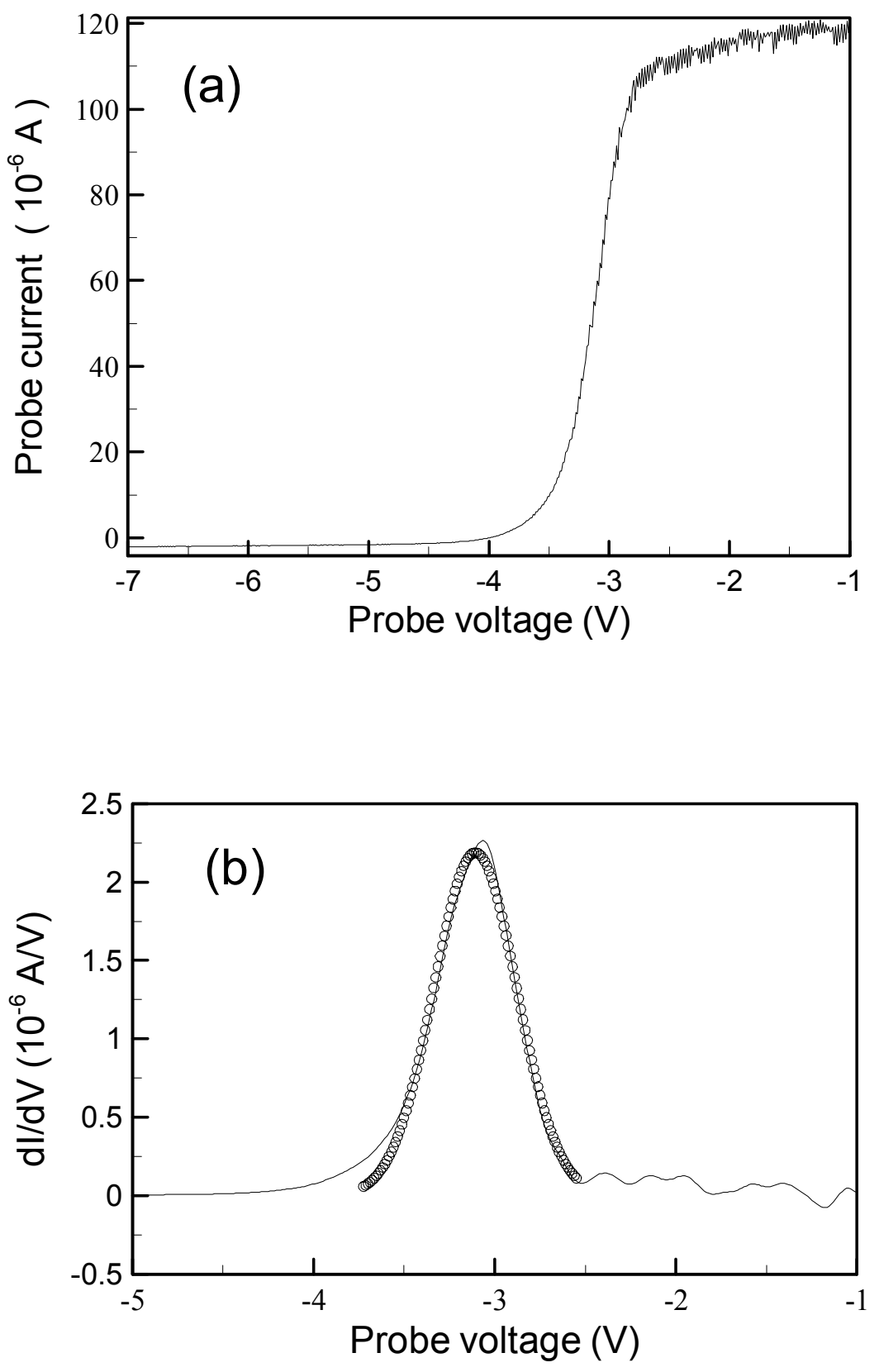

Fig. 48 (a) Plasma current-voltage characteristic in the presence of SMIA waves; (b) current derivative (line) and the best Maxwellian fit (dots). 

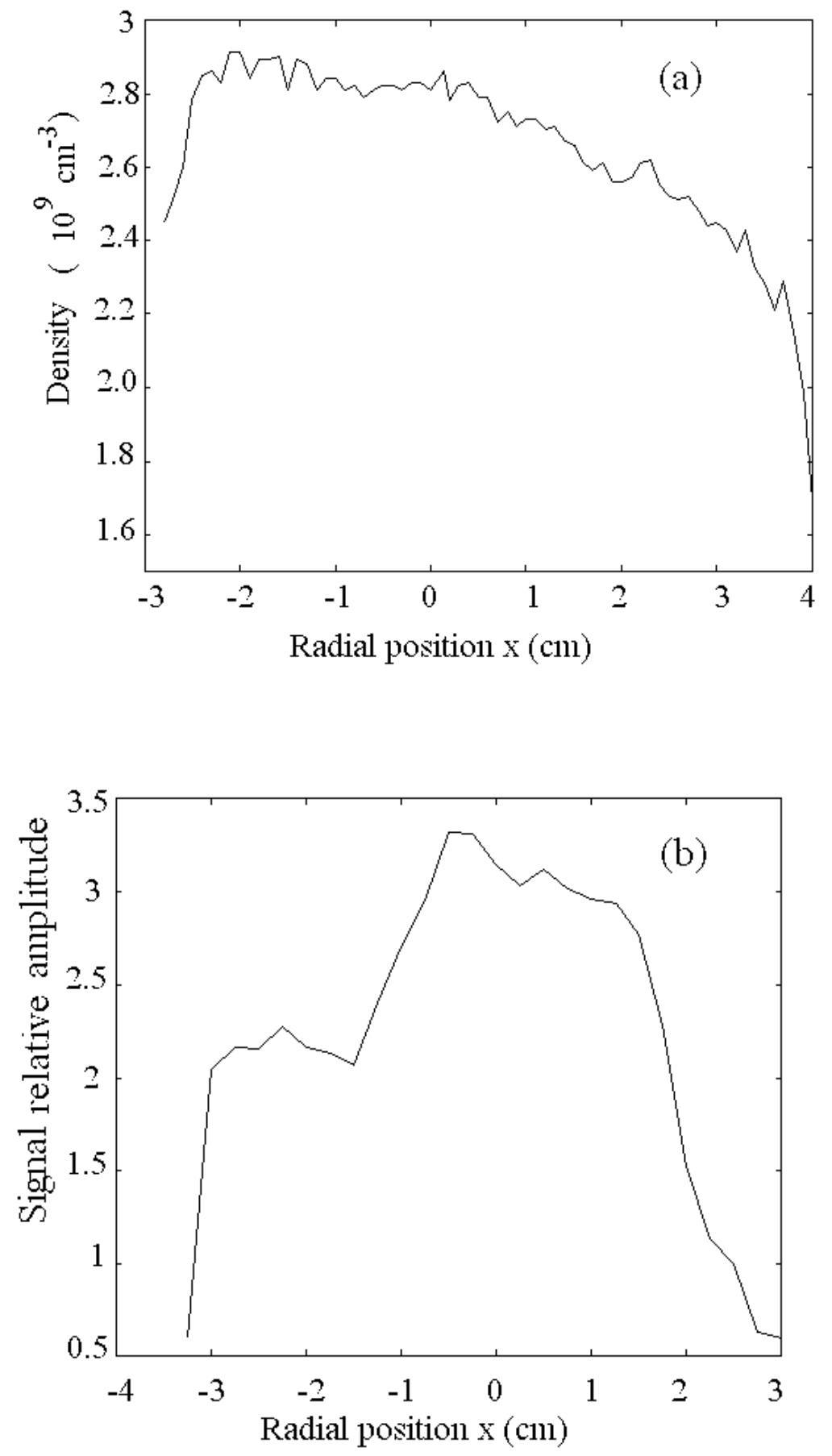

Fig. 49 (a) Plasma density radial profile inferred from ion saturation current measurements; (b) Radial profile of the LIF-signal relative amplitude. 


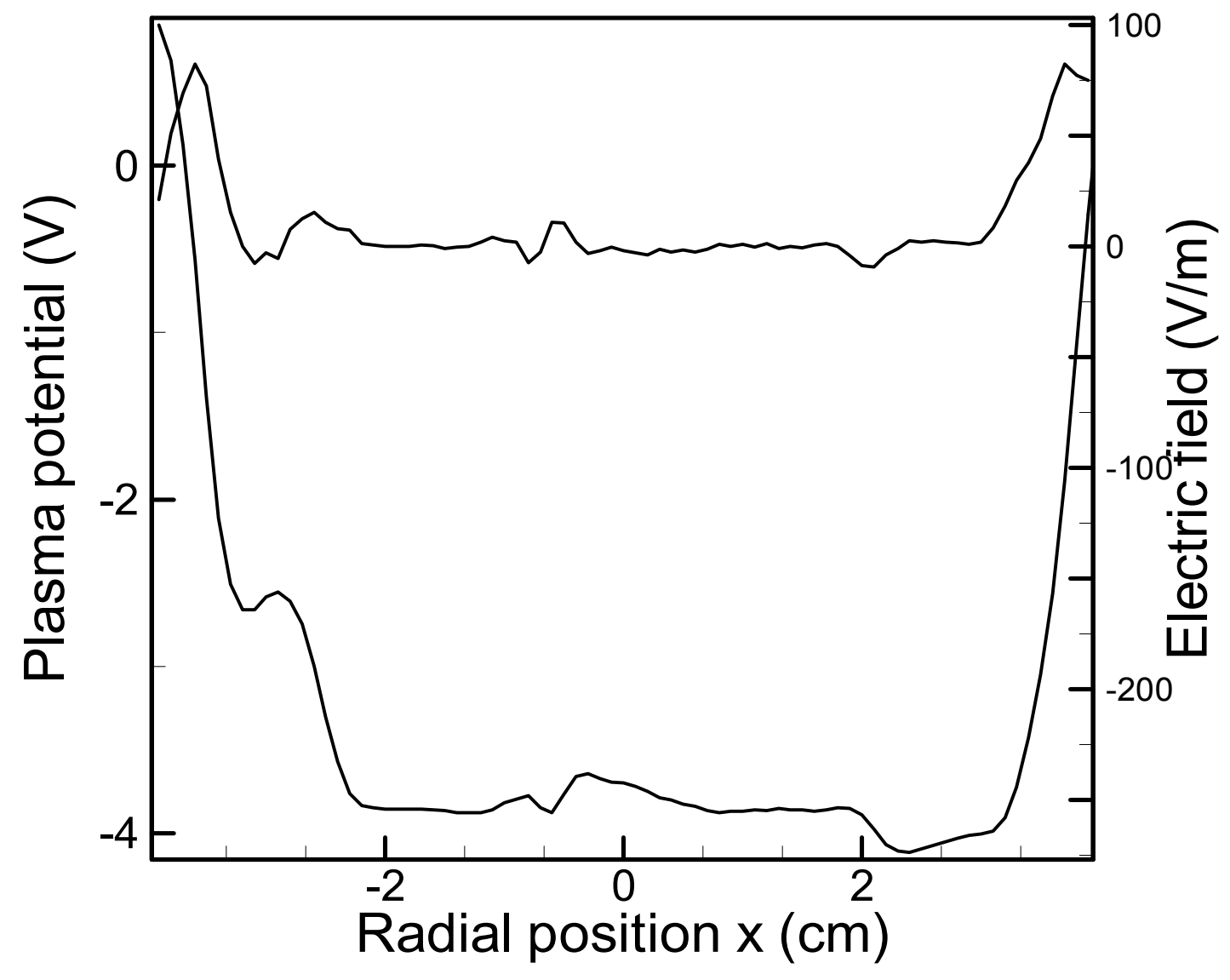

Fig. 50 Plasma-potential radial profile (lower curve, left scale) and the corresponding radial electric field (upper curve, right scale) in the DINE configuration. 


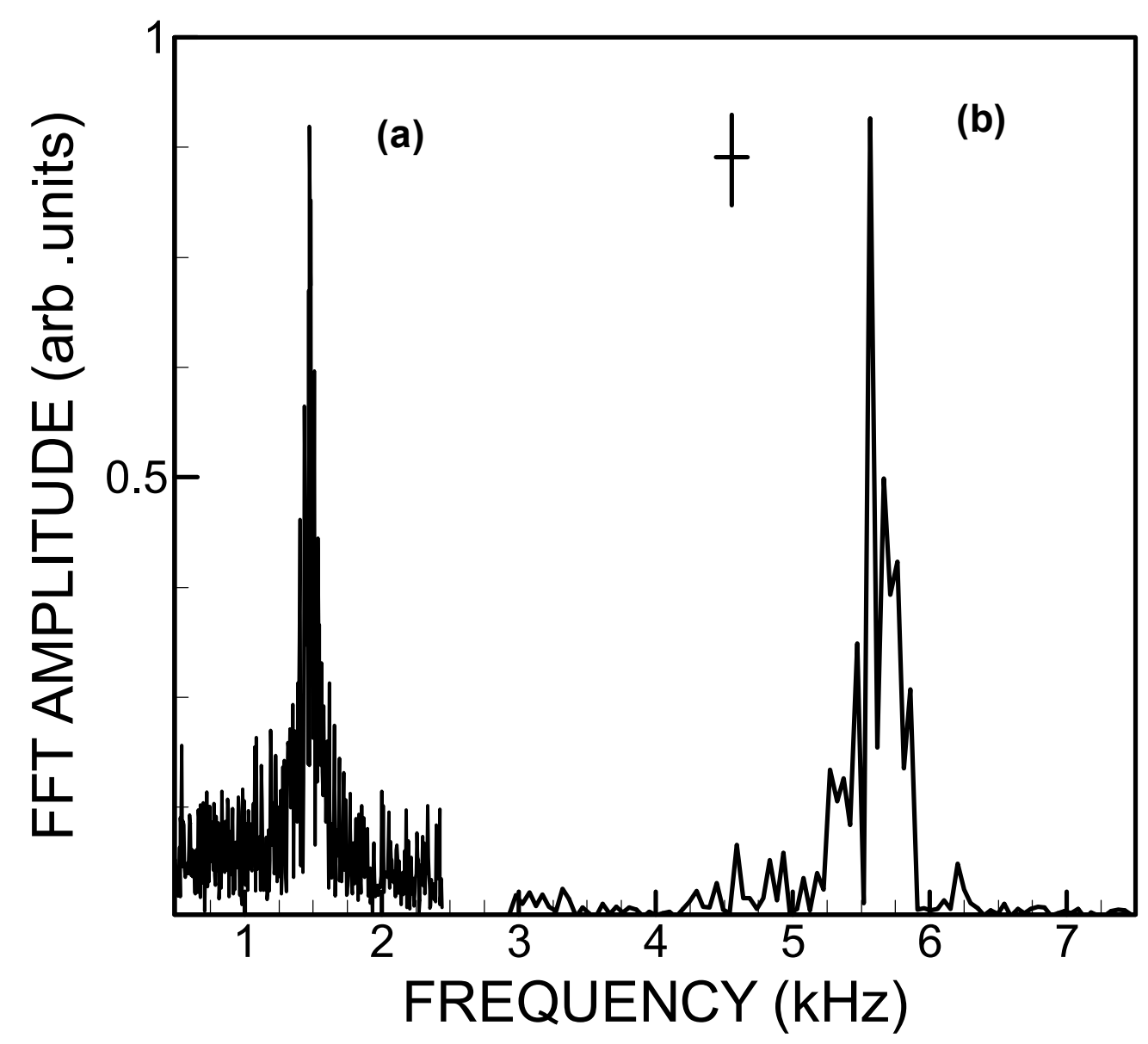

Fig 51 SMIA wave spectrum in the lab frame: (a) wave propagating parallel to the ion flow; (b) wave propagating anti-parallel to the ion flow. Here, $f_{\mathrm{ci}}=13 \mathrm{kHz}$. Doppler shifts are indicated in the text. 


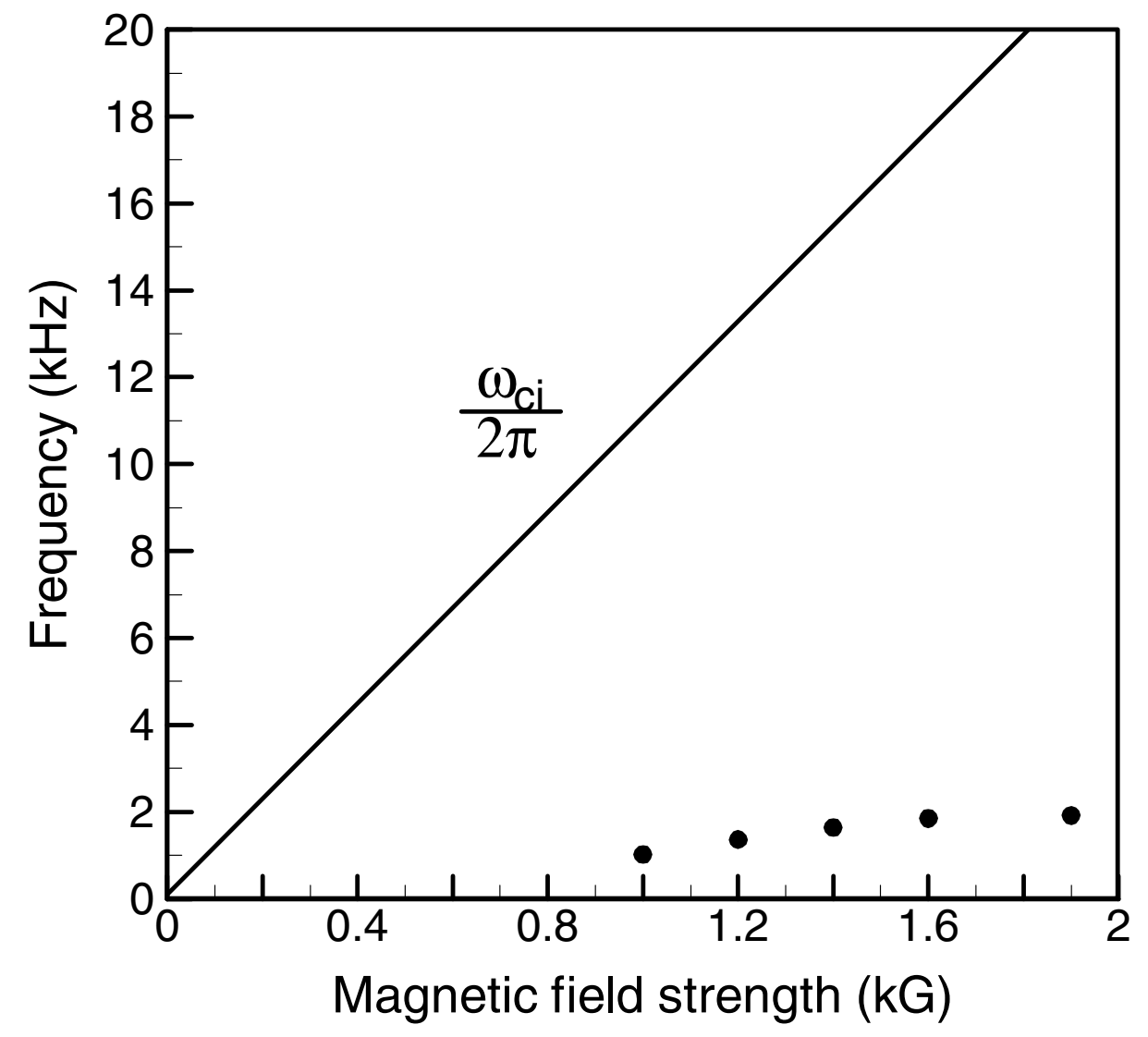

Fig. 52 Dependence of SMIA waves frequency on magnetic field strength. 


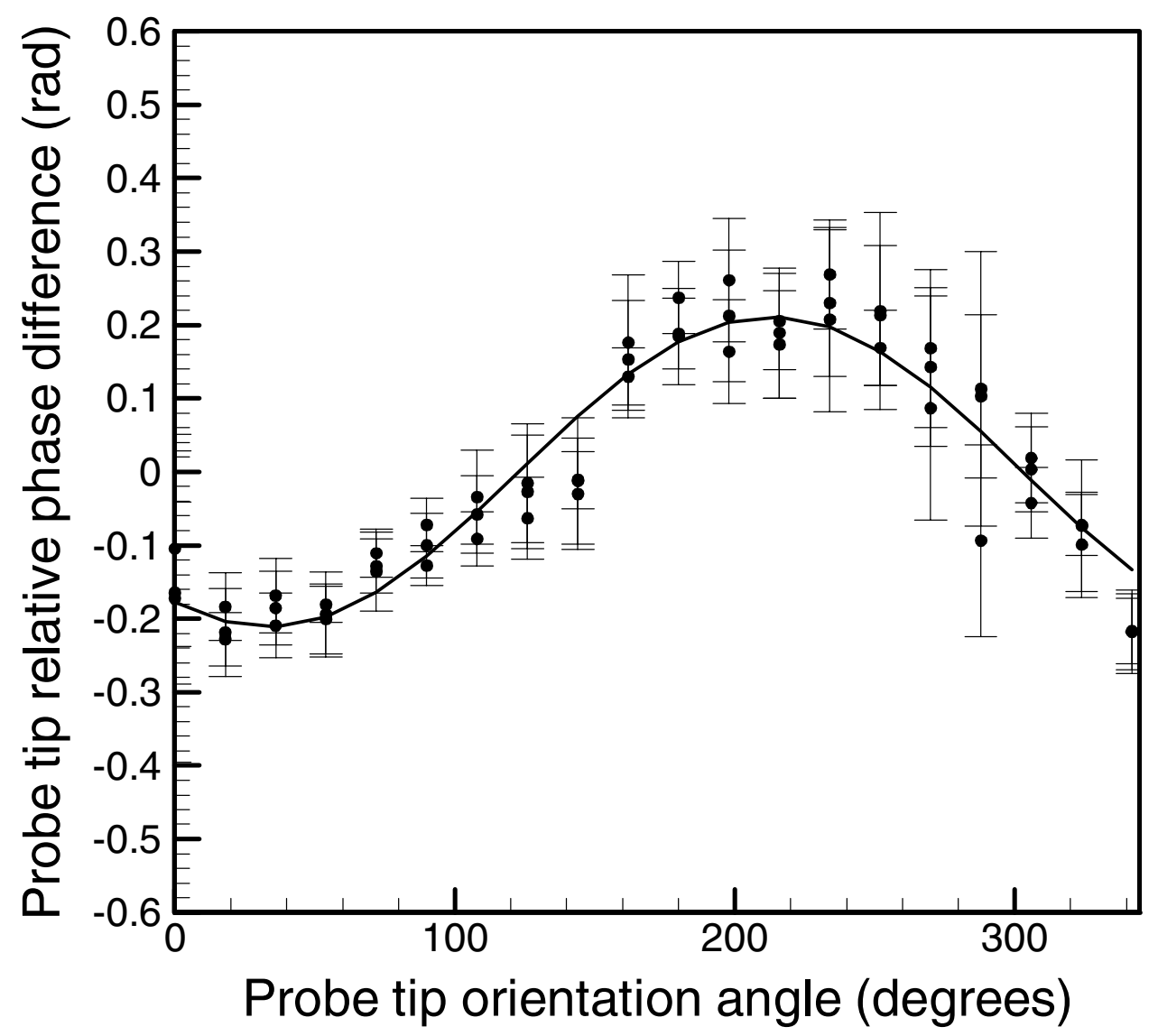

Fig. 53 Probe-tip phase difference as a function of probe orientation angle. The fitted sinusoid yields $k_{z}=-0.507 \mathrm{~cm}^{-1}$ and $k_{y}=-0.331 \mathrm{~cm}^{-1}$. 


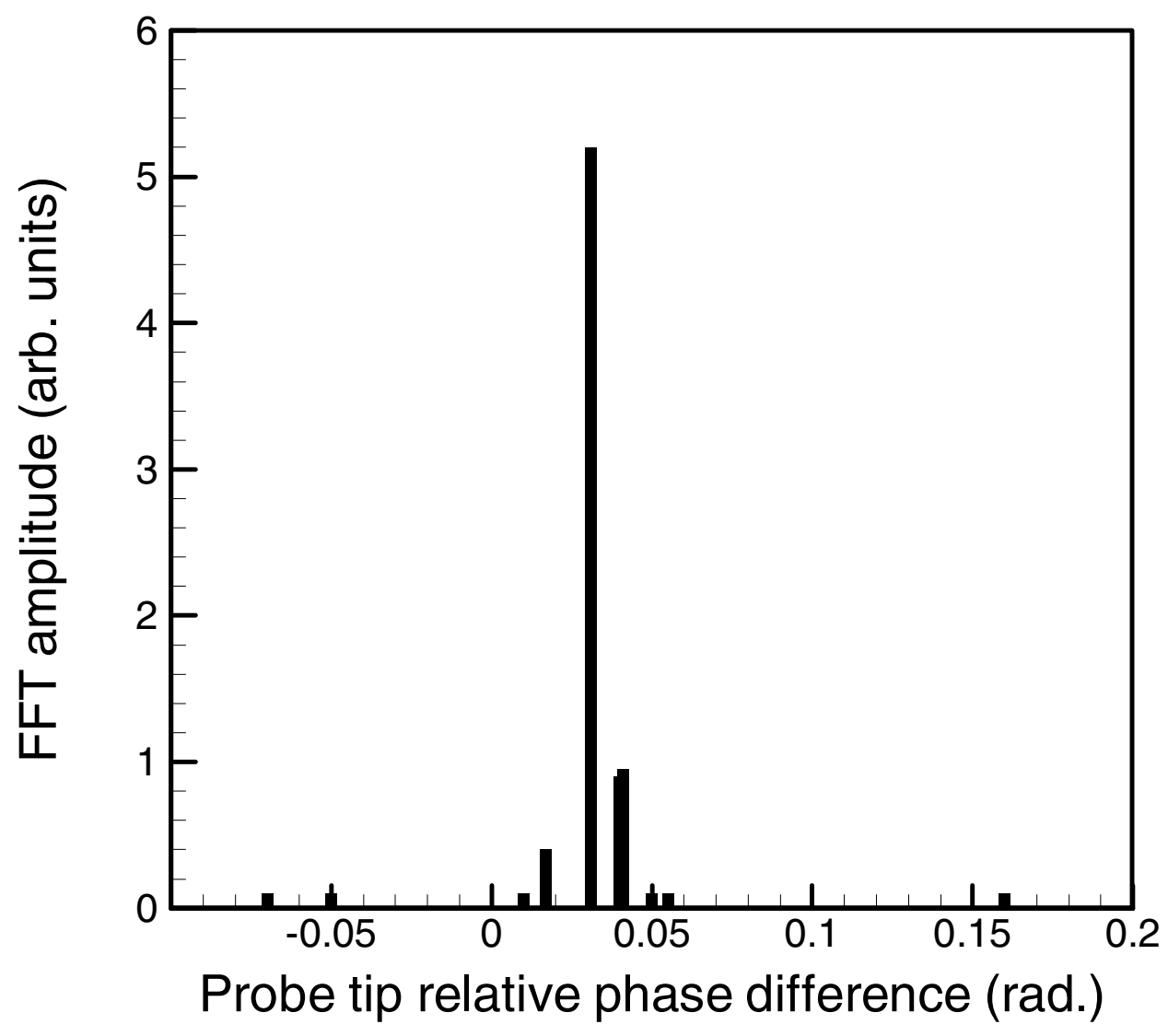

Fig. 54 SMIA waves $k_{y}$ wavevector spectrum with the two-tip probe oriented at $90^{\circ}$ with respect to $B$ lines. $k_{y}=0.09 \pm 0.02 \mathrm{~cm}^{-1}$ corresponds to the maximum amplitude feature. 


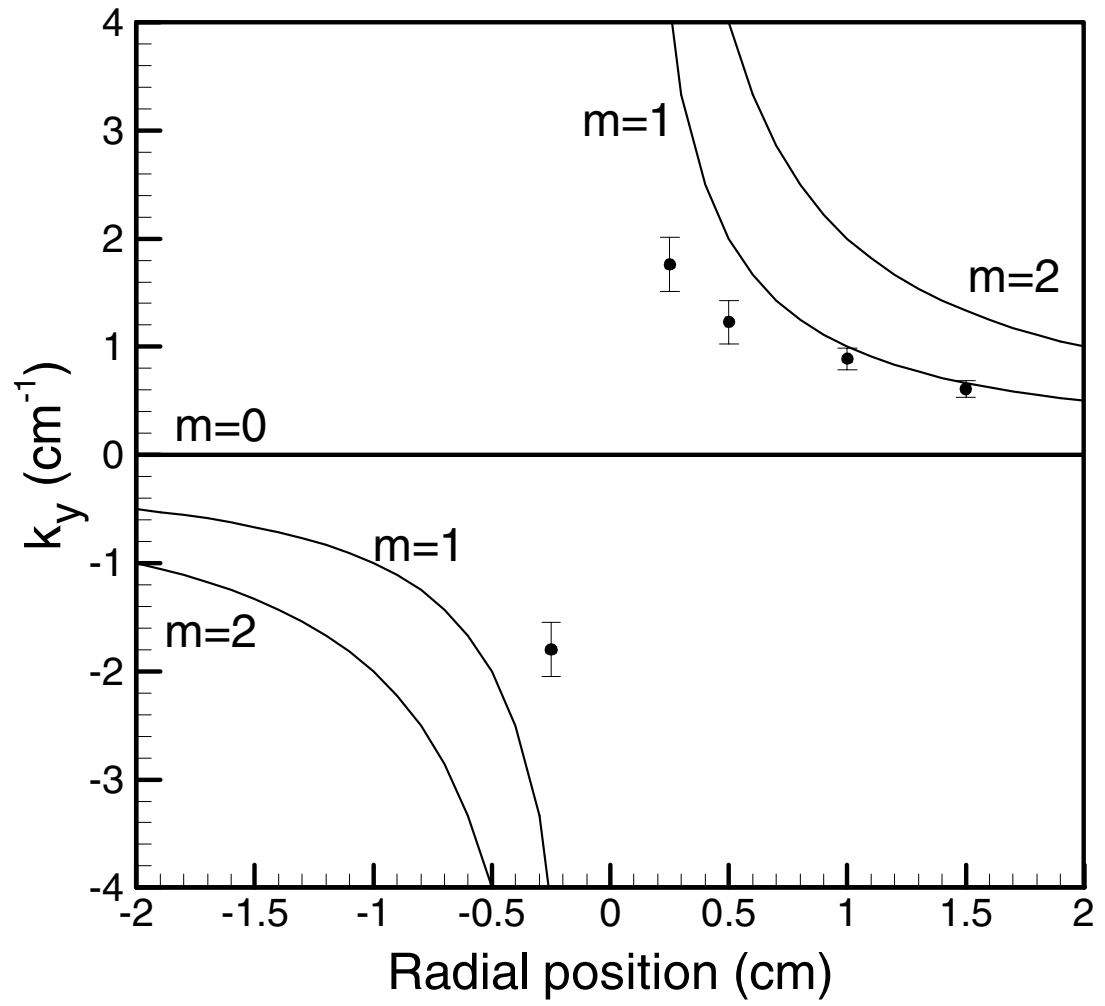

Fig. 55 Comparison between the measured and the predicted SMIA wave azimuthal wavevector for two cases of propagation mode number $m$. 

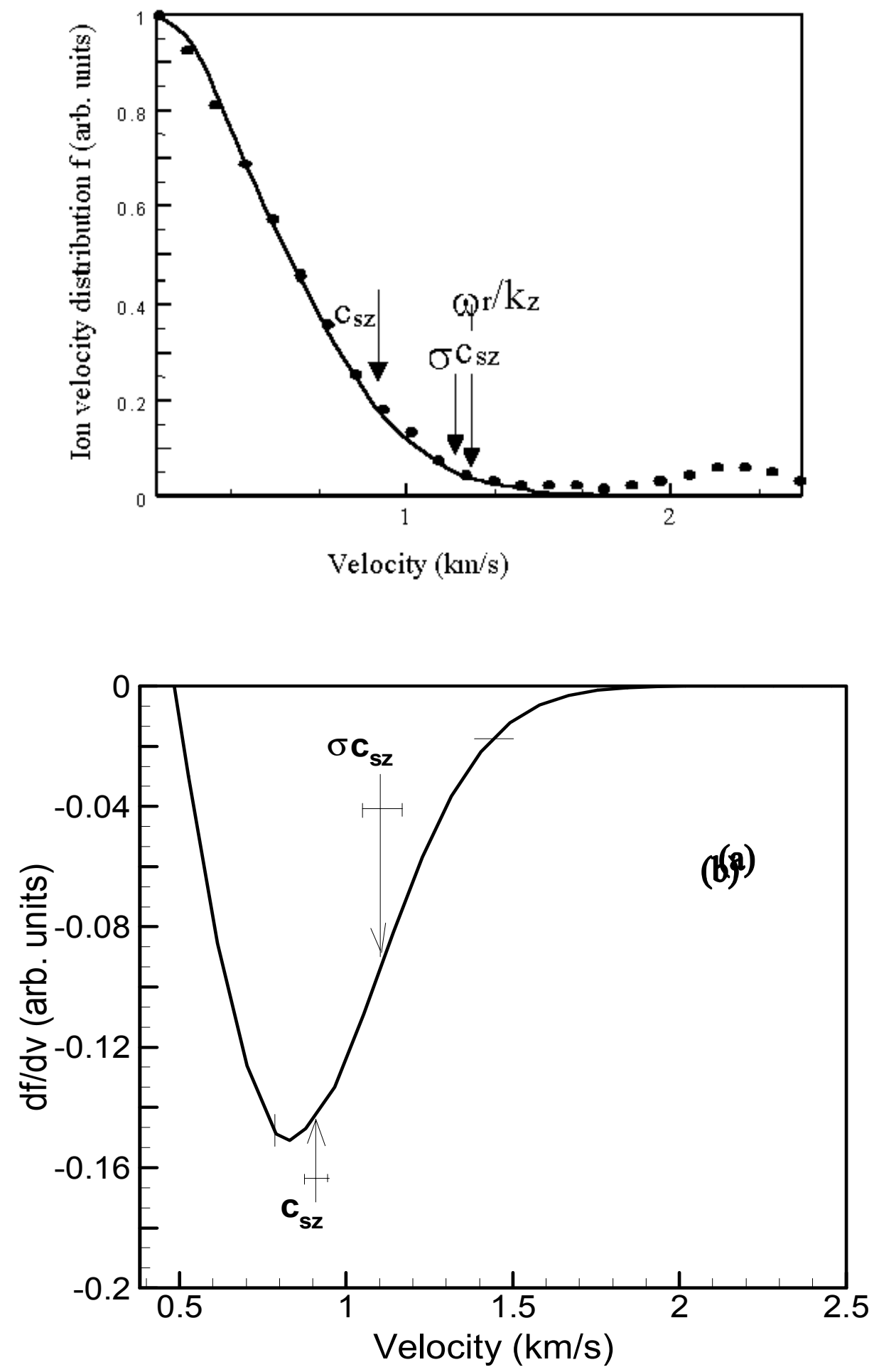

Fig. 56 (a) LIF-measured ion parallel-velocity distribution (circles) and the ion sound speed, measured parallel wave phase velocity and calculated wave phase velocity shift.

(b) Derivative of solid line in Fig.56 (a). The derivative is assumed to be proportional to $\gamma_{\text {iLd. }}$. 


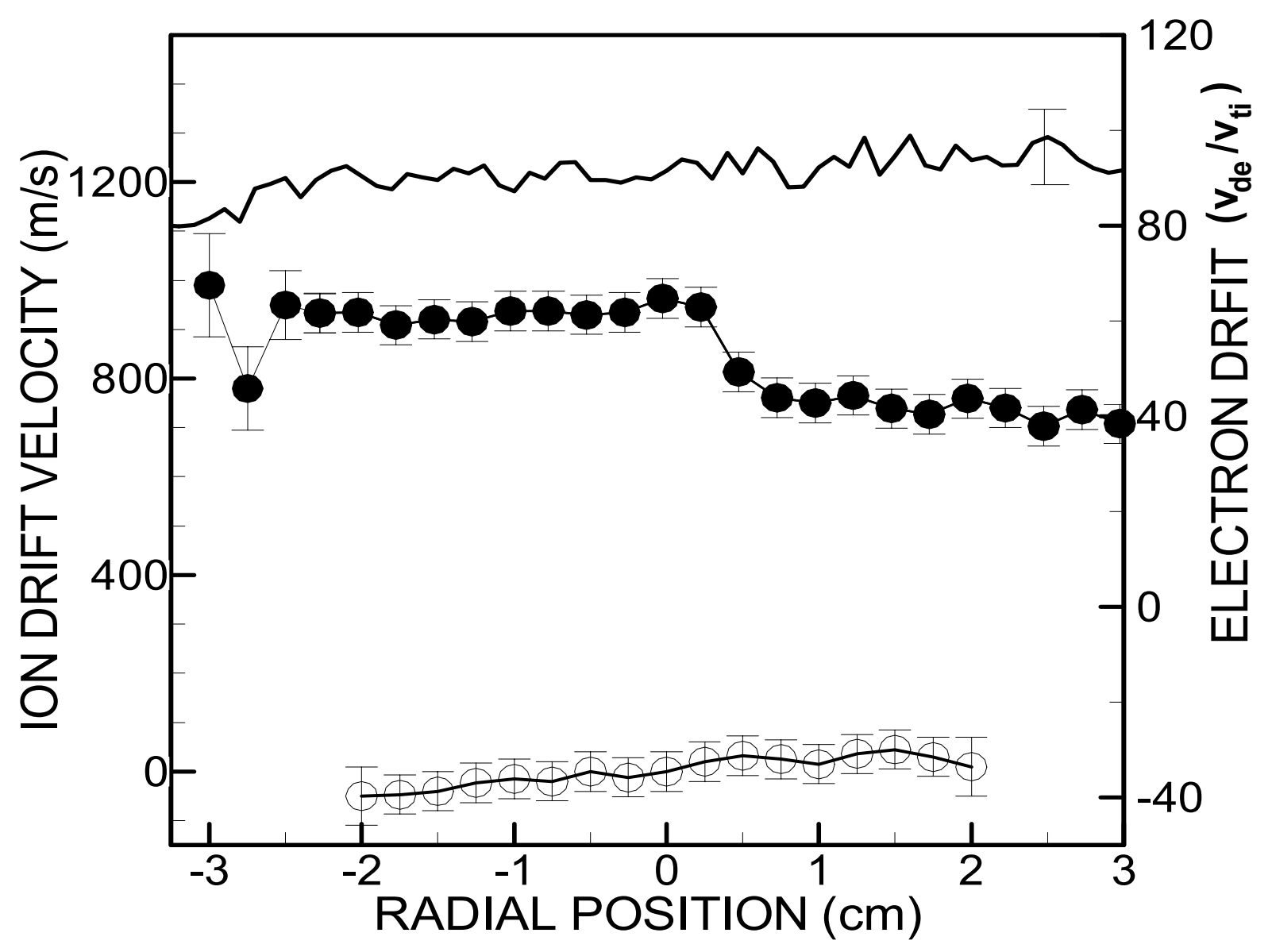

Fig. 57 Comparison between parallel (filled circles), perpendicular (open circles) ion drift velocity profile (left scale), and parallel electron drift velocity (line, right scale) in the presence of SMIA waves. 


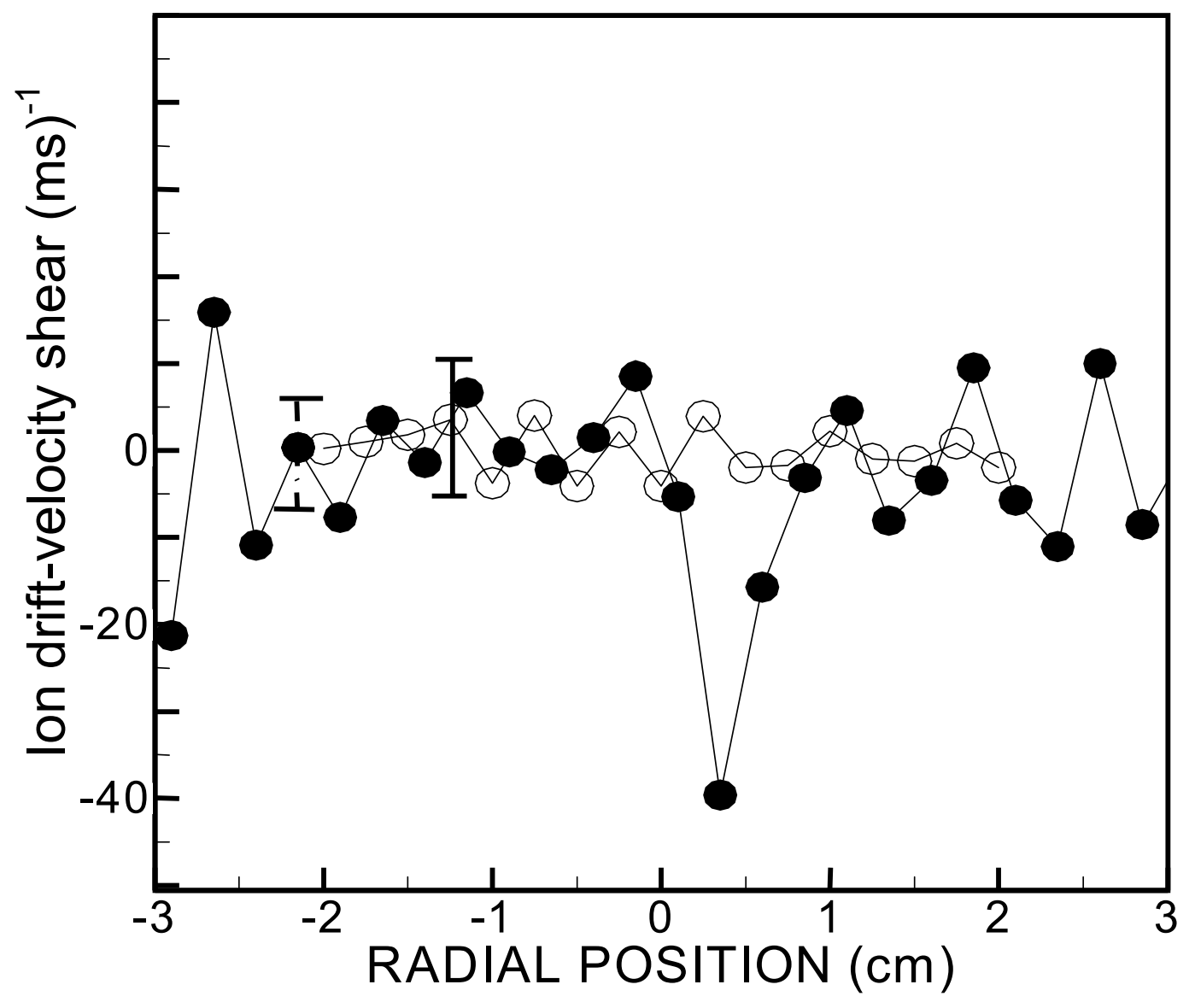

Fig. 58 Comparison between parallel (filled circles) and perpendicular (open circles) ion drift velocity shear in the presence of SMIA waves, based on the profiles shown in Fig. 57. Measurements are taken at $z=46 \mathrm{~cm}$ from the north ionizer (magnetic field $B=0.13 \mathrm{~T}$ is southward). 


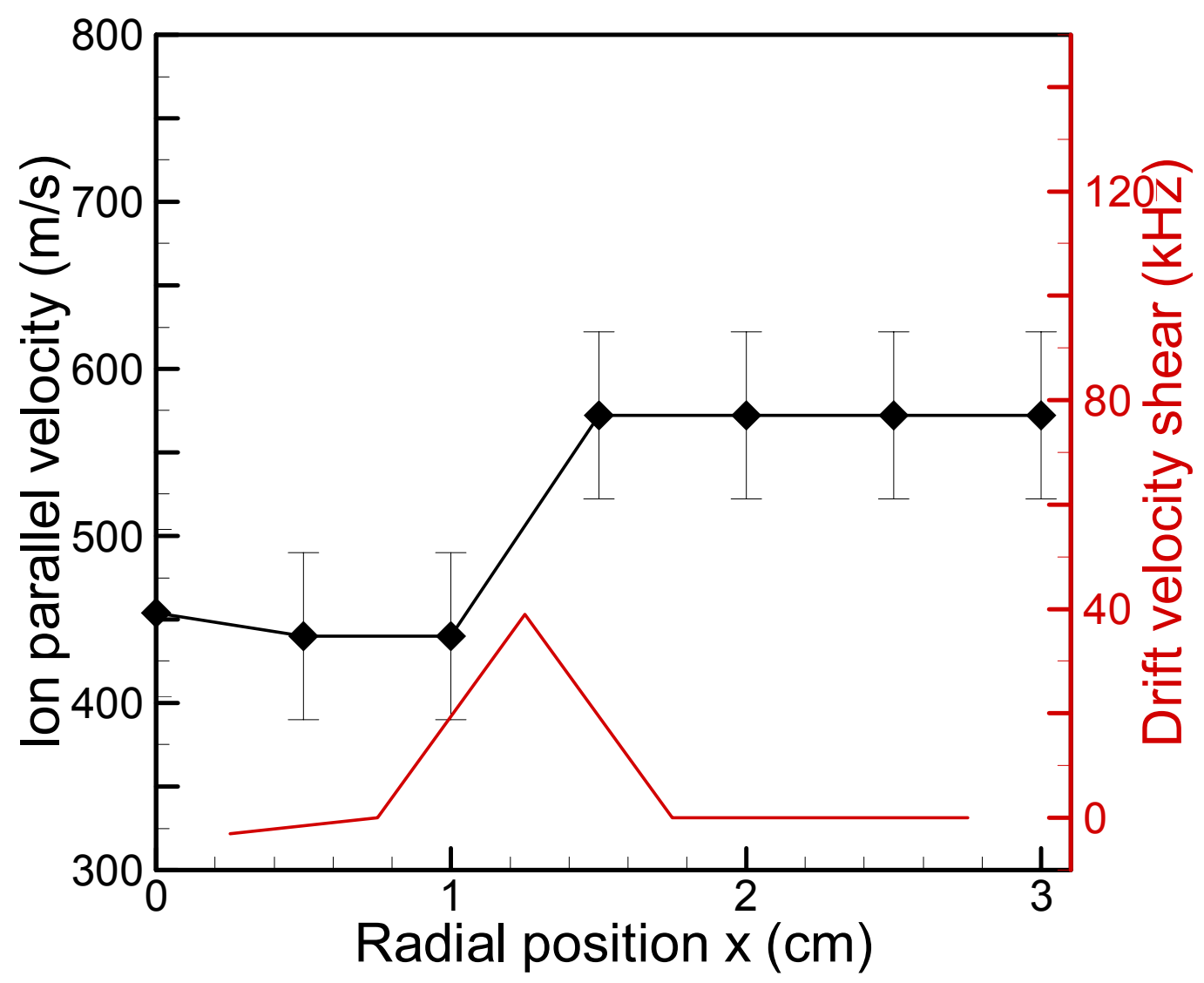

Fig. 59 Parallel ion velocity drift radial profile (upper curve, left scale) and the corresponding positive shear (lower curve, right scale) when no waves are destabilized. Here, $V_{N}=0, V_{S}=0, B=0.13 \mathrm{~T}$ (southward). 


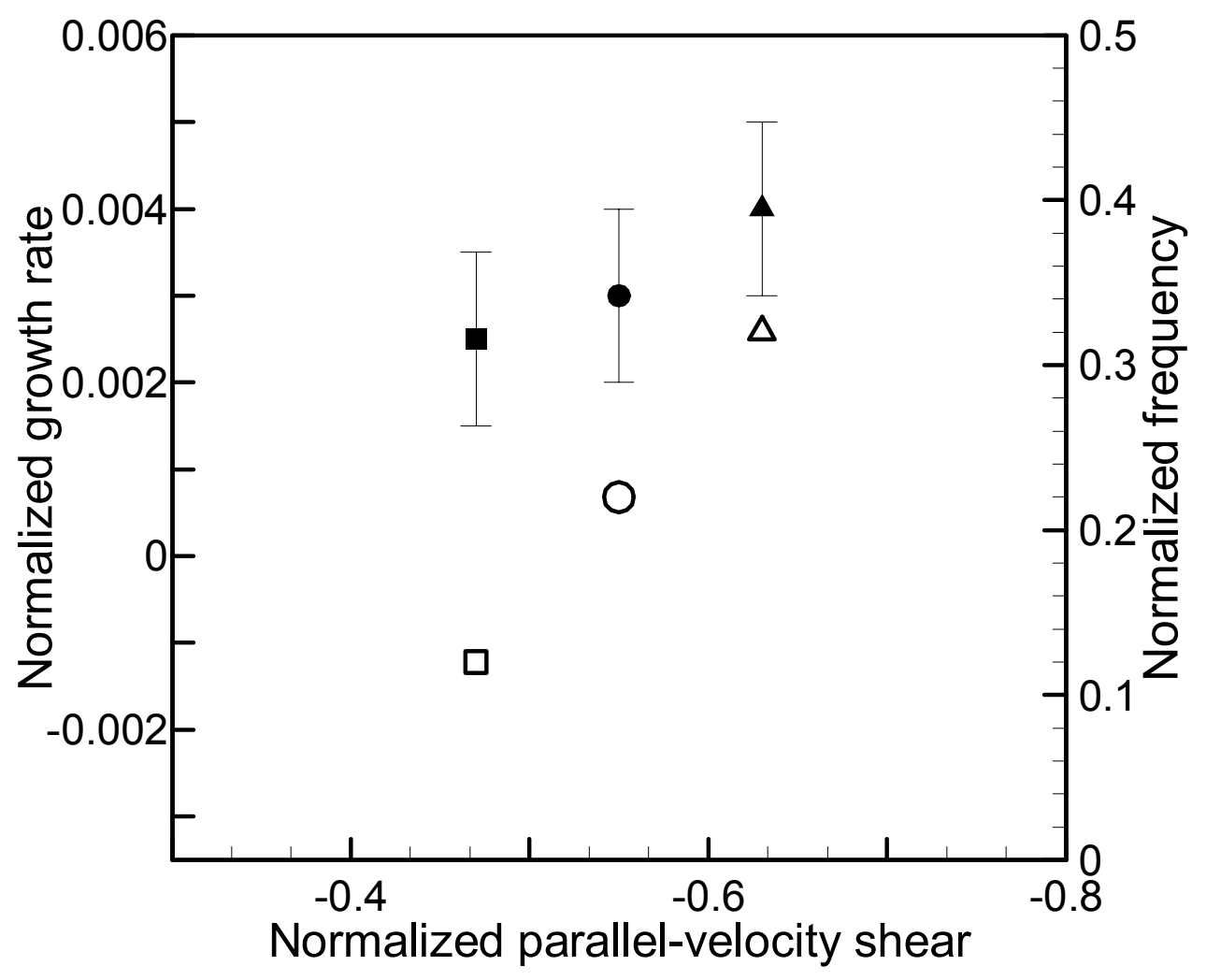

Fig. 60 Correlation between shear, SMIA waves growth rate (filled symbols, left scale) and SMIA waves frequency (open symbols, right scale). Symbols are associated with the values of $u=0.7$ (square), 1.4 (circle) and 1.9 (triangle), respectively. Normalization constant is the ion cyclotron angular frequency $\omega_{\mathrm{ci}}$. 

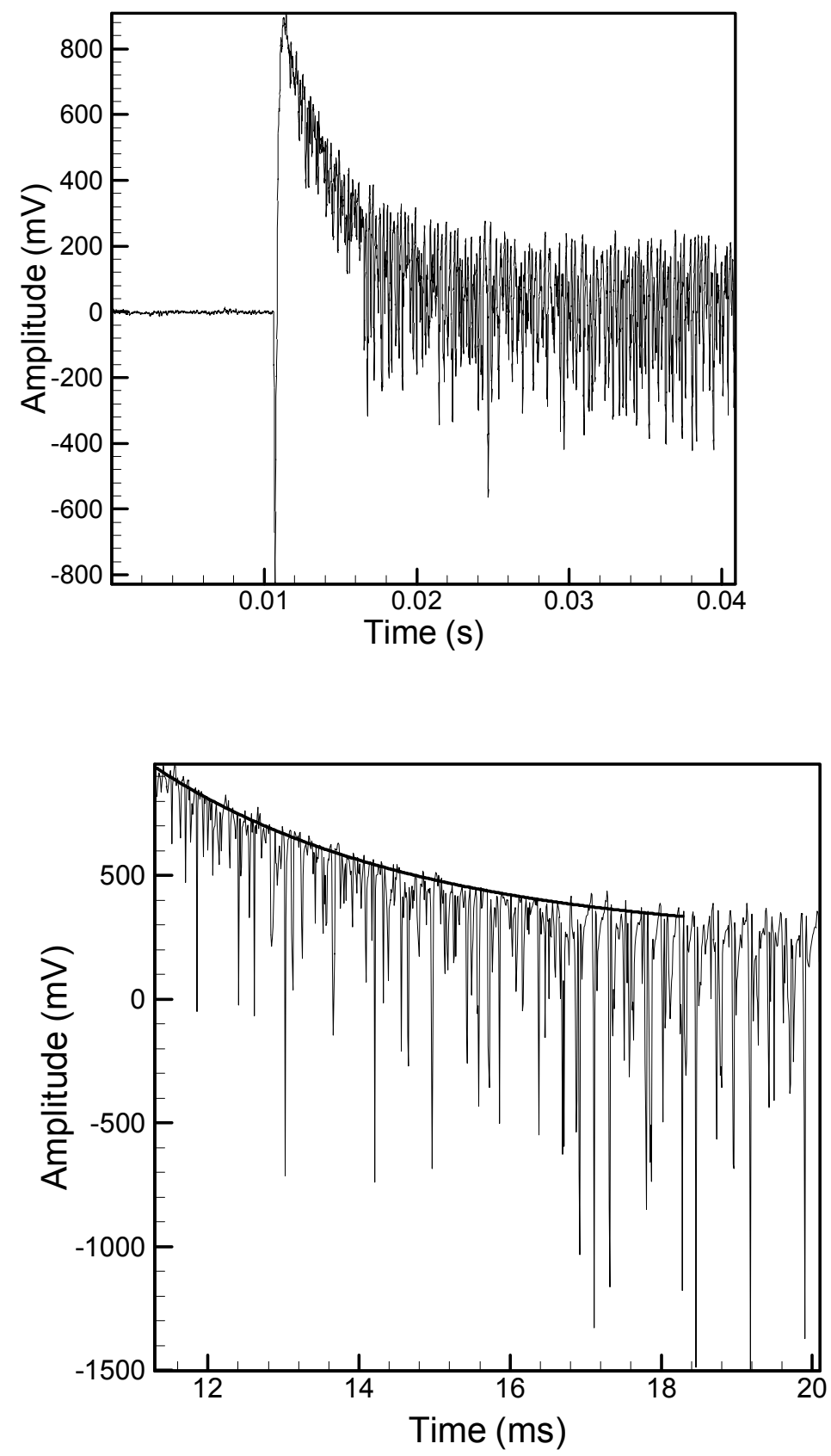

Fig. 61 (a) Wave amplitude temporal growth after applying the stepped ionizer bias (up). Wave amplitude and the pulse-removing fitting line (down). 

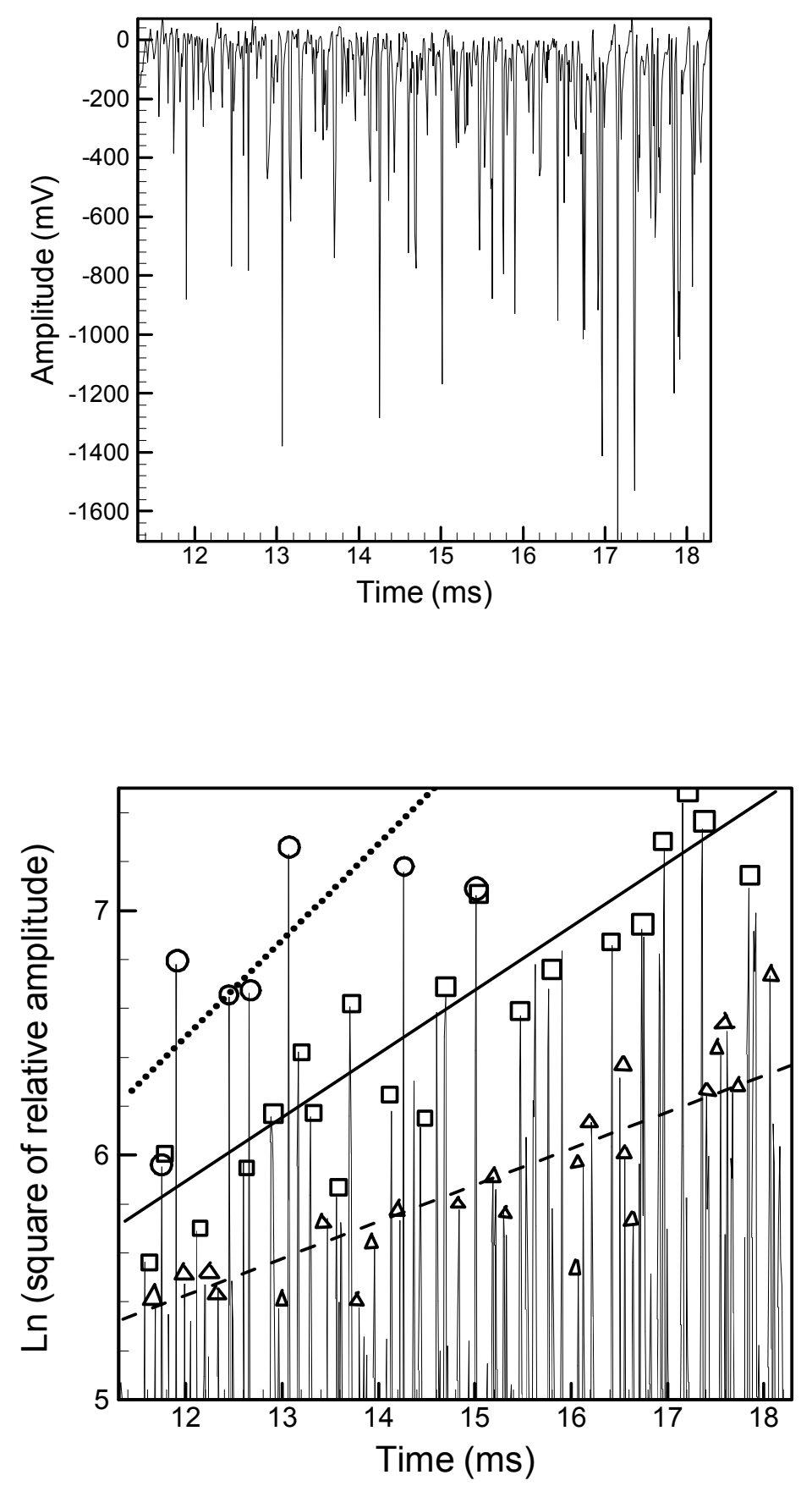

Fig. 61 (b) Pulse-extracted wave amplitude (up) and the least square fit of an exponential through the peaks (symbols) of the envelope (down). 
Table 5

\begin{tabular}{|c|c|l|l|l|l|l|l|}
\hline North hot plate & South hot plate & North hot & South hot & $\mathrm{Kz}$ & $\mathrm{Ky}$ & lon drift & Shear \\
\hline power $(\mathrm{kW})$ & power $(\mathrm{kW})$ & plate bias $(\mathrm{V})$ & plate bias $(\mathrm{V})$ & $(1 / \mathrm{cm})$ & $(1 / \mathrm{cm})$ & $(\mathrm{m} / \mathrm{s})$ & $1 / \mathrm{ms}$ \\
\hline 2.81 & 2.92 & -30 & 0 & 0.27 & 0.13 & 440 & -51.3 \\
2.72 & 2.8 & 0 & -30 & -0.56 & -0.33 & 620 & -45.7 \\
\hline
\end{tabular}
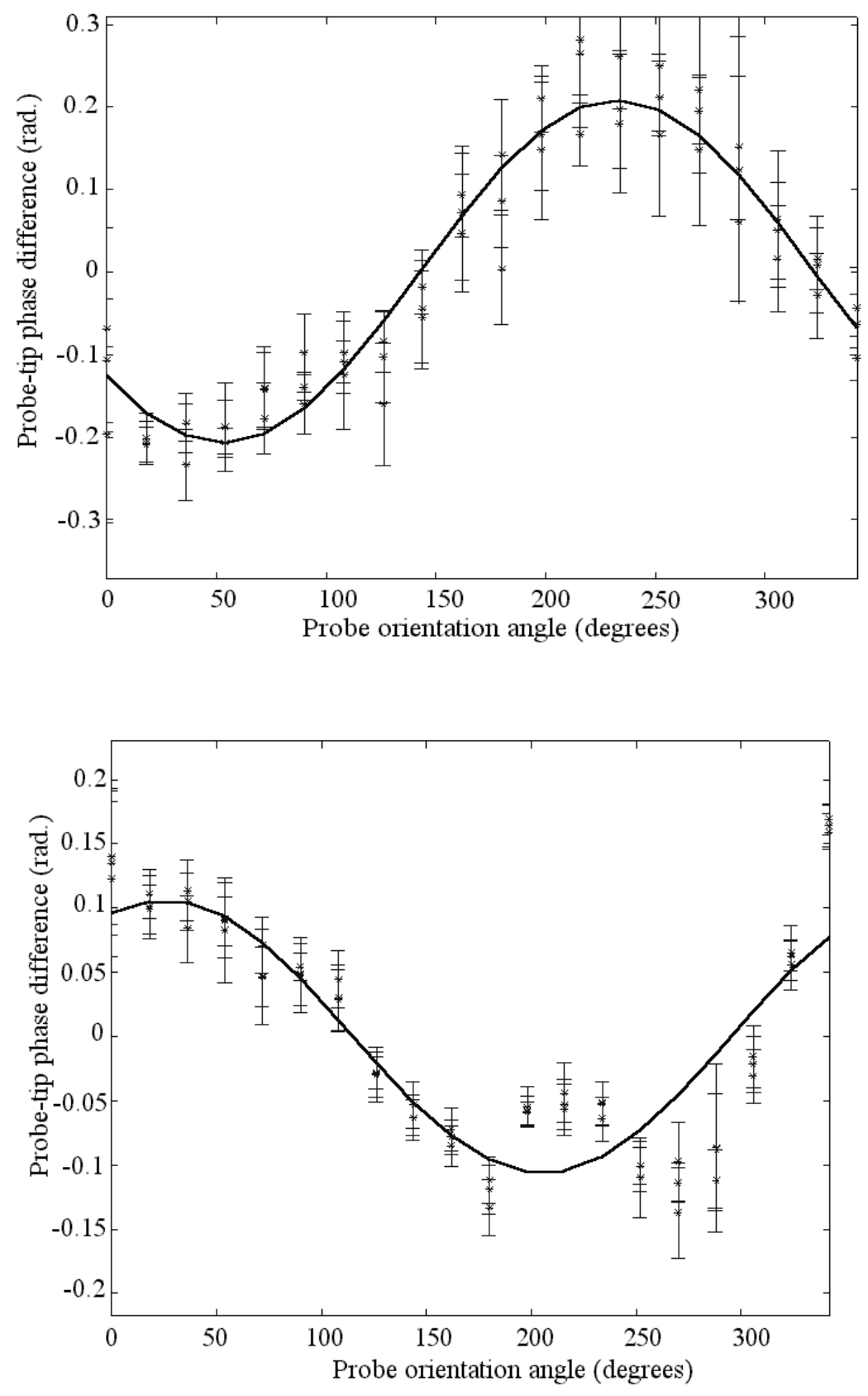

Fig. 62 Control over SMIA waves propagation direction using the ionizer relative bias. 


\begin{tabular}{|l|l|l|l|l|l|l|l|}
\hline North hot & South hot & North hot & South hot & Kz & Ky & lon drift & Shear \\
\hline plate power (kW & plate power $(\mathrm{kW})$ & plate bias (V) & plate bias $(\mathrm{V})$ & $(1 / \mathrm{cm})$ & $(1 / \mathrm{cm})$ & $(\mathrm{m} / \mathrm{s})$ & $1 / \mathrm{ms}$ \\
\hline 2.7 & 3.3 & -10 & 0 & -0.35 & -0.47 & 450 & -38.1 \\
3.2 & 2.6 & -10 & 0 & 0.29 & 0.52 & 560 & -41.3 \\
\hline
\end{tabular}

Table 6
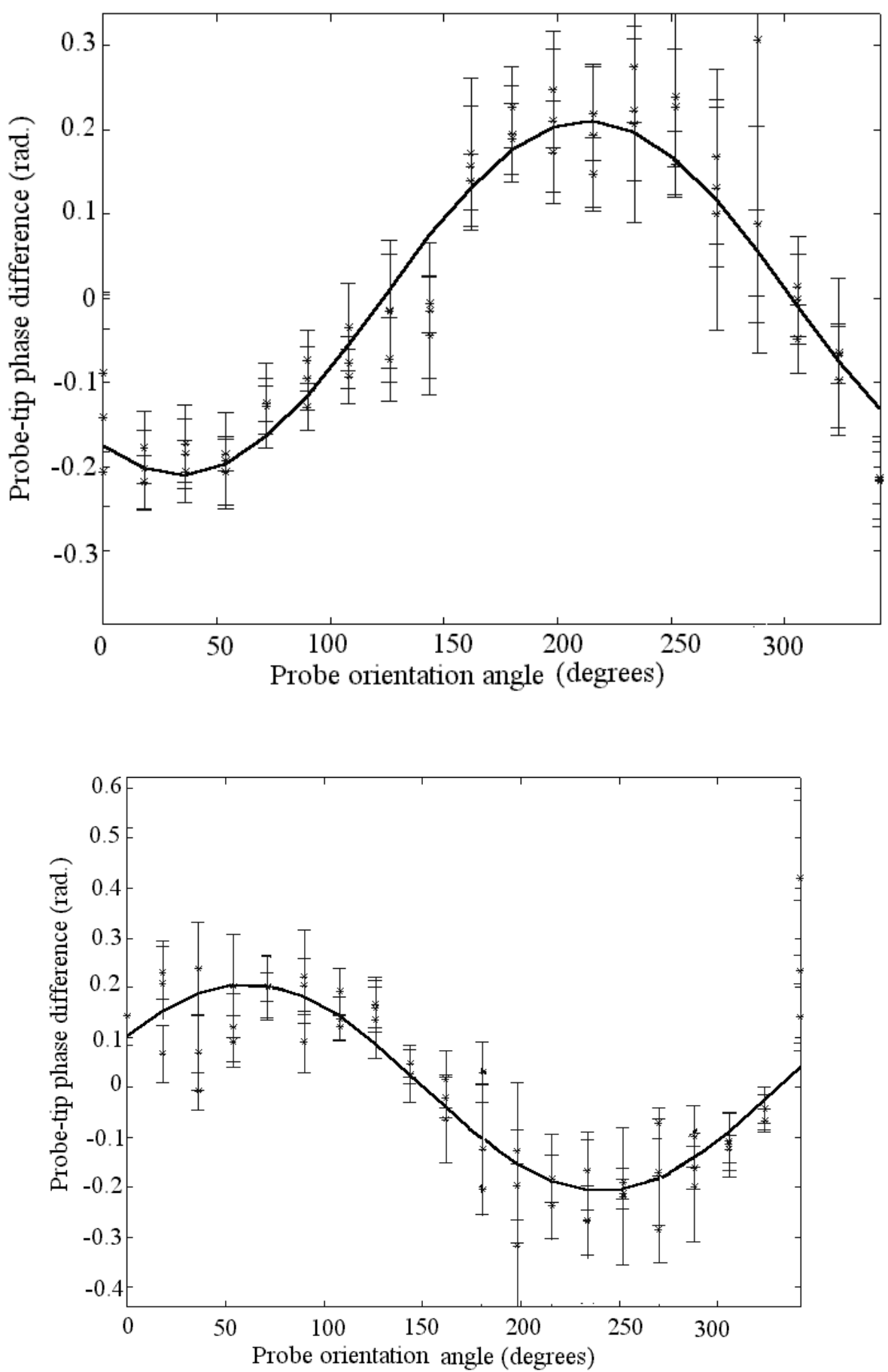

Fig. 63 Control over SMIA waves propagation direction using the ionizer emitting power. 


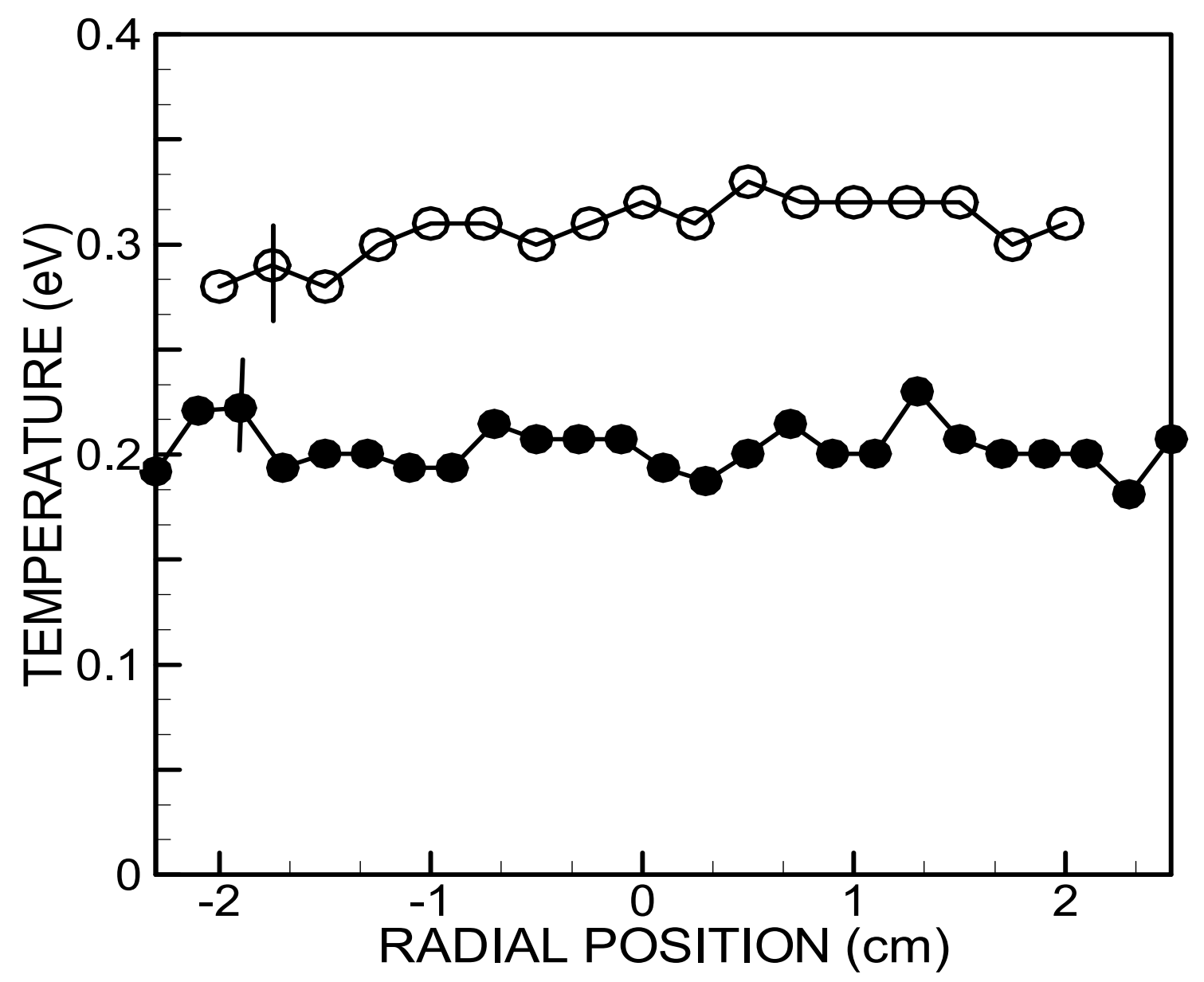

Fig. 64 LIF measured parallel ion temperature (filled circles) and perpendicular ion temperature (open circles) at the axial position $z=46 \mathrm{~cm}$. 


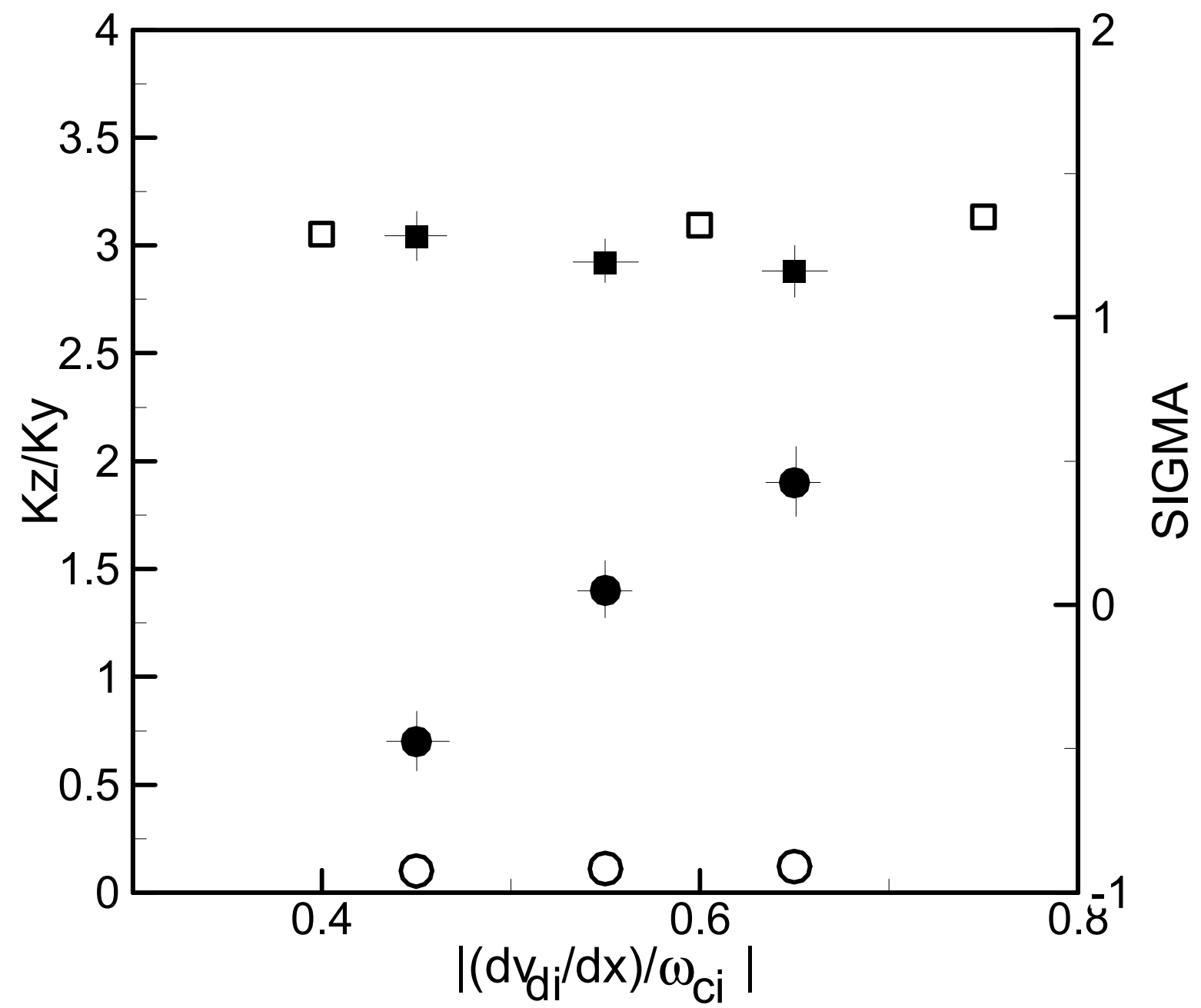

Fig. 65 Experimentally determined SMIA waves propagation angle in anisotropic ion-temperature plasma (filled circles)and optimum-growth predicted propagation angle in isotropic plasma (open circles) as functions of shear magnitude. Filled squares and open squares are the measured and calculated values of $\sigma$. Parameter values are listed in Table 7. 


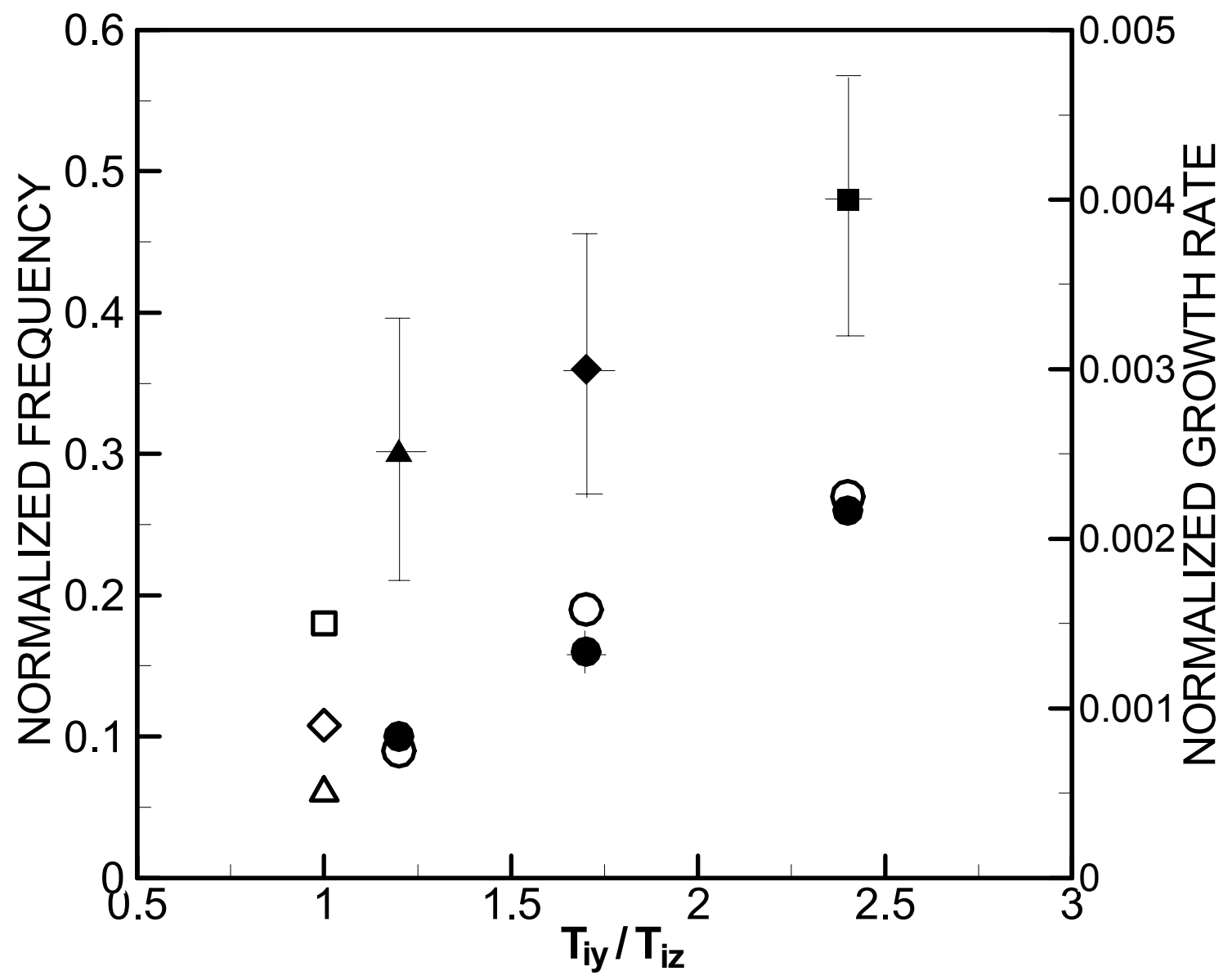

Fig. 66 SMIA waves growth rate (filled triangle, diamond and square, respectively) and frequency dependency (filled circles) on ion temperature anisotropy. Theoretically-predicted optimum growth rates (open triangle, diamond and square) in isotropic plasma. Shear values are $-0.45 \omega_{\mathrm{ci}}$ (triangle), $-0.54 \omega_{\mathrm{ci}}$ (diamond) and $-0.62 \omega_{\mathrm{ci}}$ (square), respectively. 

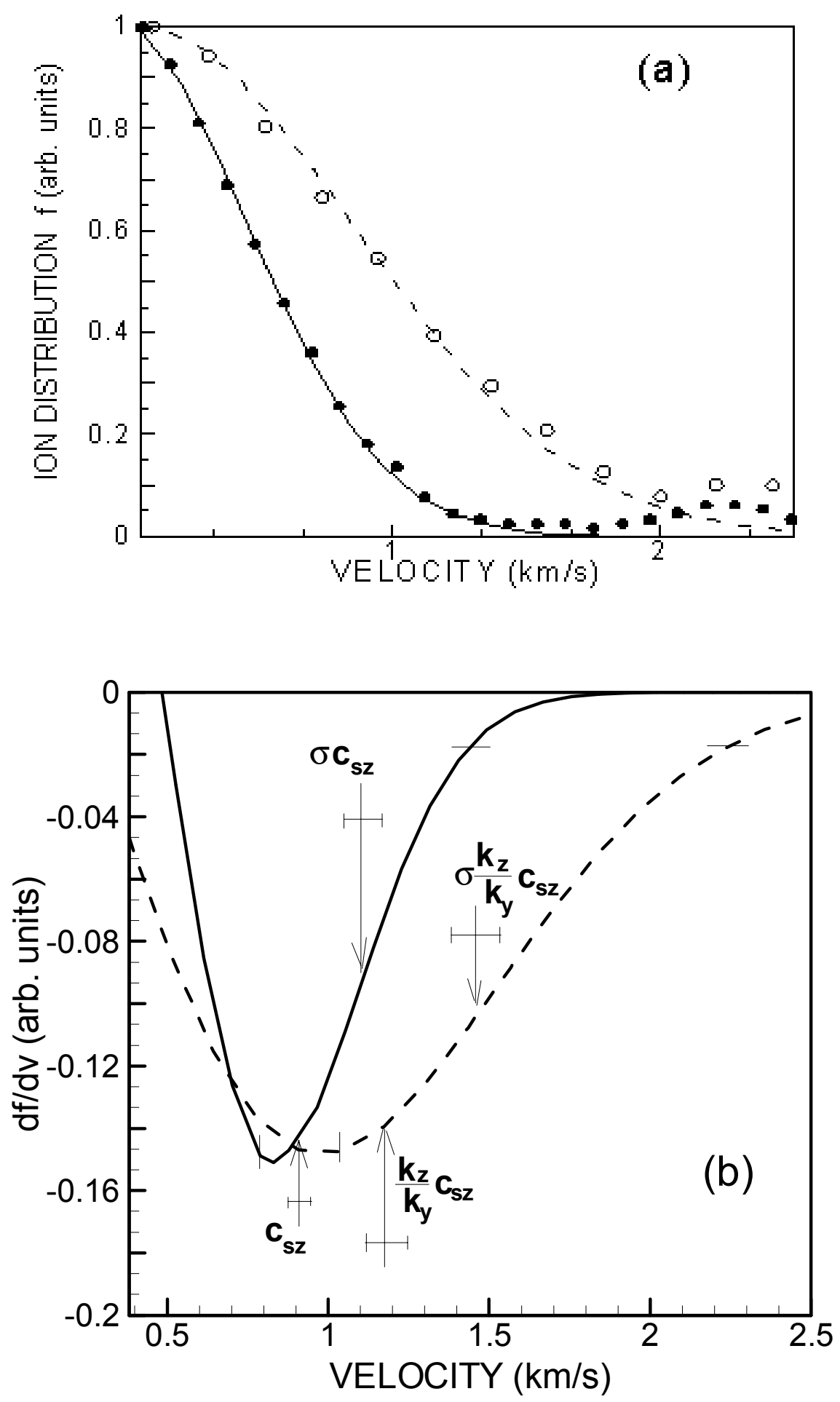

Fig. 67 (a) Parallel (filled circles) and perpendicular (open circles) LIF spectra and their corresponding fitted Maxwellian velocity distributions (parallel distribution solid line; perpendicular distribution - dashed line). (b) Derivatives of parallel (solid line) and perpendicular (dashed line) velocity distributions and the measured wave phase velocities, respectively. 


\begin{tabular}{|l|l|l|l|l|l|l|l|l|l|l|}
\hline $\begin{array}{l}\mathrm{T}_{\mathrm{iy}} \\
(\mathrm{eV})\end{array}$ & $\begin{array}{l}\mathrm{T}_{\mathrm{iz}} \\
(\mathrm{eV})\end{array}$ & $\begin{array}{l}\mathrm{T}_{\mathrm{e}} \\
(\mathrm{eV})\end{array}$ & $\begin{array}{l}\omega_{\mathrm{ci}}\left(10^{3}\right. \\
\mathrm{rad} / \mathrm{s})\end{array}$ & $\begin{array}{l}\mathrm{dv}_{\mathrm{di}} / \mathrm{dx}^{-1} \\
(\mathrm{~ms})^{-1}\end{array}$ & $\begin{array}{l}\mathrm{k}_{\mathrm{y}} \\
\left(\mathrm{cm}^{-1}\right)\end{array}$ & $\begin{array}{l}\mathrm{k}_{\mathrm{z}} \\
\left(\mathrm{cm}^{-1}\right)\end{array}$ & $\begin{array}{l}\omega_{\mathrm{r}}\left(10^{3}\right. \\
\mathrm{rad} / \mathrm{s})\end{array}$ & $\begin{array}{l}\omega_{\mathrm{i}}\left(10^{2}\right. \\
\mathrm{rad} / \mathrm{s})\end{array}$ & $\begin{array}{l}\omega\left(10^{3}\right. \\
\mathrm{rad} / \mathrm{s})\end{array}$ & $\begin{array}{l}\mathrm{v}_{\mathrm{di}} \\
(\mathrm{m} / \mathrm{s})\end{array}$ \\
\hline 0.27 & 0.22 & 0.33 & 96.7 & -45 & 0.15 & 0.10 & 9.6 & 2.4 & 14.4 & 480 \\
\hline 0.36 & 0.21 & 0.41 & 96.7 & -54 & 0.12 & 0.20 & 15.2 & 3.1 & 24.4 & 462 \\
\hline 0.46 & 0.19 & 0.51 & 96.7 & -62 & 0.11 & 0.23 & 28.8 & 3.9 & 39.3 & 460 \\
\hline
\end{tabular}

Table 7
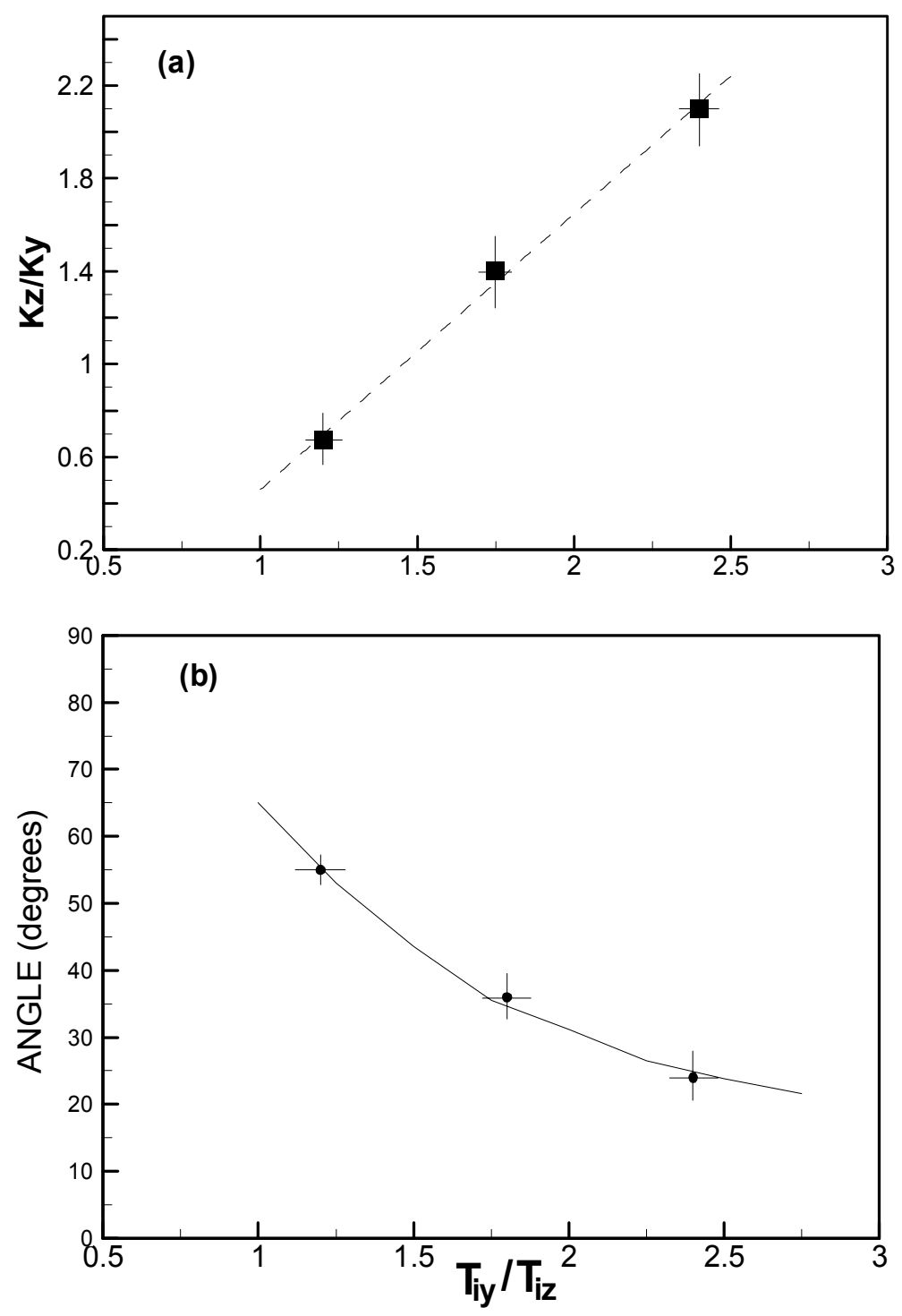

Fig. 68 SMIA waves propagation angle in anisotropic ion-temperature plasma. 


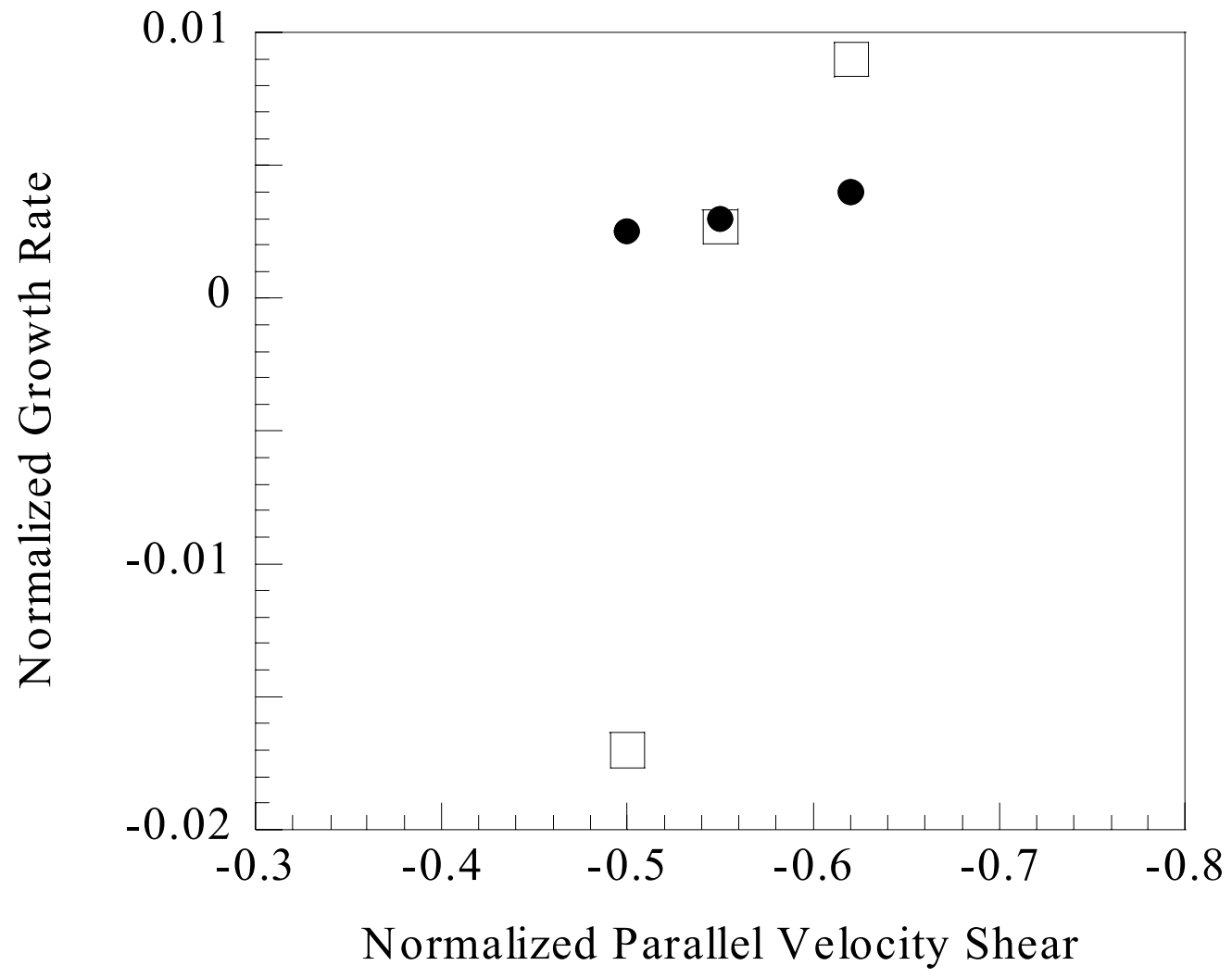

Fig. 69 Comparison between experimentally-measured SMIA wave growth rate (circles) in anisotropic barium-plasma and theory predicted (squares) growth rates for analogous plasma parameters (reprinted from [Scime et al., 2002]). 


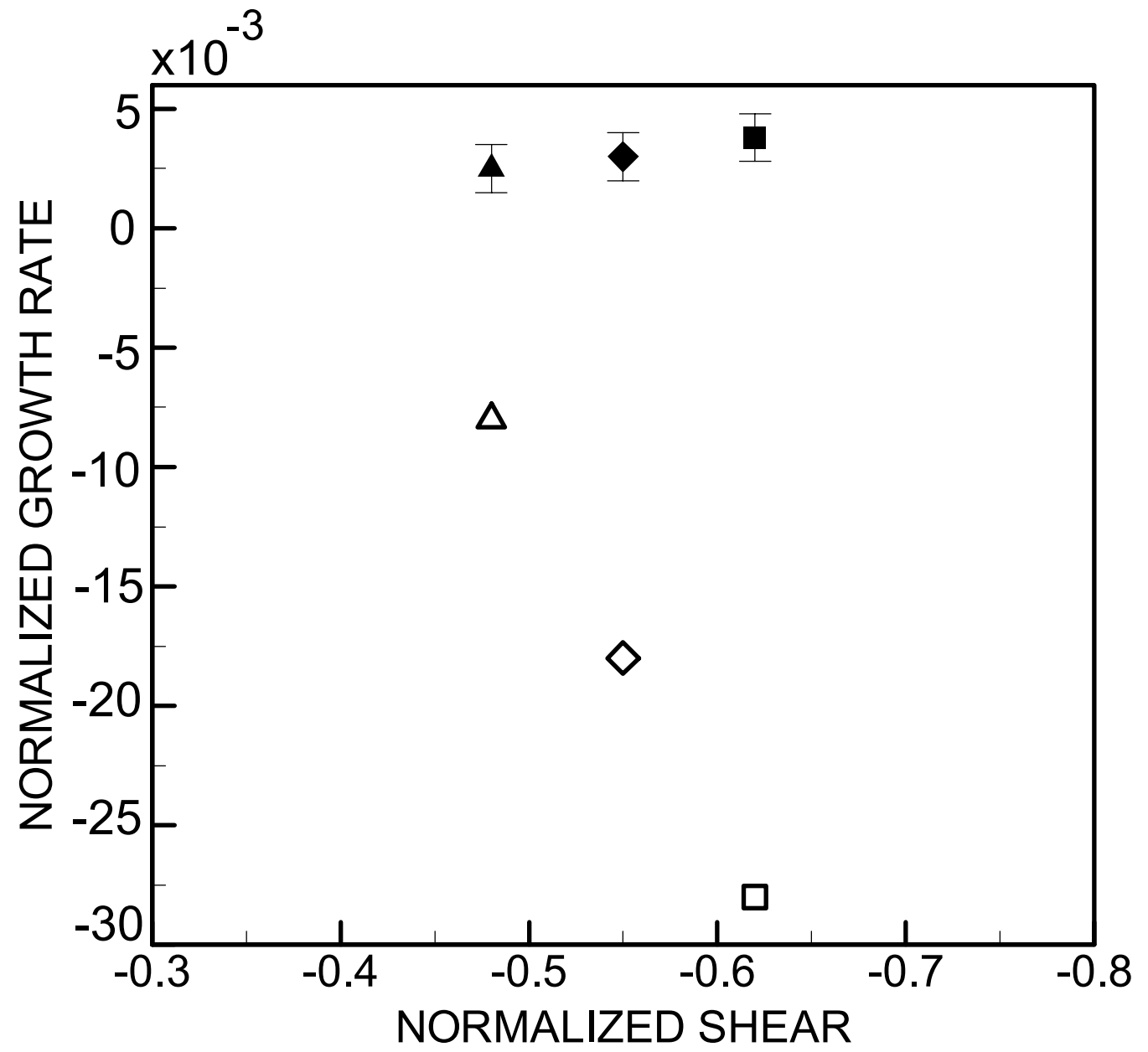

Fig. 70 Experimentally determined SMIA waves growth rate (filled symbols) in anisotropic ion-temperature barium plasma for $u=0.7$ (triangle), 1.4 (diamond), 1.9 (square) and theoretically predicted values (open symbols) in isotropic barium plasma for identical propagation angles. 

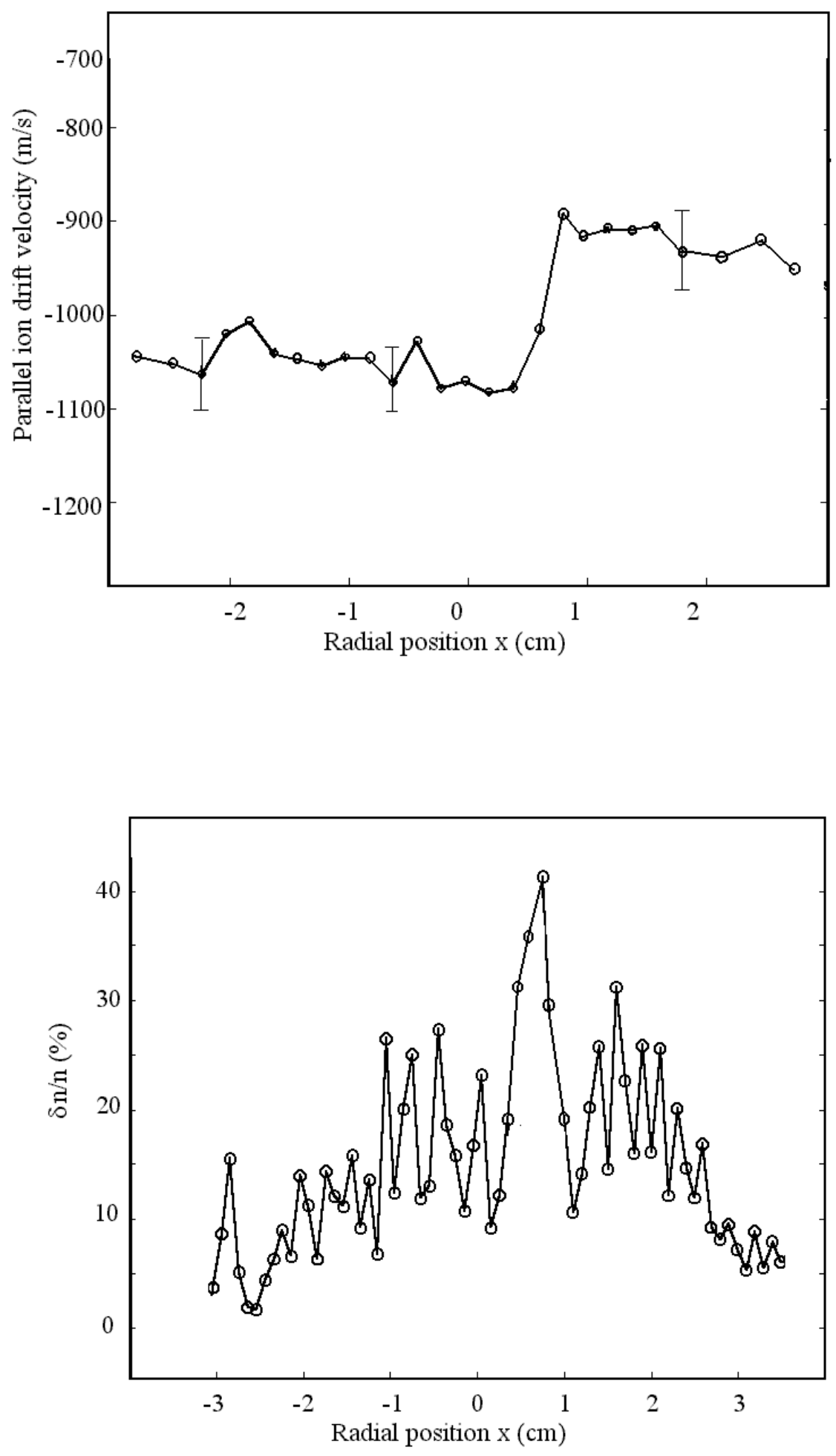

Fig. 71 (a) Parallel ion drift velocity radial profile and (b) the shear-destabilized D'Angelo instability amplitude radial profile (inferred from ion saturation current fluctuations on a Langmuir probe). Here $B=0.11 \mathrm{~T}$ (southward), $V_{N}=-4 \mathrm{~V}$, and $V_{S}=10 \mathrm{~V}$. 


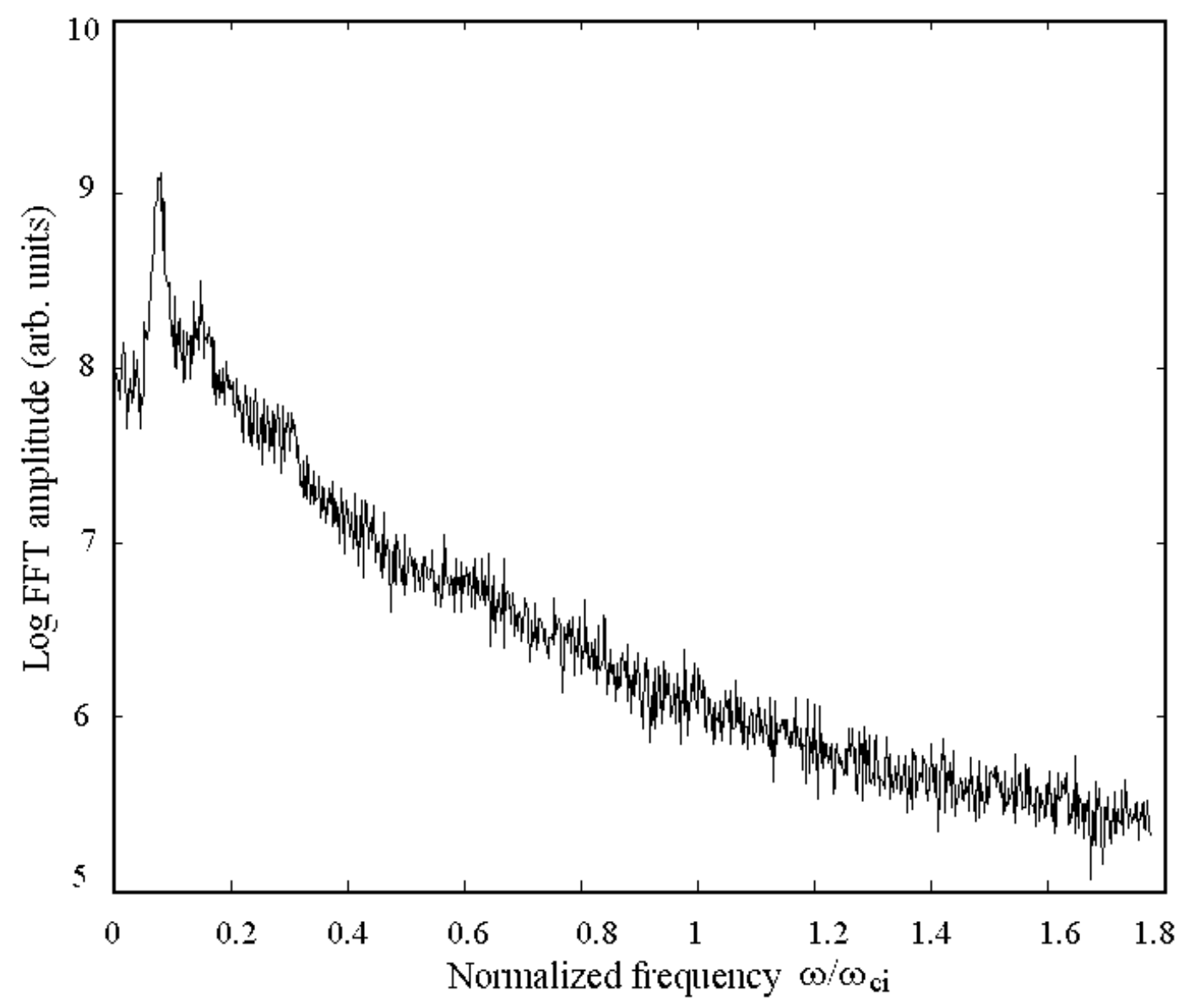

Fig. 72 D'Angelo instability normalized spectrum. 


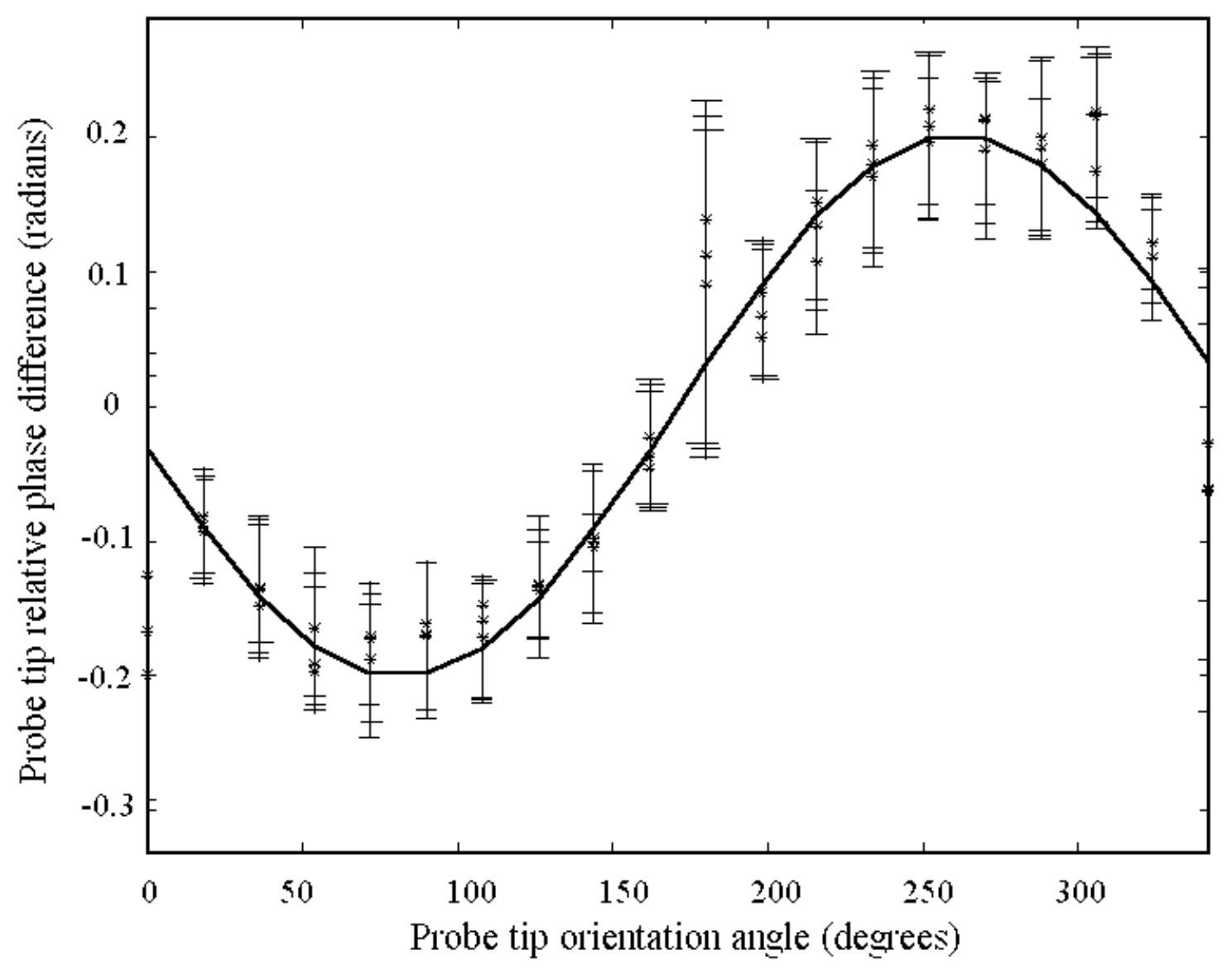

Fig. 73 The D'Angelo instability relative phase difference between probe tips as a function of probe orientation. The fitted sinusoid yields $k_{z}=-0.07 \mathrm{~cm}^{-1}$ and $k_{y}=-0.75 \mathrm{~cm}^{-1}$. 


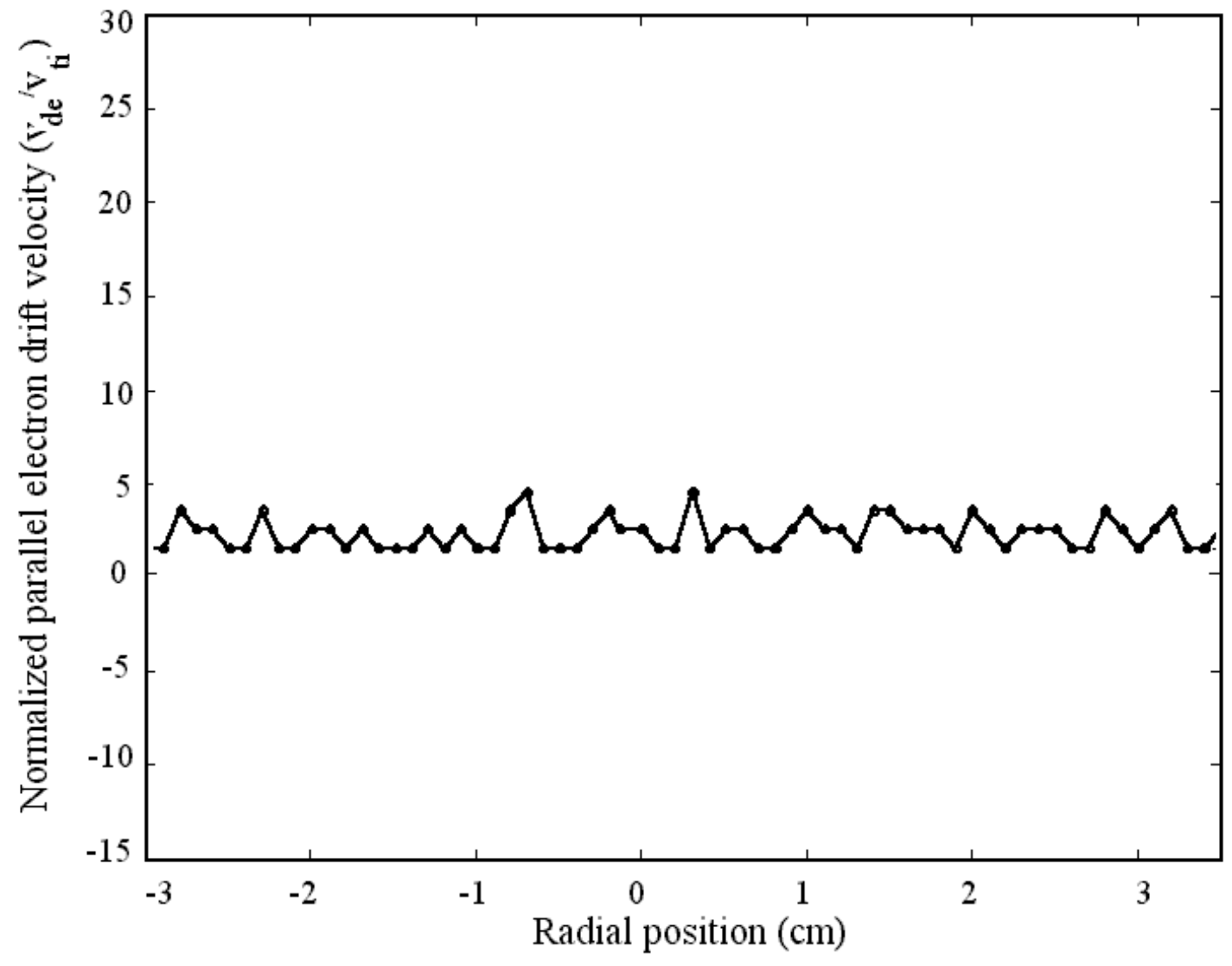

Fig. 74 Parallel-electron-drift velocity radial profile in the presence of the D'Angelo instability. 


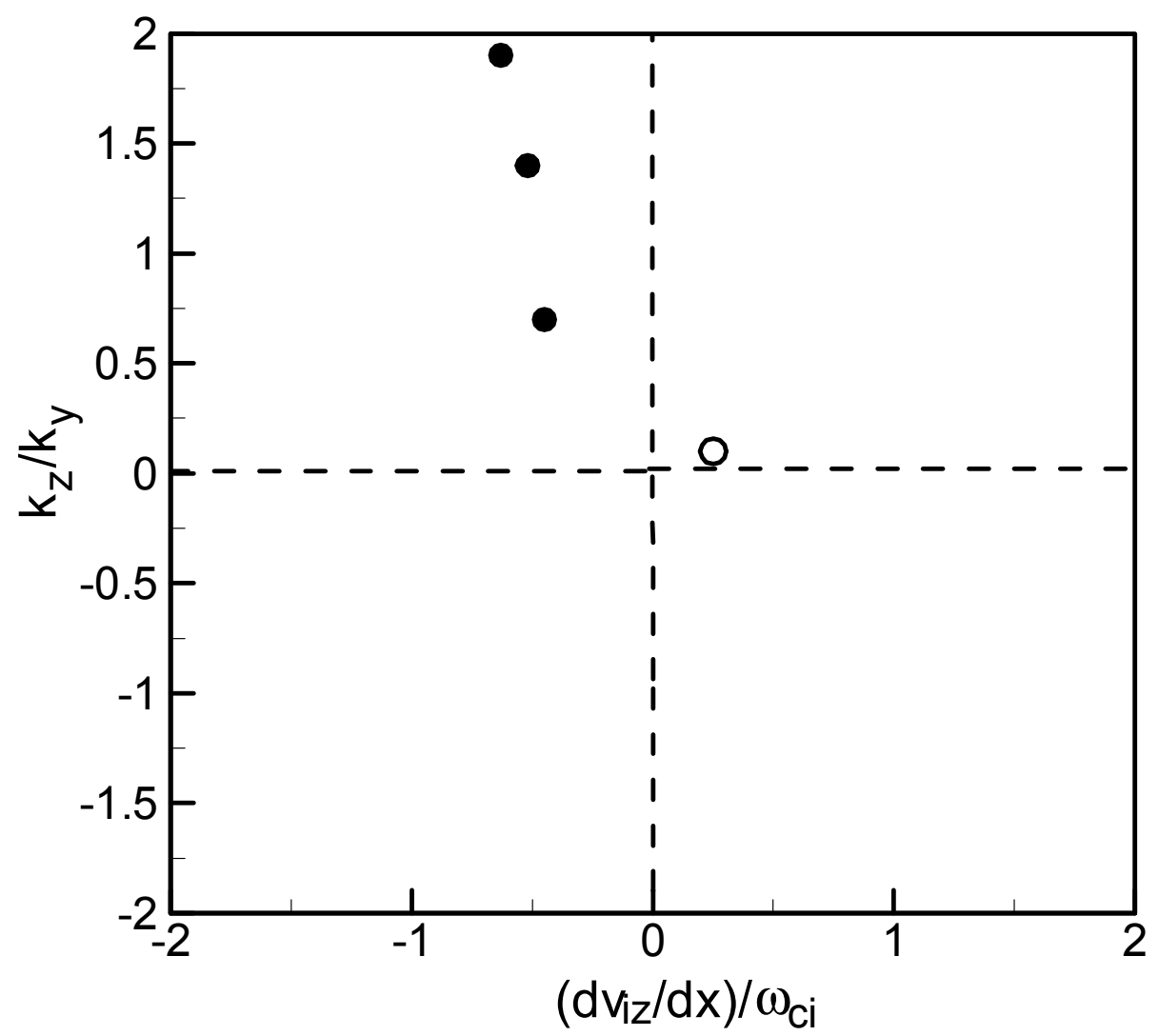

Fig.75 Low-frequency waves regimes in the presence of parallel-velocity shear: first and third quadrants - the D'Angelo instability, second and fourth quadrants SMIA waves. Circles represent experimental data. 


\section{Appendix 1}

$\%$ Gaussfit \%

\% Created by E. Reynolds (June 2001); modified by C. Teodorescu (July 2001)

\%This MATLAB program fits a lineshape to the L.I.F. signal obtained using the perpendicular \%injection optics. Based on the lineshape, it then determines the best-fit Maxwellian $\%$ distribution and its parameters: perpendicular ion-temperature and perpendicular $\%$ ion drift velocity.

\% The program is set up to analyze L.I.F. data obtained with both the input polarizer $\%$ and the collection optics polarizer aligned with the magnetic field

function $[\mathrm{T}, \mathrm{vd}]=$ gausfit(lif0,calib,Bdial,scanwidth,crop)\% reads the raw lif data, index of max in calib.signal, $\%$ seperation of peaks in $\mathrm{m} / \mathrm{s}$, scanwidth in GigaHertz, crop indexes

global liff sep v \%definition of variables

liff=lif0;

$\operatorname{lifO}(1: \operatorname{crop}(1))=0 ; \% \operatorname{lif0}(\operatorname{crop}(2):$ lifres $)=0 ; \%$ crop data

load('persep'); $\mathrm{p}=$ polyfit(B,persep, 1$) ; \mathrm{sep}=\mathrm{p}(1) * \mathrm{Bdial}+\mathrm{p}(2) ; \%$ loads the file that

$\mathrm{m}=137 ; \% \mathrm{mass}$ in AMU $\quad \%$ sets the line separation (as function on B).

$\mathrm{k}=1.6 \mathrm{e}-19 ; \mathrm{s}=\operatorname{size}(\mathrm{lif0}) ; \mathrm{lifres}=\mathrm{s}(1) ; \%$ Boltzmann constant, size of data array and lif resolution

$\mathrm{amu}=1.66 \mathrm{e}-27$; \%atomic mass unit

$\mathrm{v}=[$-calib+1:lifres-calib]'.*scanwidth*585/lifres; \%velocity axis scale

lambda $=585.5301+\mathrm{v} . * 0.0381 ; \%$ zero-velocity reference wavelength

$\mathrm{dv}=$ scanwidth $* 585 ; \%$ velocity resolution

$\mathrm{A}=\max (\mathrm{lif0}) ; \%$ initial amplitude guess

$\mathrm{vd}=\operatorname{sum}($ lif0.*v)/sum(lif0);\%initial guess for drift

$\mathrm{T}=.2 ; \%$ initial temperature guess in $\mathrm{eV}$

signal $=\mathrm{gsn}(\mathrm{A}, \mathrm{vd}+\operatorname{sep} / 2, \mathrm{v}, \mathrm{T})+\mathrm{gsn}(\mathrm{A}, \mathrm{vd}-\operatorname{sep} / 2, \mathrm{v}, \mathrm{T}) ; \%$ first guess

$\mathrm{dx}=.04 ; \mathrm{up}=1+\mathrm{dx} ; \mathrm{dn}=1-\mathrm{dx} ; \%$ increment variables

\%three parameter fit: drift velocity, temperature, signal amplitude using the least square method; the loop is done 200 times.

for $\mathrm{i}=1: 200$;

$\left.\mathrm{dA}=\operatorname{sum}\left(\left(\mathrm{gsn}\left(\mathrm{A}^{*} u p, \mathrm{vd}+\operatorname{sep} / 2, \mathrm{v}, \mathrm{T}\right)+\mathrm{gsn}\left(\mathrm{A}^{*} \mathrm{up}, \mathrm{vd}-\mathrm{sep} / 2, \mathrm{v}, \mathrm{T}\right)-\mathrm{lif0}\right)\right)^{\wedge} 2\right)-$

$\operatorname{sum}\left(\left(g \operatorname{sn}\left(A^{*} d n, v d+\operatorname{sep} / 2, v, T\right)+g \operatorname{sn}(A * d n, v d-s e p / 2, v, T)-l i f 0\right) .^{\wedge} 2\right)$; 


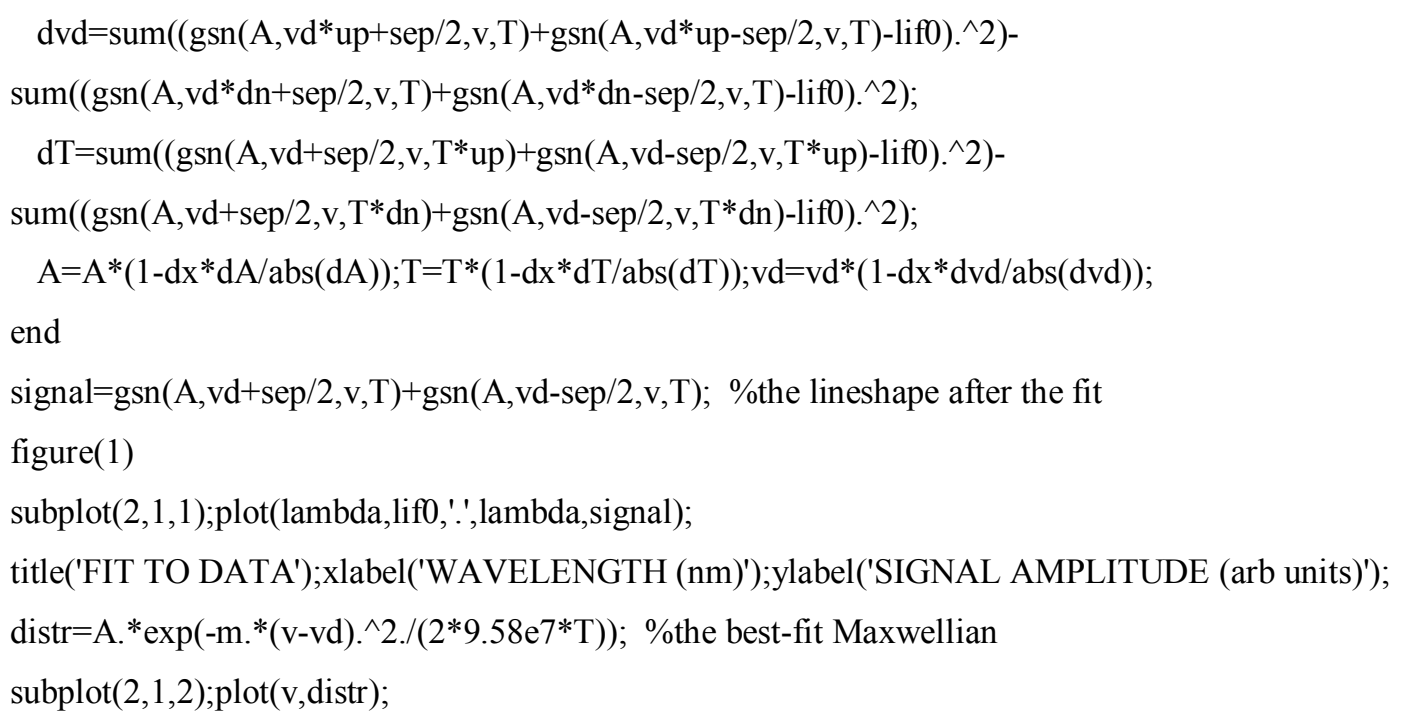

$\%$ plot of $\mathrm{df} / \mathrm{dv}$

$\mathrm{fz}=$ distr./max(distr); \%normalized distribution figure(3)

plot((v(1:99),diff(fz),'-.');

xlabel('Drift velocity (m/s)');ylabel('df/dv (arb. units)'); 


\section{Appendix 2}

$\%$ Gausfit $2 \%$

\% Created by E. Reynolds (June 2001); modified by C. Teodorescu (July 2001)

\%This MATLAB program fits the best lineshape to the L.I.F. signal acquired using

$\%$ the parallel injection optics. Based on the lineshape, it then determines

\%the best-fit Maxwellian distibution and the parameters: parallel-ion-drift

$\%$ velocity and parallel ion temperature.

\% This fit is for parallel LIF with only four sigma lines present with Zeeman split

$\%$ and line ratios taken from experimental data in Matlab workspace 01jul09a.mat

\%Positive velocity indicates drift along B (i.e., in the south direction)

function $[\mathrm{A}, \mathrm{vd}, \mathrm{T}]=$ gausfit2(lifd,calib,Bdial,scanwidth,crop)\%raw lif data, index of max in calib.signal, \%magnetic field dial setting, scanwidth in GigaHertz, crop is an array of two index values

\%outside of which the data is set to zero

global liff sep v lineratio

liff=lifd;

siz=size(lifd);lifres $=\operatorname{siz}(1) ; \%$ lif resolution

$\mathrm{k}=1.6 \mathrm{e}-19 ; \mathrm{amu}=1.66 \mathrm{e}-27 ; \mathrm{m}=137 ; \%$ mass in AMU

$\operatorname{lifd}(1: \operatorname{crop}(1))=0 ; \operatorname{lifd}(\operatorname{crop}(2):$ lifres $)=0 ; \%$ crop data

load('01jul09a.mat');sep=interp1(b,sigma2,Bdial);\%experimentally measured Zeeman split

$\mathrm{v}=-[-$ calib $+1:$ lifres-calib]'.*scanwidth $* 585 /$ lifres;\%velocity axis

lambda $=585.5301+\mathrm{v} . * 0.000171 /$ lifres;

$\mathrm{dv}=$ scanwidth $* 585 /$ lifres; \% velocity resolution

$\mathrm{A}=\max ($ lifd $) / 2 ; \%$ initial amplitude guess

lineratio $=5 / 6 ; \%$ amplitude ratio of inner to outer lines

$\mathrm{vd}=\operatorname{sum}\left(\right.$ lifd. $\left.^{*} \mathrm{v}\right) /$ sum(lifd);\%initial geuss for drift

$\mathrm{T}=.2 ; \%$ initial temperature guess in $\mathrm{eV}$

signal=plifsignal $(\mathrm{A}, \mathrm{vd}, \mathrm{T}) ; \%$ first guess

$\mathrm{dx}=.01 ; \mathrm{up}=1+\mathrm{dx} ; \mathrm{dn}=1-\mathrm{dx} ; \%$ increment variables

\%three parameter fit: parallel drift velocity, temperature, signal amplitude, based on the least square method

for $\mathrm{i}=1: 300$;

$\mathrm{dA}=\operatorname{sum}\left(\left(\right.\right.$ plifsignal(A*up,vd,T)-lifd).^2)-sum((plifsignal $\left.\left.\left(\mathrm{A}^{*} \mathrm{dn}, \mathrm{vd}, \mathrm{T}\right)-\mathrm{lifd}\right) .^{\wedge} 2\right) ;$

$\mathrm{A}=\mathrm{A} *(1-\mathrm{dx} * \mathrm{dA} / \mathrm{abs}(\mathrm{dA}))$;

$\mathrm{A} 2=\mathrm{A} * 5 / 6$; 
$\mathrm{dvd}=\operatorname{sum}\left(\left(\operatorname{plifsignal}\left(\mathrm{A}, \mathrm{vd} \mathrm{d}^{*} \mathrm{up}, \mathrm{T}\right)-\mathrm{lifd}\right) .^{\wedge} 2\right)$-sum((plifsignal(A,vd*dn,T)-lifd).^2);

$v d=v d *(1-d x * d v d / a b s(d v d))$;

$\mathrm{dT}=\operatorname{sum}\left(\left(\operatorname{plifsignal}\left(\mathrm{A}, \mathrm{vd}, \mathrm{T}^{*} \mathrm{up}\right)-\right.\right.$ lifd $\left.) . \wedge 2\right)-\operatorname{sum}\left(\left(\operatorname{plifsignal}\left(\mathrm{A}, \mathrm{vd}, \mathrm{T}^{*} \mathrm{dn}\right)-\right.\right.$ lifd $\left.) . \wedge 2\right)$;

$\mathrm{T}=\mathrm{T} *(1-\mathrm{dx} * \mathrm{dT} / \mathrm{abs}(\mathrm{dT}))$;

end;

$\% \mathrm{vd}=\mathrm{vd}+20$;

signal=plifsignal(A,vd,T); \%best fit lineshape

figure(1);

subplot $(2,1,1)$;

plot(lambda,lifd./A,'.',lambda,signal./A,'r');

axis([lambda(lifres) lambda(1) $0 \max (\text { lifd })^{*} 1.1 /$ A] $)$;

title('FIT TO DATA');

xlabel('LASER WAVELENGTH (nm)');ylabel('SIGNAL AMPLITUDE (arb units)');

distr $=\mathrm{A} . * \exp (-\mathrm{m} . *(\mathrm{v}-\mathrm{vd}) . \wedge 2 . /(2 * 9.58 \mathrm{e} 7 * \mathrm{~T})) ; \%$ best fit Maxwellian distribution

subplot(2,1,2);plot(v,distr./max(distr));

$\operatorname{axis([v(lifres)} \mathrm{v}(1) \quad 01.1])$;

title('THE BEST GAUSSIAN REPRESENTATION OF THE DATA');

xlabel('VELOCITY (M/S)');ylabel('SIGNAL AMPLITUDE (arb units)');

temp $=$ sprintf('ION TEMP $=\%$ f eV',T)

vdrift=sprintf('ION DRIFT \% $\left.\mathrm{f} \mathrm{m} / \mathrm{s}^{\prime}, \mathrm{vd}\right)$

\%text(v(lifres),5/6,temp);\%text(v(lifres),4/6,vdrift);

$\%$ plot of $\mathrm{df} / \mathrm{dv}$

$\mathrm{fz}=$ distr./max (distr);

figure(3)

$\operatorname{plot}(\mathrm{v}(1: 99), \operatorname{diff}(\mathrm{fz}))$;

xlabel('Drift velocity (m/s)');ylabel('df/dv (arb. units)'); 


\section{Appendix 3}

$\%$ Gaussfit $3 \%$

\% Created by E. Reynolds (July 2001); modified by C. Teodorescu (July 2001)

\%This MATLAB program fits the best lineshape to the L.I.F. signal acquired using the parallel $\%$ injection optics. Based on the lineshape, it then determines the best-fit two-Maxweellian \%distribution and the parameters: parallel ion drift velocity of the direct-streaming population, $\%$ the parallel drift velocity of the counter-streaming population and their temperature. $\%$ This fitting routine has five parameters to allow for a counterstreaming population $\%$ temperatures of the two populations are assumed equal \% This fit is for parallel LIF with only four sigma lines present with Zeeman split $\%$ and line ratios taken from experimental data in Matlab workspace 01jul09a.mat

function $[\mathrm{A}, \mathrm{vd}, \mathrm{A} 2, \mathrm{vd} 2, \mathrm{~T}]=$ gausfit3(lifd,calib,Bdial,scanwidth,crop)\%raw lif data, index of max in calib.signal, \%magnetic field dial setting, scanwidth in GigaHertz, crop is an array of two index values $\%$ outside of which the data is set to zero

global liff sep v lineratio

lineratio $=5 / 6 ; \%$ amplitude ratio of inner to outer lines

liff=lifd;

$\operatorname{siz}=\operatorname{size}($ lifd $) ;$ lifres $=\operatorname{siz}(1) ; \%$ lif resolution

$\mathrm{k}=1.6 \mathrm{e}-19 ; \mathrm{amu}=1.66 \mathrm{e}-27 ; \mathrm{m}=137 ; \%$ mass in AMU

$\operatorname{lifd}(1: \operatorname{crop}(1))=0 ; \operatorname{lifd}(\operatorname{crop}(2): \operatorname{lifres})=0 ; \%$ crop data

load('01jul09a.mat'); $=$ polyfit(b,SIGMA2,1);sep=p(1)*(600+Bdial)+p(2);sep=interp1(b,sigma2,Bdial);\%experi mentally measured Zeeman split is loaded

$\mathrm{v}=-[-$ calib+1:lifres-calib]'.*scanwidth*585/lifres;\%velocity axis

lambda $=585.5301+\mathrm{v} . * 0.000171 /$ lifres;

$\%[\mathrm{~A}, \mathrm{vd}, \mathrm{T}]=$ gausfit2(lifd,calib,Bdial,scanwidth,crop);

$\mathrm{A}=\max ($ lifd $) / 2 ; \%$ initial amplitude guess

$\mathrm{vd}=$ sum(lifd. $\left.{ }^{\mathrm{v}} \mathrm{v}\right) / \mathrm{sum}($ lifd+eps $) ; \mathrm{vd}=-500 ; \%$ initial guess for drift of population 1

$\mathrm{T}=.2 ; \%$ initial temperature guess in $\mathrm{eV}$

$\mathrm{A} 2=1.0 * \mathrm{~A} ; \%$ This is an initial guess you must make for the relative density of the second population $\mathrm{vd} 2=-\mathrm{vd} ; \%$ initial guess for drift of the second population signal=plifsignal $(\mathrm{A}, \mathrm{vd}, \mathrm{T})+\operatorname{plifsignal}(\mathrm{A} 2, \mathrm{vd} 2, \mathrm{~T}) ; \%$ first guess 
$\mathrm{dx}=.03 ; \mathrm{up}=1+\mathrm{dx} ; \mathrm{dn}=1-\mathrm{dx} ; \%$ increment variables

\%five parameter fit: parallel drift velocity of population \#1, temperature, signal amplitude

$\%$ of population \#1, parallel drift velocity of population\#2 and signal amplitude of population \#2

\%using least square method

for $\mathrm{i}=1: 200$;

$\mathrm{dA}=\operatorname{sum}\left(\left(\operatorname{plifsignal}\left(\mathrm{A}^{*} \mathrm{up}, \mathrm{vd}, \mathrm{T}\right)+\operatorname{plifsignal}(\mathrm{A} 2, \mathrm{vd} 2, \mathrm{~T})-\mathrm{lifd}\right) .^{\wedge} 2\right)-$

$\operatorname{sum}\left(\left(\operatorname{plifsignal}\left(\mathrm{A}^{*} \mathrm{dn}, \mathrm{vd}, \mathrm{T}\right)+\operatorname{plifsignal}(\mathrm{A} 2, \mathrm{vd} 2, \mathrm{~T})-\mathrm{lifd}\right) .{ }^{\wedge} 2\right)$;

$\mathrm{A}=\mathrm{A} *(1-\mathrm{dx} * \mathrm{dA} / \mathrm{abs}(\mathrm{dA}+\mathrm{eps}))$;

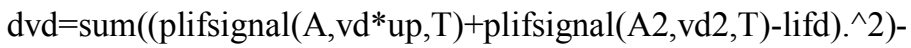

$\operatorname{sum}((\operatorname{plifsignal}(\mathrm{A}, \mathrm{vd} * \mathrm{dn}, \mathrm{T})+\operatorname{plifsignal}(\mathrm{A} 2, \mathrm{vd} 2, \mathrm{~T})-\mathrm{lifd}) . \wedge 2)$;

$\mathrm{vd}=\mathrm{vd} *(1-\mathrm{dx} * \mathrm{dvd} / \mathrm{abs}(\mathrm{dvd}+\mathrm{eps}))$;

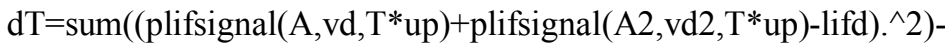

$\operatorname{sum}\left(\left(\operatorname{plifsignal}\left(\mathrm{A}, \mathrm{vd}, \mathrm{T}^{*} \mathrm{dn}\right)+\operatorname{plifsignal}\left(\mathrm{A} 2, \mathrm{vd} 2, \mathrm{~T}^{*} \mathrm{dn}\right)-\mathrm{lifd}\right) . \wedge 2\right) ;$

$\mathrm{T}=\mathrm{T} *(1-\mathrm{dx} * \mathrm{dT} / \mathrm{abs}(\mathrm{dT}+\mathrm{eps}))$;

$\mathrm{dA} 2=\operatorname{sum}((\operatorname{plifsignal}(\mathrm{A}, \mathrm{vd}, \mathrm{T})+\operatorname{plifsignal}(\mathrm{A} 2 * \mathrm{up}, \mathrm{vd} 2, \mathrm{~T})-\mathrm{lifd}) . \wedge 2)-$

$\operatorname{sum}\left((\right.$ plifsignal $\left.(\mathrm{A}, \mathrm{vd}, \mathrm{T})+\operatorname{plifsignal}(\mathrm{A} 2 * \mathrm{dn}, \mathrm{vd} 2, \mathrm{~T})-\mathrm{lifd}) .{ }^{\wedge} 2\right) ;$

$\mathrm{A} 2=\mathrm{A} 2 *(1-\mathrm{dx} * \mathrm{dA} 2 / \mathrm{abs}(\mathrm{dA} 2+\mathrm{eps}))$;

$\mathrm{dvd} 2=\operatorname{sum}((\operatorname{plifsignal}(\mathrm{A}, \mathrm{vd}, \mathrm{T})+\operatorname{plifsignal}(\mathrm{A} 2, \mathrm{vd} 2 * \mathrm{up}, \mathrm{T})-\mathrm{lifd}) . \wedge 2)-$

$\operatorname{sum}\left((\operatorname{plifsignal}(\mathrm{A}, \mathrm{vd}, \mathrm{T})+\operatorname{plifsignal}(\mathrm{A} 2, \mathrm{vd} 2 * \mathrm{dn}, \mathrm{T})-\mathrm{lifd}) .^{\wedge} 2\right)$;

$\mathrm{vd} 2=\mathrm{vd} 2 *(1-\mathrm{dx} * \mathrm{dvd} 2 / \mathrm{abs}(\mathrm{dvd} 2+\mathrm{eps})) ;$

end

signal=plifsignal $(\mathrm{A}, \mathrm{vd}, \mathrm{T})+\operatorname{plifsignal}(\mathrm{A} 2, \mathrm{vd} 2, \mathrm{~T})$;

subplot(2,1,1);plot(lambda,lifd./A,'.',lambda,signal./A);

axis([lambda(lifres) lambda(1) $0 \max ($ lifd)*1.5/A]);

\%title('FIT TO DATA');

xlabel('LASER WAVELENGTH (nm)');ylabel('SIGNAL AMPLITUDE (arb units)');

$\operatorname{distr} 1=\mathrm{A} .{ }^{*} \exp \left(-\mathrm{m} .^{*}(\mathrm{v}-\mathrm{vd}) .^{\wedge} 2 . /(2 * 9.58 \mathrm{e} 7 * \mathrm{~T})\right)$;

distr2=A2.*exp(-m.*(v-vd2).^2./(2*9.58e 7*T)); $=\max (\operatorname{distr} 1+\operatorname{distr} 2)$;

$\operatorname{subplot}(2,1,2) ; p \operatorname{plot}\left(\mathrm{v},(\operatorname{distr} 1+\operatorname{distr} 2) . / \mathrm{f}, \mathrm{v}, \operatorname{distr} 1 / \mathrm{f},{ }^{\prime} \mathrm{g} . ', \mathrm{v}, \operatorname{distr} 2 / \mathrm{f},{ }^{\prime} \mathrm{b} . '\right)$;

$\operatorname{axis}([\mathrm{v}($ lifres $) \mathrm{v}(1) \quad 01.1])$;

\%title('THE BEST GAUSSIAN REPRESENTATION OF THE DATA');

xlabel('VELOCITY (M/S)');ylabel('SIGNAL AMPLITUDE (arb units)');

temp $=$ sprintf('ION TEMP $=\% \mathrm{f} \mathrm{eV',T)}$;

vdrift $=$ sprintf('green ion drift $\left.=\% \mathrm{fm} / \mathrm{s}^{\prime}, \mathrm{vd}\right)$;

vdrift2 $=$ sprintf('blue ion drift $\left.=\% \mathrm{f} \mathrm{m} / \mathrm{s}^{\prime}, \mathrm{vd} 2\right)$;

relamp $=\operatorname{sprintf('relative~density~of~blue~}=\% \mathrm{f}, \mathrm{A} 2 / \mathrm{A})$; 
text(v(lifres), $1 / 2$,relamp); text(v(lifres),6/6,temp);

text(v(lifres),4/6,vdrift);text(v(lifres),5/6,vdrift2);

$\operatorname{standardeviation}=\operatorname{std}(g \operatorname{sn}(\mathrm{A}, \mathrm{vd}+\operatorname{sep} / 2, \mathrm{v}, \mathrm{T})+\mathrm{gsn}(\mathrm{A}, \mathrm{vd}-\operatorname{sep} / 2, \mathrm{v}, \mathrm{T})+\mathrm{gsn}(\mathrm{A} 2, \mathrm{vd}+\operatorname{sep} / 4, \mathrm{v}, \mathrm{T})+\operatorname{gsn}(\mathrm{A} 2, \mathrm{vd}-\operatorname{sep} / 4, \mathrm{v}, \mathrm{T})-$ lifd) 


\section{Appendix 4}

$\%$ Sinefit $\%$

\% Created by E. Reynolds (June 2001); modified by C. Teodorescu (September 2001)

\%This MATLAB program plots the relative phase difference between the signals received

\%by the two probe tips of the two-tip probe array as a function of probe orientation

$\%$ with respect to the magnetic field.

$\%$ This function requires that the file have the raw time series from a double probe,

$\%$ the probe orientation angles in vector form,

$\%$ the \# of realizations

$\%$ and the sub-array (range) to be processed

\%It then determines the best-fit sinusoidal curve and the parameters: wavevector

\%parallel component, wavevector perpendicular component,wave propagation angle and wavelength

function [angles, $\mathrm{x} 1, \mathrm{y} 1, \mathrm{dx} 0]=$ sinefit(file,angles,reals,range)

close all;

samples $=4096 ; \%$ number of data points in the array

probesep $=0.35 ; \%$ probe tip seperation in $\mathrm{cm}$

$\mathrm{l}=$ size(angles); $\% 1=$ number of probe angles

fid=fopen(file);

signal=fscanf(fid, '\%f',[samples, $40 *$ reals]);fclose(fid);\%read data

power=abs(fft(signal(:,1:2:40*reals)). ${ }^{*} \operatorname{conj}(f f t(\operatorname{signal}(:, 2: 2: 40 *$ reals $\left.)))\right) ; \%$ calculate the cross-correlated

power and phase

phase $=$ angle $\left(\mathrm{fft}(\operatorname{signal}(:, 1: 2: 40 *\right.$ reals $)) \cdot{ }^{*} \operatorname{conj}(\mathrm{fft}(\operatorname{signal}(:, 2: 2: 40 *$ reals $\left.)))\right)$;

for $\mathrm{i}=1: 20 ; \quad \%$ all measurements

for $\mathrm{j}=1$ :reals;

$\mathrm{x} 0(\mathrm{i}, \mathrm{j})=\operatorname{sum}($ power(range, $i * 3-3+\mathrm{j}) .{ }^{*}$ phase $\left(\right.$ range, $\left.\left.i^{*} 3-3+\mathrm{j}\right)\right) / \operatorname{sum}($ power(range, $\left.\mathrm{i} * 3-3+\mathrm{j})\right) ; \%$ weighted average phase

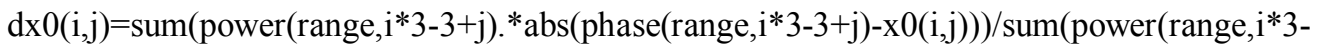

$3+\mathrm{j})$ ); \%weighted standard deviation

end

end

for $\mathrm{i}=1: 20$;

$x(i)=\operatorname{sum}(x 0(i,:) . / d x 0(i,:)) / \operatorname{sum}(1 . / d x 0(i,:)) ;$ 


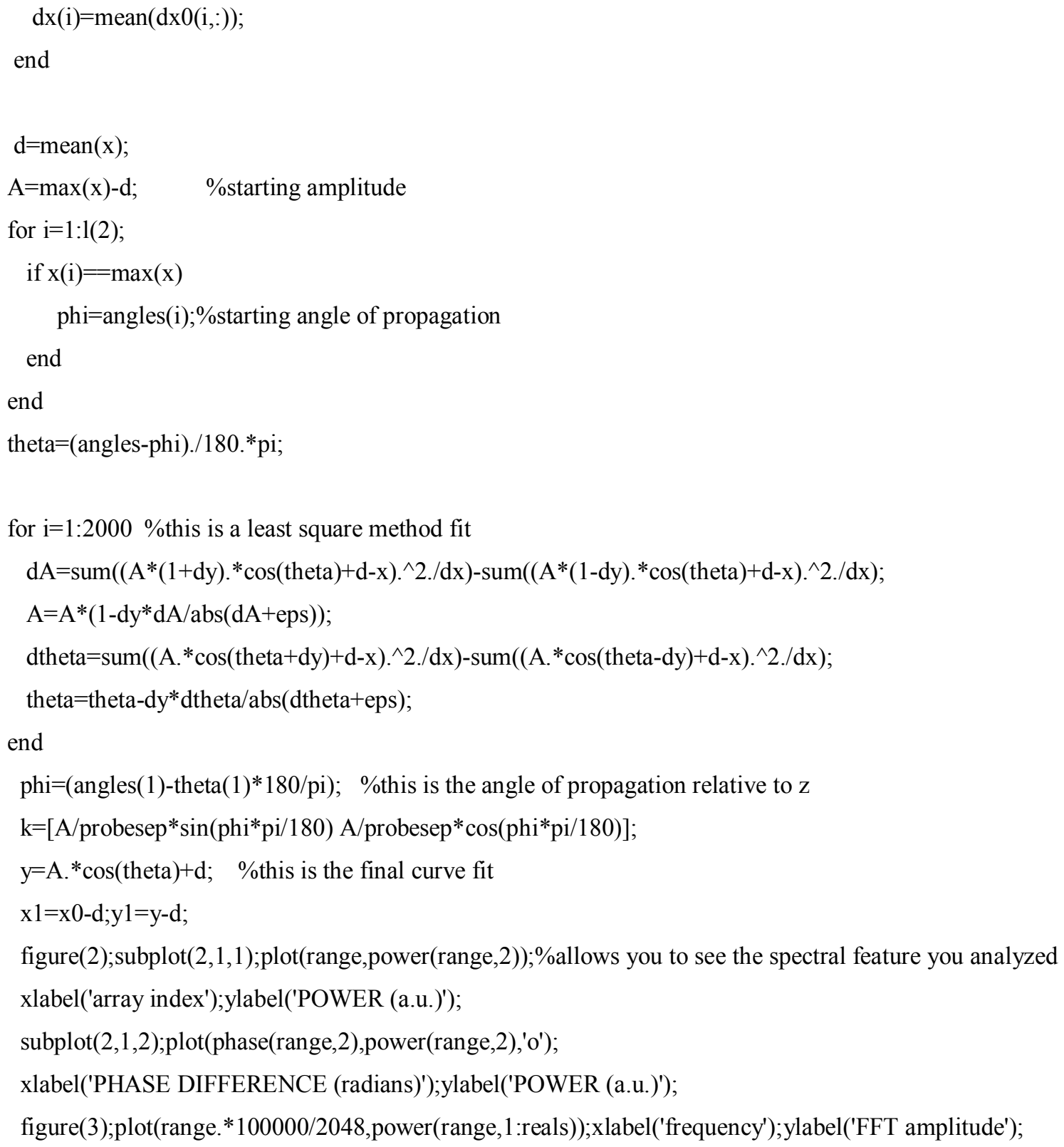

aaa=power(range, 1)+power(range, 2)+ power(range,3)+ power(range,4)+power(range,5)+power(range,6)+ power( range,7)+ power(range, 8)+ power(range,9)+ power(range,10)+power(range,11)+power(range, 12)+power(range,1 $3)+$ power(range, 14)+power(range,15)+power(range,16); \%this calculates the power of the first 16 realizations (used for smoothing the spectra)

figure(4);semilogy(range.*100000/(2048*1.1e4*2.5),aaa/16);xlabel('Normalized frequency');ylabel('FFT amplitude'); \%this is a semi-log scale plot of the spectra figure(1) plot(angles,y-d,'k');hold;\%and you can see it plotted against the data here 


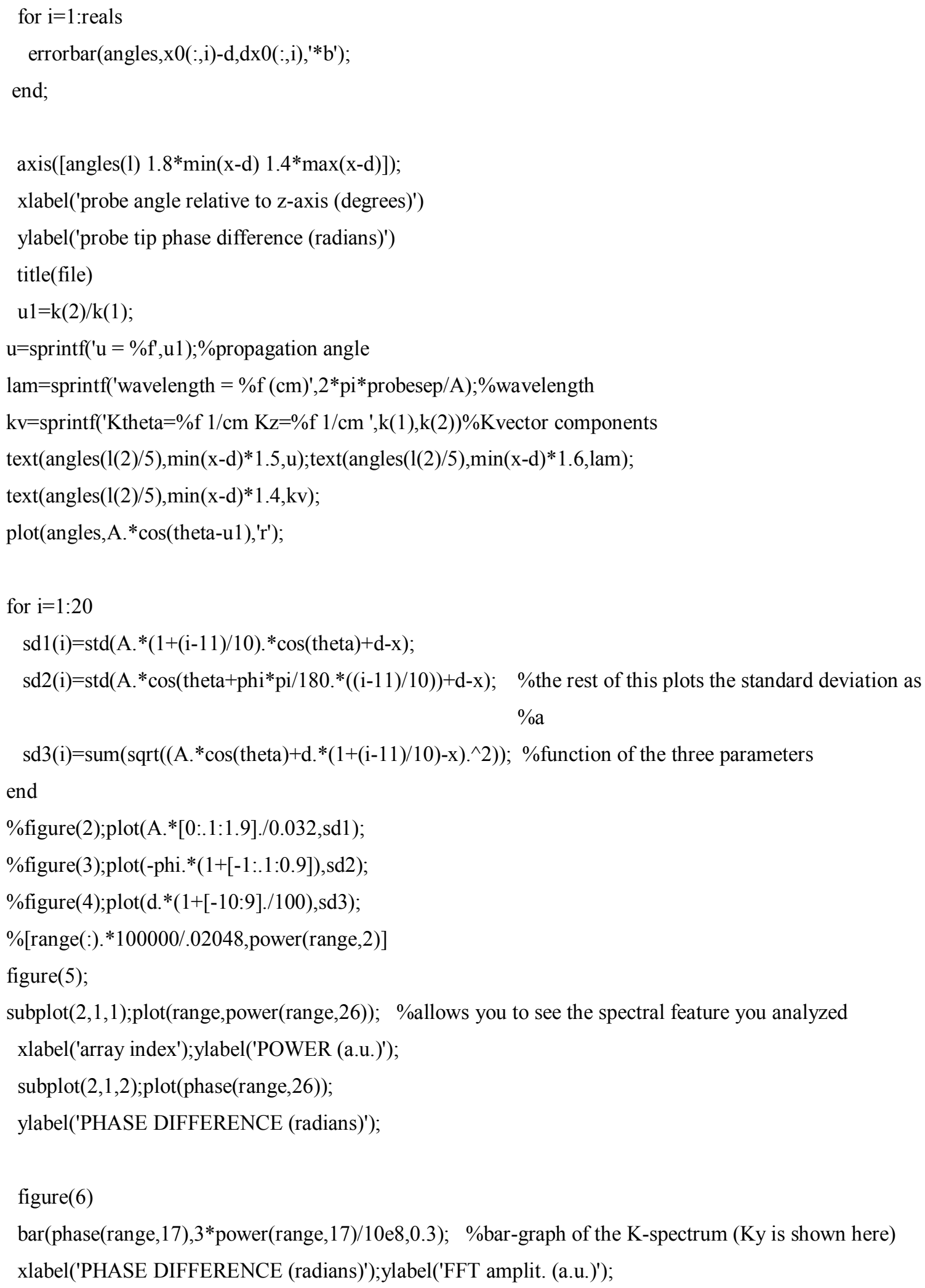




\section{Appendix 5}

\section{Derivation of the shear-modified ion-cyclotron waves dispersion relation}

Using the definitions of (50), the real part of the SMIC wave general dispersion relation (44) can be written as

$$
\begin{aligned}
& \sum_{n} \Gamma_{n}(b)\left\{\xi_{0 i}\left(-\frac{1}{\xi_{n i}}-\frac{1}{2 \xi_{n i}{ }^{3}}\right)-\frac{1}{u \omega_{c i}} \frac{d v_{d i}}{d x}\left[1+\xi_{n i}\left(-\frac{1}{\xi_{n i}}-\frac{1}{2 \xi_{n i}{ }^{3}}\right)\right]\right\}+ \\
& +\left(1-2 \xi_{n e}^{2}\right)\left(\frac{T_{i}}{T_{e}}+\frac{1}{u \omega_{c e}} \frac{d v_{d e}}{d x}\right)+k^{2} \lambda_{D}^{2}+1=0 .
\end{aligned}
$$

In the limits of (50) and $\frac{d v_{d e} / d x}{u \omega_{c e}}<<1$, with $\left(k \lambda_{\mathrm{D}}\right)^{2}<<1$, neglecting the terms of the order of $\zeta_{n i}{ }^{-3}$ and higher and the terms of the order of $\zeta_{n e}{ }^{2}$ and higher, (A.5.1) becomes

$$
\begin{aligned}
& \sum_{n} \Gamma_{n}(b)\left[\frac{\omega_{1 r}}{\omega_{1 r}-n \omega_{c i}}-\frac{\omega_{1 r}}{\omega_{1 r}-n \omega_{c i}}\left(\frac{k_{z} v_{t i}}{\omega_{1 r}-n \omega_{c i}}\right)^{2}+\frac{1}{u \omega_{c i}} \frac{d v_{d i}}{d x}\left(\frac{k_{z} v_{t i}}{\omega_{1 r}-n \omega_{c i}}\right)^{2}\right]+ \\
& +\frac{T_{i}}{T_{e}}+1=0 .
\end{aligned}
$$

This can be re-arranged as

$$
\sum_{n} \Gamma_{n}(b) \frac{\omega_{1 r}}{\omega_{1 r}-n \omega_{c i}}\left[-1-\left(\frac{k_{z} v_{t i}}{\omega_{1 r}-n \omega_{c i}}\right)^{2}\left[1-\frac{1}{u \omega_{c i}} \frac{d v_{d i}}{d x}\left(1-\frac{n \omega_{c i}}{\omega_{1 r}}\right)\right]\right]+\frac{T_{i}}{T_{e}}+1=0 .
$$

For each $n$ term, we have

$$
1-\Gamma_{n}(b) \frac{\omega_{1 r}}{\omega_{1 r}-n \omega_{c i}}-\Gamma_{n}(b) \frac{\omega_{1 r}}{\omega_{1 r}-n \omega_{c i}} \sigma_{n}^{2}+\frac{T_{i}}{T_{e}}=0
$$

In plasmas described in this dissertation, the first two terms of (A.5.4) are larger than the last two terms. Therefore, (A.5.4) can be separated into two parts, as follows

$$
\begin{aligned}
& 1-\Gamma_{n}(b) \frac{\omega_{1 r}}{\omega_{1 r}-n \omega_{c i}}=0 . \\
& \Gamma_{n}(b) \frac{\omega_{1 r}}{\omega_{1 r}-n \omega_{c i}} \sigma_{n}^{2}-\frac{T_{i}}{T_{e}}=0 .
\end{aligned}
$$


The solution of (A.5.5) is $\omega_{1 r}=\frac{n \omega_{c i}}{1-\Gamma_{n}(b)} \geq n \omega_{c i}$. The solution is similar to that of the simplified dispersion relation of CDEIC in homogeneous-ion-flow plasmas [Kindel and Kennel, 1971]. The effect of parallel-velocity shear on the SMIC wave dispersion relation comes as a small correction to this general solution. (A.5.6) can be re-arranged as

$$
\Gamma_{n}(b) \frac{\omega_{1 r}}{\omega_{1 r}-n \omega_{c i}} \sigma_{n}^{2}=\frac{\left(\omega_{1 r}-n \omega_{c i}\right)^{2}}{k_{z}^{2}} \frac{T_{i}}{T_{e}} \frac{m_{i}}{k_{B} T_{i}} \equiv \frac{\left(\omega_{1 r}-n \omega_{c i}\right)^{2}}{k_{z}^{2} c_{s}^{2}} .
$$

Further more, using $\omega_{\mathrm{lr}}=n \omega_{\mathrm{ci}}+\varepsilon$, where $n \omega_{\mathrm{ii}}>>\mathcal{\varepsilon}$,

$$
\Gamma_{n}(b) k_{z}^{2} c_{s}^{2} \sigma_{n}^{2}=\frac{\left(\omega_{1 r}-n \omega_{c i}\right)^{3}}{\omega_{1 r}} \equiv \frac{\varepsilon^{3}}{n \omega_{c i}+\varepsilon} .
$$

The expression above becomes

$$
\Gamma_{n}(b) k_{z}^{2} c_{s}^{2} \sigma_{n}^{2} n \omega_{c i} \approx \varepsilon^{3},
$$

Thus, the SMIC wave simplified dispersion relation (55) is obtained,

$$
\omega_{1 r} \approx n \omega_{c i}+\left[n \omega_{c i} \Gamma_{n}(b) k_{z}^{2} c_{s}^{2} \sigma_{n}^{2}\right]^{1 / 3} .
$$




\section{Appendix 6. On the analysis of SMIA wave growth rate}

The peaks of the oscillation amplitude shown in Fig. A.6.1, which is also Fig. 61(b), can be divided into several groups according to their frequency. These frequencies are between 0.1 and $1.6 \mathrm{kHz}$ and are the frequencies of the features seen in the FFT of the timeseries within $\Delta t=7 \mathrm{~ms}$ after the wave onset (see Fig. A.6.2). This suggests that there are several narrowband modes, well separated in frequency, destabilized in the same time at the onset of the instability. Three modes with average frequencies equal to 0.7 (circles), 1.2 (squares) and 1.5 (triangles) $\mathrm{kHz}$ can be seen. In Fig. 61b, the $0.7 \mathrm{kHz}$-frequency mode (circles) increases its amplitude within the first $3.5 \mathrm{~ms}$ from the wave onset. After that time the peaks can no longer be detected, signifying that this mode has vanished. The other modes coexist for almost the entire duration of the wave growth, but eventually only one mode dominates, as seen in the FFT of the timeseries well after the wave onset. For three modes that can be detected during the wave growth the least-square method of fitting, an exponential function to data points for determining the growth rate can be applied. The mode with the frequency $f_{r}=1.0 \mathrm{kHz}$ eventually dominates, leading to the existence of a SMIA mode with ion-frame frequency $f_{1 \mathrm{r}}=1.2 \mathrm{kHz}$. This value is confirmed by the FFT analysis of the same time duration $(\Delta t=7 \mathrm{~ms})$ of the timeseries well after $(t=33 \mathrm{~ms})$ the wave onset at $t=11.3 \mathrm{~ms}$. From $t=33 \mathrm{~ms}$ to $t=40 \mathrm{~ms}$ only one, narrower, spectrum feature is detected at $1 \mathrm{kHz}$, as shown in Fig. A.6.2 where both graphs have the same normalization constant.

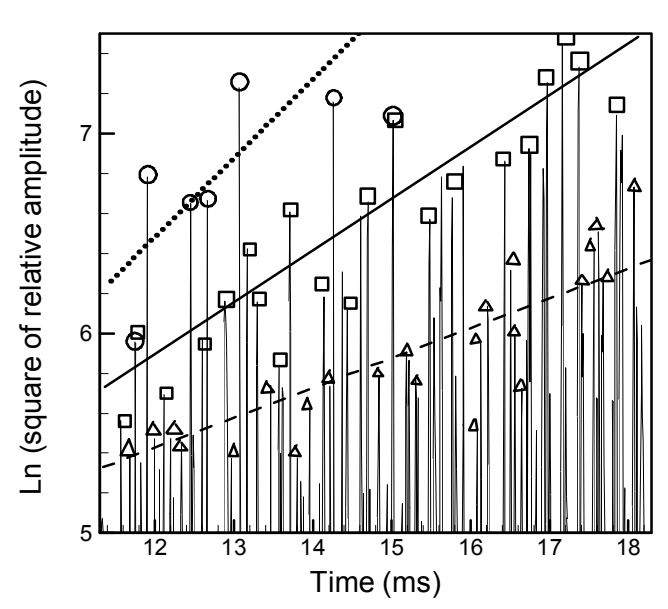

Fig. A.6.1.

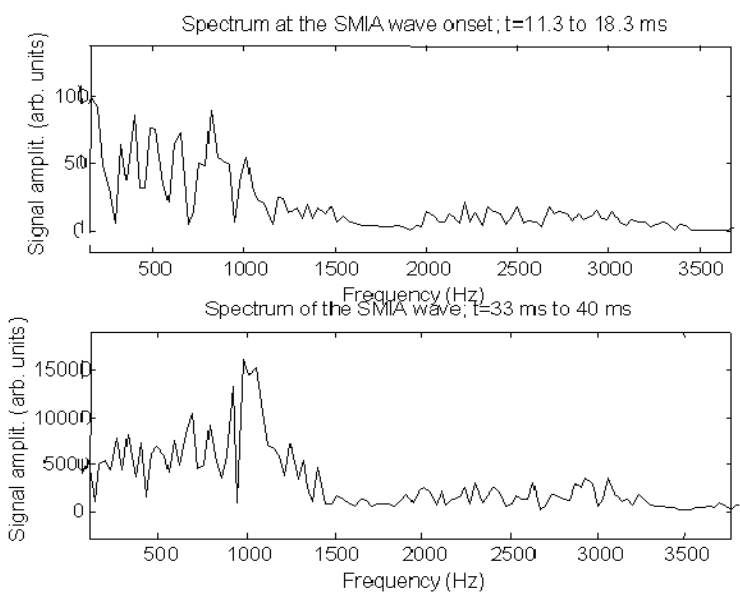

Fig. A.6.2.

The largest contribution to the uncertainty in determining the wave growth rate comes from the position in the timeseries of the amplitude peaks (separated by a time corresponding 
to a given frequency, in this case either $0.7,1.2$ or $15 \mathrm{kHz}$ ) used for fitting a straight line through them. All peaks are counted, irrespective of their heights. When the peaks are extremely close, the peak with the largest height is counted. Because not all peaks are exactly separated by the same period $\tau_{\mathrm{p}}=1 / f$, some of them will correspond to a non-cyclic phase difference $\Delta \Phi(t)$. If the distribution of the peaks is a Gaussian, the uncertainty in determining the rate is at its minimum because most of the peaks are separated by a wave period $\tau_{\mathrm{p}}$. If the occurence of the peaks is random, the distribution would be independent of $\Phi$ and the peaks indicate bursts rather than an approximately sinusoidal instability associated with a wave. Fig. A.6.3 shows this Gaussian distribution for the mode with frequency $f_{l r}=1.2 \mathrm{kHz}$ (squares), whereas the other two mode peaks are distributed rather randomly with phase differences $\Delta \Phi$ between $-\pi$ and $\pi$. This indication suggests that the mode with the frequency $1.0 \mathrm{kHz}$ is the wave feature whose growth rate is indicative of the SMIA wave growth rate.

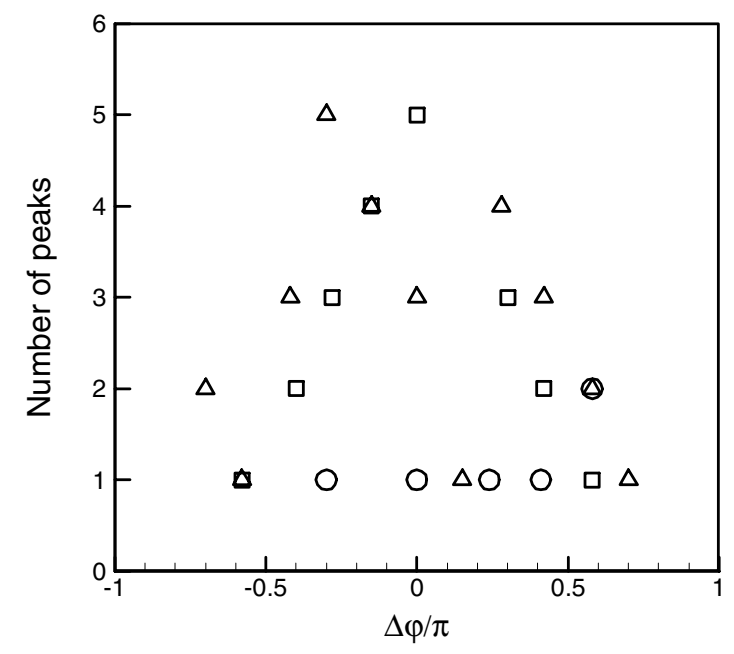

Fig. A.6.3.

The concept that several modes are being destabilized at the SMIA wave onset can be illustrated using a simple model. Here, three oscillations of different initial amplitude (2.2, 3.1 and 4 arbitrary units, respectively) and different growth rates $(0.06,0.04$ and 0.025 arbitrary units, respectively) are considered. Their frequencies are such that the frequency of the second and third oscillations are approximately harmonics of the first oscillation frequency: $f_{1}=3$ arb. units, $f_{2}=5.2 \mathrm{arb}$. units and $f_{3}=7.5 \mathrm{arb}$. units. Fig. A.6.4 shows half of the timeseries obtained by summing the three oscillations. The observed resemblance with Fig. 
$61 \mathrm{~b}$ lends merit to the idea that there could be several modes destabilized in the experimental study of the SMIA wave onset.

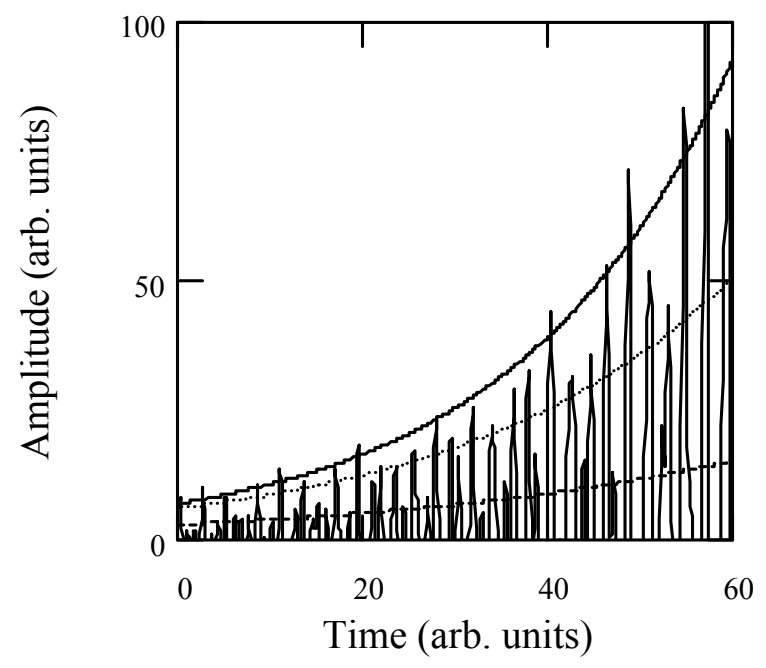

Fig. A.6.4. Summed amplitude of the three oscillations. The equations of the three fitted exponentials are $2.2 e^{0.043(t+27)}$ (solid line), $3.1 e^{0.035(t+20)}$ (dotted line) and $4 e^{0.027(t-10)}$ (dashed line), respectively.

The resemblance is enhanced if, as showed in Fig. A.6.5, the absolute value of the sum of the amplitudes is considered. Recall that Fig. $61 \mathrm{~b}$ was obtained by subtracting the measured timeseries from a fitted line (see Fig. 61a) to remove the initial pulse.

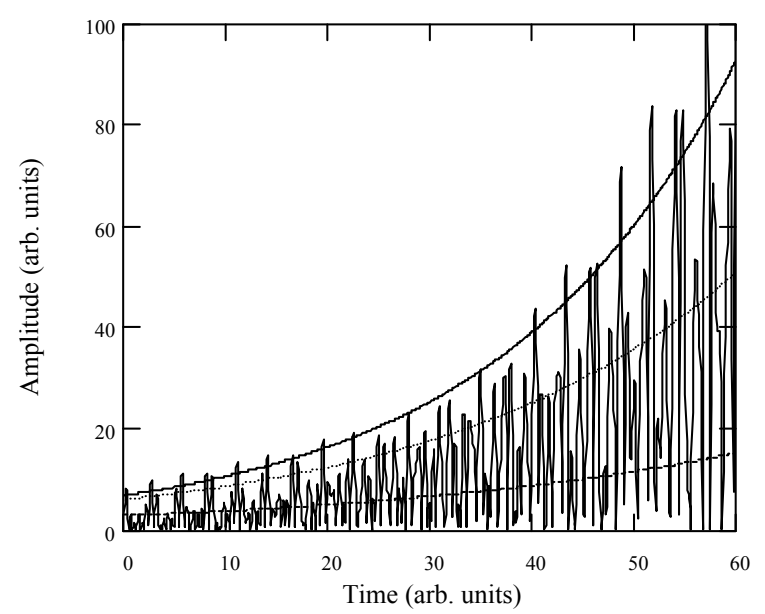

Fig. A.6.5.Absolute value of the summed amplitude of the oscillations. 
The growth rate analysis of this model is performed in the same manner as for the SMIA wave growth rate. Three sets of peaks of the oscillation are identified based on their period and growing amplitude. Then, an exponential is fitted through the peaks of each of the three sets, as shown in Fig. A.6.4. Each fitted exponential has a growth rate not more than $25 \%$ larger or smaller than the actual growth rate of each of the three individual oscillations. The effect of the fitting parameter, i.e., the exponential constant, is shown in Fig. A.6.6.

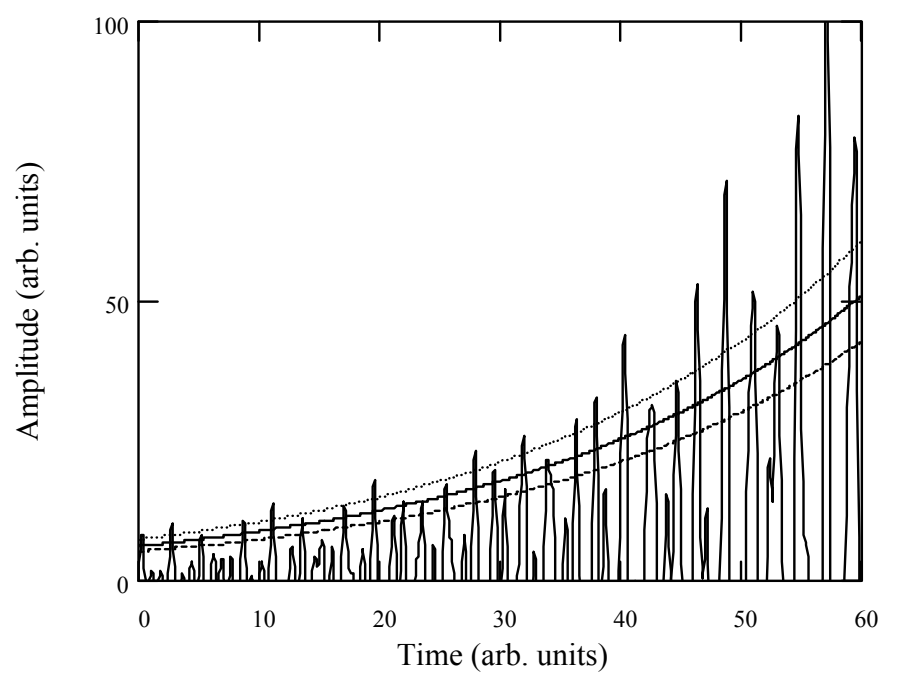

Fig. A.6.6. Effect of varying the fitting parameter of the fitted exponential through the amplitude peaks of the second mode (growth rate 0.04 a. u.). Fitted growth rate is $0.035 \mathrm{a}$. $\mathrm{u}$. and the fitting parameter is $+25 \mathrm{a}$. u. (dotted line), +20 a. u. (solid line) and +15 a. u. (dashed line).

The effect of varying this constant in fitting the exponential is smaller than the effect of varying the fitted growth rate, which is shown in Fig. A.6.7, for each of the three oscillations. As seen by comparing Fig. A.6.6. and Fig. A.6.7, a change by $10 \%$ in the value of the growth rate is larger than a change by $25 \%$ in the exponential constant.

It can be thus inferred that a simple model in which three individual oscillations with distinct growth rates, initial amplitudes, and frequencies are summed can be analyzed by the algorithm applied to the analysis of the SMIA wave growth. Based on the growth rate analysis performed on the model, it can be inferred that the fitted growth rate could be off the actual wave growth rate by as much as $40 \%$. This $40 \%$ value of the error is larger than the $32 \%$ error indicated in the graphs of Fig. 60, showing that the errors in the experimental determinations of the growth rates are actually smaller than the errors obtained using this simple model. 


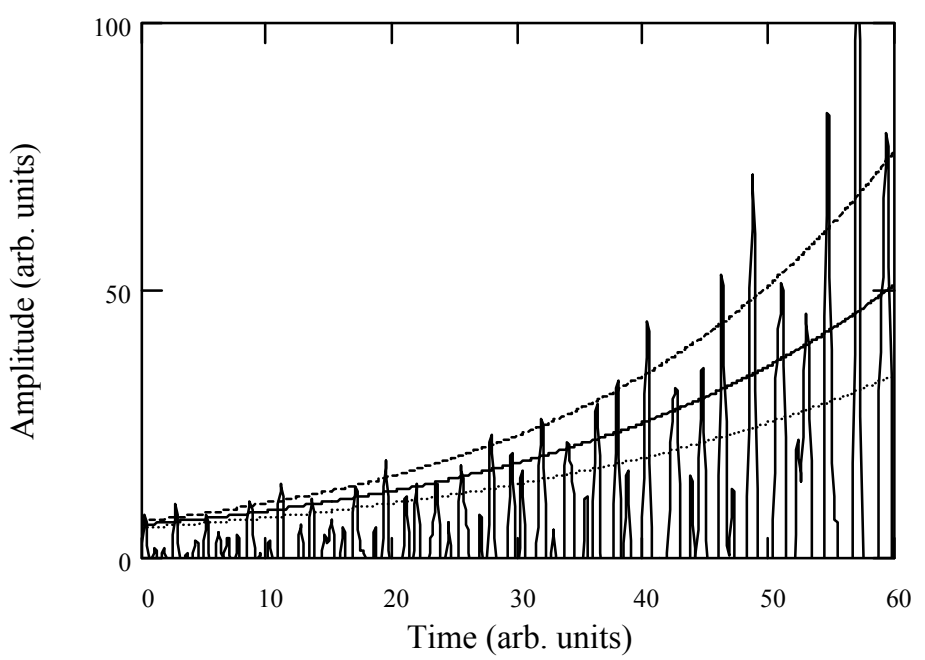

Fig. A.6.6. Effect of varying the growth rate of the fitted exponential through the amplitude peaks of the second mode (growth rate 0.04 a. u.). Fitted growth rate is 0.03 a. u. (dotted line), 0.035 a. u. (solid line) and 0.04 a. u. (dashed line). Fitting parameter is $+20 \mathrm{a}$. $\mathrm{u}$. 


\section{Catalin Teodorescu}

\section{Education:}

1998-2002 West Virginia University, Morgantown, WV, USA

Ph.D. in Physics.

Dissertation: Laboratory Investigation of Electrostatic Ion Waves Modified by Parallel-Ion-Velocity Shear.

1989-1994 “Al. I. Cuza” University, Iasi, Romania

B.Sc. in Physics.

Experience:

1999-2002 West Virginia University, Morgantown, WV, USA

- Research Assistant

1998-2000 West Virginia University, Morgantown, WV, USA

- Teaching Assistant

1994-1997 “Nicolina” Technical Senior High-School, Iasi, Romania

Honors and Awards:

1999 Outstanding Physics Teaching Assistant, West Virginia University.

2002 The William E. Vehse Travel Award for Doctoral Students, West Virginia University.

2002 Invited oral presentation at the $44^{\text {th }}$ Annual Meeting of the American Physical Society Division of Plasma Physics, Orlando, FL.

\section{Publications:}

M. E. Koepke, C. Teodorescu, E. W. Reynolds, C. C. Chaston, C. W. Carlson, J. P. McFadden and R. E. Ergun, Inverse Ion Cyclotron Damping: Laboratory Demonstration and Space Ramifications, Phys. Plasmas, in press, 2003.

M. E. Koepke, C. Teodorescu and E. W. Reynolds, Space-relevant laboratory studies of ion-acoustic and ion-cyclotron waves driven by parallel-velocity 
shear, Plasma Phys. Contr. Fusion, in press, 2003.

E. E. Scime, A. M. Keesse, R. S. Spangler, M. E. Koepke, C. Teodorescu and E. W. Reynolds, Evidence for thermal anisotropy effects on shear modified ion acoustic instabilities, Phys. Plasmas, 9, 4399, 2002.

C. Teodorescu, M. E. Koepke and E. W. Reynolds, On the Role of Ion Temperature Anisotropy in the Growth and Propagation of Shear-Modified Ion-Acoustic Waves, J. Geophys. Res., 108 (A1), 2049, 2003.

C. Teodorescu, E. W. Reynolds and M. E. Koepke, Observation of Inverse IonCyclotron Damping Induced by Parallel-Velocity Shear, Phys. Rev. Lett., 89, 105001, 2002.

C. Teodorescu, E. W. Reynolds and M. E. Koepke, Experimental Verification of the Shear-Modified Ion-Acoustic Instability, Phys. Rev. Lett., 88, 185003, 2002.

M. E. Koepke, M. W. Zintl, C. Teodorescu, E. W. Reynolds, G. Wang and T. N. Good, Inhomogeneous Magnetic-Field-Aligned Ion Flow Measured in a $\mathbf{Q}$ machine, Phys. Plasmas, 9, 3225, 2002.

\section{Other publications:}

C. Teodorescu, Observation of inverse ion cyclotron damping induced by inhomogeneous magnetic-field-aligned ion flow, invited oral presentation at the $44^{\text {th }}$ Meeting of the American Physical Society Division of Plasma Physics, November 2002, Orlando, FL (to be presented).

C. Teodorescu, E.W. Reynolds and M. E. Koepke, Observation of multi-harmonic ion cyclotron waves in the presence of inhomogeneous ion parallel flow, invited oral presentation at the 2002 International Union of Radio Science General Assembly Meeting, August 2002, Maastricht, the Netherlands.

C. Teodorescu, E.W. Reynolds and M.E. Koepke, On the role of ion temperature anisotropy in the growth and propagation of shear modified ion acoustic waves, poster at the 2002 Spring American Geophysical Union Meeting, May 2002, Washington, D.C.

C. Teodorescu, E.W. Reynolds and M. E. Koepke, Experimental verification of the shear modified ion acoustic instability, poster at the 2001 Fall A. G. U. Meeting, San Francisco, CA.

E. Sarid, C. Teodorescu, P. Marcus and J. Fajans, Breaking the Azimuthal Symmetry - Jumping off-axis or Staying Away from the Axis?, Proceedings of the 2001 Non-Neutral Plasma Conference, San Diego, CA, July 2001. 
C. Teodorescu and M. E. Koepke, Combination of Autoresonance and PeriodicPulling in a Nonlinear Oscillator, poster at the $42^{\text {nd }}$ American Physical Society Division of Plasma Physics Meeting, Quebec, Canada, October 2000.

\section{Other professional activity:}

Referee for Journal of Geophysical Research and Physics of Plasmas.

\section{Memberships:}

American Physical Society

American Geophysical Union 